Extensão de GENSMAC para escoamentos de fluidos governados pelos modelos integrais Maxwell e K-BKZ

Manoel Silvino Batalha de Araújo 


\title{
Extensão de GENSMAC para escoamentos de fluidos governados pelos modelos integrais Maxwell e K-BKZ
}

\author{
Manoel Silvino Batalha de Araújo
}

Orientador: Prof. Dr. Murilo Francisco Tomé

Tese apresentada ao Instituto de Ciências Matemáticas e de Computação - ICMC-USP, como parte dos requisitos para obtenção do título de Doutor em Ciências Ciências de Computação e Matemática Computacional.

\author{
USP - São Carlos
}

Maio/2006 
Aos meus pais Ana Beth P. Batalha e Manoel Raimundo L. de Araújo, à minha esposa Eliza e ao meu filho 



\section{Agradecimentos}

Gostaria de agradecer a todos que me ajudaram, ao longo da minha vida, para que eu chegasse até este momento. Primeiramente a Deus. Aos meus pais, Raimundo e Beth, que desde sempre nunca mediram esforços para que eu sempre continuasse a estudar, muitas vezes abdicando de coisas materiais e trabalhando dobrado na feira do Ver-O-Peso para adquirir livros e materiais escolares. Muito obrigado a vocês dois. Espero ter feito com que o esforço de vocês tenha valido a pena. Agradeço também aos meus irmãos Cleide, Rosana e Wallace pela compreensão e apoio que sempre me deram.

Obrigado a minha esposa Eliza, que sempre me apoiou, em todos o momentos, e nunca esteve ausente quanto precisei. Obrigado pela sua paciência e pelo seu amor. Esse doutorado também é seu.

Também não posso deixar de agradecer aos responsáveis pela minha formação. Meus mestres. Infelizmente não é possível citar todos aqui, mas gostaria de expressar minha gratidão a todos, desde minhas professoras e professores do primeiro grau, na escola "Externato Batista"e na escola "Madre Zarife Sales", no bairro do Guamá, em Belém, até os professores que tive na Escola Técnica Federal do Pará e na Universidade Federal do Pará.

Obrigado também aos meus professores de pós-graduação do Instituto de Ciências Matemáticas e de Computação da Universidade de São Paulo. Em especial ao professor Murilo Francisco Tomé, meu orientador e amigo, que me deu todas as condições para prosseguir na nossa área de pesquisa, e ao professor José Alberto Cuminato, o Poti, que me recebeu muito bem ao chegar no Instituto e que me acompanhou por dois anos do meu doutorado.

Agradeço também aos amigos que estiveram comigo nesses quatro anos. Obrigado ao Maurício, ao Zé Paulo, ao Marcelinho e aos demais amigos da "fase análise funcional". Aos colegas do LCAD Andréa, Cássio, Dayene e Gilcilene, que também estiveram presentes 
neste tempo e a todos o demais colegas e amigos que fiz ao longo desses quatro anos.

Finalmente agradeço à Universidade Federal do Pará, na qual sou professor do departamento de Matemática, por ter concedido meu afastamento para fazer meu doutoramento, e à CAPES pelo apoio financeiro. 


\section{Resumo}

Este trabalho tem como objetivo desenvolver um método numérico para simular escoamentos incompressíveis, isotérmicos, confinados ou com superfícies livres, de fluidos viscoelásticos governados pelos modelos integrais de Maxwell e K-BKZ (Kaye-Bernstein, Kearsley e Zapas). A técnica numérica apresentada é uma extensão do método GENSMAC (Tomé e McKee - J. Comp. Phys., (110), pp 171-186, 1994) para a solução das equações de conservação, juntamente com as equações constitutivas integrais de Maxwell e K-BKZ. As equações governantes são resolvidas pelo método de diferenças finitas em uma malha deslocada. O tensor de Finger, $\mathbf{B}_{t^{\prime}}(t)$, é calculado com base nas idéias do método de campos de deformação (Peters et al. - J. Non-Newtonian Fluid Mech. (89), pp 209-228, 2000), de maneira que não há a necessidade de seguir a trajetória da partícula de fluido para descrever a história de deformação da partícula. Uma abordagem diferente para a discretização do instante passado é utilizada e o tensor de Finger e o tensor das tensões são calculados utilizando um método de segunda ordem. A validação do método numérico descrito nesse trabalho foi feita utilizando o escoamento em um canal bidimensional e a solução numérica obtida para a velocidade e para as componentes de tensão com o modelo de Maxwell foram comparadas com as respectivas soluções analíticas no estado estacionário, mostrando excelente concordância. Os resultados numéricos para a simulação do escoamento em uma contração planar 4 : 1 mostraram bons resultados, tanto qualitativos quanto quantitativos, quando comparados com os resultados experimentais de Quinzani et al. ( J. Non-Newtonian Fluid Mech. (52), pp 1-36, 1994 ). Além disso, utilizando o modelo de Maxwel e K-BKZ, o escoamento em uma contração planar $4: 1$ foi simulado para vários números de Weissenberg e os resultados obtidos estão de acordo os encontrados na literatura. Resultados numéricos de escoamentos com superfícies livres modelados pelas equações integrais de Maxwell e K-BKZ são apresentados. Em particular, a simulação numérica do jato oscilante para diferentes números de Weissenberg e diferentes números de Reynolds é apresentada.

Palavras chave: Escoamentos incompressíveis, modelo Maxwell, modelo K-BKZ, diferenças finitas, contração planar, superfície livre, reologia computacional 


\section{Abstract}

The aim of this work is to develop a numerical technique for simulating incompressible, isothermal, free surface (also confined) viscoelastic flows of fluids governed by the integral models of Maxwell and K-BKZ (Kaye-Bernstein, Kearsley and Zapas). The numerical technique described herein is an extension of the GENSMAC method (Tome and McKee, J. Comput. Phys., 110, pp. 171-186, 1994) to the solution of the momentuum and mass conservation equations together with the integral constitutive Maxwell and KBKZ equations. The governing equations are solved by the finite difference method on a staggered grid using a Marker-and-Cell approach. The fluid is represented by marker particles on the fluid surface only. This provides the visualization and location of the fluid free surface so that the free surface stress conditions can be applied. The Finger tensor $\mathbf{B}_{t^{\prime}}(t)$ is computed using the ideias of the deformation fields method (Peters et al. J. Non-Newtonian Fluid Mech., 89, pp. 209-228, 2001) so that it is not necessary to track a fluid particle in order to calculate its deformation history. However, in this work modifications to the deformation fields method are introduced: the past time is discretized using a different formula, the Finger tensor $\mathbf{B}_{t^{\prime}}(\mathbf{x}, t)$ is obtained by a second order method and the stress tensor $\tau(\mathbf{x}, t)$ is computed by a second order quadrature formula. The numerical method presented in this work is validated by simulating the flow of a Maxwell fluid in a two-dimensional channel and the numerical solutions of the velocity and the stress components are compared with the respective analytic solutions providing a good agreement. Further, the flow through a 4:1 planar contraction of a specific fluid studied experimentally by Quinzani et al. (J. Non-Newtonian Fluid Mech., $\mathbf{5 2}$, pp. 1-36, 1994) was simulated and the numerical results were compared qualitatively and quantitatively with the experimental results and very good agreement was obtained. The Maxwell and the K-BKZ models were applied to simulate the 4:1 planar contraction problem using various Weissenberg numbers and the numerical results were in agreement with those published in the literature. Finally, numerical results of free surface flows using the Maxwell and K-BKZ integral constitutive equations are presented. In particular, the numerical simulation of jet buckling using several Weissenberg numbers and various Reynolds numbers are presented.

Keywords: Incompressible flows, Maxwell model, K-BKZ model, finite difference, planar contraction, free surface, computational rheology. 


\section{Sumário}

Introdução 11

1 Conceitos Gerais 5

1.1 Desenvolvimentos de GENSMAC . . . . . . . . . . . . . . . . . . . . 5

1.2 Alguns conceitos em reologia . . . . . . . . . . . . . . . . . 8

1.3 Alguns modelos constitutivos integrais: Maxwell e K-BKZ . . . . . . . . . 12

2 Solução Numérica dos modelos integrais de Maxwell e K-BKZ 15

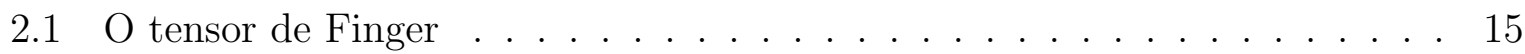

2.2 Forma original do método de campos de deformação . . . . . . . . . . . . . 18

2.3 Cálculo do tensor das tensões $\tau(t) \ldots \ldots \ldots$. . . . . . . . . . 22

2.3 .1 Discretização do tempo passado $t^{\prime} \ldots \ldots$. . . . . . . . . . . . 23

2.3 .2 Convecção dos tensores de Finger . . . . . . . . . . . . . . . . 25

2.3 .3 Cálculo das tensões . . . . . . . . . . . . . . . . . . . . 26

2.4 Validação do cálculo das tensões . . . . . . . . . . . . . . . . . . . . . . . 28

3 Extensão de GENSMAC para modelos integrais de Maxwell e K-BKZ 35

3.1 Idéia Básica do método GENSMAC . . . . . . . . . . . . . . . . . . . . . . 35

3.2 Equações governantes . . . . . . . . . . . . . . . . . . . . . 36

3.3 Condições de contorno . . . . . . . . . . . . . . . . . . . . . . . . 38

3.4 Cálculo do tensor de Finger nos contornos . . . . . . . . . . . . . . . . . . 39

3.4.1 Cálculo do tensor de Finger nos contornos rígidos . . . . . . . . . . 39

3.4 .2 Cálculo do tensor de Finger nos injetores . . . . . . . . . . . . . . . 39

3.4.3 Cálculo do tensor de Finger nos ejetores . . . . . . . . . . . . . . . 40

3.5 Condições de superfície livre . . . . . . . . . . . . . . . . . . . 40

3.6 Método de Solução . . . . . . . . . . . . . . . . . . . . . . 41 
3.7 Aproximação pelo método de diferenças finitas . . . . . . . . . . . . . . . 42

3.7.1 Aproximação do tensor de Finger . . . . . . . . . . . . . . . . . . . 45

3.7.2 Aproximação do tensor de Finger nos contornos da malha . . . . . 46

3.7 .3 Aproximação dos termos convectivos . . . . . . . . . . . . . . 52

3.7 .4 Aproximação das condições de tensão na superfície livre . . . . . . . 53

3.7.5 Cálculo do passo de tempo . . . . . . . . . . . . . 56

4 Resultados Numéricos $\quad 57$

4.1 Validação do Método Numérico ． . . . . . . . . . . . . . . . . 57

4.1 .1 Modelo Maxwell . . . . . . . . . . . . . . . . . . . . . . . 58

4.1 .2 Modelo K-BKZ . . . . . . . . . . . . . . . . . . . 60

4.1 .3 Comparação com resultados experimentais . . . . . . . . . . . . . 62

4.2 Simulação Numérica do Escoamento em uma Contração Planar 4:1 . . . 74

4.2 .1 Modelo Maxwell . . . . . . . . . . . . . . . . . . . . . . 80

4.2 .2 Modelo K-BKZ . . . . . . . . . . . . . . . . . . . . . . . . . . . 82

4.3 Simulação Numérica de Escoamentos com Superfícies Livres . . . . . . . . 88

4.3 .1 Simulação numérica do jato oscilante . . . . . . . . . . . . . . . . . 89

4.3 .2 Efeito da viscoelasticidade . . . . . . . . . . . . . . . . 91

4.3.3 Simulação do jato oscilante - modelo K-BKZ . . . . . . . . . . . . . 93 


\section{Introdução}

A simulação numérica de escoamentos de fluidos viscosos tem sido motivo de contínuas pesquisas em vários centros no mundo. No Brasil, esta área vem crescendo ao longo dos anos e já possui grupos em diversos centros de pesquisa. O grupo do LCAD (Laboratório de Computação de Alto Desempenho) do Instituto de Ciências Matemáticas e de Computação - ICMC da USP vem trabalhando há alguns anos nessa linha com vários projetos em andamento.

Pela sua importância em processos industriais, a simulação numérica de fluidos poliméricos vem ocupando um espaço cada vez maior. Some-se a isso o desenvolvimento de técnicas numéricas mais eficientes, computadores com maior capacidade de cálculos e, essencialmente, o desenvolvimento de equações constitutivas mais elaboradas, capazes de prever um número maior de efeitos viscoelásticos. Dentre essas equações, a equação K-BKZ [24, 7] é mencionada na literatura como uma das que melhor se ajusta aos resultados experimentais e por isso tem sido motivo de pesquisas recentes no que diz respeito a resultados numéricos [22, 39, 49]. Diferentemente de outras equações constitutivas, esta equação é definida exclusivamente na forma de uma equação integral, que armazena informações sobre a história da deformação do fluido em instantes anteriores. Essa peculiaridade traz desafios numéricos que estão sendo trabalhados ao longo dos anos por diversos grupos de pesquisa [22, 39, 54].

O método numérico apresentado nesta tese trata da simulação numérica de escoamentos de fluidos viscoelásticos governados pelos modelos constitutivos integrais K-BKZ

$$
\boldsymbol{\tau}(t)=\int_{-\infty}^{t} \sum_{m} \frac{a_{m}}{\lambda_{m}} e^{-\frac{t-t^{\prime}}{\lambda_{m}}} \frac{\alpha}{\alpha-3+\beta I_{1}+(1-\beta) I_{2}} \mathbf{B}_{t^{\prime}}(t) d t^{\prime}
$$

e o modelo convectivo superior de Maxwell (UCM - Upper-Convected Maxwell model), o 
qual chamaremos simplesmente modelo Maxwell, dado por

$$
\boldsymbol{\tau}(t)=\int_{-\infty}^{t} \frac{a}{\lambda} e^{-\frac{t-t^{\prime}}{\lambda}} \mathbf{B}_{t^{\prime}}(t) d t^{\prime}
$$

Nessas equações, os termos $a, \lambda, a_{m}, \lambda_{m}, \alpha$ e $\beta$ são parâmetros que dependem do material e $\mathbf{B}_{t^{\prime}}(t)$ é chamado tensor de Finger. $I_{1}$ e $I_{2}$ são invariantes de $\mathbf{B}_{t^{\prime}}(t)$, que serão definidos mais adiante.

A técnica numérica será desenvolvida usando o método de diferenças finitas baseado na metodologia GENSMAC [61] para a solução da equação de conservação de quantidade de movimento. Uma das dificuldades neste problema está no fato de que a equação constitutiva é dada na forma integral, o que traz a necessidade de conhecer a história de deformação em tempos anteriores para o cálculo das tensões. Essa história de deformação é determinada por um tensor $\mathbf{B}_{t^{\prime}}(t)$, chamado tensor de Finger, que traz informações sobre o deslocamento relativo das partículas de fluidos com o tempo. Calcular o tensor de Finger é portanto um dos problemas que devem ser atacados ao fazer simulações numéricas usando estes tipos de equações constitutivas, o que não ocorre com os modelos diferenciais.

Durante o desenvolvimento deste trabalho, procuramos na literatura uma técnica de cálculo do tensor de Finger que pudesse ser utilizada com o método de diferenças finitas. A maioria dos trabalhos utiliza uma abordagem lagrangiana para o cálculo do tensor de Finger (ou equivalentemente, o tensor de Cauchy) como será discutido no capítulo 2. No trabalho de Peters et al. [49], o tensor de Finger é obtido utilizando uma técnica euleriana, denominada método de campos de deformação, que foi implementada computacionalmente usando o método de elementos finitos. Neste trabalho, vamos fazer modificações nessa técnica para que a mesma possa ser utilizada com o método de diferenças finitas juntamente com a técnica Marker-and-Cell para simular escoamentos incompressíveis. Essas modificações se referem à forma como o tensor de Finger é determinado e como o tensor das tensões é calculado. Além disso, a aplicação do método de campos de deformação a escoamentos incompressíveis com superfícies livres é investigada.

Esta tese está organizada como a seguir:

O Capítulo 1 apresenta uma descrição do sistema Freeflow-2D e alguns conceitos que auxiliarão no entendimento da tese. No Capítulo 2, é desenvolvido um método numérico para calcular o tensor de Finger baseado nas idéias do método de campos de deformação. Uma revisão do método de campos de deformação original também é apre- 
sentada. Este capítulo termina com uma validação do método numérico apresentado utilizando as soluções exatas, obtidas com o cálculo das tensões para escoamentos em cisalhamento simples e escoamentos elongacionais uniaxiais pelo modelo integral de Maxwell. No Capítulo 3, descrevemos a implementação do método de campos de deformação para a solução de escoamentos incompressíveis bidimensionais no sistema FreeFlow-2D. São apresentados o método GENSMAC, as equações governantes, as condições de contorno e a discretização das equações por diferenças finitas. O Capítulo 4 apresenta a aplicação do sistema FreeFlow-2D na simulação de escoamentos incompressíveis bidimensionais. Utilizando o escoamento em um canal bidimensional, é realizada uma validação com a solução analítica obtida com o modelo de Maxwell e a convergência do método numérico desenvolvido nesta tese é demonstrada por meio de refinamento de malha. São apresentados os resultados para o problema clássico do escoamento em uma contração $4: 1$ utilizando os modelos Maxwell e K-BKZ. Uma comparação com dados experimentais para o problema da contração e também os resultados obtidos na simulação de escoamentos com superfícies livres: simulação do jato oscilante ("jet buckling") utilizando os modelos Maxwell e K-BKZ. 


\section{Capítulo 1}

\section{Conceitos Gerais}

\subsection{Desenvolvimentos de GENSMAC}

Em 1994, Tomé \& McKee introduziram a técnica GENSMAC (Generalized Simplified Marker-and-Cell) [61] para simular escoamentos newtonianos bidimensionais envolvendo superfícies livres em domínios arbitrários. GENSMAC é uma técnica numérica, baseada no método SMAC (Simplified Marker-and-Cell) [4], que utiliza o método de diferenças finitas numa malha deslocada e partículas marcadoras para representar o fluido. GENSMAC foi aplicado para simular escoamentos com superfícies livres tais como "enchimento de contêineres "e enchimento de cavidades com geometria complexa (ver figura 1.1). Mais tarde, o método GENSMAC foi estendido para escoamentos axissimétricos, onde o fluido é modelado por uma superfície fechada, utilizando partículas marcadoras somente na superfície do fluido [58]. As aplicações de GENSMAC para escoamentos axissimétricos incluem simulação de "splashing drop" e "enchimento de contêineres". A figura 1.2 mostra um exemplo de escoamentos axissimétricos realizados pelo código GENSMAC.

No entanto, muitos problemas em mecânica dos fluidos são inerentemente tridimensionais e isso tem motivado muitos pesquisadores a investigar técnicas numéricas para o tratamento de escoamentos tridimensionais envolvendo superfícies livres. Com este objetivo, Tomé et al. [57] desenvolveram o método GENSMAC3D para o tratamento de escoamentos incompressíveis tridimensionais de fluidos newtonianos, isotérmicos, com superfícies livres. O método GENSMAC3D foi implementado computacionalmente em um ambiente de simulação denominado FreeFlow-3D. Freeflow-3D [11] é um ambiente integrado para modelagem, simulação e visualização de escoamentos tridimensionais e é 

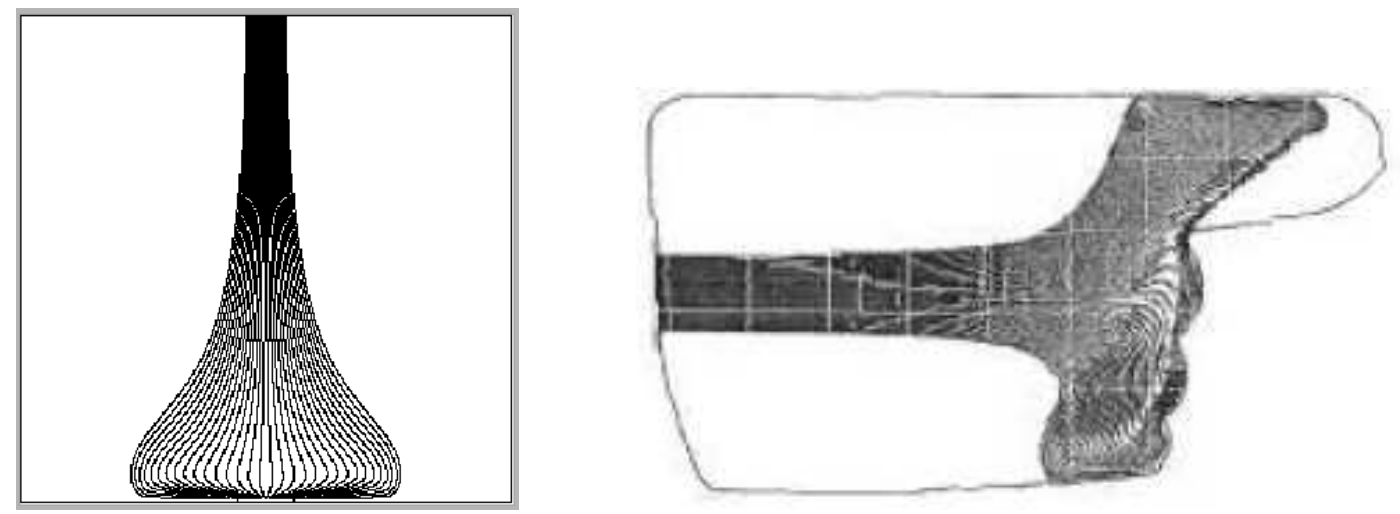

Figura 1.1: Simulação de enchimento de contêineres pelo código GENSMAC [61].
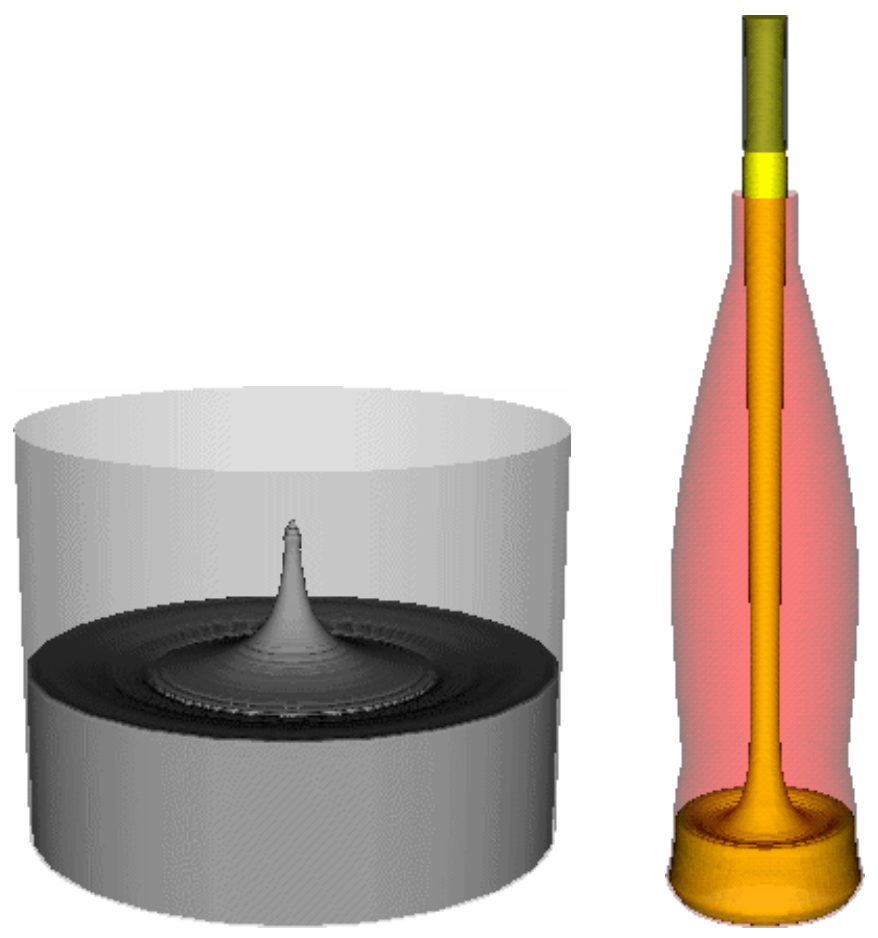

Figura 1.2: Simulação de enchimento de contêineres pelo código GENSMAC axissimétrico [58]. 
constituído por três módulos distintos: Modflow-3D, Simflow-3D e Visflow-3D. Este sistema foi estruturado para permitir futuras extensões nos três módulos. Em particular, o módulo Simflow-3D pode facilmente ser estendido para a incorporação de escoamentos de fluidos não-newtonianos, escoamentos multifásicos, escoamentos envolvendo movimento de contêineres, escoamentos turbulentos e escoamentos não-isotérmicos. A fim de investigar escoamentos em duas dimensões, uma versão bidimensional, denominada FreeFlow2D, foi desenvolvida [42]. FreeFlow-2D foi obtido de FreeFlow-3D pela supressão de uma coordenada cartesiana. Assim como FreeFlow-3D, o ambiente FreeFlow-2D é composto de três módulos que são responsáveis pela modelagem, simulação e visualização dos escoamentos. Recentemente, com o objetivo de investigar escoamentos de fluidos mais complexos, Tomé et al. [60] desenvolveram um algoritmo para tratar de escoamentos viscoelásticos utilizando o modelo Oldroyd-B, o qual foi incorporado no código GENSMAC 61 .

Inicialmente, o código FreeFlow-2D foi desenvolvido para simular escoamentos bidimensionais, incompressíveis e isotérmicos, de fluidos newtonianos. Atualmente os códigos FreeFlow-2D e FreeFlow-3D tratam de escoamentos bidimensionais e tridimensionais, não só de fluidos newtonianos mas também de alguns escoamentos de fluidos viscoelásticos governados por equações constitutivas diferenciais, por exemplo Oldroyd-B [60], Maxwell [15] e fluido de segunda ordem [28]. Ambos os códigos estão implementados em linguagem C, usando para a visualização o sistema de gerenciamento de janelas XView e são executados em sistemas Linux. As malhas utilizadas são cartesianas e estruturadas e estão em andamento projetos para migração do código para $\mathrm{C}++$, para um novo sistema de janelas e pretende-se ainda incluir malhas não estruturadas.

O objetivo principal desta tese é desenvolver método numérico para resolver os modelos integrais de Maxwell e K-BKZ e implementá-los no ambiente de simulação FreeFlow2D, para que o mesmo possa simular escoamentos viscoelásticos governados por esses modelos. 


\subsection{Alguns conceitos em reologia}

Nesta seção são apresentados alguns conceitos em reologia que julgamos necessários para o entendimento dos assuntos tratados mais adiante. As informações desta seção podem ser encontradas com mais detalhes nos livros de Bird et al. [8], Bretas [10], Macosko [34] e Tanner [56].

A reologia é a ciência que estuda a deformação e escoamento da matéria. Ela tem especial interesse no estudo das relações fundamentais entre força e deformação no material, em especial líquidos. Essas relações são denominadas equações constitutivas ou equações reológicas de estado.

As equações constitutivas caracterizam cada tipo de material, descrevendo o comportamento macroscópico resultante da sua constituição interna. Obviamente, não existe uma equação constitutiva que forneça todas as respostas experimentais de um dado material. Entretanto, a maioria dos materiais possuem comportamento que oscila entre dois extremos bem definidos que são o sólido ideal, que obedece a lei de Hooke, onde os tensores tensão e deformação estão relacionados de forma linear, e o fluido ideal newtoniano, determinado pela lei de viscosidade de Newton, que estabelece uma relação linear entre as tensões e gradientes de velocidade. Para fluidos incompressíveis esta relação pode ser representada por

$$
\tau^{i j}=\mu\left(\frac{\partial v_{j}}{\partial x_{i}}+\frac{\partial v_{i}}{\partial x_{j}}\right)
$$

com a constante $\mu$ sendo a viscosidade do fluido. Em notação vetorial essa equação toma a forma

$$
\tau=\mu \dot{\gamma}
$$

onde $\dot{\gamma}=\nabla \mathbf{v}+(\nabla \mathbf{v})^{t}$ é o tensor taxa de deformação.

Esses modelos constitutivos representam casos ideais. Entretanto, existem materiais que possuem características tanto de materiais sólidos quanto de materiais líquidos. Essa propriedade é conhecida como viscoelasticidade. Materiais como sangue, tintas e líquidos poliméricos apresentam esse comportamento viscoelástico. Em particular, os fluidos poliméricos são de grande interesse em processos industriais, dada a sua grande aplicabilidade. Esses fluidos são constituídos por macromoléculas com elevado peso molecular, levando ao aparecimento de comportamentos peculiares quando sujeitos a determinadas solicitações. As características não-newtonianas mais comuns são que a tensão é 
função não linear da taxa de deformação, a produção de tensões normais em cisalhamento e comportamentos elástico e viscoso.

Diferentemente dos fluidos newtonianos incompressíveis, nos fluidos poliméricos propriedades do material como a viscosidade podem não ser constantes e poderão depender também da taxa de cisalhamento e do tempo, dentre outros fatores. O conhecimento dessas funções materiais é de extrema importância pois servem para classificar os fluidos e podem ser usadas para determinar constantes em equações constitutivas específicas [8].

Os dois tipos de escoamento mais utilizados para caracterizar líquidos poliméricos são os cisalhantes e os escoamentos elongacionais.

O campo de velocidades de um escoamento em cisalhamento simples é dado por

$$
\begin{aligned}
& v_{x}=\dot{\gamma}_{y x} y, \\
& v_{y}=0 \\
& v_{z}=0
\end{aligned}
$$

com $\dot{\gamma}_{y x}=\frac{\partial v_{y}}{\partial x}$. Para este tipo de escoamento o tensor tensão total, $\boldsymbol{\sigma}=-p \mathbf{I}+\boldsymbol{\tau}$, é dado por

$$
\boldsymbol{\sigma}=\left(\begin{array}{ccc}
-p+\tau^{x x} & \tau^{x y} & 0 \\
\tau^{x y} & -p+\tau^{y y} & 0 \\
0 & 0 & -p+\tau^{z z}
\end{array}\right)
$$

Medidas experimentais mostram que nos escoamentos cisalhantes simples de fluidos incompressíveis apenas três medidas de tensões são possíveis e portanto de maior interesse: a tensão de cisalhamento, $\tau^{x y}$; a primeira diferença de tensões normais, $\tau^{x x}-\tau^{y y}$ e a segunda diferença de tensões normais, $\tau^{y y}-\tau^{z z}$, de modo que três funções materiais são de maior interesse em escoamentos cisalhantes simples, em regime permanente:

\section{Viscosidade não-newtoniana :}

$$
\eta(\dot{\gamma})=\frac{\tau^{x y}}{\dot{\gamma}_{x y}}
$$

Primeiro coeficiente de tensões normais:

$$
\Psi_{1}(\dot{\gamma})=\frac{\tau^{x x}-\tau^{y y}}{\left(\dot{\gamma}_{x y}\right)^{2}}
$$

Segundo coeficiente de tensões normais:

$$
\Psi_{2}(\dot{\gamma})=\frac{\tau^{y y}-\tau^{z z}}{\left(\dot{\gamma}_{x y}\right)^{2}}
$$


com $\dot{\gamma}=\left|\dot{\gamma}_{x y}\right|$.

Experimentalmente, a viscosidade é a função material mais bem conhecida e na maioria dos líquidos poliméricos a curva $\dot{\gamma} \times \eta$ apresenta duas regiões. Uma região a baixas taxas de cisalhamento, chamada platô newtoniano onde os valores da viscosidade independem da taxa de cisalhamento, e outra região a altas taxas de cisalhamento, chamada de região de lei de potências, onde a viscosidade decresce rapidamente com as taxas de cisalhamento. Quando $\dot{\gamma}$ tende a zero obtém-se $\eta(\dot{\gamma})=\eta_{0}$ chamada de viscosidade do platô newtoniano $([10])$. A altas taxas de cisalhamento, a viscosidade pode voltar a ser independente de $\dot{\gamma}$. Neste caso ela se aproxima de um valor constante que denotaremos por $\eta_{\infty}$. A figura 1.3 mostra um gráfico típico dessas regiões.

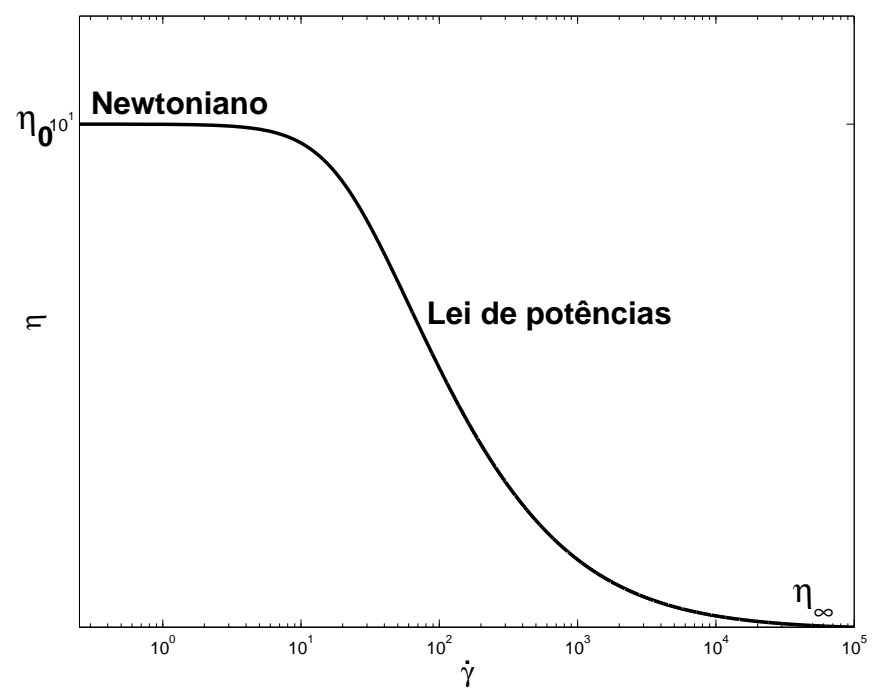

Figura 1.3: Gráfico típico mostrando a relação $\log \dot{\gamma} \times \log \eta$

Além das funções materiais em regime permanente, um fluido é ainda caracterizado por experimentos em regime transiente, nos quais novas funções materiais são obtidas. São exemplos:

\section{Crescimento de tensões antes de atingir o regime permanente de cisalhamento :}

Nesses escoamentos é imposta uma taxa de cisalhamento constante, $\dot{\gamma}_{0}$, e as tensões são medidas até que o estado estacionário seja alcançado. Neste caso determinamos uma propriedade reológica que indica o tempo necessário para atingir o valor no regime permanente. As funções materiais neste caso são análogas àquelas obtidas 
no regime permanente,

$$
\begin{aligned}
& \eta^{+}\left(t, \dot{\gamma}_{0}\right)=\frac{\tau^{x y}(t)}{\dot{\gamma}_{0}} \\
& \Psi_{1}^{+}\left(t, \dot{\gamma}_{0}\right)=\frac{\tau^{x x}(t)-\tau^{y y}(t)}{\left(\dot{\gamma}_{0}\right)^{2}} \\
& \Psi_{2}^{+}\left(t, \dot{\gamma}_{0}\right)=\frac{\tau^{y y}(t)-\tau^{z z}(t)}{\left(\dot{\gamma}_{0}\right)^{2}}
\end{aligned}
$$

Relaxação de tensões: Neste processo o fluido está sujeito a uma taxa de cisalhamento constante,$\dot{\gamma}_{0}$, e tem seu movimento subitamente interrompido. Então o decaimento do valor das tensões é observado. As funções materiais neste caso são

$$
\begin{aligned}
& \eta^{-}\left(t, \dot{\gamma}_{0}\right)=\frac{\tau^{x y}(t)}{\dot{\gamma}_{0}} \\
& \Psi_{1}^{-}\left(t, \dot{\gamma}_{0}\right)=\frac{\tau^{x x}(t)-\tau^{y y}(t)}{\left(\dot{\gamma}_{0}\right)^{2}} \\
& \Psi_{2}^{-}\left(t, \dot{\gamma}_{0}\right)=\frac{\tau^{y y}(t)-\tau^{z z}(t)}{\left(\dot{\gamma}_{0}\right)^{2}}
\end{aligned}
$$

Os escoamentos elongacionais têm campo de velocidades dado pela forma geral (ver Bird et al. [8])

$$
\begin{aligned}
& v_{x}=-\frac{1}{2} \dot{\varepsilon}(1+b) x, \\
& v_{y}=-\frac{1}{2} \dot{\varepsilon}(1-b) y, \\
& v_{z}=+\dot{\varepsilon} z
\end{aligned}
$$

em que $0 \leq b \leq 1$ e $\dot{\varepsilon}$ é a taxa de elongação, que pode depender do tempo. Para escolhas particulares do parâmetro $b$ temos os seguintes escoamentos:

- uniaxial se $b=0$ e $\dot{\varepsilon}>0$,

- biaxial se $b=0$ e $\dot{\varepsilon}<0$,

- planar se $b=1$.

Para esses tipos de escoamentos o tensor tensão total é dado por

$$
\boldsymbol{\sigma}=\left(\begin{array}{ccc}
-p+\tau^{x x} & 0 & 0 \\
0 & -p+\tau^{y y} & 0 \\
0 & 0 & -p+\tau^{z z}
\end{array}\right)
$$


As medidas de interesse são as duas diferenças de tensões normais e a função material mais utilizada é a viscosidade elongacional em regime permanente

$$
\bar{\eta}(\dot{\varepsilon})=\frac{\tau^{z z}-\tau^{x x}}{\dot{\varepsilon}} .
$$

Para mais detalhes e outras funções materiais em cisalhamento e em elongação veja, por exemplo, Bird et al. [8].

\subsection{Alguns modelos constitutivos integrais: Maxwell e $\mathrm{K}-\mathrm{BKZ}$}

Nesta tese estamos interessados na implementação de modelos integrais no ambiente de simulação FreeFlow-2D, especificamente os modelos integrais de Maxwell e K-BKZ (Kaye [24]; Bernstein, Kearsley e Zapas [7]). Esse modelos constitutivos fazem parte de um grupo maior de equações chamadas equações de Rivlin-Sawyers [55], que são da forma

$$
\boldsymbol{\tau}(t)=\int_{-\infty}^{t}\left\{\Psi_{1}\left(t-t^{\prime}, I_{1}, I_{2}\right) \mathbf{B}\left(t, t^{\prime}\right)+\Psi_{2}\left(t-t^{\prime}, I_{1}, I_{2}\right) \mathbf{C}\left(t, t^{\prime}\right)\right\} d t^{\prime}
$$

onde as funções $\Psi_{1}$ e $\Psi_{2}$ são funções escalares que dependem de $t, t^{\prime}$, com $-\infty<t^{\prime} \leq t$, e dos invariantes $I_{1}$ e $I_{2}$, definidos por

$$
\begin{aligned}
& I_{1}=\operatorname{tr}(\mathbf{B}), \\
& I_{2}=\operatorname{tr}(\mathbf{C}),
\end{aligned}
$$

onde $\mathbf{B}$ e $\mathbf{C}$ são os tensores de Finger e Cauchy-Green que serão definidos no próximo capítulo. Nesta tese iremos usar a forma fatorada da equação (1.10), onde supomos que as funções $\Psi_{i}\left(t-t^{\prime}, I_{1}, I_{2}\right), i=1,2$ podem ser escritas como um produto entre uma função temporal e uma função que depende da deformação, ou seja,

$$
\Psi_{i}\left(t-t^{\prime}, I_{1}, I_{2}\right)=M\left(t-t^{\prime}\right) \phi_{i}\left(I_{1}, I_{2}\right), i=1,2
$$

em que $M\left(t-t^{\prime}\right)$ é a função memória dada por

$$
M\left(t-t^{\prime}\right)=\sum_{k} \frac{a_{k}}{\lambda_{k}} e^{-\left(\frac{t-t^{\prime}}{\lambda_{k}}\right)},
$$

sendo $\lambda_{k}$ constantes temporais, chamadas tempos de relaxação, e $a_{k}$ são parâmetros que dependem do fluido. 
Neste caso a equação de (1.10) toma a forma

$$
\boldsymbol{\tau}(t)=\int_{-\infty}^{t} M\left(t-t^{\prime}\right)\left\{\phi_{1}\left(I_{1}, I_{2}\right) \mathbf{B}\left(t, t^{\prime}\right)+\phi_{2}\left(I_{1}, I_{2}\right) \mathbf{C}\left(t, t^{\prime}\right)\right\} d t^{\prime}
$$

Particularmente, usaremos o modelo dado por Papanastasiou, Scriven e Macosko [47] (modelo PSM), onde

$$
\begin{aligned}
\phi_{1}\left(I_{1}, I_{2}\right) & =\frac{\alpha}{\alpha-3+\beta I_{1}+(1-\beta) I_{2}} \\
\phi_{2}\left(I_{1}, I_{2}\right) & =0
\end{aligned}
$$

Neste caso $I_{1}=\operatorname{tr}(\mathbf{B})$ e $I_{2}=\frac{1}{2}\left[I_{1}^{2}-\operatorname{tr}\left(\mathbf{B}^{2}\right)\right]$ e os parâmetros $\alpha$ e $\beta$ dependem do material.

$\mathrm{Na}$ literatura são encontradas diversas variantes da equação (1.10). O próprio modelo PSM é referido como um modelo K-BKZ, embora o modelo K-BKZ tenha a forma fatorada dada por

$$
\boldsymbol{\tau}(t)=\int_{-\infty}^{t}\left\{\frac{\partial V\left(t-t^{\prime}, I_{1}, I_{2}\right)}{\partial I_{1}} \mathbf{B}\left(t, t^{\prime}\right)+\frac{\partial V\left(I_{1}, I_{2}\right)}{\partial I_{2}} \mathbf{C}\left(t, t^{\prime}\right)\right\} d t^{\prime}
$$

onde a função $V$ também depende de $t, t^{\prime}$, com $-\infty<t^{\prime} \leq t$, e dos invariantes $I_{1}$ e $I_{2}$. Deste modo podemos falar não de uma, mas de uma classe de equações tipo K-BKZ, dentre as quais está o modelo PSM que usaremos nesta tese.

O modelo convectivo superior de Maxwell é obtido fazendo $\phi_{1}\left(I_{1}, I_{2}\right)=1$ e $\phi_{2}\left(I_{1}, I_{2}\right)=$ 0 em (1.13), usando somente um tempo de relaxação na função memória, ou seja,

$$
\boldsymbol{\tau}(t)=\int_{-\infty}^{t} \frac{a}{\lambda} e^{-\frac{t-t^{\prime}}{\lambda}} \mathbf{B}\left(t, t^{\prime}\right) d t^{\prime}
$$

Nos capítulos que seguem vamos apresentar o desenvolvimento de um método numérico para a utilização desses modelos integrais no sistema Freeflow-2D. Entretanto, o método pode ser utilizado para outros modelos tipo K-BKZ, considerando a mesma função memória e com diferentes funções $\phi_{1}\left(I_{1}, I_{2}\right)$. 


\section{Capítulo 2}

\section{Solução Numérica dos modelos}

\section{integrais de Maxwell e K-BKZ}

Neste capítulo é apresentada uma abordagem diferente para a utilização do método de campos de deformação (Peters et al.[49], Hulsen et al. [22]), visando sua inclusão no código FreeFlow-2D com um esquema de diferenças finitas. Nesta abordagem, o cálculo da convecção do tensor de Finger, a fórmula de quadratura para a determinação da tensão e o modo de discretização do tempo passado são diferentes daqueles usados na forma original do método. Uma comparação entre os resultados numéricos e a solução exata da tensão para o modelo integral de Maxwell é apresentada no final do capítulo, onde são mostrados os resultados para fluxos em cisalhamento simples e em elongação uniaxial.

\subsection{O tensor de Finger}

Considere uma porção de material (por exemplo na forma de cubo) e os pontos $\mathbf{P}$ e $\mathbf{Q}$ nesse volume, conforme mostrado na figura 2.1. Seja $\mathbf{d} \mathbf{x}^{\prime}$ o vetor que associa os pontos $\mathbf{P}$ e $\mathbf{Q}$ num instante $t^{\prime}$. Após uma deformação, o vetor $\mathbf{d} \mathbf{x}^{\prime}$ tomará um nova configuração em relação ao seu estado de referência. A essa nova configuração num instante $t>t^{\prime}$ denotaremos por $\mathbf{d x}$.

Assumindo que $\mathbf{x}=\mathbf{x}\left(\mathbf{x}^{\prime}, t\right)$ seja uma função diferenciável de $\mathbf{x}^{\prime}$ e $t$, podemos relacionar a posição presente (no tempo $t$ ) com a posição de referência por

$$
\mathbf{d x}=\mathbf{F} \mathbf{d x}^{\prime}
$$

A quantidade $\mathbf{F}=\nabla \mathbf{x}=\frac{\partial \mathbf{x}}{\partial \mathbf{x}^{\prime}}$ é chamada gradiente de deslocamento e constitui 


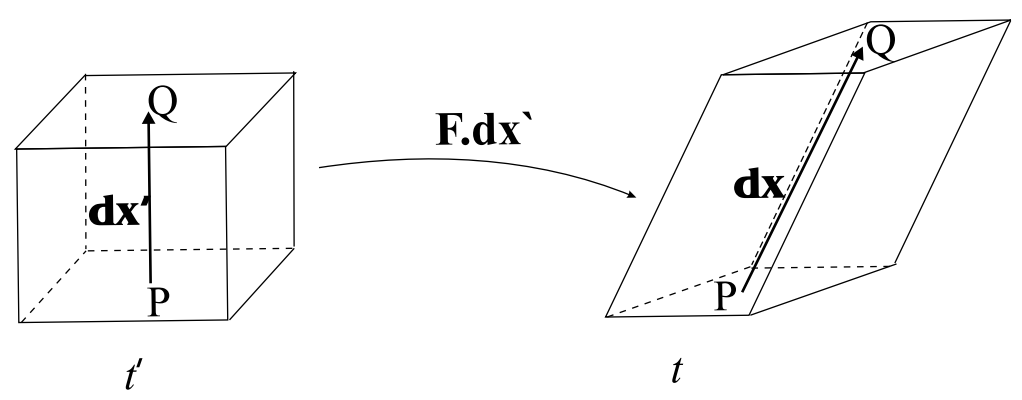

Figura 2.1: O tensor gradiente de deslocamento, $\mathbf{F}=\frac{\partial \mathbf{x}}{\partial \mathbf{x}^{\prime}}$, relaciona as configurações nos instantes $t^{\prime}$ e $t$.

um tensor de segunda ordem. Em coordenadas cartesianas $\mathbf{F}$ é dado por

$$
F_{i j}=\frac{\partial x_{i}}{\partial x_{j}^{\prime}}
$$

onde $x_{i}$ são as coordenadas de um ponto no estado deformado, no tempo presente $t$, e $x_{j}^{\prime}$ são as coordenadas no estado não deformado, num instante passado $t^{\prime}$.

Em geral, o tensor gradiente de deslocamento não é simétrico e contém informações tanto de deformações quanto de rotações e pode ser decomposto em um produto de tensores na forma

$$
\mathbf{F}=\mathbf{V R}
$$

em que $\mathbf{V}$ é simétrico e traz informações do esticamento (stretch) e $\mathbf{R}$, que é ortogonal e representa rotações de $\mathbf{d x}^{\prime}$ (ref. [0 $\left.{ }^{4} 4\right]$ ). Como $\mathbf{R R}^{T}=\mathbf{I}$, podemos eliminar as rotações em (2.2) multiplicando $\mathbf{F}$ por sua transposta:

$$
\mathbf{B}=\mathbf{F} \mathbf{F}^{T}=\mathbf{V} \mathbf{V}^{T}
$$

O tensor B é chamado tensor de Finger, que em coordenadas cartesianas é escrito como

$$
B_{i j}=\frac{\partial x_{i}}{\partial x_{k}^{\prime}} \frac{\partial x_{j}}{\partial x_{k}^{\prime}}
$$

onde estamos usando a notação de soma sobre índices repetidos. O tensor de Finger é o inverso do tensor de Cauchy-Green, C, que em coordenadas cartesianas é dado por

$$
C_{i j}=\frac{\partial x_{k}^{\prime}}{\partial x_{i}} \frac{\partial x_{k}^{\prime}}{\partial x_{j}}
$$

Estes tensores possuem as seguintes propriedades:

- são simétricos; 
- são positivos definidos ;

- $\operatorname{det} \mathbf{B}=\operatorname{det} \mathbf{C}=1$ em escoamentos incompressíveis.

Ao simular escoamentos de fluidos utilizando equações constitutivas tipo K-BKZ a maior dificuldade é a determinação do tensor de Finger. A maioria dos trabalhos existentes na literatura trata esse problema de uma forma lagrangiana, de modo que a posição das partículas é calculada para a obtenção do tensor gradiente de deslocamento $\mathbf{F}$ e posterior obtenção do tensor de Finger, $\mathbf{B}=\mathbf{F F}^{T}$.

Um dos primeiros trabalhos a usar uma formulação integral de equações constitutivas em simulações numéricas foi apresentado por Viriyayuthakorn e Caswell [65]. Para a obtenção do tensor de Finger a estratégia utilizada foi determinar o tensor gradiente de deslocamento por meio da relação

$$
\mathbf{F}=\mathbf{I}+\nabla \mathbf{U}^{T}
$$

em seguida calcular o tensor de Cauchy, $\mathbf{C}=\mathbf{F}^{T} \mathbf{F}$, e finalmente o tensor e Finger $\mathbf{B}=$ $\mathbf{C}^{-1}$. Nesta equação $\mathbf{U}$ é o deslocamento vetorial da partícula relativa à sua posição $\mathbf{r}$ no tempo presente $t$. A fim de calcular o tensor $\mathbf{C}$ nos pontos da quadratura, o vetor deslocamento deve ser calculado através de uma integral ao longo do caminho da partícula. O deslocamento $\mathbf{U}\left(\mathbf{r}, t^{\prime}-\Delta s\right)$ é dado por

$$
\mathbf{U}\left(\mathbf{r}, t^{\prime}-\Delta s\right)=\mathbf{U}\left(\mathbf{r}, t^{\prime}\right)-\Delta s \mathbf{V}\left(\mathbf{r}, t^{\prime}\right)+\frac{(\Delta s)^{2}}{2} \dot{\mathbf{V}}\left(\mathbf{r}, t^{\prime}\right)+O\left(\Delta s^{3}\right)
$$

onde $\dot{\mathbf{V}}$ é a derivada de $\mathbf{V}$ com relação ao tempo.

Luo e Tanner [32, 33] propuseram um método baseado em elementos de linha de corrente para simular escoamentos usando o modelo Maxwell em sua forma diferencial [32] e na sua forma integral [33]. No que se refere ao caso integral, a determinação do tensor $\mathbf{C}$ em cada ponto da quadratura é feita pelo cálculo de $\mathbf{F}$ usando a relação $\mathbf{C}=\mathbf{F}^{T} \mathbf{F}$. F é determinado pela integração numérica da equação

$$
\left\{\begin{array}{l}
\frac{D \mathbf{F}\left(t-t^{\prime}\right)}{D t^{\prime}}=\mathbf{L}\left(t^{\prime}\right) \mathbf{F}\left(t-t^{\prime}\right) \\
\mathbf{F}\left(t-t^{\prime}\right)_{t^{\prime}=t}=\mathbf{I}
\end{array}\right.
$$

ao longo das linhas de corrente. $\mathbf{L}\left(t^{\prime}\right)$ é o gradiente de velocidade sobre a linha de corrente na posição ocupada pela partícula no instante $t^{\prime}$. A configuração presente foi tomada como referência e a deformada é tomada em $t^{\prime}$. A integração numérica é feita com um método 
de Runge-Kutta. Além do modelo de Maxwell, a equação K-BKZ também foi testada, obtendo bons resultados para escoamentos cisalhantes. Para escoamentos elongacionais, os resultados foram menos precisos. Um problema apontado é que o método não é capaz de descrever zonas de recirculação.

Para problemas envolvendo recirculações Luo e Mitsoulis [31] desenvolveram um método usando elementos convencionais ao invés daqueles baseados em linhas de corrente. Ao invés do método de Runge-Kutta, a integração da equação (2.6) é feita pelo método de Euler modificado

$$
\mathbf{F}\left(t^{\prime}\right)=\mathbf{I}-\frac{d s}{2}\left[\mathbf{L}+\mathbf{L}^{\prime}-d s \mathbf{L}^{\prime} \mathbf{L}\right]+O\left(d s^{3}\right)
$$

Este método serviu de base para muitos trabalhos subseqüentes na literatura como os de Luo e Mitsoulis [30], Olley [44] e recentemente Mitsoulis et al. [39].

A técnica numérica usada nesta tese para o cálculo do tensor tensão $\tau$ é baseada nas idéias do método de campos de deformação, introduzida por Peters et al. [49] e Hulsen et al. [22]. Entretanto, a metodologia utilizada difere daquela utilizada por Peters et al. [49] e Hulsen et al. [22] em três pontos:

i) Procedimento para a discretização do instante passado $t^{\prime}$.

ii) Técnica numérica utilizada para o cálculo do tensor de Finger,

ii) Método numérico utilizado para a solução da integral.

Antes de apresentarmos os detalhes do método numérico desenvolvido nesse trabalho, vamos descrever as idéias originais do método numérico de Peters et al. [49] e Hulsen et al. [22].

Chamaremos de campo de deformação ao conjunto de tensores de Finger $\mathbf{B}_{t_{i}^{\prime}}(t)$, avaliados em um número finito de instantes $t_{i}^{\prime}$ do tempo passado $t_{i}^{\prime} \leq t$. Esse conjunto discreto de deformações será usado para descrever a história de deformação até o instante presente.

\subsection{Forma original do método de campos de deformação}

Originalmente, o método dos campos de deformação foi proposto para simular escoamentos confinados de fluidos cuja equação constitutiva era o modelo Rivlin-Sawyers, dado 
por

$$
\boldsymbol{\tau}(\mathbf{x}, t)=\int_{-\infty}^{t} M\left(t-t^{\prime}\right) \mathbf{f}\left[\mathbf{B}_{t^{\prime}}(\mathbf{x}, t)\right] d t^{\prime}
$$

em que $M\left(t-t^{\prime}\right)$ é a função memória e $\mathbf{B}_{t^{\prime}}(\mathbf{x}, t)$ é o tensor de Finger, tomado com relação a uma configuração no instantes passado $t^{\prime}$, de um elemento de fluido que está no instante presente, $t$, na posição $\mathbf{x} . \mathbf{f}\left[\mathbf{B}_{t^{\prime}}(\mathbf{x}, t)\right]$ é uma função que fornece informações sobre a deformação do material . O tensor de Finger $\mathbf{B}_{t^{\prime}}(\mathbf{x}, t)$ é armazenado em diferentes instantes passados $t_{i}^{\prime}$ e em seguida convectados segundo a equação

$$
\frac{\partial}{\partial t} \mathbf{B}_{t_{i}^{\prime}}(\mathbf{x}, t)+\mathbf{v}(\mathbf{x}, t) \cdot \nabla \mathbf{B}_{t_{i}^{\prime}}(\mathbf{x}, t)=[\nabla \mathbf{v}(\mathbf{x}, t)]^{T} \cdot \mathbf{B}_{t_{i}^{\prime}}(\mathbf{x}, t)+\mathbf{B}_{t_{i}^{\prime}}(\mathbf{x}, t) \cdot \nabla \mathbf{v}(\mathbf{x}, t),
$$

com a condição $\mathbf{B}_{t}(t)=\mathbf{I}$. No trabalho de Peters et al. [49] é usado o método de Euler de primeira ordem para aproximar a derivada temporal. Para a discretização espacial das equações de conservação de quantidade de movimento e da continuidade é usado um esquema de elementos finitos, no qual é empregada a formulação DEVSS (discrete elasticviscous split stress), de Guénette e Fortin [20], para separar a contribuição elástica e viscosa da tensão. O método de Galerkin descontínuo é utilizado para obter a discretização da equação (2.9).

A divisão do intervalo $-\infty \leq t^{\prime} \leq t$ em um número finito de instantes é obtida pela discretização do tempo decorrido $s=t-t^{\prime}$, de modo que $t_{i}^{\prime}=t-s_{i}$. Essa discretização não é uniforme, uma vez que a função memória assume valores pequenos para $t^{\prime}$ muito afastados do instante atual $t$. O tempo decorrido $s_{c}$, no qual $t^{\prime}$ é truncado, é escolhido como sendo um certo número de vezes o tempo de relaxação, e os intervalos entre os pontos $s_{i}$ têm tamanhos múltiplos do passo de tempo $\delta t$. Em geral intervalos entre tempos mais distantes do tempo atual têm tamanho dobrado em relação ao intervalo anterior.

A função $\mathbf{f}\left[\mathbf{B}_{t^{\prime}}(\mathbf{x}, t)\right]$ é aproximada em $t^{\prime}$ por interpolações entre os campos criados nos instantes $t-s_{i}$. A função aproximada é escrita como

$$
\mathbf{f}\left[\mathbf{B}_{t-s}(t)\right]=\sum_{i=0}^{N-1} \mathbf{f}\left[\mathbf{B}_{t-s_{i}}(t)\right] \phi_{i}(s),
$$

onde $\phi_{i}(s)$ são funções lineares (ver figura 2.2 ).

Uma vez obtida a aproximação para a função $\mathbf{f}\left[\mathbf{B}_{t^{\prime}}(\mathbf{x}, t)\right]$, é feita a substituição de (2.10) na equação (2.8), sendo que antes disso é feita a mudança de variável $s=t-t^{\prime}$. O resultado é dado pela expressão

$$
\boldsymbol{\tau}(\mathbf{x}, t)=\sum_{i=0}^{N-1} W_{i} \mathbf{f}\left[\mathbf{B}_{t-s_{i}}(t)\right],
$$




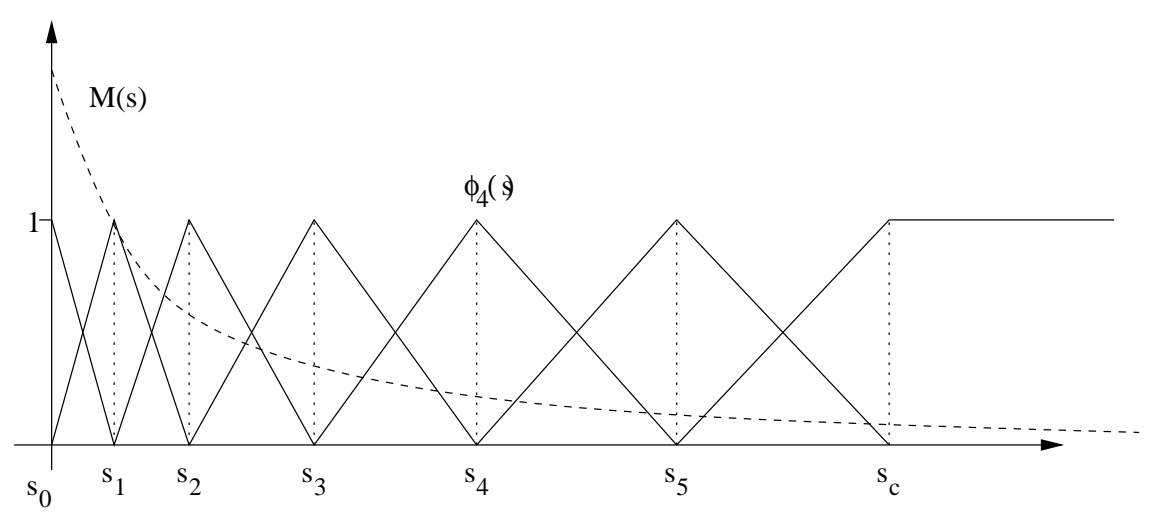

Figura 2.2: Funções de interpolação usadas por Peters et al. [49]. $M(s)$ é a função memória, com $s=t-t^{\prime}$ e $s_{c}$ é o tempo de corte.

com

$$
W_{i}=\int_{0}^{\infty} M(s) \phi_{i}(s) d s .
$$

Neste caso, os pesos $W_{i}$ são calculados somente uma vez, antes do início da simulação, e devem ser associados ao correspondente campo $\mathbf{B}_{t-s_{i}}(t)$ em cada passo de tempo.

Considerando que o passo de tempo $\delta t$ seja o mesmo para a discretização das equações de quantidade de movimento e para o cálculo da integral, temos os seguintes passos para a atualização dos campos de deformação:

1. Atualizar os campos existentes.

$$
\mathbf{B}_{t-i \delta t}(t) \longrightarrow \mathbf{B}_{t-i \delta t}(t+\delta t)
$$

Isso deve ser feito com o auxilio da equação (2.9).

2. Anular um campo antigo. Na técnica mais simples, discretização equidistante, o campo mais velho será anulado.

$$
\mathbf{B}_{t-s_{c}} \longrightarrow N U L L
$$

3. Criação de um novo campo. Usando o espaço de memória deixado pelo anulamento do campo mais antigo, um novo campo é criado como um campo tensorial unitário com relação ao tempo $t+\delta t$.

$$
\mathbf{B}_{t+\delta t}(t+\delta t):=\mathbf{I}
$$

Após o processo de convecção dos tensores de Finger, os pesos $W_{i}$ devem ser atualizados para os campos correspondentes (ver figura 2.3 ). 


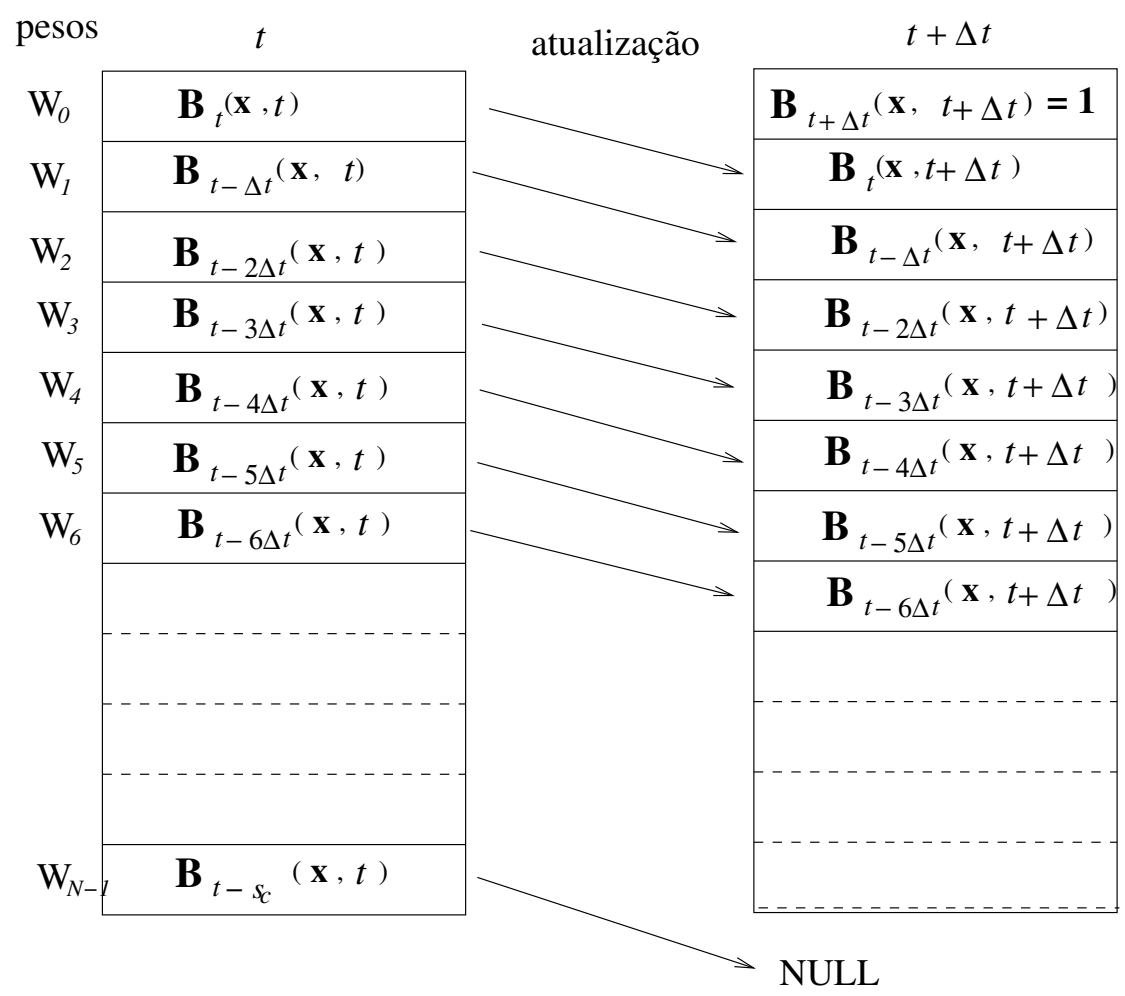

Figura 2.3: Processo de atualização de Peters et al. [49] para o caso em que $\delta s=\delta t$. A cada passo de tempo um novo campo é criado e os pesos $W_{i}$ são atualizados.

Hulsen et al. 22] observaram os seguintes problemas com o método acima:

- Os campos têm que ser removidos e criados, o que leva a um difícil manuseio de memória;

- Os campos não tornam-se estacionários, mesmos que o fluxo o seja;

- Embora a malha em $t^{\prime}$ seja mais refinada próxima do instante atual $t$, ela está sempre ligada ao passo de tempo;

- O método apresenta instabilidades que não ocorrem ao resolver o problema diferencial equivalente.

Os autores afirmam que os três primeiros problemas têm como causa o fato de $t^{\prime}$ ser usado como referência nos campos e propõem, ao invés disso, que seja usado o tempo decorrido $s=t-t^{\prime}$ como referência. Com isso é feita a seguinte mudança:

$$
\frac{\partial}{\partial t} \mathbf{B}_{t^{\prime}}(\mathbf{x}, t)=\left.\frac{\partial}{\partial t} \mathbf{B}_{t^{\prime}}(\mathbf{x}, t, s)\right|_{t^{\prime}, \mathbf{x}}=\frac{\partial \mathbf{B}}{\partial t}+\left.\frac{\partial \mathbf{B}}{\partial s} \frac{\partial s}{\partial t}\right|_{t^{\prime}}=\frac{\partial \mathbf{B}}{\partial t}+\frac{\partial \mathbf{B}}{\partial s}
$$


Agora, ao invés da equação (2.9), deve-se resolver

$$
\frac{\partial \mathbf{B}_{t-s}}{\partial t}+\frac{\partial \mathbf{B}_{t-s}}{\partial s}=-\mathbf{v} \cdot \nabla \mathbf{B}_{t-s}+(\nabla \mathbf{v})^{T} \cdot \mathbf{B}_{t-s}+\mathbf{B}_{t-s} \cdot \nabla \mathbf{v}
$$

com as condições $\mathbf{B}(\mathbf{x}, 0, s)=\mathbf{I}$ e $\mathbf{B}(\mathbf{x}, t, 0)=\mathbf{I}$. Ainda segundo os autores, o quarto problema também desaparece, mas afirma-se que não há uma explicação clara para esse fato.

A discretização do tempo decorrido $s=t-t^{\prime}$ é dado por $\Delta s_{i+1}=f \Delta s_{i}$, onde $\Delta s_{i}=$ $s_{i+1}-s_{i}$ e $f$ é um fator encontrado por iterações, conhecidos $\Delta s_{0}, N$ (número de campos usados) e $s_{c}=s_{N}$. Um valor ótimo para $\Delta s_{0}$ é dado aproximadamente por

$$
\frac{\Delta s_{0}^{o p t}}{\lambda}=\frac{3.1}{N}
$$

\subsection{Cálculo do tensor das tensões $\boldsymbol{\tau}(t)$}

Tanto a forma original do método de campos de deformação, introduzida por Peters et al. [49], quanto a forma melhorada proposta por Hulsen et al. [22] têm como característica comum o fato de que a váriável usada na integração das tensões é o tempo decorrido $s=t-t^{\prime}$, de modo que a tensão é obtida por

$$
\boldsymbol{\tau}(t)=\int_{0}^{\infty} M(s) \mathbf{f}\left[\mathbf{B}_{t-s}(t)\right] d s,
$$

e portanto a dependência da tensão com o tempo presente $t$ não aparece explicitamente na integral.

Ao fazer esta mudança de variável deve-se tomar cuidado para não inferir erradamente que o tensor de finger $\mathbf{B}_{t^{\prime}}(t)$ é função somente do tempo decorrido $s=t-t^{\prime}$, o que não é verdade no caso geral. Consideremos como exemplo o cálculo da componente $\tau_{x y}(t)$ antes de atingir o regime permanente de cisalhamento, usando como equação constitutiva o modelo integral de Maxwell

$$
\boldsymbol{\tau}(t)=\int_{-\infty}^{t} \frac{a}{\lambda} e^{-\frac{t-t^{\prime}}{\lambda}} \mathbf{B}_{t^{\prime}}(t) d t^{\prime}
$$

Neste caso é imposta uma taxa de cisalhamento constante $\dot{\gamma}$ e vamos calcular a expressão para o crescimento de tensões com o tempo. A história de deformação é dada por [8, 47]

$$
\begin{array}{ll}
B^{x y}=\dot{\gamma}\left(t-t^{\prime}\right) & t>0, \quad t^{\prime}>0 \\
B^{x y}=\dot{\gamma} t & t>0, \quad t^{\prime}<0 .
\end{array}
$$


A forma mais natural de determinar a expressão para $\tau_{x y}$ é fazer a integração nos intervalos $[-\infty, 0]$ e $[0, t]$, nos quais o tensor de Finger assume formas distintas, ou seja, resolvemos a equação

$$
\tau_{x y}(t)=\int_{-\infty}^{0} \frac{a}{\lambda} e^{\left(-\frac{t-t^{\prime}}{\lambda}\right)}(\dot{\gamma} t) d t^{\prime}+\int_{0}^{t} \frac{a}{\lambda} e^{\left(-\frac{t-t^{\prime}}{\lambda}\right)} \dot{\gamma}\left(t-t^{\prime}\right) d t^{\prime}
$$

Com base nessa idéia nossa abordagem tomará o tempo $t^{\prime}$ como referência, de modo que integrais do tipo (2.17) serão resolvidas a cada passo de tempo.

A idéia central é a mesma, ou seja, determinar o tensor de Finger em um número finito de instantes passados $t^{\prime}$ e convectá-los com o auxilio da equação (2.9), que relembramos aqui,

$$
\frac{\partial}{\partial t} \mathbf{B}_{t^{\prime}}(\mathbf{x}, t)+\mathbf{v}(\mathbf{x}, t) \cdot \nabla \mathbf{B}_{t^{\prime}}(\mathbf{x}, t)=[\nabla \mathbf{v}(\mathbf{x}, t)]^{T} \cdot \mathbf{B}_{t^{\prime}}(\mathbf{x}, t)+\mathbf{B}_{t^{\prime}}(\mathbf{x}, t) \cdot \nabla \mathbf{v}(\mathbf{x}, t)
$$

A seguir faremos uma abordagem diferente no que se refere à discretização do instante passado $\left(t^{\prime}\right)$, ao avanço do tensor de Finger para um tempo posterior e ao cálculo da integral que define a tensão na equação constitutiva.

\subsubsection{Discretização do tempo passado $t^{\prime}$}

Como a função memória que usamos é dada por uma exponencial ou soma de exponenciais, $M\left(t-t^{\prime}\right)=\sum_{m} \frac{a_{m}}{\lambda_{m}} e^{\frac{-\left(t-t^{\prime}\right)}{\lambda_{m}}}$, o procedimento para o discretização de $t^{\prime}$ é simplificado. Para tornar a explicação mais clara, vamos considerar primeiro o caso de termos somente uma exponencial na função memória.

A questão fundamental ao dividir o intervalo $[0, t]$ em subintervalos $\left[t_{i-1}^{\prime}, t_{i}^{\prime}\right]$ é saber qual o espaçamento apropriado entre os pontos $t_{i}^{\prime}$, já que a função memória é dada por uma exponencial, e portanto valores de $t^{\prime}$ muito afastados de $t$ têm pouca contribuição no cálculo das tensões. Uma forma direta de obter esses pontos é utilizar os valores da imagem inversa da função memória. Sendo $M\left(t-t^{\prime}\right)$ uma função exponencial, fazer isso é muito simples. Seja $M\left(t_{n}, t^{\prime}\right)$ a configuração da função memória no instante $t_{n}$. Por enquanto, vamos considerar $M\left(t_{n}, t^{\prime}\right)=\frac{a}{\lambda} e^{\left(-\frac{t_{n}-t^{\prime}}{\lambda}\right)}, t^{\prime} \leq t_{n}$. Dividindo o intervalo $\left[M\left(t_{n}, 0\right), M\left(t_{n}, t_{n}\right)\right]$ em $\mathrm{N}$ subintervalos igualmente espaçados, teremos $\mathrm{N}+1$ pontos com espaçamento $d M=\frac{M\left(t_{n}, t_{n}\right)-M\left(t_{n}, 0\right)}{N}$ entre eles. Esses pontos são dados por $M_{k}\left(t_{n}\right)=$ $M\left(t_{n}, 0\right)+k \cdot d M, k=0,1,2, \cdots, N$. Agora podemos determinar a discretização do 
intervalo $\left[0, t_{n}\right]$ tomando a imagem inversa do valor da função memória $M_{k}\left(t_{n}\right)$, obtendo

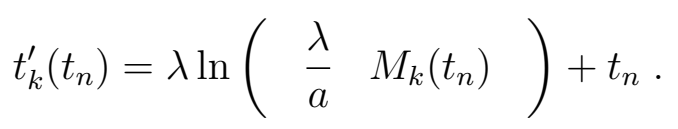

Esses valores são calculados em cada passo de tempo. Quando $M\left(t_{n}, 0\right) \leq \varepsilon, \operatorname{com} \varepsilon>0$ pequeno, o intervalo considerado para determinação de $d M$ será $\left[\varepsilon, M\left(t_{n}, t_{n}\right)\right]$. Isso evita que para tempos muito grandes estejamos usando valores em $t^{\prime}$ s que não irão contribuir significativamente para o cálculo das tensões.

Para funções de memória com múltiplos modos de relaxação, encontrar a imagem inversa diretamente é um pouco mais difícil, já que é necessário resolver uma equação exponencial. Ao invés disso, encontramos um valor aproximado usando o procedimento acima para cada termo da função e tomamos a média dos valores encontrados. Assim, se $M\left(t_{n}-t^{\prime}\right)=\sum_{m} \frac{a_{m}}{\lambda_{m}} e^{\frac{-\left(t_{n}-t^{\prime}\right)}{\lambda_{m}}}$, procedemos como antes para cada $M_{m}\left(t_{n}-t^{\prime}\right)=\frac{a_{m}}{\lambda_{m}} e^{\frac{-\left(t_{n}-t^{\prime}\right)}{\lambda_{m}}}$ e encontramos

$$
t_{k_{m}}^{\prime}\left(t_{n}\right)=\lambda_{m} \ln \left(\frac{\lambda_{m}}{a_{m}} M_{k_{m}}\left(t_{n}\right)\right)+t_{n},
$$

$\operatorname{com} M_{k_{m}}\left(t_{n}\right)=M_{m}\left(t_{n}, 0\right)+k \cdot d M_{m}, \quad k=0,1,2, \cdots, N$ e $d M_{m}=\frac{M_{m}\left(t_{n}, t_{n}\right)-M_{m}\left(t_{n}, 0\right)}{N}$. Portanto

$$
t_{k}^{\prime}\left(t_{n}\right)=\frac{\sum_{m=1}^{q} t_{k_{m}}^{\prime}\left(t_{n}\right)}{q}
$$

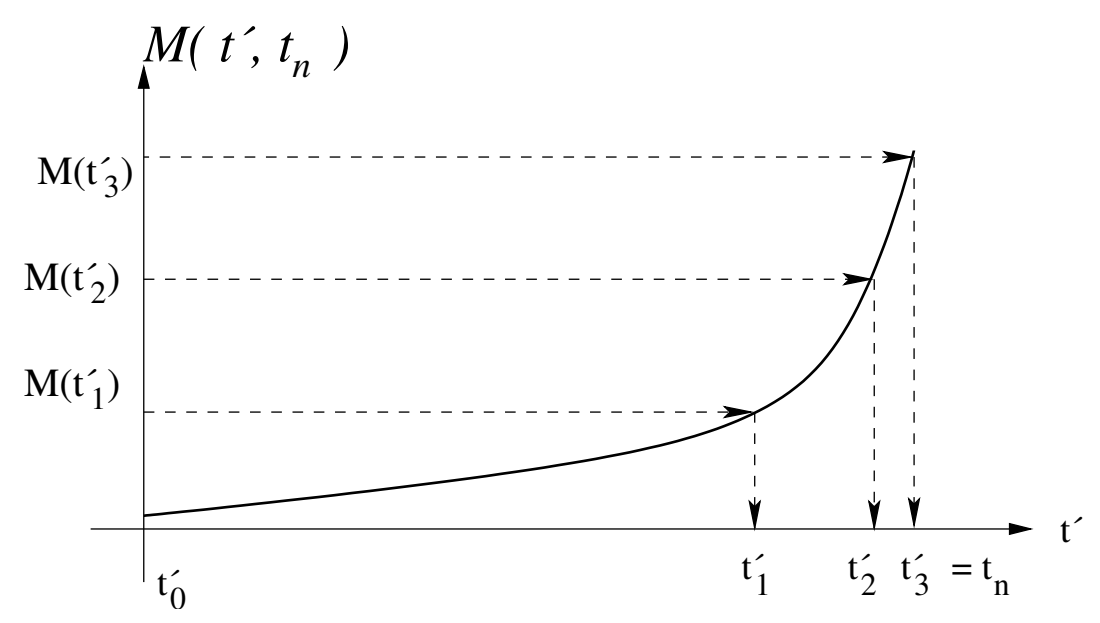

Figura 2.4: Discretização do intervalo $\left[0, t_{n}\right]$. Os valores $t_{i}^{\prime}$ são obtidos pela imagem inversa dos pontos $M\left(t_{i}^{\prime}\right)$ 


\subsubsection{Convecção dos tensores de Finger}

O avanço no tempo do tensor de Finger é feito através das equações (2.9). Em duas dimensões, essas equações são dadas por

$$
\begin{gathered}
\frac{\partial}{\partial t} B_{t^{\prime}}^{x x}+\frac{\partial}{\partial x}\left(u B_{t^{\prime}}^{x x}\right)+\frac{\partial}{\partial y}\left(v B_{t^{\prime}}^{x x}\right)=2\left[\frac{\partial u}{\partial x} B_{t^{\prime}}^{x x}+\frac{\partial u}{\partial y} B_{t^{\prime}}^{x y}\right], \\
\frac{\partial}{\partial t} B_{t^{\prime}}^{x y}+\frac{\partial}{\partial x}\left(u B_{t^{\prime}}^{x y}\right)+\frac{\partial}{\partial y}\left(v B_{t^{\prime}}^{x y}\right)=\frac{\partial u}{\partial y} B_{t^{\prime}}^{y y}+\frac{\partial v}{\partial x} B_{t^{\prime}}^{x x} \\
\frac{\partial}{\partial t} B_{t^{\prime}}^{y y}+\frac{\partial}{\partial x}\left(u B_{t^{\prime}}^{y y}\right)+\frac{\partial}{\partial y}\left(v B_{t^{\prime}}^{y y}\right)=2\left[\frac{\partial v}{\partial x} B_{t^{\prime}}^{x y}+\frac{\partial v}{\partial y} B_{t^{\prime}}^{y y}\right] .
\end{gathered}
$$

Para o avanço no tempo usaremos o método de Euler explícito. Dado o campo de deformação para o tempo $t_{n}$, o avanço para o tempo $t_{n+1}$ é dado por

$$
\begin{aligned}
\mathbf{B}_{t_{k}^{\prime}\left(t_{n}\right)}\left(\mathbf{x}, t_{n+1}\right)= & \mathbf{B}_{t_{k}^{\prime}\left(t_{n}\right)}\left(\mathbf{x}, t_{n}\right)+\delta t\left\{-\mathbf{v}\left(\mathbf{x}, t_{n}\right) \cdot \nabla \mathbf{B}_{t_{k}^{\prime}\left(t_{n}\right)}\left(\mathbf{x}, t_{n}\right)\right. \\
& \left.+\left[\nabla \mathbf{v}\left(\mathbf{x}, t_{n}\right)\right]^{T} \cdot \mathbf{B}_{t_{k}^{\prime}\left(t_{n}\right)}\left(\mathbf{x}, t_{n}\right)+\mathbf{B}_{t_{k}^{\prime}\left(t_{n}\right)}\left(\mathbf{x}, t_{n}\right) \cdot \nabla \mathbf{v}\left(\mathbf{x}, t_{n}\right)\right\}
\end{aligned}
$$

No entanto deve-se observar que isto fornece o campo nos tempos $t_{k}^{\prime}\left(t_{n}\right), \mathbf{B}_{t_{k}^{\prime}\left(t_{n}\right)}\left(\mathbf{x}, t_{n+1}\right)$. É necessário ainda calcular o tensor de Finger nos tempos $t_{k}^{\prime}\left(t_{n+1}\right)$. Para isso identifica-se em qual intervalo $\left[t_{i}^{\prime}\left(t_{n}\right), t_{i+1}^{\prime}\left(t_{n}\right)\right], i=0, \ldots, N-1$, o valor $t_{k}^{\prime}\left(t_{n+1}\right)$ está e em seguida determina-se o valor do tensor de Finger neste ponto. Se, por exemplo, $t_{\xi}^{\prime}\left(t_{n+1}\right) \in$ $\left[t_{k}^{\prime}\left(t_{n}\right), t_{k+1}^{\prime}\left(t_{n}\right)\right]$, o tensor $\mathbf{B}_{t_{\xi}^{\prime}\left(t_{n+1}\right)}\left(\mathbf{x}, t_{n+1}\right)$ é obtido utilizando uma fórmula de interpolação polinomial de segunda ordem, usando os valores $\mathbf{B}_{t_{k-1}^{\prime}\left(t_{n}\right)}\left(\mathbf{x}, t_{n+1}\right), \mathbf{B}_{t_{k}^{\prime}\left(t_{n}\right)}\left(\mathbf{x}, t_{n+1}\right)$, $\mathbf{B}_{t_{k+1}^{\prime}\left(t_{n}\right)}\left(\mathbf{x}, t_{n+1}\right)$. A figura 2.5 mostra como a convecção é feita tomando como exemplo a componente $B_{x y}$ do tensor de Finger em cisalhamento simples, $B^{x y}=\dot{\gamma}\left(t-t^{\prime}\right)$.

Este procedimento de interpolação é importante, pois faz com que os valores do tensor de Finger nos novos tempos $t_{k}^{\prime}\left(t_{n+1}\right)$ sejam corretamente aproximados. Sem isto, estaríamos aproximando os valores do tensor de Finger nos tempos $t_{k}^{\prime}\left(t_{n+1}\right)$ pelos obtidos nos tempos $t_{k}^{\prime}\left(t_{n}\right)$, como mostra a figura 2.6. A vantagem desse procedimento em fluxos em cisalhamento simples é imediata, pois neste caso o erro no cálculo da tensão terá apenas a contribuição do erro decorrente da aproximação do tensor de Finger pelo método de Euler, já que a interpolação e fórmula de quadratura (ver seção 2.3.3) serão de segunda ordem em $t^{\prime}$. E ainda será independente do número de pontos $t_{k}^{\prime}$ (número de campos na terminologia de Peters et al. [49]) que se use, como veremos mais adiante com o modelo de Maxwell. 


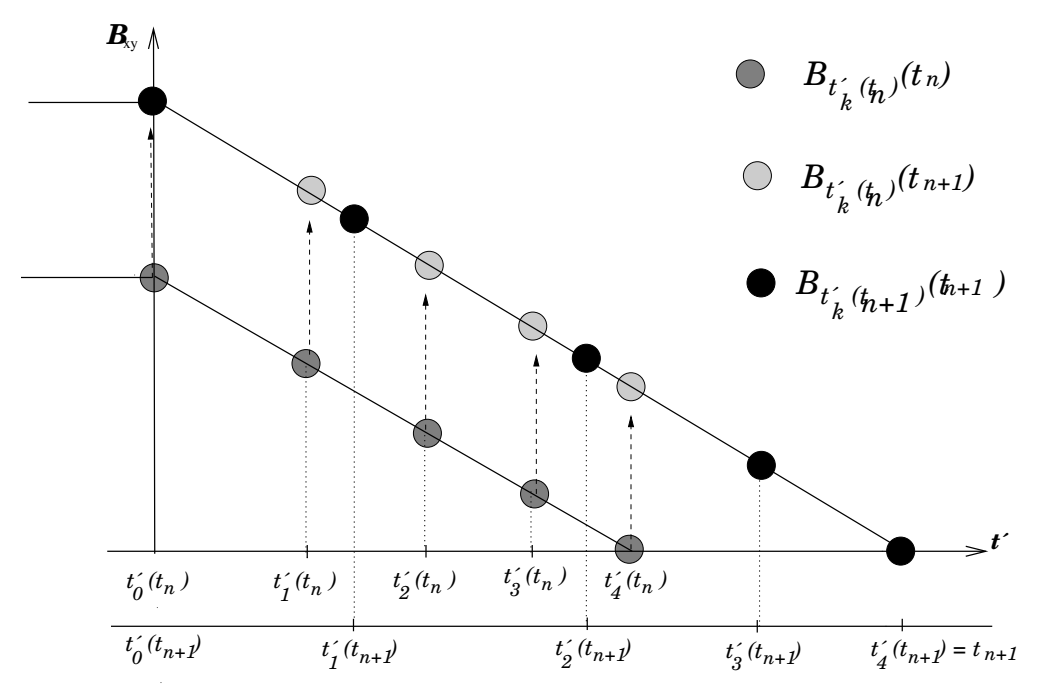

Figura 2.5: Cálculo do tensor de Finger nos tempos $t_{k}^{\prime}\left(t_{n+1}\right)$.

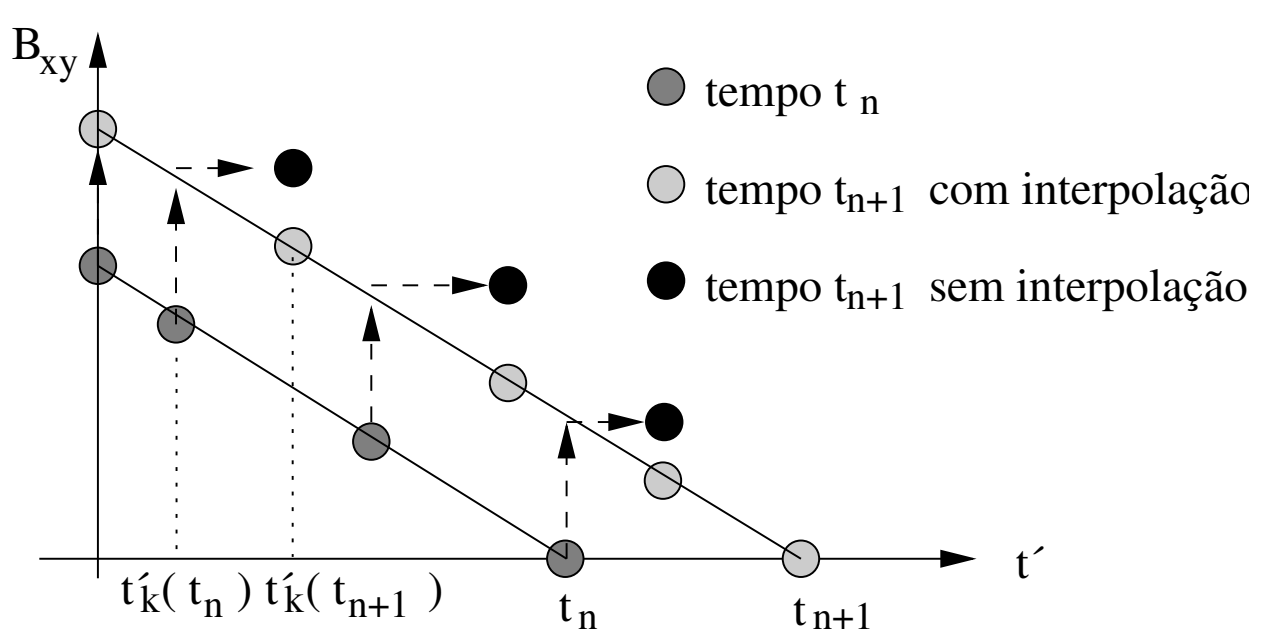

Figura 2.6: Cálculo do tensor de Finger nos tempos $t_{k}^{\prime}\left(t_{n+1}\right)$ sem interpolação

\subsubsection{Cálculo das tensões}

Uma vez obtido o campo de deformação $\mathbf{B}_{t^{\prime}\left(t_{n+1}\right)}\left(\mathbf{x}, t_{n+1}\right)$ a tensão pode então ser calculada. Escrevemos a integral em (2.8) na forma

$$
\boldsymbol{\tau}(\mathbf{x}, t)=\int_{-\infty}^{0} M\left(t-t^{\prime}\right) \mathbf{f}\left[\mathbf{B}_{t^{\prime}}(\mathbf{x}, t)\right] d t^{\prime}+\int_{0}^{t} M\left(t-t^{\prime}\right) \mathbf{f}\left[\mathbf{B}_{t^{\prime}}(\mathbf{x}, t)\right] d t^{\prime}
$$

onde, $\mathbf{f}\left[\mathbf{B}_{t^{\prime}}(\mathbf{x}, t)\right]=\frac{\alpha \mathbf{B}_{t^{\prime}}(\mathbf{x}, t)}{\alpha-3+\beta I_{1}+(1-\beta) I_{2}}$ para o modelo K-BKZ e simplesmente $\mathbf{f}\left[\mathbf{B}_{t^{\prime}}(\mathbf{x}, t)\right]=$ $\mathbf{B}_{t^{\prime}}(\mathbf{x}, t)$ para o modelo Maxwell . Admitimos que para $t^{\prime}<0$ tem-se $\mathbf{B}_{t^{\prime}}(\mathbf{x}, t)=$ $\mathbf{B}_{t_{0}^{\prime}}(\mathbf{x}, t)$. Com isso, dada a forma da função memória, a primeira integral em $(2.25)$ pode ser resolvida exatamente. Na segunda integral, no intervalo $[0, t]$, utilizando os 
pontos $t_{k}^{\prime}\left(t_{n+1}\right), i=0,1, \cdots, N$ (ver seção 2.3.1), podemos escrever

$$
\boldsymbol{\tau}(t)=\int_{-\infty}^{t_{0}^{\prime}} M\left(t-t^{\prime}\right) \mathbf{f}\left[\mathbf{B}_{t_{0}^{\prime}}\right] d t^{\prime}+\sum_{k=0}^{\frac{N-2}{2}}\left\{\int_{t_{2 k}^{\prime}}^{t_{2 k+2}^{\prime}} M\left(t-t^{\prime}\right) \mathbf{f}\left[\mathbf{B}_{t^{\prime}}(t)\right] d t^{\prime}\right\}
$$

Considerando $M\left(t-t^{\prime}\right)=\frac{a}{\lambda} e^{\frac{-\left(t-t^{\prime}\right)}{\lambda}}$, temos

$$
\boldsymbol{\tau}(t)=\int_{-\infty}^{t_{0}^{\prime}} \frac{a}{\lambda} e^{\frac{-\left(t-t^{\prime}\right)}{\lambda}} \mathbf{f}\left[\mathbf{B}_{t_{0}^{\prime}}\right] d t^{\prime}+\sum_{k=0}^{\frac{N-2}{2}}\left\{\int_{t_{2 k}^{\prime}}^{t_{2 k+2}^{\prime}} \frac{a}{\lambda} e^{\frac{-\left(t-t^{\prime}\right)}{\lambda}} \mathbf{f}\left[\mathbf{B}_{t^{\prime}}(t)\right] d t^{\prime}\right\}
$$

As integrais no somatório são resolvidas com uma fórmula de quadratura de segunda ordem, onde os coeficientes são encontrados usando o método dos coeficientes indeterminados [23]. Assim, para o cálculo da integral $\int_{t_{2 k}^{\prime}}^{t_{2 k+2}^{\prime}} \frac{a}{\lambda} e^{\frac{-\left(t-t^{\prime}\right)}{\lambda}} \mathbf{f}\left[\mathbf{B}_{t^{\prime}}(t)\right] d t^{\prime}$ usamos a fórmula de quadratura $I_{3}\left(t^{\prime}\right)=\sum_{j=1}^{3} A_{j} f\left(t_{j}^{\prime}\right)$, cujos coeficientes são obtidos a partir da solução do seguinte sistema linear :

$$
\begin{cases}I_{3}(1)=A_{1}+A_{2}+A_{3} & =b_{1} \\ I_{3}\left(t^{\prime}\right)=A_{1} \cdot t_{2 k}^{\prime}+A_{2} \cdot t_{2 k+1}^{\prime}+A_{3} \cdot t_{2 k+2}^{\prime}=b_{2} \\ I_{3}\left(t^{\prime 2}\right)=A_{1} \cdot t_{2 k}^{\prime 2}+A_{2} \cdot t_{2 k+1}^{\prime 2}+A_{3} \cdot t_{2 k+2}^{\prime 2}=b_{3}\end{cases}
$$

onde,

$$
\begin{aligned}
b_{1} & =\int_{t_{2 k}^{\prime}}^{t_{2 k+2}^{\prime}} \frac{1}{\lambda} e^{\frac{-\left(t-t^{\prime}\right)}{\lambda}} d t^{\prime}=e^{\frac{\left(t_{2 k+2}^{\prime}-t\right)}{\lambda}}-e^{\frac{\left(t_{2 k}^{\prime}-t\right)}{\lambda}}, \\
b_{2} & =\int_{t_{2 k}^{\prime}}^{t_{2 k+2}^{\prime}} \frac{1}{\lambda} e^{\frac{-\left(t-t^{\prime}\right)}{\lambda}} t^{\prime} d t^{\prime}=\left(t_{2 k+2}^{\prime}-\lambda\right) e^{\frac{\left(t_{2 k+2}^{\prime}-t\right)}{\lambda}}-\left(t_{2 k}^{\prime}-\lambda\right) e^{\frac{\left(t_{2 k}^{\prime}-t\right)}{\lambda}}, \\
b_{3} & =\int_{t_{2 k}^{\prime}}^{t_{2 k+2}^{\prime}} \frac{1}{\lambda} e^{\frac{-\left(t-t^{\prime}\right)}{\lambda}} t^{\prime 2} d t^{\prime}= \\
& =t_{2 k+2}^{\prime 2} e^{\frac{\left(t_{2 k+2}^{\prime}-t\right)}{\lambda}}-t_{2 k}^{\prime 2} e^{\frac{\left(t_{2 k}^{\prime}-t\right)}{\lambda}}-2 \lambda\left[\left(t_{2 k+2}^{\prime}-\lambda\right) e^{\frac{\left(t_{2 k+2}^{\prime}-t\right)}{\lambda}}-\left(t_{2 k}^{\prime}-\lambda\right) e^{\left.\frac{\left(t_{2 k}^{\prime}-t\right)}{\lambda}\right]}\right]
\end{aligned}
$$

Portanto a fórmula de quadratura usada para o cálculo das tensões tem como coefi- 
cientes:

$$
\begin{aligned}
& A_{3}=\frac{\left(b_{3}-t_{2 k}^{\prime 2} b_{1}\right)-\left(b_{2}-t_{2 k}^{\prime} b_{1}\right)\left(t_{2 k+1}^{\prime}+t_{2 k}^{\prime}\right)}{\left(t_{2 k}^{\prime}-t_{2 k+2}^{\prime}\right)\left(t_{2 k+1}^{\prime}-t_{2 k+2}^{\prime}\right)} \\
& A_{2}=\frac{\left(b_{2}-t_{2 k}^{\prime} b_{1}\right)-\left(t_{2 k+2}^{\prime}-t_{2 k}^{\prime}\right) A_{3}}{t_{2 k+1}^{\prime}-t_{2 k}^{\prime}} \\
& A_{1}=b_{1}-\left(A_{2}+A_{3}\right)
\end{aligned}
$$

Caso $N$ não seja par, usamos um método de primeira ordem no último intervalo. Fórmulas de quadratura de ordem superior poderiam ser utilizadas com essa mesma idéia mas não foram testadas até o momento. Isso reduziria o número de integrais que deveriam ser calculadas em cada passo de tempo.

\subsection{Validação do cálculo das tensões}

A seguir, vamos aplicar o método numérico para o cálculo da tensão, desenvolvido na seção 2.3, em cisalhamento simples e em elongação uniaxial e comparar os resultados com as respectivas soluções analíticas obtidas pelo modelo de Maxwell. Para escoamentos cisalhantes simples, as componentes $\tau^{x y}$ e $\tau^{y y}$ não serão calculadas porque as componentes do tensor de Finger são polinômios de grau $\leq 1$. Nesse caso, o método de Euler aproxima esses valores exatamente e portanto a fórmula de quadratura utilizada fornece um resultado exato. Para escoamentos elongacionais uniaxiais, o erro na componente $\tau^{y y}$ foi muito pequeno em comparação com o erro na componente $\tau^{x x}$ e por isso apenas os erros em $\tau^{x x}$ serão apresentados.

A seguir, vamos analisar os erros obtidos pelo método numérico em função das taxas de cisalhamento $\dot{\gamma}$ e de elongação $\dot{\varepsilon}$. Vamos também variar a quantidade de campos de deformação, $\mathbf{B}_{t_{i}^{\prime}}(t)$, relacionada com o número $\mathrm{N}$ de subintervalos de $[0, t]$.

De fato, para escoamentos cisalhantes simples, o vetor de velocidade e o tensor de Finger são dados por

$$
\left\{\begin{array}{l}
u=\dot{\gamma} y, \\
v=0, \\
w=0,
\end{array} \quad \text { e } \quad \mathbf{B}_{t^{\prime}}(t)=\left(\begin{array}{cccc}
1+\dot{\gamma}^{2}\left(t-t^{\prime}\right)^{2} & \dot{\gamma}\left(t-t^{\prime}\right) & 0 \\
\dot{\gamma}\left(t-t^{\prime}\right) & 1 & 0 \\
0 & 0 & 1
\end{array}\right)\right.
$$

onde $\dot{\gamma}$ é a taxa de cisalhamento. Para valores negativos de $t^{\prime}$ adota-se o valor em $t^{\prime}=0$. Neste caso a solução exata para as tensões pode ser obtida facilmente com o cálculo das 
integrais:

$$
\begin{aligned}
& \tau^{x x}(t)=\int_{-\infty}^{0} \frac{a}{\lambda} e^{\left(-\frac{t-t^{\prime}}{\lambda}\right)}\left(1+\dot{\gamma}^{2} t^{2}\right) d t^{\prime}+\int_{0}^{t} \frac{a}{\lambda} e^{\left(-\frac{t-t^{\prime}}{\lambda}\right)}\left(1+\dot{\gamma}^{2}\left(t-t^{\prime}\right)^{2}\right) d t^{\prime} \\
& \tau^{x y}(t)=\int_{-\infty}^{0} \frac{a}{\lambda} e^{\left(-\frac{t-t^{\prime}}{\lambda}\right)}(\dot{\gamma} t) d t^{\prime}+\int_{0}^{t} \frac{a}{\lambda} e^{\left(-\frac{t-t^{\prime}}{\lambda}\right)} \dot{\gamma}\left(t-t^{\prime}\right) d t^{\prime} \\
& \tau^{y y}(t)=\tau^{z z}(t)=\int_{-\infty}^{0} \frac{a}{\lambda} e^{\left(-\frac{t-t^{\prime}}{\lambda}\right)} d t^{\prime}+\int_{0}^{t} \frac{a}{\lambda} e^{\left(-\frac{t-t^{\prime}}{\lambda}\right)} d t^{\prime}
\end{aligned}
$$

obtendo

$$
\begin{aligned}
\tau^{x x}(t) & =a e^{\frac{-t}{\lambda}}\left(1+\dot{\gamma}^{2} t^{2}\right)+a\left(1-e^{\frac{-t}{\lambda}}\right)+a \dot{\gamma}^{2}\left[2 \lambda^{2}-e^{\frac{-t}{\lambda}}\left(t^{2}+2 \lambda t+2 \lambda^{2}\right)\right] \\
\tau^{x y}(t) & =a \dot{\gamma} \lambda\left(1-e^{\frac{-t}{\lambda}}\right) \\
\tau^{y y}(t) & =\tau^{z z}(t)=a
\end{aligned}
$$

Para escoamentos elongacionais uniaxiais temos

$$
\left\{\begin{aligned}
u & =\dot{\varepsilon} x, \\
v & =-\frac{1}{2} \dot{\varepsilon} y, \\
w & =-\frac{1}{2} \dot{\varepsilon} z,
\end{aligned} \quad \text { e } \quad \mathbf{B}_{t^{\prime}}(t)=\left(\begin{array}{ccc}
e^{2 \dot{\varepsilon}\left(t-t^{\prime}\right)} & 0 & 0 \\
0 & e^{-\dot{\varepsilon}\left(t-t^{\prime}\right)} & 0 \\
0 & 0 & e^{-\dot{\varepsilon}\left(t-t^{\prime}\right)}
\end{array}\right)\right. \text {. }
$$

Novamente, como no caso de cisalhamento simples, para valores negativos de $t^{\prime}$ adota-se o valor em $t^{\prime}=0$. As componentes da tensão são obtidas resolvendo as integrais

$$
\begin{gathered}
\tau^{x x}(t)=\int_{-\infty}^{0} \frac{a}{\lambda} e^{\left(-\frac{t-t^{\prime}}{\lambda}\right)} e^{2 \dot{\varepsilon} t} d t^{\prime}+\int_{0}^{t} \frac{a}{\lambda} e^{\left(-\frac{t-t^{\prime}}{\lambda}\right)} e^{2 \dot{\varepsilon}\left(t-t^{\prime}\right)} d t^{\prime} \\
\tau^{y y}(t)=\tau^{z z}(t)=\int_{-\infty}^{0} \frac{a}{\lambda} e^{\left(-\frac{t-t^{\prime}}{\lambda}\right)} e^{-\dot{\varepsilon} t} d t^{\prime}+\int_{0}^{t} \frac{a}{\lambda} e^{\left(-\frac{t-t^{\prime}}{\lambda}\right)} e^{-\dot{\varepsilon}\left(t-t^{\prime}\right)} d t^{\prime}
\end{gathered}
$$

obtendo

$$
\begin{gathered}
\tau^{x x}(t)=a e^{\left(\frac{-t(1-2 \lambda \dot{\varepsilon})}{\lambda}\right)}\left[1-\frac{1}{1-2 \lambda \dot{\varepsilon}}\right]+\frac{a}{1-2 \lambda \dot{\varepsilon}} \\
\tau^{y y}(t)=a e^{\left(-\frac{t(1+\lambda \dot{\varepsilon})}{\lambda}\right)}\left[1-\frac{1}{1+\lambda \dot{\varepsilon}}\right]+\frac{a}{1+\lambda \dot{\varepsilon}}
\end{gathered}
$$

A dificuldade numérica neste caso é maior porque $\tau^{x x}$ tende a crescer com o tempo e há uma singularidade para $\lambda \dot{\varepsilon}=\frac{1}{2}$. Para que $\tau^{x x}$ não cresça indefinidamente com o tempo, vamos usar valores de $\lambda$ e de $\dot{\varepsilon}$ para os quais tenhamos $\lambda \dot{\varepsilon}<\frac{1}{2}$. 
Introduzindo as velocidades para escoamentos cisalhantes simples e elongacionais uniaxiais nas equações $(2.21)-(2.23)$ obtemos

$$
\left\{\begin{array}{l}
\text { Caso cisalhante } \\
\left\{\begin{array} { l } 
{ \frac { \partial } { \partial t } B _ { t ^ { \prime } } ^ { y y } = 0 } \\
{ \frac { \partial } { \partial t } B _ { t ^ { \prime } } ^ { x y } = \dot { \gamma } B _ { t ^ { \prime } } ^ { y y } } \\
{ \frac { \partial } { \partial t } B _ { t ^ { \prime } } ^ { x x } = 2 \dot { \gamma } B _ { t ^ { \prime } } ^ { x y } }
\end{array} \quad \left\{\begin{array}{l}
\frac{\partial}{\partial t} B_{t^{\prime}}^{x x}=2 \dot{\varepsilon} B_{t^{\prime}}^{x x} \\
\frac{\partial}{\partial t} B_{t^{\prime}}^{y y}=-\dot{\varepsilon} B_{t^{\prime}}^{y y}
\end{array} .\right.\right.
\end{array}\right.
$$

A seguir, iremos determinar as componentes da tensão usando a metodologia descrita nas seções 2.3.1, 2.3.2 e 2.3.3 e comparar as soluções numéricas com as respectivas soluções analiticas. Os resultados são mostrados nas figuras 2.8, 2.9 e 2.10.

As figuras (2.8) e (2.9) mostram o erro relativo no cálculo da componente $\tau^{x x}$ para os escoamentos elongacional unixial e escoamento cisalhante simples, no tempo $t=10 \lambda$. Os dados utilizados foram $a=100 \mathrm{~Pa}$ e $\lambda=1 \mathrm{~s}$ e o passo de tempo no método de Euler foi $\delta \mathrm{t}=10^{-6} \mathrm{~s}$. Os resultados mostrados na figura 2.8 foram obtidos variando o valor de $N$. As taxas de cisalhamento e de elongação foram mantidas constantes e iguais a $\dot{\gamma}=1 s^{-1}$ e $\dot{\varepsilon}=0.3 s^{-1}$, respectivamente. Para o caso de escoamento cisalhante simples não houve variação no erro quando o valor de $N$ foi variado. De fato, a componente $B^{x x}$ do tensor de Finger é um polinômio de segunda ordem, o que faz com que as interpolações e a fórmula de quadratura sejam calculadas exatamente. O erro neste caso deve-se basicamente ao erro cometido na aproximação do tensor de Finger ao usar o método de Euler. Podemos observar na figura (2.8) que os resultados obtidos nesse trabalho, quando comparados com os obtidos por Hulsen et al. [22], apresentam erros muito menores para escoamentos cisalhantes simples, enquanto que para escoamentos elongacionais uniaxiais, os resultados apresentados por Hulsen et al. [22] são melhores.

De fato, para o escoamento elongacional uniaxial, a componente do tensor de Finger é dada por uma exponencial e os erros tendem a crescer com o tempo. Isso ocorre porque o comprimento dos intervalos de integração aumenta com o crescimento do tempo, o que afeta a precisão das fórmulas de interpolação e de quadratura. Esse problema é influenciado pela metodologia utilizada para discretizar o instante passado $t^{\prime}$. Dependendo dos parâmetros na função memória, a maioria dos tempos $t_{i}^{\prime}$ estarão concentrados em um intervalo $\left[t_{c}^{\prime}, t\right]$ (ver figura 2.7a) com espaçamentos $\delta t_{i}^{\prime}=t_{i+1}^{\prime}-t_{i}^{\prime}<<1$ enquanto que os restantes $t_{i}^{\prime}$ no intervalo $\left[0, t_{c}^{\prime}\right]$ têm espaçamento $\delta t_{i}^{\prime}=t_{i+1}^{\prime}-t_{i}^{\prime}>>1$. Esse fato, pode 
ocasionar grandes erros no cálculo das tensões em escoamentos elongacionais uniaxiais.

Por outro lado, se fizermos uma discretização utilizando pontos equidistantes com $\delta t^{\prime}=\frac{t}{N}$ (ver figura 2.7b) então, para tempos não muito grandes, os pontos $t_{i}^{\prime}$ ficam melhores distribuídos no intervalo $[0, t]$, de modo que a precisão das fórmulas de interpolação de quadratura são maiores. A figura 2.10 mostra esse efeito, onde são também apresentados para comparação os resultados de Hulsen et al. [22]. Como era de se esperar essa mudança não afeta o cálculo no caso de escoamento cisalhante. Em comparação com os resultados de Hulsen et al. [22], o erro em $\tau^{x x}$ para escoamento elongacional uniaxial apresenta a mesma ordem de grandeza. Existem muitas maneiras de fazer a discretização do intervalo $[0, t]$. Os resultados apresentados nesta tese foram obtidos usando a discretização da seção 2.3.1. Caso os escoamentos tenham uma forte elongação deve-se tomar os cuidados necessários na discretização para evitar erros maiores.

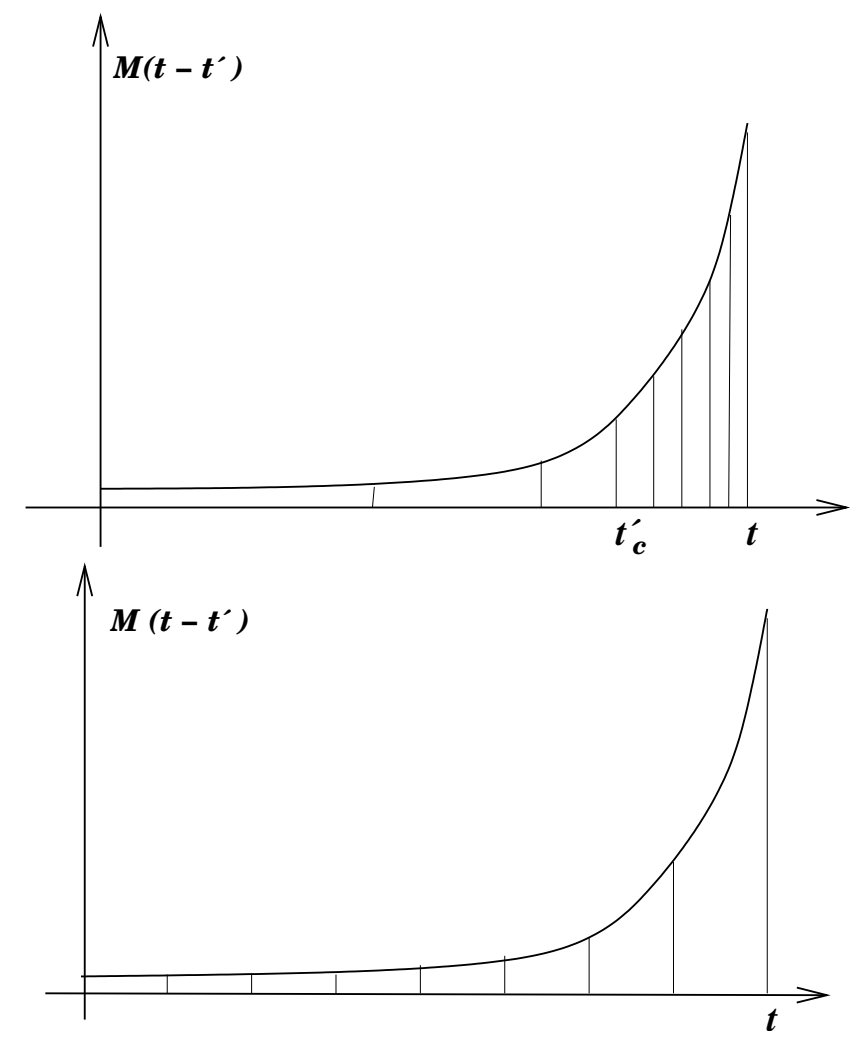

Figura 2.7: Cálculo dos pontos de integração $t_{i}^{\prime}$ : discretização do intervalo $[0, t]$. a) discretização conforme metodologia descrita na seção [2.3.1, b) discretização utilizando pontos igualmente espaçados. 


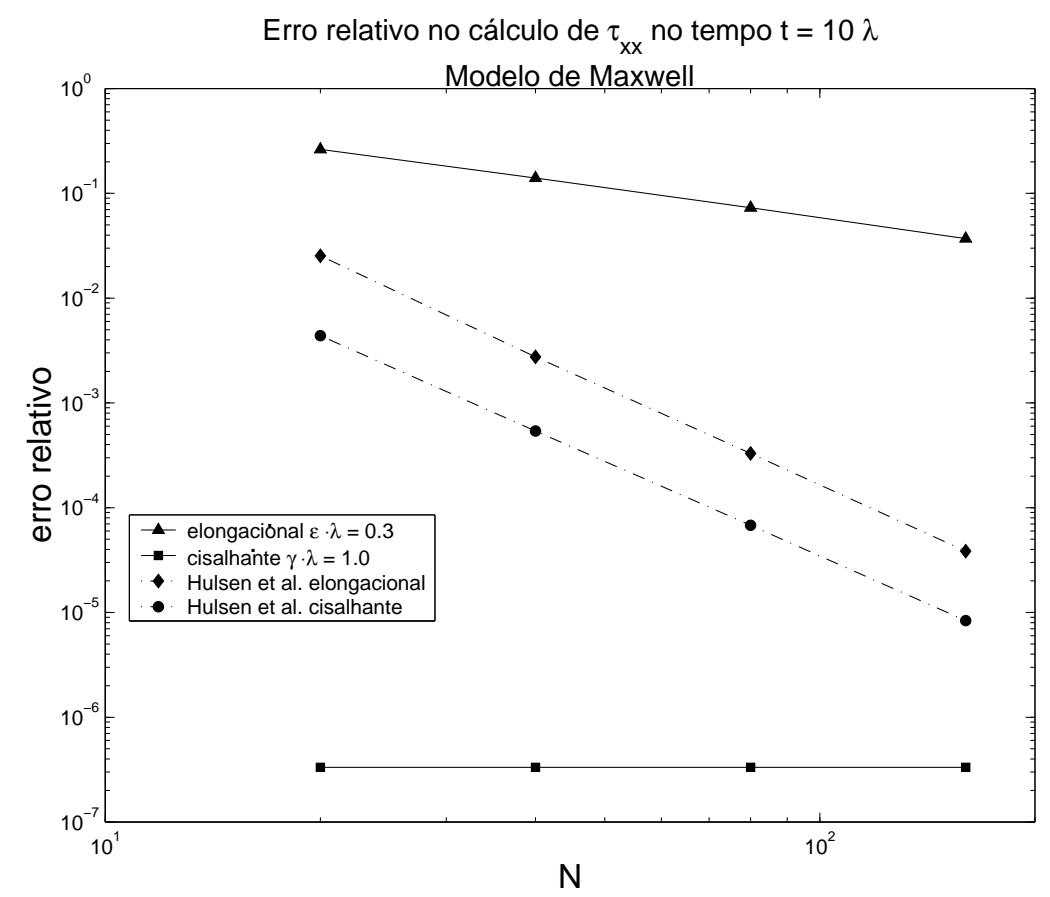

Figura 2.8: Erro relativo no cálculo de $\tau^{x x}$ no tempo $t=10 \lambda$ em função de $N$. As taxas de elongação e de cisalhamento estão fixas e são iguais a $\dot{\gamma}=1 \mathrm{~s}^{-1}$ e $\dot{\varepsilon}=0.3 \mathrm{~s}^{-1}$.

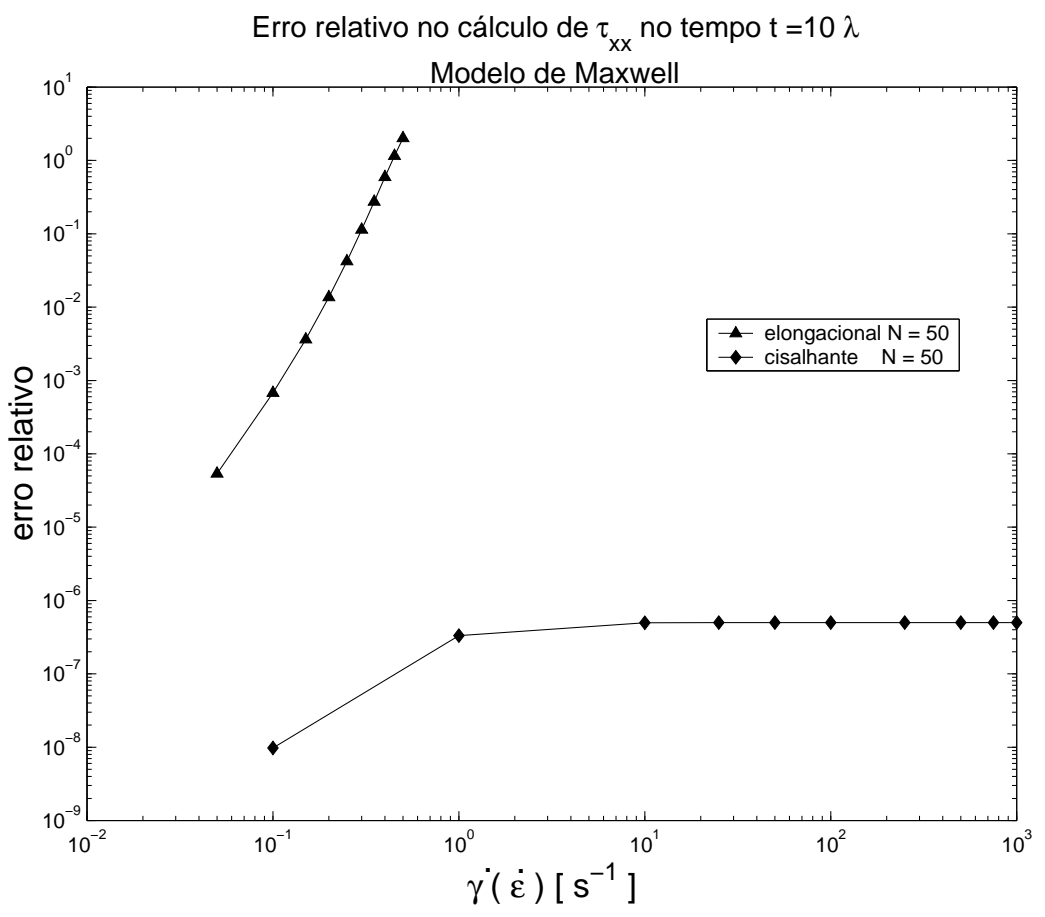

Figura 2.9: Erro relativo no cálculo da componente $\tau^{x x}$ para escoamentos elongacional e cisalhante em função das taxas $\dot{\gamma}$ e $\dot{\varepsilon} . \quad N=50$ é mantido fixo. 

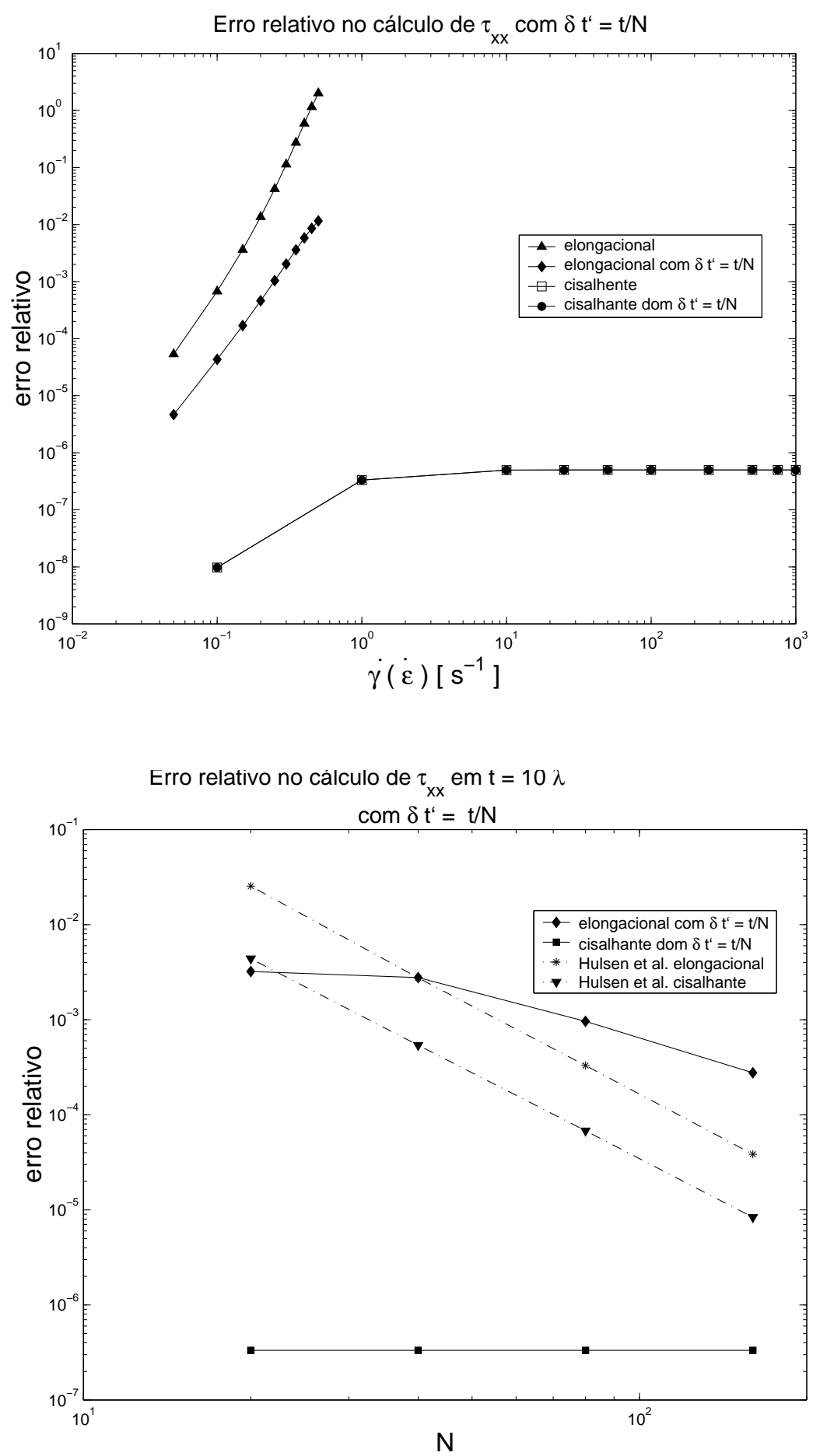

Figura 2.10: Comparação entre o erro para duas formas de discretização do instante passado $t^{\prime}$. Em uma outra discretização foi considerada a situação simples $\delta t^{\prime}=\frac{t}{N}$. 


\section{Capítulo 3}

\section{Extensão de GENSMAC para}

\section{modelos integrais de Maxwell e}

\section{K-BKZ}

Este capítulo apresenta um método numérico para simular escoamentos viscoelásticos bidimensionais governados pelos modelos reológicos integrais de Maxwell e K-BKZ. O método apresentado é uma extensão do método GENSMAC (Generalized-Simplified-Marker-andCell ), introduzido por Tomé e McKee[61], que utiliza o método de diferenças finitas numa malha deslocada e utiliza partículas virtuais para a visualização e localização da superfície livre do fluido. A metodologia apresentada pode ser utilizada para simular escoamentos confinados e escoamentos com superfície livre.

\subsection{Idéia Básica do método GENSMAC}

O método GENSMAC faz parte de um grupo de métodos numéricos conhecidos como métodos de projeção, dos quais também estão o métodos MAC [21] e SMAC [4], sendo o método GENSMAC uma generalização deste último. Nos métodos de projeção, a solução para a equação de Navier-Stokes é obtida primeiramente avançando a velocidade no tempo sem a preocupação da restrição de divergente nulo, dada pela equação da continuidade, e então recuperando a propriedade de campo selenoidal de velocidade, $(\nabla \cdot \mathbf{v}=0)$, por meio de um operador de projeção. Este operador projeção é obtido através do teorema de decomposição de Helmholtz-Hodge [12], onde a velocidade é decomposta em um vetor 
selenoidal, v, e o gradiente de um potencial, $\nabla \psi$. Esta decomposição é escrita como [25]

$$
\widetilde{\mathbf{v}}=\mathbf{v}+\nabla \psi
$$

onde $\widetilde{\mathbf{v}}$ é calculado utilizando a equação de Navier-Stokes. Tomando o divergente de (3.1) e impondo que $\mathbf{v}$ seja solenoidal, obtemos uma equação elíptica para $\psi$, como segue:

$$
\nabla \cdot \widetilde{\mathbf{v}}=\nabla \cdot \mathbf{v}+\nabla \cdot \nabla \psi \Longrightarrow \nabla \cdot \widetilde{\mathbf{v}}=\nabla^{2} \psi
$$

Um vez que a solução $\psi$ é obtida, v segue da correção

$$
\mathbf{v}=\widetilde{\mathbf{v}}-\nabla \psi
$$

\subsection{Equações governantes}

As equações governantes para escoamentos isotérmicos incompressíveis são a equação de conservação de massa

$$
\nabla \cdot \mathbf{v}=0
$$

e a equação de conservação de quantidade de movimento

$$
\rho\left[\frac{\partial \mathbf{v}}{\partial t}+\nabla \cdot(\mathbf{v} \mathbf{v})\right]=-\nabla p+\nabla \cdot \boldsymbol{\tau}+\rho \mathbf{g}
$$

onde $\rho$ é a densidade, $\mathbf{v}$ é o vetor velocidade, $p$ é a pressão, $g$ é a gravidade e $\boldsymbol{\tau}$ é o tensor tensão, determinado pela equação constitutiva do fluido. Neste trabalho estaremos interessados em escoamentos de fluidos viscoelásticos definidos pelos modelos constitutivos na forma integral de Maxwell e K-BKZ. O modelo K-BKZ, na forma fatorada, é dado por

$$
\boldsymbol{\tau}(t)=\int_{-\infty}^{t} M\left(t-t^{\prime}\right) H\left(I_{1}, I_{2}\right) \mathbf{B}_{t^{\prime}}(t) d t^{\prime}
$$

onde $M\left(t-t^{\prime}\right)$ é a função memória, $H\left(I_{1}, I_{2}\right)$ é a função amortecimento e $\mathbf{B}_{t^{\prime}}(t)$ é o tensor de Finger, que mede a deformação de um elemento de fluido que está na posição $\mathbf{x}$ no instante presente $t$, com relação a sua posição num instante anterior $t^{\prime} . I_{1}$ e $I_{2}$ são o primeiro e segundo invariantes de $\mathbf{B}_{t^{\prime}}(t)$, respectivamente, dados por $I_{1}=\operatorname{tr}(\mathbf{B})$ e $I_{2}=\frac{1}{2}\left[I_{1}^{2}-\operatorname{tr}\left(\mathbf{B}^{2}\right)\right]$. No que se segue usaremos o modelo de Papanastasiou, Scriven e Macosko (PSM), em que [47]:

$$
M\left(t-t^{\prime}\right)=\sum_{m} \frac{a_{m}}{\lambda_{m}} e^{-\frac{t-t^{\prime}}{\lambda_{m}}}
$$


e

$$
H\left(I_{1}, I_{2}\right)=\frac{\alpha}{\alpha+\beta I_{1}+(1-\beta) I_{2}} .
$$

As constantes $a_{m}, \lambda_{m}, \alpha$ e $\beta$ são parâmetros que dependem do material, dados a priori. O modelo de Maxwell é obtido fazendo $H\left(I_{1}, I_{2}\right) \equiv 1$ na equação (3.4).

Para resolver as equações (3.2)-(3.4), escrevemos o tensor tensão na forma

$$
\boldsymbol{\tau}=\mathbf{S}+\eta_{0} \dot{\gamma}
$$

onde $\dot{\gamma}=\nabla \mathbf{v}+(\nabla \mathbf{v})^{t}$ e $\eta_{0}$ é a viscosidade a baixas taxas de cisalhamento.

Substituindo (3.7) na equação (3.3) obtemos

$$
\rho\left[\frac{\partial \mathbf{v}}{\partial t}+\nabla \cdot(\mathbf{v} \mathbf{v})\right]=-\nabla p+\eta_{0} \nabla^{2} \mathbf{v}+\nabla \cdot \mathbf{S}+\rho \mathbf{g} .
$$

Para resolver as equações (3.2), (3.8), (3.4) e (3.7), introduzimos a adimensionalização $\overline{\mathbf{v}}=\frac{\mathbf{v}}{U}, \overline{\mathbf{x}}=\frac{\mathbf{x}}{L}, \bar{t}=\frac{U}{L} t, \overline{\mathbf{g}}=\frac{\mathbf{g}}{g}, \bar{p}=\frac{p}{\rho_{0} U^{2}}, \overline{\mathbf{S}}=\frac{\mathbf{S}}{\rho_{0} U^{2}}, \bar{\eta}=\frac{\eta}{\eta_{0}}, \bar{\rho}=\frac{\rho}{\rho_{0}}, \bar{\lambda}_{m}=\frac{\lambda_{m}}{\lambda_{r e f}}, \bar{a}_{m}=\frac{a_{m}}{\rho_{0} U^{2}}$

onde $L, U, g, \eta_{0}, \rho_{0}, \lambda_{r e f}$ são valores característicos de comprimento, velocidade, gravidade, viscosidade, densidade e tempo de relaxação, respectivamente. Após introduzir essas variáveis nas equações (3.2), (3.8), (3.4) e (3.7) e omitir as barras, obtemos as equações na forma adimensional

$$
\begin{aligned}
\nabla \cdot \mathbf{v} & =0 \\
\frac{\partial \mathbf{v}}{\partial t}+\nabla \cdot(\mathbf{v} \mathbf{v}) & =-\nabla p+\frac{1}{R e} \nabla^{2} \mathbf{v}+\nabla \cdot \mathbf{S}+\frac{1}{F r^{2}} \mathbf{g} \\
\boldsymbol{\tau}(t) & =\int_{-\infty}^{t} \sum_{m} \frac{a_{m}}{\lambda_{m} W e} e^{-\frac{t-t^{\prime}}{\lambda m W e}} \frac{\alpha}{\alpha-3+\beta I_{1}+(1-\beta) I_{2}} \mathbf{B}_{t^{\prime}}(t) d t^{\prime} \\
\mathbf{S} & =\boldsymbol{\tau}-\frac{1}{R e} \dot{\gamma}
\end{aligned}
$$

onde $R e=\frac{\rho_{0} U L}{\eta_{0}}$ é o número de Reynolds, $W e=\frac{\lambda_{r e f} U}{L}$ é o número de Weissenberg e $F r=\frac{U}{\sqrt{L g}}$ é o número de Froude.

Neste trabalho vamos considerar escoamentos bidimensionais, com $\mathbf{v}=(u, v)$ e $\mathbf{x}=$ $(x, y)$. Neste caso, utilizando coordenadas cartesianas, as equações (3.9)-(3.12) são escritas como

$$
\frac{\partial u}{\partial x}+\frac{\partial v}{\partial y}=0
$$




$$
\begin{gathered}
\frac{\partial u}{\partial t}+\frac{\partial\left(u^{2}\right)}{\partial x}+\frac{\partial(u v)}{\partial y}=-\frac{\partial p}{\partial x}+\frac{1}{R e}\left[\frac{\partial^{2} u}{\partial x^{2}}+\frac{\partial^{2} u}{\partial y^{2}}\right]+\frac{\partial S^{x x}}{\partial x}+\frac{\partial S^{x y}}{\partial y}+\frac{1}{F r^{2}} g_{x} \\
\frac{\partial v}{\partial t}+\frac{\partial(u v)}{\partial x}+\frac{\partial\left(v^{2}\right)}{\partial y}=-\frac{\partial p}{\partial y}+\frac{1}{R e}\left[\frac{\partial^{2} v}{\partial x^{2}}+\frac{\partial^{2} v}{\partial y^{2}}\right]+\frac{\partial S^{x y}}{\partial x}+\frac{\partial S^{y y}}{\partial y}+\frac{1}{F r^{2}} g_{y} \\
\tau^{x x}=\int_{-\infty}^{t} \sum_{m} \frac{a_{m}}{W e \lambda_{m}} e^{-\frac{t-t^{\prime}}{W e \lambda_{m}}} \frac{\alpha}{\alpha-3+\beta I_{1}+(1-\beta) I_{2}} B_{t^{\prime}}^{x x}(t) d t^{\prime} \\
\tau^{x y}=\int_{-\infty}^{t} \sum_{m} \frac{a_{m}}{W e \lambda_{m}} e^{-\frac{t-t^{\prime}}{W e \lambda_{m}}} \frac{\alpha}{\alpha-3+\beta I_{1}+(1-\beta) I_{2}} B_{t^{\prime}}^{x y}(t) d t^{\prime} \\
\int_{-\infty}^{t} \sum_{m} \frac{a_{m}}{W e \lambda_{m}} e^{-\frac{t-t^{\prime}}{W e \lambda_{m}}} \frac{\alpha}{\alpha-3+\beta I_{1}+(1-\beta) I_{2}} B_{t^{\prime}}^{y y}(t) d t^{\prime} \\
S^{x x}=\tau^{x x}-\frac{2}{R e}\left(\frac{\partial u}{\partial x}\right) \\
S^{x y}=\tau^{x y}-\frac{1}{R e}\left(\frac{\partial u}{\partial y}+\frac{\partial v}{\partial x}\right) \\
S^{y y}-\frac{2}{R e}\left(\frac{\partial v}{\partial y}\right)
\end{gathered}
$$

onde $B_{t^{\prime}}^{x x}(t), B_{t^{\prime}}^{x y}(t)$ e $B_{t^{\prime}}^{y y}(t)$ são dados pelas equações (2.21)-(2.23).

Portanto, para simular escoamentos viscoelásticos governados pelos modelos de Maxwell e K-BKZ, precisamos resolver as equações (3.13)-(3.21) sujeitas a condições iniciais e de contorno.

\subsection{Condições de contorno}

Fronteira rígida: é imposta a condição de não escorregamento (no-slip). Neste caso, as componentes normal e tangencial do vetor velocidade são nulas.

Injetores: Impomos um perfil parabólico newtoniano para a velocidade ou um perfil constante.

Ejetores: Impomos condição de Neumann homogênea, ou seja,

$$
\frac{\partial u_{n}}{\partial n}=0 \quad \text { e } \frac{\partial u_{m}}{\partial n}=0
$$

onde $n$ e $m$ designam as direções normal e tangencial aos ejetores, respectivamente. 


\subsection{Cálculo do tensor de Finger nos contornos}

Ao calcular as componentes do tensor de Finger usando as equações (2.21)-(2.23) usamos um método upwind de alta ordem para aproximar os termos convectivos. Neste trabalho usamos o método CUBISTA (Convergent and Universally Bounded Interpolation Scheme for the Treatment of Advection), desenvolvido por Alves et al. [2]. Este método necessita que os valores das variáveis sejam conhecidas em até dois nós vizinhos. Para cálculos em pontos próximos aos contornos há a necessidade de se conhecer o valor do tensor de Finger nas células de contorno.

\subsubsection{Cálculo do tensor de Finger nos contornos rígidos}

\section{Contornos rígidos paralelos ao eixo $x$}

Nesses contornos temos $u=v=0 \Longrightarrow \frac{\partial u}{\partial x}=\frac{\partial v}{\partial x}=\frac{\partial v}{\partial y}=0$ (pela equação da continuidade). Neste caso, as equações (2.21)-(2.23) podem ser escritas na forma

$$
\frac{\partial}{\partial t} B_{t^{\prime}}^{x x}=2 \frac{\partial u}{\partial y} B_{t^{\prime}}^{x y}, \quad \frac{\partial}{\partial t} B_{t^{\prime}}^{x y}=\frac{\partial u}{\partial y} B_{t^{\prime}}^{y y}, \quad \frac{\partial}{\partial t} B_{t^{\prime}}^{y y}=0 .
$$

As equações (3.22) podem ser resolvidas facilmente usando uma aproximação por diferenças finitas. O cálculo do tensor de Finger em fronteiras paralelas ao eixo y é semelhante.

\section{Contornos rígidos paralelos ao eixo $y$}

Neste caso a condição "no-slip" e a equação da continuidade implicam

$$
u=v=\frac{\partial u}{\partial x}=\frac{\partial v}{\partial x}=\frac{\partial v}{\partial y}=0
$$

e com isso as equações $(2.21)$ - (2.23) se reduzem a

$$
\frac{\partial}{\partial t} B_{t^{\prime}}^{x x}=0 \quad \frac{\partial}{\partial t} B_{t^{\prime}}^{x y}=\frac{\partial v}{\partial x} B_{t^{\prime}}^{x x} \quad \frac{\partial}{\partial t} B_{t^{\prime}}^{y y}=2 \frac{\partial v}{\partial x} B_{t^{\prime}}^{x y} .
$$

\subsubsection{Cálculo do tensor de Finger nos injetores}

Nos injetores consideramos um escoamento cisalhante totalmente desenvolvido. Por exemplo, se tivermos injetores paralelos ao eixo y o tensor de Finger será dado por [8]

$$
B^{x x}=\left(\frac{\partial u}{\partial y}\right)^{2}\left(t-t^{\prime}\right)^{2}+1 ; \quad B^{x y}=\left(\frac{\partial u}{\partial y}\right)\left(t-t^{\prime}\right) ; \quad B^{y y}=1
$$




\subsubsection{Cálculo do tensor de Finger nos ejetores}

Nos ejetores assumimos válida a condição homogênea de Neumann para o tensor de Finger. Para ejetores paralelos ao eixo $y$ temos

$$
\frac{\partial B^{x x}}{\partial y}=\frac{\partial B^{x y}}{\partial y}=\frac{\partial B^{y y}}{\partial y}=0
$$

\subsection{Condições de superfície livre}

Vamos considerar escoamentos de fluidos viscosos numa atmosfera passiva e vamos supor que os efeitos de tensão superficial são pequenos e podem ser ignorados. Neste caso, as condições de tensão na superfície livre podem ser escritas como [6]

$$
\mathbf{n} \cdot(\boldsymbol{\sigma} \cdot \mathbf{n})=0 \text { e } \mathbf{m} \cdot(\boldsymbol{\sigma} \cdot \mathbf{n})=0
$$

onde $\boldsymbol{\sigma}=p \mathbf{I}+\boldsymbol{\tau}$ é o tensor tensão total, com $\boldsymbol{\tau}$ dado pela equação constitutiva do fluido.

Da equação $\mathbf{n} \cdot \boldsymbol{\sigma} \cdot \mathbf{n}=0$ segue que

$$
p=n_{x}^{2} \tau_{x x}+2 n_{x} n_{y} \tau_{x y}+n_{y}^{2} \tau_{y y}
$$

e de $\mathbf{m} \cdot \boldsymbol{\sigma} \cdot \mathbf{n}=0$ vem

$$
m_{x} n_{x} \tau_{x x}+\left(m_{x} n_{y}+m_{y} n_{x}\right) \tau_{x y}+m_{y} n_{y} \tau_{y y}=0
$$

Tomando $m_{x}=-n_{y}$ e $m_{y}=n_{x}$ obtemos

$$
n_{x} n_{y}\left(\tau_{y y}-\tau_{x x}\right)+\left(n_{x}^{2}-n_{y}^{2}\right) \tau_{x y}=0
$$

Considerando $\boldsymbol{\tau}=\mathbf{S}+\eta_{0} \dot{\gamma}$ temos

1. $\operatorname{de} \mathbf{n} \cdot \mathbf{T} \cdot \mathbf{n}=0$

$$
\begin{gathered}
p=n_{x}^{2}\left[S_{x x}+2 \eta_{0} \frac{\partial u}{\partial x}\right]+2 n_{x} n_{y}\left[S_{x y}+\eta_{0}\left(\frac{\partial u}{\partial y}+\frac{\partial v}{\partial x}\right)\right]+n_{y}^{2}\left[S_{y y}+2 \eta_{0} \frac{\partial v}{\partial y}\right] \\
p=2 \eta_{0}\left[n_{x}^{2} \frac{\partial u}{\partial x}+n_{x} n_{y}\left(\frac{\partial u}{\partial y}+\frac{\partial v}{\partial x}\right)+n_{y}^{2} \frac{\partial v}{\partial y}\right]+n_{x}^{2} S_{x x}+2 n_{x} n_{y} S_{x y}+n_{y}^{2} S_{y y}
\end{gathered}
$$

que na forma adimensional fica

$$
p=\frac{2}{R e}\left[n_{x}^{2} \frac{\partial u}{\partial x}+n_{x} n_{y}\left(\frac{\partial u}{\partial y}+\frac{\partial v}{\partial x}\right)+n_{y}^{2} \frac{\partial v}{\partial y}\right]+n_{x}^{2} S_{x x}+2 n_{x} n_{y} S_{x y}+n_{y}^{2} S_{y y}
$$


2. $\quad$ De $\mathbf{m} \cdot \mathbf{T} \cdot \mathbf{n}=0$

$$
\begin{aligned}
& m_{x} n_{x}\left(2 \eta_{0} \frac{\partial u}{\partial x}\right)+\left(m_{x} n_{y}+m_{y} n_{x}\right) \eta_{0}\left(\frac{\partial u}{\partial y}+\frac{\partial v}{\partial x}\right)+m_{y} n_{y}\left(2 \eta_{0} \frac{\partial v}{\partial y}\right) \\
& +m_{x} n_{x} S_{x x}+\left(m_{x} n_{y}+m_{y} n_{x}\right) S_{x y}+m_{y} n_{y} S_{y y}=0
\end{aligned}
$$

que na forma adimensional fica

$$
\begin{aligned}
& \frac{2}{R e}\left[m_{x} n_{x} \frac{\partial u}{\partial x}+\frac{1}{2}\left(m_{x} n_{y}+m_{y} n_{x}\right)\left(\frac{\partial u}{\partial y}+\frac{\partial v}{\partial x}\right)+m_{y} n_{y} \frac{\partial v}{\partial y}\right] \\
& +m_{x} n_{x} S_{x x}+\left(m_{x} n_{y}+m_{y} n_{x}\right) S_{x y}+m_{y} n_{y} S_{y y}=0
\end{aligned}
$$

\subsection{Método de Solução}

Para resolver as equações (3.13)-(3.21), vamos utilizar o seguinte algoritmo que é uma extensão do método GENSMAC para escoamentos de fluidos viscoelásticos.

Dado o campo de velocidade e o tensor extra-tensão no tempo $t_{n}$, com as respectivas condições de contorno, o cálculo da velocidade $\mathbf{v}$, pressão $p$ e o tensor tensão $\boldsymbol{\tau}$ no tempo $t_{n+1}=t_{n}+\delta t$, onde $\delta t>0$ dado, são obtidos como segue:

Passo 1 Seja $\widetilde{p}\left(\mathbf{x}, t_{n}\right)$ um campo de pressão que satisfaz a condição correta para a pressão na superfície livre. Esse campo de pressão é calculado a partir da condição para tensão na superfície livre $\mathbf{n} \cdot(\boldsymbol{\sigma} \cdot \mathbf{n})=0$ onde $\boldsymbol{\sigma}=-\widetilde{p} \mathbf{I}+\boldsymbol{\tau}$. Detalhes sobre o cálculo de $\widetilde{p}$ serão dados na seção 3.7 .4 .

Passo 2 Calcular o campo de velocidade intermediário, $\tilde{\mathbf{v}}\left(\mathbf{x}, t_{n+1}\right)$, usando as equações

$$
\begin{aligned}
& \frac{\partial \tilde{u}}{\partial t}=-\frac{\partial \widetilde{p}}{\partial x}-\frac{\partial\left(u^{2}\right)}{\partial x}-\frac{\partial(v u)}{\partial y}+\frac{1}{R e}\left(\frac{\partial^{2} u}{\partial x^{2}}+\frac{\partial^{2} u}{\partial y^{2}}\right)+\frac{\partial S^{x x}}{\partial x}+\frac{\partial S^{x y}}{\partial y}+\frac{1}{F r^{2}} g_{x} \\
& \frac{\partial \tilde{v}}{\partial t}=-\frac{\partial \widetilde{p}}{\partial y}-\frac{\partial(u v)}{\partial x}-\frac{\partial\left(v^{2}\right)}{\partial y}+\frac{1}{R e}\left(\frac{\partial^{2} v}{\partial x^{2}}+\frac{\partial^{2} v}{\partial y^{2}}\right)+\frac{\partial S^{x y}}{\partial x}+\frac{\partial S^{y y}}{\partial y}+\frac{1}{F r^{2}} g_{y}
\end{aligned}
$$

Passo 3 Resolver a equação de Poisson:

$$
\nabla^{2} \psi\left(\mathbf{x}, t_{n+1}\right)=\nabla \cdot \tilde{\mathbf{v}}\left(\mathbf{x}, t_{n+1}\right)
$$

com a condição $\frac{\partial \psi}{\partial n}=0$ nas fronteiras rígidas e injetores; $\psi=0$ na superfície livre e ejetores. 
Passo 4 Calcular o campo de velocidade

$$
\mathbf{v}\left(\mathbf{x}, t_{n+1}\right)=\tilde{\mathbf{v}}\left(\mathbf{x}, t_{n+1}\right)-\nabla \psi\left(\mathbf{x}, t_{n+1}\right)
$$

Passo 5 Calcular a pressão

$$
p\left(\mathbf{x}, t_{n+1}\right)=\widetilde{p}\left(\mathbf{x}, t_{n}\right)+\frac{\psi\left(\mathbf{x}, t_{n+1}\right)}{\delta t}
$$

Passo 6 Calcular o tensor tensão $\boldsymbol{\tau}\left(\mathbf{x}, t_{n+1}\right)$ seguindo os seguintes passos:

6.1 Calcular os nós de integração $t_{i}^{\prime}, i=1, \cdots, N$, usando o procedimento descrito na seção 2.3.1.

6.2 Calcular as componentes do tensor de Finger nos contornos rígidos, nas entradas e nas saídas de fluido, de acordo com as equações apresentadas na seção 3.4 .

6.3 Calcular as componentes do tensor de Finger $\mathbf{B}_{t^{\prime}}\left(\mathbf{x}, t_{n+1}\right)$ conforme procedimento apresentado na seção 2.3.2, utilizando as equações (2.21)-(2.23).

6.4 Calcular as componentes do tensor tensão $\boldsymbol{\tau}\left(\mathbf{x}, t_{n+1}\right)$ usando o método descrito na seção 2.3.3, utilizando as equações (3.16) $-(3.18)$

Step 7 Calcular as componentes do tensor $\mathbf{S}$ por meio das equações (3.19) $-(3.21)$.

Step 8 Atualizar as posições das partículas marcadoras : O último passo no cálculo é mover as partículas marcadoras para suas novas posições. Isso é feito resolvendo

$$
\frac{d x}{d t}=u, \quad \frac{d y}{d t}=v
$$

para cada partícula. A superfície do fluido é definida por uma lista ordenada contendo essas partículas marcadoras, e a visualização da superfície livre é obtida simplesmente unindo esses pontos por segmentos de reta.

\subsection{Aproximação pelo método de diferenças finitas}

Para resolver as equações do algoritmo apresentado na seção 3.6 vamos utilizar o método de diferenças finitas sobre uma malha deslocada com espaçamento $\delta x$ e $\delta y$. Nesta malha, as velocidades são armazenadas no centro das faces das células enquanto que a pressão, 
o tensor tensão e o tensor de Finger são armazenados no centro das células (ver figura $3.7 \mathrm{a})$.

Neste trabalho vamos considerar escoamentos transientes com superfícies livres em movimento. Nesse caso, é necessário fazer uma classificação das células da malha para identificar se as mesmas contém ou não fluido, se estão no contorno rígido ou são células da superfície livre. Para tanto, adota-se a seguinte classificação:

- Células Vazias (E): São células que não contém fluido;

- Células Cheias (F): São células cheias de fluido e não possuem nenhuma face em contato com células Vazias;

- Células de Superfície (S): São células que contém fluido e possuem pelo menos uma face em contato com células Vazias;

- Células de Contorno (B): São células que definem o contorno rígido. Nessas células a condição de não escorregamento é imposta;

- Células 'Inflow' (I): São células que definem a entrada de fluido;

- Células 'Outflow' (O): São células que definem a saída de fluido.

A figura 3.7b mostra esses tipos de células em um dado instante.

a)

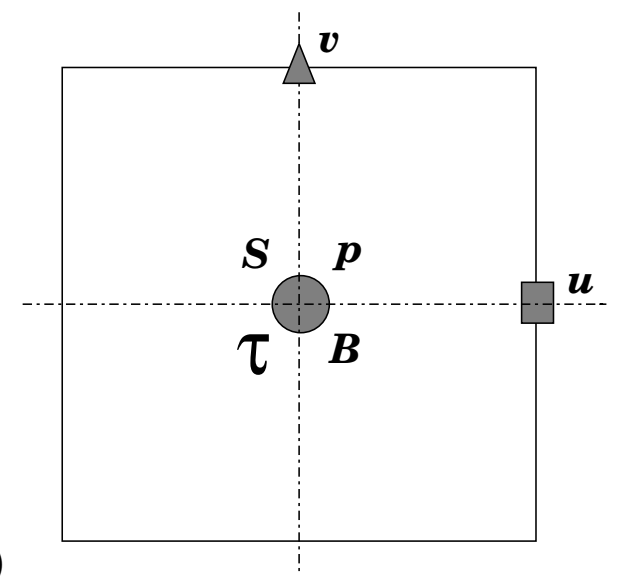

b)

\begin{tabular}{|c|c|c|c|c|c|c|c|c|c|c|c|c|c|}
\hline & & & & & \multirow{2}{*}{$\frac{B}{E}$} & \multirow{2}{*}{$\frac{1}{s}$} & \multirow{2}{*}{$\begin{array}{l}1 \\
\mathrm{~s}\end{array}$} & \multirow{2}{*}{$\frac{B}{E}$} & & & & & \\
\hline B & & & & & & & & & & & & & B \\
\hline B & & & & & $E$ & $\mathrm{~s}$ & $\mathbf{s}$ & $E$ & & & & & B \\
\hline B & & & & & $E$ & $\mathrm{~s}$ & $\mathbf{S}$ & E & & & & & B \\
\hline B & & & & & $E$ & $\mathbf{S}$ & S & $E$ & & & & & B \\
\hline B & & & & & $E$ & S & S & $E$ & & & & & B \\
\hline B & $E$ & E & & & $E$ & $\mathbf{S}$ & $\mathbf{S}$ & E & & & $E$ & E & B \\
\hline B & s & S & $E$ & & $E$ & $\mathbf{S}$ & S & E & & E & $\mathbf{s}$ & $\mathrm{s}$ & B \\
\hline B & $\mathrm{F}$ & $\mathbf{F}$ & 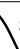 & $E$ & $E$ & s & $\mathrm{S}$ & $E$ & $\mathrm{E}$ & s/ & $\mathbf{F}$ & & B \\
\hline B & $\mathbf{F}$ & $\mathbf{F}$ & $F$ & S & $S$ & $\mathbf{F}$ & $\mathbf{F}$ & $s$ & s & $\mathrm{F}$ & $F$ & $\mathbf{F}$ & B \\
\hline B & $\mathbf{F}$ & $\mathbf{F}$ & $F$ & $F$ & $F$ & $\mathbf{F}$ & $F$ & $F$ & $F$ & $\mathbf{F}$ & $F$ & $\mathbf{F}$ & B \\
\hline B & $\mathbf{F}$ & $\mathbf{F}$ & $F$ & $F$ & $F$ & $\mathbf{F}$ & $\mathbf{F}$ & $\mathbf{F}$ & $\mathbf{F}$ & $\mathbf{F}$ & $F$ & $\mathbf{F}$ & B \\
\hline B & $\mathbf{F}$ & $\mathbf{F}$ & $\mathbf{F}$ & $\mathbf{F}$ & $\mathbf{F}$ & $\mathbf{F}$ & $\mathbf{F}$ & $\mathbf{F}$ & $\mathbf{F}$ & $\mathbf{F}$ & $\mathbf{F}$ & $\mathbf{F}$ & B \\
\hline B & $\mathbf{F}$ & $\mathbf{F}$ & $\mathbf{F}$ & $\mathbf{F}$ & $\mathbf{F}$ & $\mathbf{F}$ & $\mathbf{F}$ & $\mathbf{F}$ & $\mathbf{F}$ & $\mathbf{F}$ & $\mathbf{F}$ & $\mathbf{F}$ & B \\
\hline B & B & B & $E$ & B & B & B & B & B & B & B & B & B & B \\
\hline
\end{tabular}

Figura 3.1: a) Posição das varáveis em uma célula. b) tipos de células no domínio computacional. 
As equações correspondentes às componentes da velocidade $u$ e $v$ são aplicadas nas faces $\left(i+\frac{1}{2}, j\right)$ e $\left(i, j+\frac{1}{2}\right)$, respectivamente, enquanto que as equações referentes às componentes do tensor tensão e do tensor de Finger são calculadas no centro da célula. Essas equações são aproximadas por diferenças finitas como segue: a derivada temporal é aproximada por um método explícito, enquanto que as derivadas espaciais são aproximadas por diferenças centrais. Os termos convectivos são aproximados pelo método 'upwind' de alta ordem CUBISTA desenvolvido por Alves et al. [2]. Detalhes desse método são apresentados na seção 3.7.3. Logo, a equação (3.34) é aproximada por

$$
\begin{aligned}
\tilde{u}_{i+(1 / 2), j}= & u_{i+(1 / 2), j}+\delta t\left[-\operatorname{conv}\left(u^{2}\right)-\operatorname{conv}(v u)-\frac{1}{\delta x}\left(\tilde{p}_{i+1, j}-\tilde{p}_{i, j}\right)\right. \\
& +\frac{1}{R e}\left(\frac{u_{i-(1 / 2), j}-2 u_{i+(1 / 2), j}+u_{i+(3 / 2), j}}{(\delta x)^{2}}+\frac{u_{i+(1 / 2), j-1}-2 u_{i+(1 / 2), j}+u_{i+(1 / 2), j+1}}{(\delta y)^{2}}\right) \\
& \left.+\frac{S_{i+1, j}^{x x}-S_{i, j}^{x x}}{\delta x}+\frac{S_{i+(1 / 2), j+(1 / 2)}^{x y}-S_{i+(1 / 2), j-(1 / 2)}^{x y}}{\delta y}+\frac{1}{F r^{2}} g_{x}\right]
\end{aligned}
$$

com

$$
\begin{aligned}
S_{i+(1 / 2), j+(1 / 2)}^{x y} & =\frac{1}{4}\left(S_{i, j}^{x y}+S_{i+1, j}^{x y}+S_{i, j+1}^{x y}+S_{i+1, j+1}^{x y}\right) \\
S_{i+(1 / 2), j-(1 / 2)}^{x y} & =\frac{1}{4}\left(S_{i, j}^{x y}+S_{i+1, j}^{x y}+S_{i, j-1}^{x y}+S_{i+1, j-1}^{x y}\right)
\end{aligned}
$$

e a equação (3.35) é aproximada por

$$
\begin{aligned}
\tilde{v}_{i, j+(1 / 2)}= & v_{i, j+(1 / 2)}+\delta t\left[-\operatorname{conv}(u v)-\operatorname{conv}\left(v^{2}\right)-\frac{1}{\delta y}\left(\tilde{p}_{i, j+1}-\tilde{p}_{i, j}\right)\right. \\
& +\frac{1}{R e}\left(\frac{v_{i+1, j+(1 / 2)}-2 v_{i, j+(1 / 2)}+v_{i-1, j+(1 / 2)}}{(\delta x)^{2}}+\frac{v_{i, j-(1 / 2)}-2 v_{i, j+(1 / 2)}+v_{i, j+(3 / 2)}}{(\delta y)^{2}}\right) \\
& \left.+\frac{S_{i+(1 / 2), j+(1 / 2)}^{x y}-S_{i-(1 / 2), j+(1 / 2)}^{x y}}{\delta x}+\frac{S_{i, j+1}^{y y}-S_{i, j}^{y y}}{\delta y}+\frac{1}{F r^{2}} g_{y}\right]
\end{aligned}
$$

com

$$
\begin{gathered}
S_{i+(1 / 2), j+(1 / 2)}^{x y}=\frac{1}{4}\left(S_{i, j}^{x y}+S_{i+1, j}^{x y}+S_{i, j+1}^{x y}+S_{i+1, j+1}^{x y}\right) \\
S_{i-(1 / 2), j+(1 / 2)}^{x y}=\frac{1}{4}\left(S_{i, j}^{x y}+S_{i, j+1}^{x y}+S_{i-1, j}^{x y}+S_{i-1, j+1}^{x y}\right) .
\end{gathered}
$$


Os termos convectivos $\operatorname{conv}\left(u^{2}\right)=\frac{\partial\left(u^{2}\right)}{\partial x}, \operatorname{conv}(v u)=\frac{\partial(v u)}{\partial y}, \operatorname{conv}(u v)=\frac{\partial(u v)}{\partial x} \mathrm{e}$ $\operatorname{conv}\left(v^{2}\right)=\frac{\partial\left(v^{2}\right)}{\partial y}$ são aproximadas pelo método de alta ordem CUBISTA (Alves et al. [2]).

A equação (3.36) é aproximada por diferenças centrais, ou seja,

$$
\frac{\psi_{i+1, j}-2 \psi_{i, j}+\psi_{i-1, j}}{(\delta x)^{2}}+\frac{\psi_{i, j+1}-2 \psi_{i, j}+\psi_{i, j-1}}{(\delta y)^{2}}=\frac{\widetilde{u}_{i+(1 / 2), j}-\widetilde{u}_{i-(1 / 2), j}}{\delta x}+\frac{\widetilde{v}_{i, j+(1 / 2)}-\widetilde{v}_{i, j-(1 / 2)}}{\delta y}
$$

A equação (3.46) é aplicada no centro das células cheias dando origem a um sistema linear simétrico e positivo definido e é resolvido pelo método dos gradientes conjugados.

A velocidade final é obtida pela discretização de (3.37) nos respectivos nós. Assim temos

$$
\left\{\begin{array}{lll}
u_{i+(1 / 2), j}^{n+1} & =\tilde{u}_{i+(1 / 2), j}-\left(\frac{\psi_{i+1, j}-\psi_{i, j}}{\delta x}\right) \\
v_{i, j+(1 / 2)}^{n+1} & = & \tilde{v}_{i, j+(1 / 2)}-\left(\frac{\psi_{i, j+1}-\psi_{i, j}}{\delta y}\right)
\end{array}\right.
$$

A pressão é obtida aplicando (3.38) no centro da célula:

$$
p_{i, j}=\tilde{p}_{i, j}-\frac{\psi_{i, j}}{\delta t}
$$

\subsubsection{Aproximação do tensor de Finger}

O tensor de Finger é calculado pelas equações (2.21)-(2.23) que são discretizadas no centro das células. As derivadas $\frac{\partial u}{\partial x}, \frac{\partial v}{\partial y}, \frac{\partial u}{\partial y}$ and $\frac{\partial v}{\partial x}$ são calculadas usando diferenças centrais e o avanço no tempo é feito com o método de Euler explícito. Os termos convectivos em (2.21) -(2.23) são calculados pelo método upwind CUBISTA (ver Alves et al. [2]). Deste modo as componentes do tensor de Finger são calculadas pelas equações

$$
\begin{aligned}
B_{t_{k}^{\prime}}^{x x}\left(t_{n+1}\right)= & B_{t_{k}^{\prime}}^{x x}\left(t_{n}\right)+\delta t\left\{-\operatorname{conv}\left(u B_{t_{k}^{\prime}}^{x x}\left(t_{n}\right)\right)-\operatorname{conv}\left(v B_{t_{k}^{\prime}}^{x x}\left(t_{n}\right)\right)\right. \\
& \left.+2\left[\left.\left.\frac{\partial u}{\partial x}\right|_{i, j} B_{t_{k}^{\prime}}^{x x}\left(t_{n}\right)\right|_{i, j}+\left.\left.\frac{\partial u}{\partial y}\right|_{i, j} B_{t_{k}^{\prime}}^{x y}\left(t_{n}\right)\right|_{i, j}\right]\right\} \\
B_{t_{k}^{\prime}}^{x y}\left(t_{n+1}\right)= & B_{t_{k}^{\prime}}^{x y}\left(t_{n}\right)+\delta t\left\{-\operatorname{conv}\left(u B_{t_{k}^{\prime}}^{x y}\left(t_{n}\right)\right)-\operatorname{conv}\left(v B_{t_{k}^{\prime}}^{x y}\left(t_{n}\right)\right)\right. \\
& \left.+\left.\left.\frac{\partial v}{\partial x}\right|_{i, j} B_{t_{k}^{\prime}}^{x x}\left(t_{n}\right)\right|_{i, j}+\left.\left.\frac{\partial u}{\partial y}\right|_{i, j} B_{t_{k}^{\prime}}^{y y}\left(t_{n}\right)\right|_{i, j}\right\} \\
B_{t_{k}^{\prime}}^{y y}\left(t_{n+1}\right)= & B_{t_{k}^{\prime}}^{y y}\left(t_{n}\right)+\delta t\left\{-\operatorname{conv}\left(u B_{t_{n}-s_{k}}^{y y}\right)-\operatorname{conv}\left(v B_{t_{n}-s_{k}}^{y y}\right)\right. \\
& \left.+2\left[\left.\left.\frac{\partial v}{\partial x}\right|_{i, j} B_{t_{k}^{\prime}}^{x y}\left(t_{n}\right)\right|_{i, j}+\left.\left.\frac{\partial v}{\partial y}\right|_{i, j} B_{t_{k}^{\prime}}^{y y}\left(t_{n}\right)\right|_{i, j}\right]\right\}
\end{aligned}
$$


onde

$$
\begin{array}{cl}
\operatorname{conv}\left(u B_{t_{k}^{\prime}}^{x x}\left(t_{n}\right)\right)=\frac{\partial}{\partial x}\left(u B_{t_{k}^{\prime}}^{x x}\left(t_{n}\right)\right), \operatorname{conv}\left(v B_{t_{k}^{\prime}}^{x x}\left(t_{n}\right)\right)=\frac{\partial}{\partial y}\left(v B_{t_{k}^{\prime}}^{x x}\left(t_{n}\right)\right), \\
\operatorname{conv}\left(u B_{t_{k}^{\prime}}^{x y}\left(t_{n}\right)\right)=\frac{\partial}{\partial x}\left(u B_{t_{k}^{\prime}}^{x y}\left(t_{n}\right)\right), \operatorname{conv}\left(v B_{t_{k}^{\prime}}^{x y}\left(t_{n}\right)\right)=\frac{\partial}{\partial y}\left(v B_{t_{k}^{\prime}}^{x y}\left(t_{n}\right)\right), \\
\operatorname{conv}\left(u B_{t_{k}^{\prime}}^{y y}\left(t_{n}\right)\right)=\frac{\partial}{\partial x}\left(u B_{t_{k}^{\prime}}^{y y}\left(t_{n}\right)\right), \operatorname{conv}\left(v B_{t_{k}^{\prime}}^{y y}\left(t_{n}\right)\right)=\frac{\partial}{\partial y}\left(v B_{t_{k}^{\prime}}^{y y}\left(t_{n}\right)\right), \\
\left.\frac{\partial u}{\partial x}\right|_{i, j}=\frac{\left(u_{i+1 / 2, j}-u_{i-1 / 2, j}\right)}{\delta x}, & \left.\frac{\partial v}{\partial y}\right|_{i, j}=\frac{\left(v_{i, j+1 / 2}-v_{i, j-1 / 2}\right)}{\delta y}, \\
\left.\frac{\partial u}{\partial y}\right|_{i, j}=\frac{\left(u_{i, j+1 / 2}-u_{i, j-1 / 2}\right)}{\delta y}, & \left.\frac{\partial v}{\partial x}\right|_{i, j}=\frac{\left(v_{i+1 / 2, j}-v_{i-1 / 2, j}\right)}{\delta x} .
\end{array}
$$

Termos não definidos no centro da célula são obtidos por média aritmética. Por exemplo,

$$
\begin{aligned}
& u_{i, j+\frac{1}{2}}=0.25\left(u_{i+\frac{1}{2}, j}+u_{i+\frac{1}{2}, j+1}+u_{i-\frac{1}{2}, j}+u_{i-\frac{1}{2}, j+1}\right) \\
& v_{i+\frac{1}{2}, j}=0.25\left(v_{i, j+\frac{1}{2}}+u_{i+1, j+\frac{1}{2}}+v_{i, j-\frac{1}{2}}+u_{i+1, j-\frac{1}{2}}\right) .
\end{aligned}
$$

As equações (3.49)-(3.51) são resolvidas em cada célula cheia e para cada tempo $t_{k}^{\prime}$, $k=0,1,2, \cdots, N$.

\subsubsection{Aproximação do tensor de Finger nos contornos da malha}

O cálculo dos termos convectivos das equações (3.49) -(3.51) exige que o valor das componentes do tensor de Finger sejam conhecidos no centro das células dos contornos da malha. Esses valores são obtidos como segue:

\section{Contornos rígidos}

Os seguintes casos serão considerados:

i) Contornos rígidos verticais: Esses contornos são identificados por células de contorno tendo a face direita ou a face esquerda em contato com uma face de célula interior (célula F ou S). Nesse caso, as componentes do tensor de Finger são calculadas pelas equações (ver seção 3.4.1)

$$
\frac{\partial}{\partial t} B_{t^{\prime}}^{x x}=0 \quad \frac{\partial}{\partial t} B_{t^{\prime}}^{x y}=\frac{\partial v}{\partial x} B_{t^{\prime}}^{x x} \quad \frac{\partial}{\partial t} B_{t^{\prime}}^{y y}=2 \frac{\partial v}{\partial x} B_{t^{\prime}}^{x y}
$$


Considerando a figura 3.2, essas equações são aproximadas por:

$$
\begin{aligned}
\left.B_{t^{\prime}}^{x x}\left(t_{n+1}\right)\right|_{\left(i+\frac{1}{2}, j\right)} & =1 \\
\left.B_{t^{\prime}}^{x y}\left(t_{n+1}\right)\right|_{\left(i+\frac{1}{2}, j\right)} & =\left.B_{t^{\prime}}^{x y}\left(t_{n}\right)\right|_{\left(i+\frac{1}{2}, j\right)}+\left.\delta t \frac{\partial v}{\partial x}\left(t_{n+1}\right)\right|_{\left(i+\frac{1}{2}, j\right)} \\
\left.B_{t^{\prime}}^{y y}\left(t_{n+1}\right)\right|_{\left(i+\frac{1}{2}, j\right)} & =\left.B_{t^{\prime}}^{y y}\left(t_{n}\right)\right|_{\left(i+\frac{1}{2}, j\right)}+\left.\left.2 \delta t \frac{\partial v}{\partial x}\left(t_{n+1}\right)\right|_{\left(i+\frac{1}{2}, j\right)} B_{t^{\prime}}^{x y}\left(t_{n}\right)\right|_{\left(i+\frac{1}{2}, j\right)}
\end{aligned}
$$

onde $\frac{\partial v}{\partial x}$ é aproximada pela fórmula de 2 a. ordem

$$
\left.\frac{\partial v}{\partial x}\left(t_{n+1}\right)\right|_{\left(i+\frac{1}{2}, j\right)}=\left(3 v_{1}-v_{2} / 3\right) / \delta x
$$

em que

$$
v_{1}=\frac{v_{i+1, j+\frac{1}{2}}+v_{i+1, j-\frac{1}{2}}}{2}, \quad v_{2}=\frac{v_{i+2, j+\frac{1}{2}}+v_{i+2, j-\frac{1}{2}}}{2}
$$

Os valores das componentes do tensor de Finger no centro da célula $B_{i j}$ são então obtidos fazendo interpolação linear utilizando os valores nos pontos $(i+1 / 2, j)$ e $(i+1, j)$, fornecendo

$$
\begin{aligned}
& \left.B_{t^{\prime}}^{x x}\left(t_{n+1}\right)\right|_{(i, j)}=\left.2 B_{t^{\prime}}^{x x}\left(t_{n+1}\right)\right|_{\left(i+\frac{1}{2}, j\right)}-\left.B_{t^{\prime}}^{x x}\left(t_{n+1}\right)\right|_{(i+1, j)} \\
& \left.B_{t^{\prime}}^{x y}\left(t_{n+1}\right)\right|_{(i, j)}=\left.2 B_{t^{\prime}}^{x y}\left(t_{n+1}\right)\right|_{\left(i+\frac{1}{2}, j\right)}-\left.B_{t^{\prime}}^{x y}\left(t_{n+1}\right)\right|_{(i+1, j)} \\
& \left.B_{t^{\prime}}^{y y}\left(t_{n+1}\right)\right|_{(i, j)}=\left.2 B_{t^{\prime}}^{y y}\left(t_{n+1}\right)\right|_{\left(i+\frac{1}{2}, j\right)}-\left.B_{t^{\prime}}^{y y}\left(t_{n+1}\right)\right|_{(i+1, j)}
\end{aligned}
$$

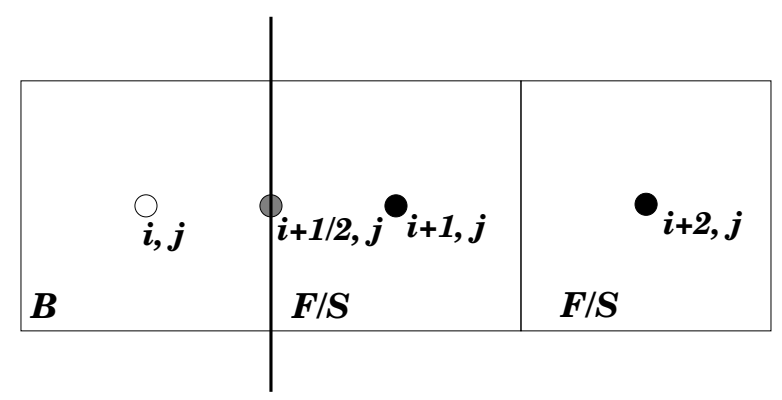

Figura 3.2: Cálculo das componentes do tensor de Finger no centro da célula de contorno $B_{i j}$ : contornos verticais.

ii) Contornos rígidos horizontais: Esses contornos são identificados por células de contorno tendo a face superior ou a face inferior em contato com uma face de célula interior (célula F ou S). Nesse caso, as componentes do tensor de Finger são calculadas pelas equações (ver seção 3.4.1)

$$
\frac{\partial}{\partial t} B_{t^{\prime}}^{x x}=2 \frac{\partial u}{\partial y} B_{t^{\prime}}^{x y}, \quad \frac{\partial}{\partial t} B_{t^{\prime}}^{x y}=\frac{\partial u}{\partial y} B_{t^{\prime}}^{y y}, \quad \frac{\partial}{\partial t} B_{t^{\prime}}^{y y}=0 .
$$


Considerando a figura 3.3 essas equações são aproximadas por:

$$
\begin{aligned}
\left.B_{t^{\prime}}^{y y}\left(t_{n+1}\right)\right|_{\left(i, j+\frac{1}{2}\right)} & =1 \\
\left.B_{t^{\prime}}^{x y}\left(t_{n+1}\right)\right|_{\left(i, j+\frac{1}{2}\right)} & =\left.B_{t^{\prime}}^{x y}\left(t_{n}\right)\right|_{\left(i, j+\frac{1}{2}\right)}+\left.\delta t \frac{\partial u}{\partial y}\left(t_{n+1}\right)\right|_{\left(i, j+\frac{1}{2}\right)} \\
\left.B_{t^{\prime}}^{x x}\left(t_{n+1}\right)\right|_{\left(i, j+\frac{1}{2}\right)} & =\left.B_{t^{\prime}}^{x x}\left(t_{n}\right)\right|_{\left(i, j+\frac{1}{2}\right)}+\left.\left.2 \delta t \frac{\partial u}{\partial y}\left(t_{n+1}\right)\right|_{\left(i, j+\frac{1}{2}\right)} B_{t^{\prime}}^{x y}\left(t_{n}\right)\right|_{\left(i, j+\frac{1}{2}\right)}
\end{aligned}
$$

onde $\frac{\partial u}{\partial y}$ é aproximada pela fórmula de $2 \mathrm{a}$. ordem

$$
\left.\frac{\partial u}{\partial y}\left(t_{n+1}\right)\right|_{\left(i, j+\frac{1}{2}\right)}=\left(3 u_{1}-u_{2} / 3\right) / \delta y
$$

em que

$$
u_{1}=\frac{u_{i+\frac{1}{2}, j+1}+u_{i-\frac{1}{2}, j+1}}{2}, \quad u_{2}=\frac{u_{i+\frac{1}{2}, j+2}+u_{i-\frac{1}{2}, j+2}}{2}
$$

Os valores das componentes do tensor de Finger no centro da célula $B_{i j}$ são então obtidos fazendo interpolação linear utilizando os valores nos pontos $(i+1 / 2, j)$ e $(i+1, j)$, fornecendo

$$
\begin{aligned}
& \left.B_{t^{\prime}}^{x x}\left(t_{n+1}\right)\right|_{(i, j)}=\left.2 B_{t^{\prime}}^{x x}\left(t_{n+1}\right)\right|_{\left(i, j+\frac{1}{2}\right)}-\left.B_{t^{\prime}}^{x x}\left(t_{n+1}\right)\right|_{(i, j+1)} \\
& \left.B_{t^{\prime}}^{x y}\left(t_{n+1}\right)\right|_{(i, j)}=\left.2 B_{t^{\prime}}^{x y}\left(t_{n+1}\right)\right|_{\left(i, j+\frac{1}{2}\right)}-\left.B_{t^{\prime}}^{x y}\left(t_{n+1}\right)\right|_{(i, j+1)} \\
& \left.B_{t^{\prime}}^{y y}\left(t_{n+1}\right)\right|_{(i, j)}=\left.2 B_{t^{\prime}}^{y y}\left(t_{n+1}\right)\right|_{\left(i, j+\frac{1}{2}\right)}-\left.B_{t^{\prime}}^{y y}\left(t_{n+1}\right)\right|_{(i, j+1)}
\end{aligned}
$$

Células de contorno com a face inferior em contato com células interiores são tratadas de maneira semelhante.

iii) Contornos rígidos com "quinas": Esses contornos são definidos por células de contorno B que possuem duas faces adjacentes em contanto com células interiores. Para problemas bidimensionais, a figura 3.4 mostra as situações possíveis de contornos tipo "quinas". Neste caso, dois valores para as componentes do tensor de Finger no centro da célula são encontrados: um usando a face horizontal em contato com a face da célula de interior e outro utilizando a face vertical em contato com a face da célula interior (ver itens i) e ii) acima). O valor final será dado pela média dos valores encontrados. Por exemplo, considerando a configuração da figura 3.4 , os valores das componentes do tensor de Finger no centro da célula de contorno são 


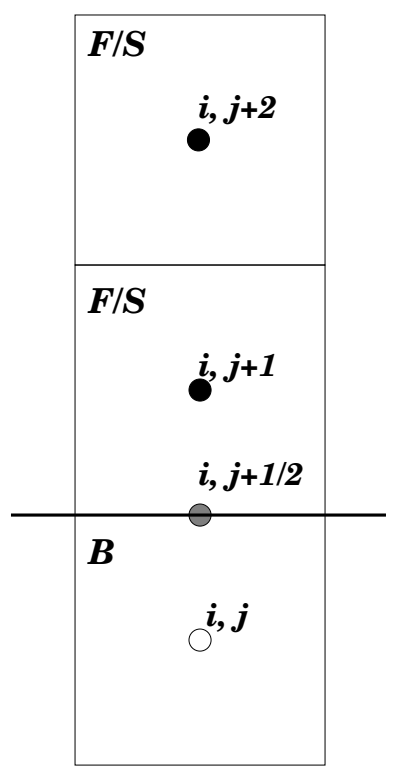

Figura 3.3: Cálculo das componentes do tensor de Finger no centro da célula de contorno $B_{i j}$ : contornos horizontais.

calculados por

$$
\begin{aligned}
\left.B_{t^{\prime}}^{x x}\left(t_{n+1}\right)\right|_{(i, j)}= & \left.B_{t^{\prime}}^{x x}\left(t_{n+1}\right)\right|_{\left(i, j+\frac{1}{2}\right)}+\left.B_{t^{\prime}}^{x x}\left(t_{n+1}\right)\right|_{\left(i+\frac{1}{2}, j\right)} \\
& -\frac{1}{2}\left[\left.B_{t^{\prime}}^{x x}\left(t_{n+1}\right)\right|_{(i, j+1)}+\left.B_{t^{\prime}}^{x x}\left(t_{n+1}\right)\right|_{(i+1, j)}\right] \\
\left.B_{t^{\prime}}^{x y}\left(t_{n+1}\right)\right|_{(i, j)}= & \left.B_{t^{\prime}}^{x y}\left(t_{n+1}\right)\right|_{\left(i, j+\frac{1}{2}\right)}+\left.B_{t^{\prime}}^{x y}\left(t_{n+1}\right)\right|_{\left(i+\frac{1}{2}, j\right)} \\
& -\frac{1}{2}\left[\left.B_{t^{\prime}}^{x y}\left(t_{n+1}\right)\right|_{(i, j+1)}+\left.B_{t^{\prime}}^{x y}\left(t_{n+1}\right)\right|_{(i+1, j)}\right] \\
\left.B_{t^{\prime}}^{y y}\left(t_{n+1}\right)\right|_{(i, j)}= & \left.B_{t^{\prime}}^{y y}\left(t_{n+1}\right)\right|_{\left(i, j+\frac{1}{2}\right)}+\left.B_{t^{\prime}}^{y y}\left(t_{n+1}\right)\right|_{\left(i+\frac{1}{2}, j\right)} \\
& -\frac{1}{2}\left[\left.B_{t^{\prime}}^{y y}\left(t_{n+1}\right)\right|_{(i, j+1)}+\left.B_{t^{\prime}}^{y y}\left(t_{n+1}\right)\right|_{(i+1, j)}\right]
\end{aligned}
$$

\section{Injetores}

Nas células de injetores, nas quais a velocidade é conhecida, o tensor de Finger é calculado exatamente, considerando que o escoamento nessas células é cisalhante, com taxa de cisalhamento igual a $\frac{\partial u}{\partial y}$ para injetores verticais e $\frac{\partial u}{\partial x}$ para injetores horizontais (veja seção $3.4 .2)$.

\section{Ejetores}

i) face ejetora é paralela ao eixo $x$ : 
a)

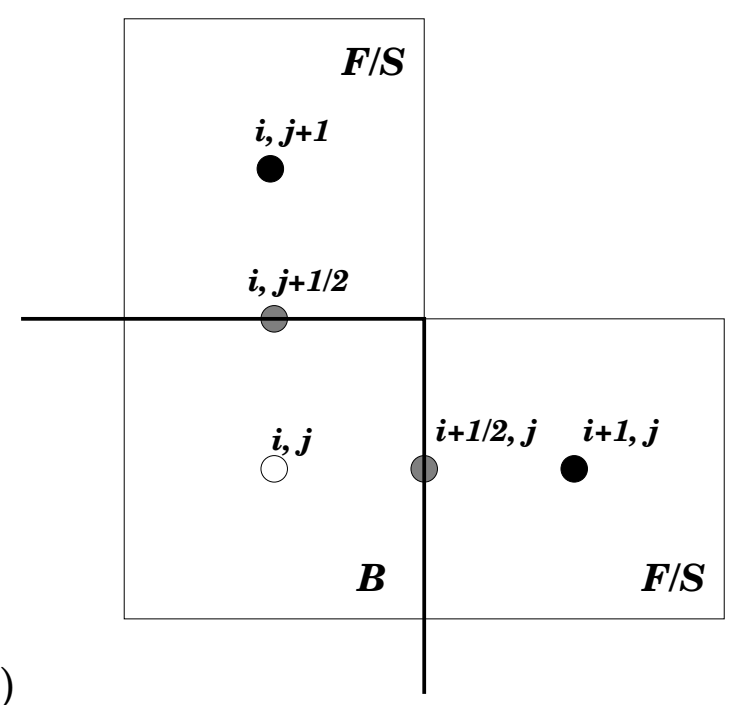

c)

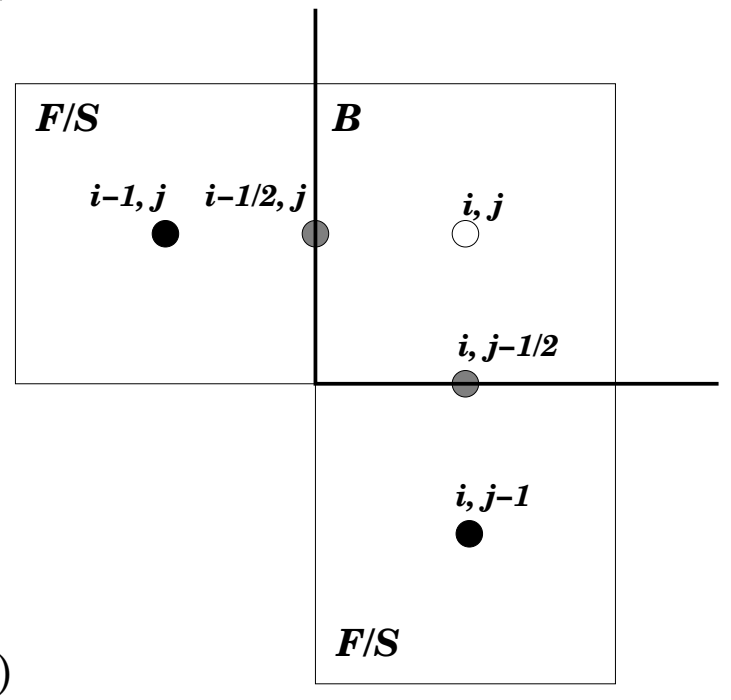

b)

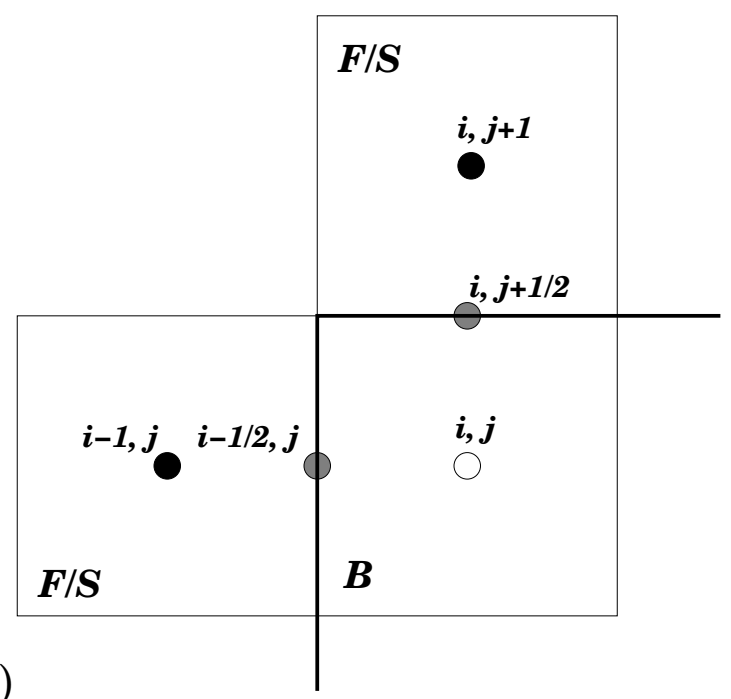

d)

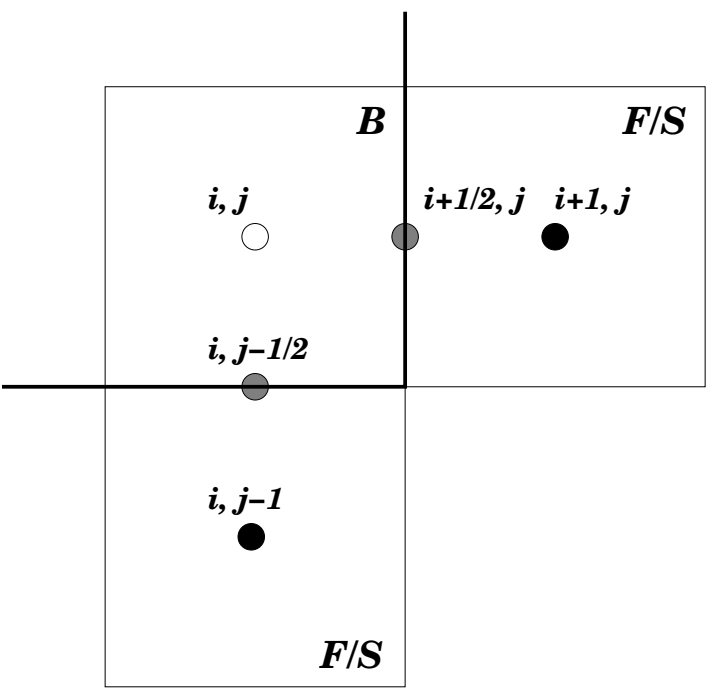

Figura 3.4: Cálculo das componentes do tensor de Finger centro da célula de contorno : contornos de "quina"

Se o ejetor for a face inferior de uma célula (figura $3.5 \mathrm{a}$ ) temos

$$
\begin{aligned}
& \left.B_{t^{\prime}}^{x x}\right|_{(i, j)}=\left.B_{t^{\prime}}^{x x}\right|_{(i, j-1)} \\
& \left.B_{t^{\prime}}^{x y}\right|_{(i, j)}=\left.B_{t^{\prime}}^{x y}\right|_{(i, j-1)} \\
& \left.B_{t^{\prime}}^{y y}\right|_{(i, j)}=\left.B_{t^{\prime}}^{y y}\right|_{(i, j-1)}
\end{aligned}
$$

caso contrário, se o ejetor for a face superior de uma célula (figura $3.5 \mathrm{~b}$ ) temos

$$
\begin{aligned}
& \left.B_{t^{\prime}}^{x x}\right|_{(i, j)}=\left.B_{t^{\prime}}^{x x}\right|_{(i, j+1)} \\
& \left.B_{t^{\prime}}^{x y}\right|_{(i, j)}=\left.B_{t^{\prime}}^{x y}\right|_{(i, j+1)} \\
& \left.B_{t^{\prime}}^{y y}\right|_{(i, j)}=\left.B_{t^{\prime}}^{y y}\right|_{(i, j+1)}
\end{aligned}
$$

ii) face ejetora é paralela ao eixo $y$ : 
a)

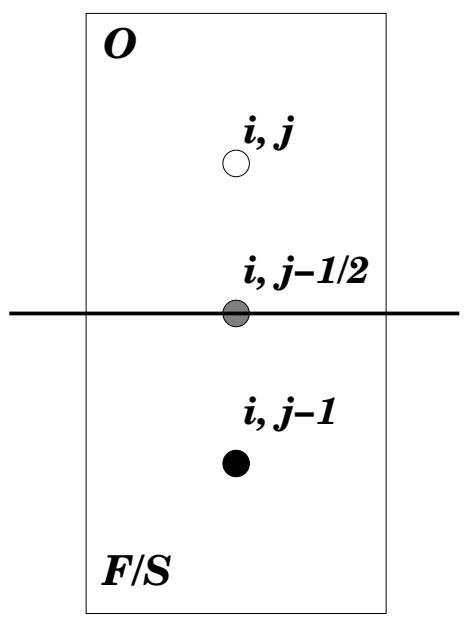

b)

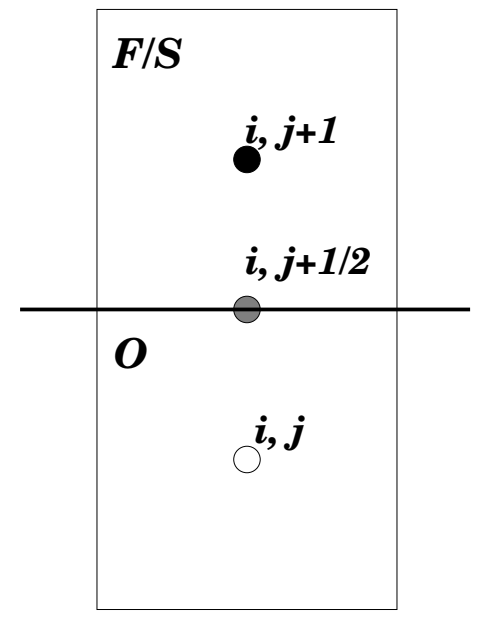

Figura 3.5: Cálculo das componentes do tensor de Finger no centro da células ejetoras. Feces ejetoras horizontais

Para ejetor sendo a face $(i+1 / 2, j)$ (figura 3.6 a) temos

$$
\begin{aligned}
& \left.B_{t^{\prime}}^{x x}\right|_{(i, j)}=\left.B_{t^{\prime}}^{x x}\right|_{(i+1, j)} \\
& \left.B_{t^{\prime}}^{x y}\right|_{(i, j)}=\left.B_{t^{\prime}}^{x y}\right|_{(i+1, j)} \\
& \left.B_{t^{\prime}}^{y y}\right|_{(i, j)}=\left.B_{t^{\prime}}^{y y}\right|_{(i+1, j)}
\end{aligned}
$$

e se o ejetor for a face $(i-1 / 2, j)$ (figura $3.5 \mathrm{~b}$ ) temos

$$
\begin{aligned}
& \left.B_{t^{\prime}}^{x x}\right|_{(i, j)}=\left.B_{t^{\prime}}^{x x}\right|_{(i-1, j)} \\
& \left.B_{t^{\prime}}^{x y}\right|_{(i, j)}=\left.B_{t^{\prime}}^{x y}\right|_{(i-1, j)} \\
& \left.B_{t^{\prime}}^{y y}\right|_{(i, j)}=\left.B_{t^{\prime}}^{y y}\right|_{(i-1, j)}
\end{aligned}
$$

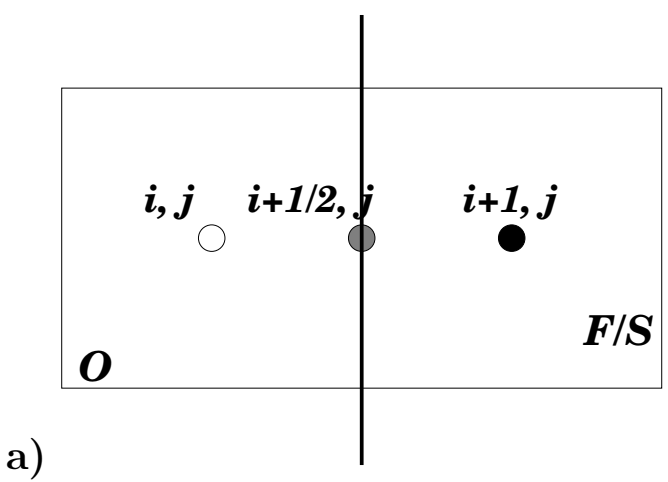

b)

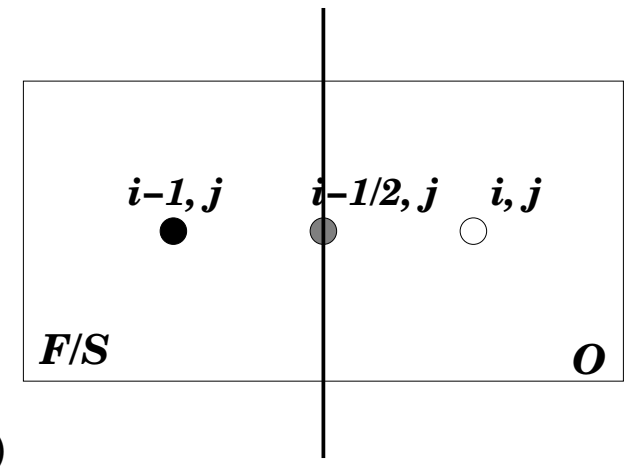

Figura 3.6: Cálculo das componentes do tensor de Finger no centro da células ejetoras. Feces ejetoras verticais 


\subsubsection{Aproximação dos termos convectivos}

Nesta tese os termos convectivos nas equações de quantidade de movimento e também no cálculo do tensor de Finger são tratados com o método de alta resolução CUBISTA (Convergent and Universally Bounded Interpolation Scheme for the Treatment of Advection) introduzido por Alves et al. [2] e é de terceira ordem em malhas uniformes. Esse método é resumido a seguir:

Considere a figura abaixo

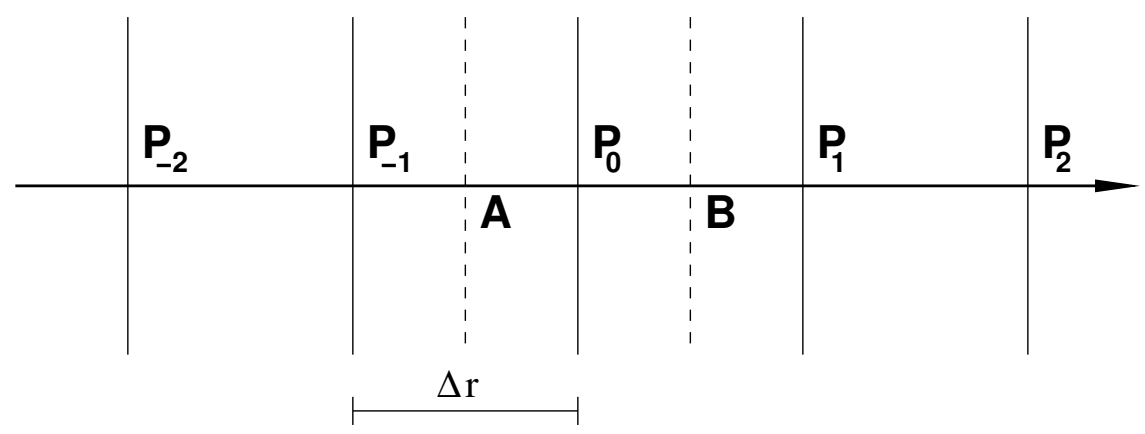

Figura 3.7: Estêncil utilizado para calcular $\phi$ nos pontos A e B.

Seja $\frac{\partial(V \phi)}{\partial r}$ a derivada a ser calculada no ponto $P_{0}$, onde $V(u$ ou $v)$ é a velocidade convectiva, $r$ é a direção ( $x$ ou $y$ no nosso caso) e $\phi$ é a variável genérica a ser convectada (por exemplo, se considerarmos a equação (3.49), então $\phi=B^{x x}$ ). Esta derivada pode ser aproximada por

$$
\left.\frac{\partial(V \phi)}{\partial r}\right|_{P_{0}}=\frac{(V \phi)_{B}-(V \phi)_{A}}{\Delta r}
$$

Considere agora a notação $P_{\mathrm{U}}$ (upstream), $P_{\mathrm{D}}$ (downstream) e $P_{\mathrm{R}}$ (remote-upstream) com relação a um ponto P (A ou B). Seguindo a direção do sinal da velocidade $V$ nesse ponto, $P_{\mathrm{D}}$ será o ponto imediatamente "a frente" de P, $P_{\mathrm{U}}$ será o ponto imediatamente "atrás" de $\mathrm{P}$ e $P_{\mathrm{R}}$ será o ponto imediatamente "atrás" de $P_{\mathrm{U}}$. Por exemplo, se a velocidade $V$ no ponto A for positiva então $P_{\mathrm{D}}, P_{\mathrm{U}}$ e $P_{\mathrm{R}}$ são, respectivamente, os pontos $P_{0}, P_{-1}$ e $P_{-2}$. Caso contrário, se $V<0$ então $P_{\mathrm{D}}=P_{-1}, P_{\mathrm{U}}=P_{0}$ e $P_{\mathrm{R}}=P_{1}$.

Considerando esta convenção, pode-se calcular a variável

$$
\hat{\phi}_{U}=\frac{\phi_{U}-\phi_{R}}{\phi_{D}-\phi_{R}}
$$


O método CUBISTA calcula $\phi_{P}$ pela fórmula

$$
\phi_{P}= \begin{cases}\phi_{U} & \text { se } \hat{\phi}_{U}<0 \\ \frac{7}{4} \phi_{U}-\frac{3}{4} \phi_{R} & \text { se } 0 \leq \hat{\phi}_{U}<\frac{3}{8} \\ \frac{3}{8} \phi_{D}+\frac{6}{8} \phi_{U}-\frac{1}{8} \phi_{R} & \text { se } \frac{3}{8} \leq \hat{\phi}_{U}<\frac{3}{4} \\ \frac{3}{4} \phi_{D}+\frac{1}{4} \phi_{U} & \text { se } \frac{3}{4} \leq \hat{\phi}_{U}<1 \\ \phi_{U} & \text { se } \hat{\phi}_{U} \geq 1\end{cases}
$$

\subsubsection{Aproximação das condições de tensão na superfície livre}

As condições de tensão na superfície livre são dadas pelas expressões (3.31) e (3.33) e utilizando coordenadas cartesianas podem ser escritas como:

$-\widetilde{p}+\frac{2}{R e}\left[\frac{\partial u}{\partial x} n_{x}^{2}+\left(\frac{\partial u}{\partial y}+\frac{\partial v}{\partial x}\right) n_{x} n_{y}+\frac{\partial v}{\partial y} n_{y}^{2}\right]+S^{x x} n_{x}^{2}+2 S^{x y} n_{x} n_{y}+S^{y y} n_{y}^{2}=0$

$\frac{2}{R e}\left[\left(\frac{\partial u}{\partial x}-\frac{\partial v}{\partial y}\right) n_{x} n_{y}+\frac{1}{2}\left(\frac{\partial u}{\partial y}+\frac{\partial v}{\partial x}\right)\left(n_{y}^{2}-n_{x}^{2}\right)\right]+\left(S^{x x}-S^{y y}\right) n_{x} n_{y}+S^{x y}\left(n_{y}^{2}-n_{x}^{2}\right)=0(3.71)$

Para aplicar essas condições seguimos as idéias de Tomé et al. [60]. Assumimos que a malha seja suficientemente fina, de modo que, localmente, a superfície livre possa ser aproximada por uma superfície horizontal, vertical ou com inclinação de $45^{\circ}$ em relação aos eixos coordenados. Para essas superfícies, os vetores normais tomam a forma $\mathbf{n}=( \pm 1,0)$ ou $\mathbf{n}=(0, \pm 1)$ ou $\mathbf{n}=\left( \pm \frac{\sqrt{2}}{2}, \pm \frac{\sqrt{2}}{2}\right)$. Essas superfícies são identificadas por células $\mathrm{S}$ tendo somente uma face em contato com uma célula E (ver figura 3.8) ou células S tendo duas faces adjacentes em contato com faces de células E (ver figura 3.9). Por exemplo, se considerarmos a célula $\mathrm{S}$ tendo a face $\left(j+\frac{1}{2}\right)$ em contato com uma célula E na figura 3.8d então tomamos $\mathbf{n}=(0,1)$ e as equações (3.70)-(3.71) se reduzem a

$$
\begin{aligned}
& -\widetilde{p}+\frac{2}{R e} \frac{\partial v}{\partial y}+S^{y y}=0 \\
& \frac{1}{R e}\left(\frac{\partial u}{\partial y}+\frac{\partial v}{\partial x}\right)+S^{x y}=0 .
\end{aligned}
$$

respectivamente.

Por exemplo, considerando a figura $3.8 \mathrm{~d}$, os valores de $v_{i, j+\frac{1}{2}}, u_{i+\frac{1}{2}, j+1}, \widetilde{p}_{i, j}$ e $\left.\mathbf{B}_{t^{\prime}}\left(t_{n}\right)\right|_{i, j}$ são necessários e calculados como segue. 
a)
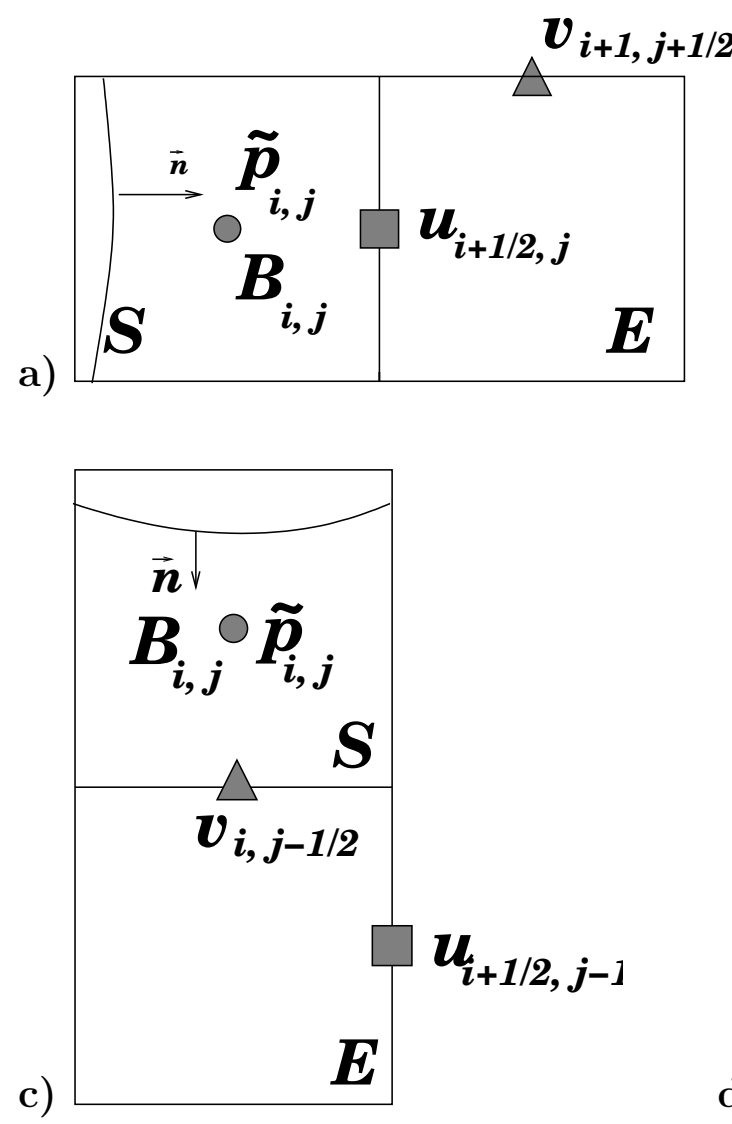

b)
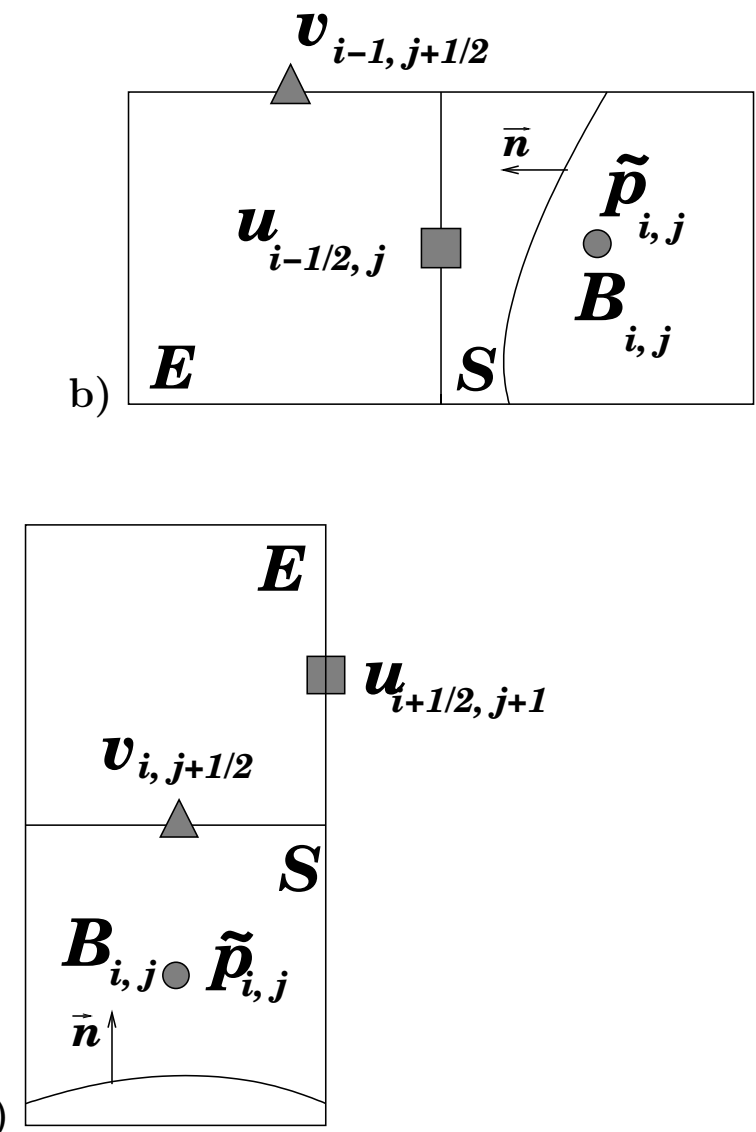

Figura 3.8: Configurações de células (S) com somente uma face em contato com célula $(\mathrm{E})$.

O valor de $v_{i, j+\frac{1}{2}}$ é obtido aplicando-se a equação da continuidade no centro da célula e isolando $v_{i, j+\frac{1}{2}}$ obtém-se

$$
v_{i, j+\frac{1}{2}}=v_{i, j-\frac{1}{2}}-\frac{\delta y}{\delta x}\left(u_{i+\frac{1}{2}, j}-u_{i-\frac{1}{2}, j}\right)
$$

Agora, discretizando a equação (3.73) na posição $\left(i+\frac{1}{2}, j+\frac{1}{2}\right)$, tem-se

$$
\frac{1}{R e}\left(\frac{u_{i+\frac{1}{2}, j+1}-u_{i+\frac{1}{2}, j}}{\delta y}+\frac{\left(v_{i+1, j+\frac{1}{2}}-v_{i, j+\frac{1}{2}}\right)}{\delta x}\right)+S_{i+\frac{1}{2}, j+\frac{1}{2}}^{x y}=0,
$$

e explicitando $u_{i+\frac{1}{2}, j+1}$ obtém-se

$$
u_{i+\frac{1}{2}, j+1}=u_{i+\frac{1}{2}, j}-\frac{\delta y}{\delta x}\left(v_{i+1, j+\frac{1}{2}}-v_{i, j+\frac{1}{2}}\right)-\delta y \operatorname{Re} S_{i+\frac{1}{2}, j+\frac{1}{2}}^{x y} .
$$

A pressão $\widetilde{p}_{i, j}$ é então calculada pela equação (3.72) aplicada no centro da célula, resultando em

$$
\widetilde{p}_{i, j}=\frac{2}{R e} \frac{\left(v_{i, j+\frac{1}{2}}-v_{i, j-\frac{1}{2}}\right)}{\delta y}+S_{i, j}^{y y} .
$$


As componentes do tensor de Finger, $B_{t_{k}^{\prime}}^{x x}, B_{t_{k}^{\prime}}^{x y}$ e $B_{t_{k}^{\prime}}^{y y}$ são calculadas pelas equações (3.49)(3.51) onde a derivada cruzada $\left.\frac{\partial u}{\partial y}\right|_{i, j}$ é aproximada por

$$
\left.\frac{\partial u}{\partial y}\right|_{i, j}=\frac{1}{2} \frac{u_{i+\frac{1}{2}, j}+u_{i-\frac{1}{2}, j}-u_{i+\frac{1}{2}, j-1}-u_{i-\frac{1}{2}, j-1}}{\delta y} .
$$

Outros tipos de células $\mathrm{S}$ tendo somente uma face em contato com uma face de célula E são tratados da mesma forma.

Para células S com duas faces adjacentes em contato com faces de células E, o tratamento é semelhante. Considere por exemplo a célula S mostrada na figura $3.9 \mathrm{a}$

a)

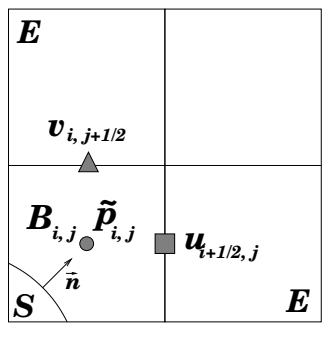

b)

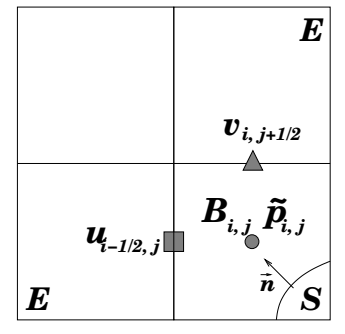

c)

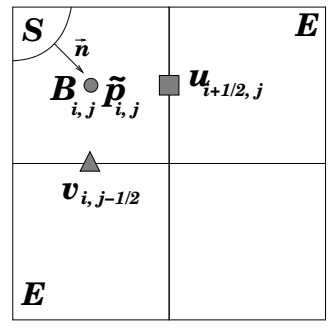

d)

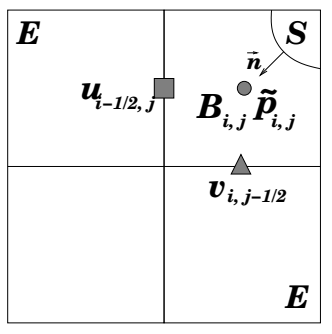

Figura 3.9: Configurações de células (S) com duas faces adjacentes em contato com células (E).

Neste caso, $\mathbf{n}=\left(\frac{\sqrt{2}}{2}, \frac{\sqrt{2}}{2}\right)$ e as condições $(3.70)-(3.71)$ reduzem a

$$
\begin{aligned}
-\widetilde{p}+\frac{1}{R e}\left(\frac{\partial u}{\partial y}+\frac{\partial v}{\partial x}\right)+\frac{1}{2}\left(S^{x x}+2 S^{x y}+S^{y y}\right) & =0, \\
\frac{2}{R e}\left(\frac{\partial u}{\partial x}-\frac{\partial v}{\partial y}\right)+\left(S^{x x}-S^{y y}\right) & =0 .
\end{aligned}
$$

Os valores de $u_{i+\frac{1}{2}, j}$ e $v_{i, j+\frac{1}{2}}$ são obtidos aplicando a equação de conservação de massa e a condição (3.79) no centro da célula. Neste caso obtemos

$$
\begin{array}{r}
\frac{\left(u_{i+\frac{1}{2}, j}-u_{i-\frac{1}{2}, j}\right)}{\delta x}+\frac{\left(v_{i, j+\frac{1}{2}}-v_{i, j-\frac{1}{2}}\right)}{\delta y}=0, \\
\frac{\left(u_{i+\frac{1}{2}, j}-u_{i-\frac{1}{2}, j}\right)}{\delta x}-\frac{\left(v_{i, j+\frac{1}{2}}-v_{i, j-\frac{1}{2}}\right)}{\delta y}=-\frac{R e}{2}\left(S_{i, j}^{x x}-S_{i, j}^{y y}\right) .
\end{array}
$$

As equações (3.80) e (3.81) formam um sistema linear $(2 \times 2)$ nas incógnitas $u_{i+\frac{1}{2}, j}$ e $v_{i, j+\frac{1}{2}}$, cuja solução é dada por

$$
u_{i+\frac{1}{2}, j}=u_{i-\frac{1}{2}, j}-\frac{\delta x}{4} R e\left(S_{i, j}^{x x}-S_{i, j}^{y y}\right), \quad v_{i, j+\frac{1}{2}}=v_{i, j-\frac{1}{2}}-\frac{\delta y}{\delta x}\left(u_{i+\frac{1}{2}, j}-u_{i-\frac{1}{2}, j}\right) .
$$

Após calcular $u_{i+\frac{1}{2}, j}$ e $v_{i, j+\frac{1}{2}}$, a pressão no centro da célula é calculada com (3.78) aplicada no centro da célula, resultando em

$$
\widetilde{p}_{i, j}=\frac{1}{R e}\left[\frac{\left(u_{i, j}-u_{i, j-1}\right)}{\delta y}+\frac{\left(v_{i, j}-v_{i-1, j}\right)}{\delta x}\right]-\frac{1}{2}\left(S_{i, j}^{x x}+2 S_{i, j}^{x y}+S_{i, j}^{y y}\right)
$$


onde os valores de $u_{i, j}$ and $v_{i, j}$ são dados por

$$
u_{i, j}=\frac{u_{i+\frac{1}{2}, j}+u_{i-\frac{1}{2}, j}}{2}, \quad v_{i, j}=\frac{v_{i, j+\frac{1}{2}}+v_{i, j-\frac{1}{2}}}{2} .
$$

Novamente, as componentes do tensor de Finger, $B_{t_{k}^{\prime}}^{x x}, B_{t_{k}^{\prime}}^{x y}$ e $B_{t_{k}^{\prime}}^{y y}$ são calculadas pelas equações (3.49) $-(3.51)$ onde as derivadas cruzadas $\left.\frac{\partial u}{\partial y}\right|_{i, j}$ e $\left.\frac{\partial v}{\partial x}\right|_{i, j}$ são aproximadas por

$$
\begin{aligned}
\left.\frac{\partial u}{\partial y}\right|_{i, j} & =\frac{1}{2} \frac{u_{i+\frac{1}{2}, j}+u_{i-\frac{1}{2}, j}-u_{i+\frac{1}{2}, j-1}-u_{i-\frac{1}{2}, j-1}}{\delta y} \\
\left.\frac{\partial v}{\partial x}\right|_{i, j} & =\frac{1}{2} \frac{v_{i, j+\frac{1}{2}}+v_{i, j-\frac{1}{2}}-v_{i-1, j+\frac{1}{2}}-v_{i-1, j-\frac{1}{2}}}{\delta x}
\end{aligned}
$$

Outras configurações de células $\mathrm{S}$ tendo duas faces adjacentes em contato com faces de células E são tratadas de modo semelhante. Para células de superfície que possuem duas faces opostas em contato com faces de células vazias não há uma aproximação para o vetor normal. Neste caso a pressão $\widetilde{p}_{i, j}$ é tomada igual a zero e a velocidade é ajustada de modo a satisfazer a equação da continuidade nessa célula.

\subsubsection{Cálculo do passo de tempo}

A determinação do passo de tempo leva em consideração o fato de que uma partícula não pode percorrer uma distância maior do que o comprimento de uma célula. Isto exige que em cada ciclo computacional sejam verificadas as condições

$$
\begin{aligned}
\delta t_{u} & \leq \frac{1}{2} \frac{\delta x}{u_{\max }} \\
\delta t_{v} & \leq \frac{1}{2} \frac{\delta y}{v_{\max }}
\end{aligned}
$$

onde $u_{\max }$ e $v_{\max }$ são os módulos máximos das velocidades nas direções $x$ e $y$, respectivamente. Além disso, a seguinte restrição de estabilidade também é verificada [61]

$$
\delta t_{v i s c} \leq \operatorname{Re}\left(\frac{\delta_{x}^{2} \delta_{y}^{2}}{\delta_{x}^{2}+\delta_{y}^{2}}\right)
$$

de modo que o passo de tempo usado na simulação será dado por

$$
\delta t=F A C T \min \left(\delta t_{u}, \delta t_{v}, \delta t_{v i s c}\right), 0 \leq F A C T \leq 1
$$

Detalhes da implementação dessas condições são apresentadas em Tomé e McKee [61]. 


\section{Capítulo 4}

\section{Resultados Numéricos}

As equações de diferenças finitas obtidas na seção 3.7 foram implementadas no sistema FreeFlow-2D com o objetivo de simular escoamentos viscoelásticos bidimensionais governados pelas equações constitutivas integrais de Maxwell e K-BKZ. O código FreeFlow-2D foi utilizado para simular escoamentos confinados: escoamento em um canal bidimensional e o problema da contração 4:1; e escoamentos com superfícies livres: 'jet buckling' . Os resultados são mostrados a seguir.

\subsection{Validação do Método Numérico}

Com o objetivo de validar a técnica numérica apresentada no capítulo anterior, o código FreeFlow-2D foi utilizado para simular o escoamento em um canal bidimensional governado pelos modelos integrais de Maxwell e K-BKZ. Consideramos um canal bidimensional com largura $L$ e comprimento $10 L$, conforme mostrado na figura 4.1 .

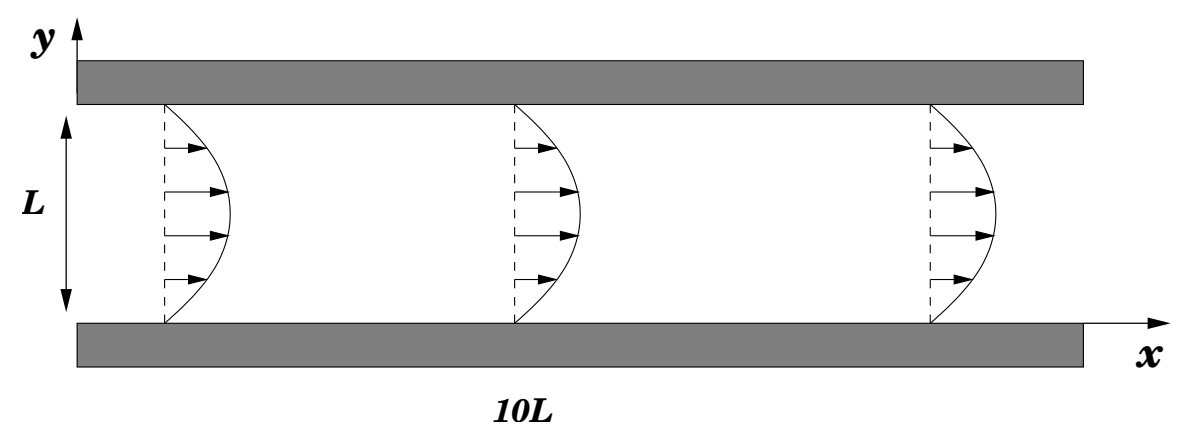

Figura 4.1: Canal bidimensional. 


\subsubsection{Modelo Maxwell}

Para validar a implementação do modelo Maxwell, simulamos o escoamento no canal com os seguintes dados de entrada:

- Velocidade de escala: $U=1 \mathrm{~ms}^{-1}$,

- Escala de comprimento: $L=0,01 \mathrm{~m}$,

- Viscosidade : $\nu=0,01 \mathrm{~m}^{2} \mathrm{~s}^{-1}$

- Definição do modelo de Maxwell: $a=1000 \mathrm{~Pa}$ e $\lambda=0,01 \mathrm{~s}$,

- Precisão na equação de Poisson: $\mathrm{EPS}=10^{-10}$,

- Sem efeito gravitacional, $g=0$.

Logo, temos $R e=U L / \nu=1$ e $W e=\lambda U / L=1$.

Para analisar a convergência do método numérico, esse problema foi simulado utilizando quatro malhas diferentes: Malha M1 com $10 \times 100$ células, Malha M2 com $20 \times 200$ células, Malha M3 com $30 \times 300$ células e Malha M4 com $40 \times 400$ células .

A simulação começou com o canal cheio de fluido com velocidade nula e o tensor de Finger foi tomado como sendo o tensor identidade em todas as células cheias. Nas paredes do canal foi imposta a condição de não-escorregamento para a velocidade e na saída do canal as condições de Neumann.

Na entrada do canal foi imposto um perfil parabólico para a velocidade dado por:

$$
u(y)=-4(y-0,5)^{2}+1
$$

e o tensor de Finger foi calculado utilizando as equações

$$
B_{t^{\prime}}^{x x}(t)=1+\left(\frac{\partial u}{\partial y}\right)^{2}\left(t-t^{\prime}\right)^{2}, \quad B_{t^{\prime}}^{x y}(t)=\left(\frac{\partial u}{\partial y}\right)^{2}\left(t-t^{\prime}\right), \quad B_{t^{\prime}}^{y y}(t)=1 .
$$

Lembramos que, nesse caso, as tensões podem ser calculadas analiticamente e são dadas por (ver seção 2.4):

$$
\begin{aligned}
\tau^{x x}(t) & =a e^{\frac{-t}{W e}}\left(1+\dot{\gamma}^{2} t^{2}\right)+a\left(1-e^{\frac{-t}{W e}}\right)+a \dot{\gamma}^{2}\left[2(W e)^{2}-e^{\frac{-t}{W e}}\left(t^{2}+2 W e t+2(W e)^{2}\right)\right] \\
\tau^{x y}(t) & =a \dot{\gamma} W e\left(1-e^{\frac{-t}{W e}}\right) \\
\tau^{y y}(t) & =\tau^{z z}(t)=a .
\end{aligned}
$$


$\operatorname{com} \dot{\gamma}=\frac{\partial u}{\partial y}$

O código FreeFlow-2D simulou esse problema utilizando as quatro malhas até o tempo $t=100$. A figura 4.2 mostra as linhas de nível para a componente da velocidade $u$ e a componente da tensão $\tau^{x x}$ em $t=100$, onde podemos observar que as linhas de nível estão paralelas, o que indica que o estado estacionário foi alcançado.

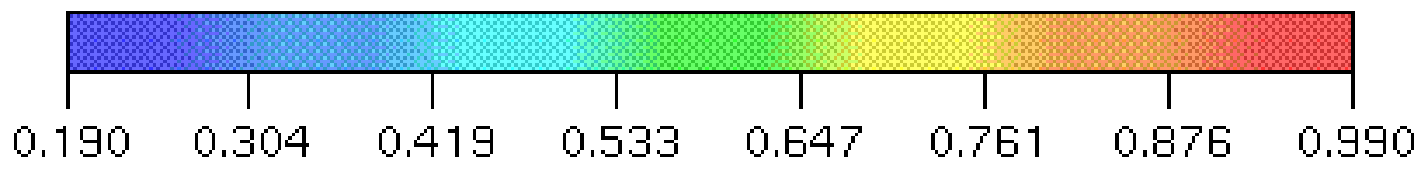

a)

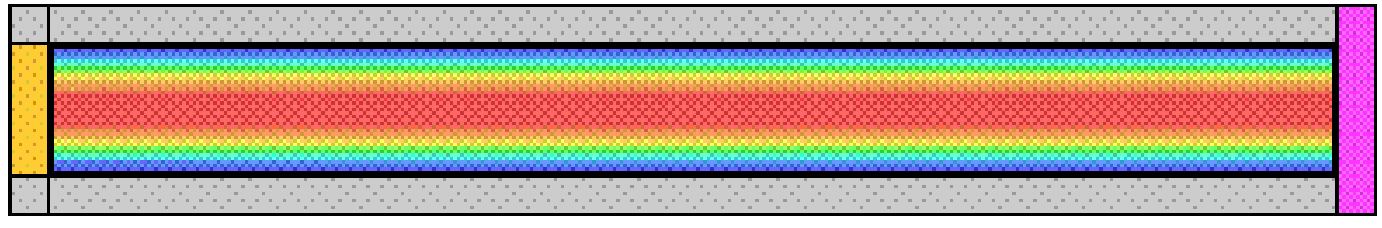

b)
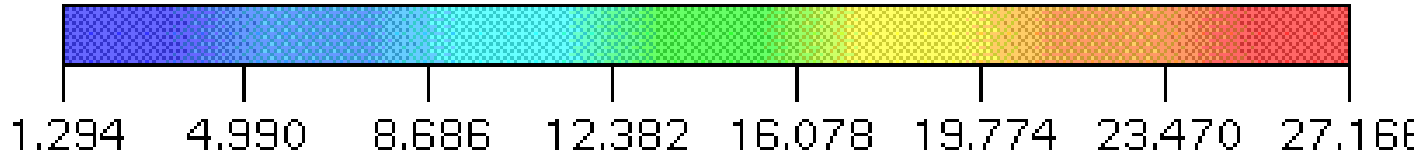

Figura 4.2: Simulação do escoamento em um canal bidimensional. a) Linhas de nível para velocidade $u$ em $t=100$. b) Linhas de nível para $\tau^{x x}$ em $t=100$.

Para demonstrar a convergência do método numérico desenvolvido nesta tese, comparamos as soluções numéricas obtidas nas quatro malhas com a respectiva solução analítica no meio do canal $(x=5 L)$. Os resultados são mostrados nas figuras (4.3), (4.4) e (4.5). Como podemos observar nas figuras (4.3), (4.4) e (4.5), a concordância das soluções numéricas e as respectivas soluções analíticas é excelente: esse fato valida o método numérico desenvolvido neste trabalho para simular escoamentos de fluidos viscoelásticos governados pelo modelo Maxwell. Além disso, podemos observar também que à medida que à malha é refinada, o erro cometido pela solução numérica diminui, o que demonstra a convergência do método numérico. 
a)
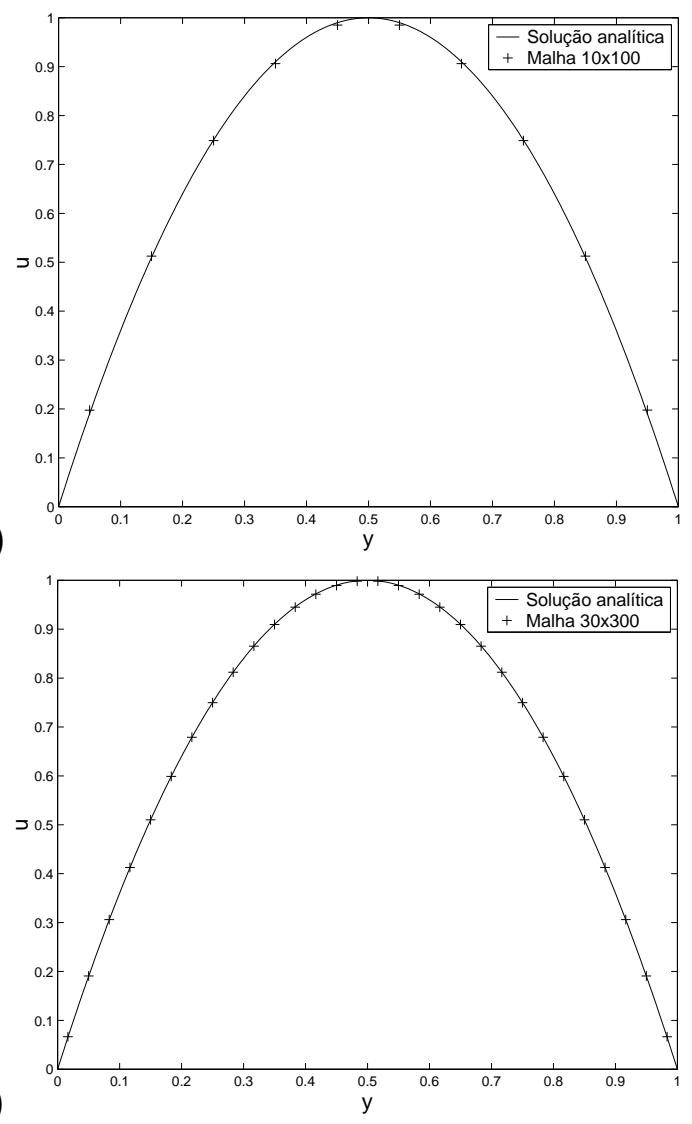

b)

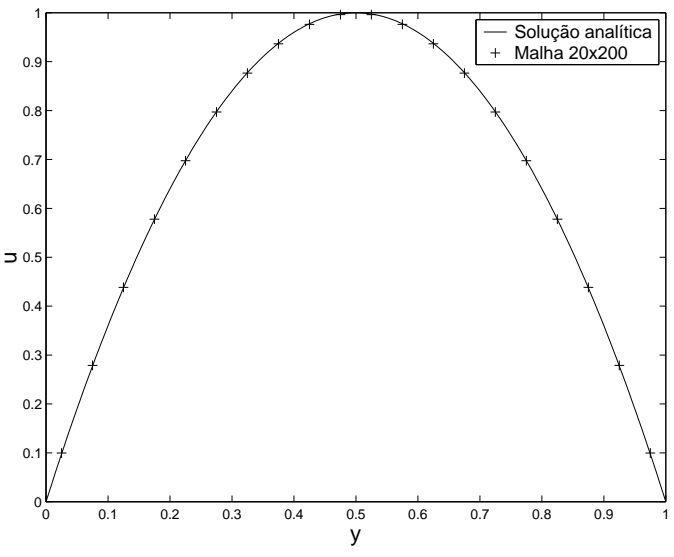

d)

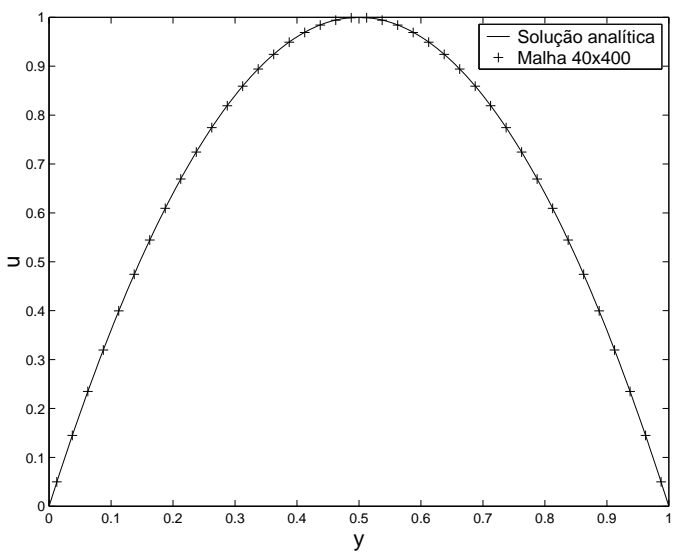

Figura 4.3: Comparação da solução numérica com a solução analítica no meio do canal $(x=5 L)$ : Velocidade $u$. a) Solução obtida na malha M1, b) Solução obtida na malha M2, c) Solução obtida na malha M3, d) Solução obtida na malha M4.

\subsubsection{Modelo K-BKZ}

A técnica numérica apresentada neste trabalho também foi aplicada para simular o escoamento no canal bidimensional usando o modelo integral K-BKZ. Os dados utilizados foram os mesmos usados para a validação do modelo de Maxwell com exceção da definição do fluido. Os parâmetros utilizados foram: $\alpha=500, \beta=0,1, a_{1}=2000, \lambda_{1}=0,005$ s de modo que o $W e=\lambda_{1} \frac{U}{L}=0,5$. Novamente, o código FreeFlow-2D simulou esse problema nas malhas M1, M2 e M3 até que o estado estacionário fosse atingido. Nesse problema não conhecemos a solução analítica mas podemos comparar as soluções obtidas usando as malhas M1 e M2 com a solução obtida na malha mais fina M3. Os resultados obtidos são mostrados nas figuras 4.6 e 4.7 onde podemos observar que à medida que a malha é refinada os resultados aproximam-se da solução numérica obtida na malha M3. Esse fato mostra a convergência do método numérico desenvolvido nesta tese para o modelo 
a)

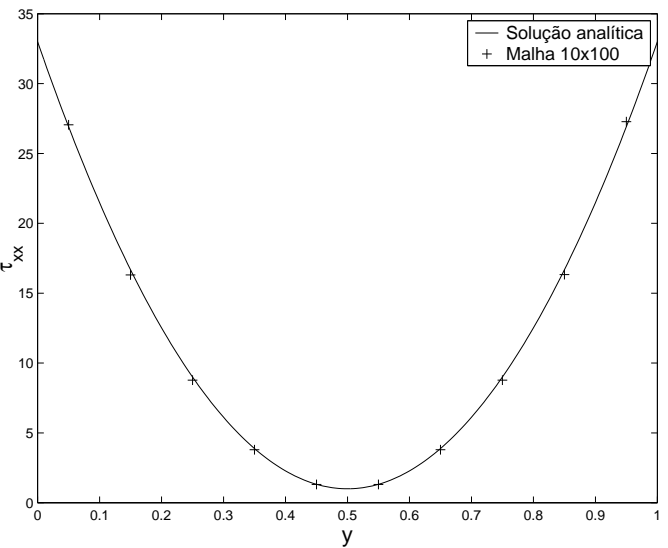

c)

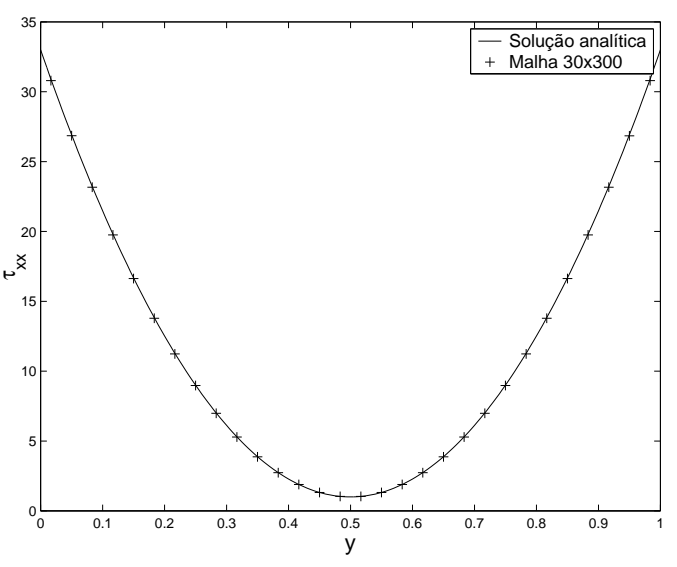

b)

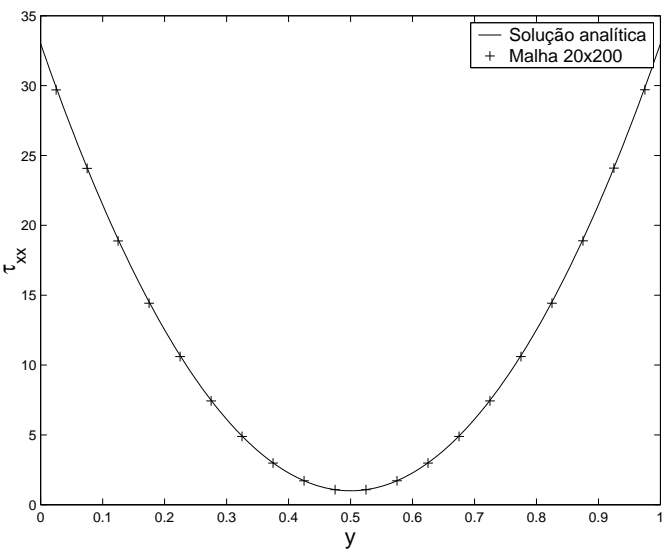

d)

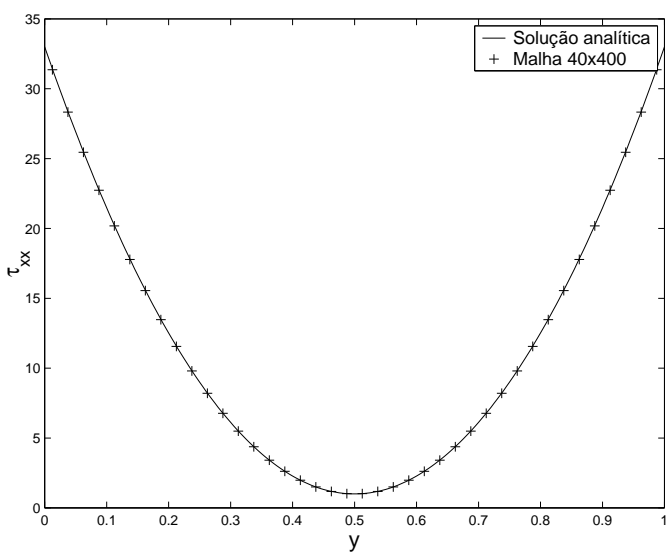

Figura 4.4: Comparação da solução numérica com a solução analítica no meio do canal $(x=5 L)$ : Componente $\tau^{x x}$. a) Solução obtida na malha M1, b) Solução obtida na malha M2, c) Solução obtida na malha M3, d) Solução obtida na malha M4.

integral K-BKZ. 
a)

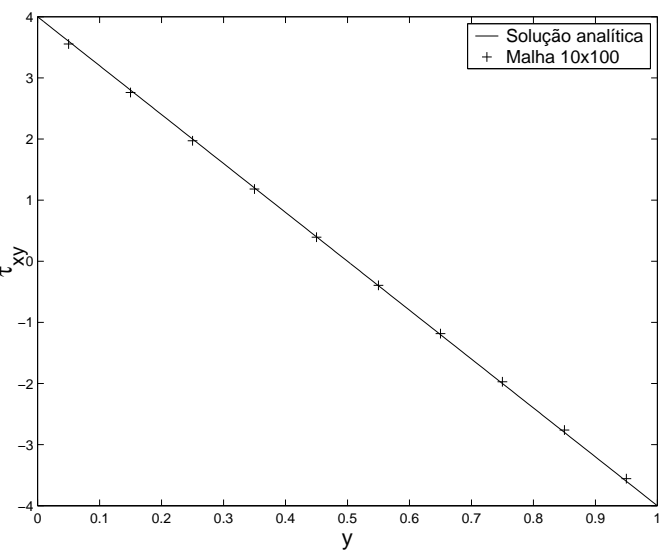

c)

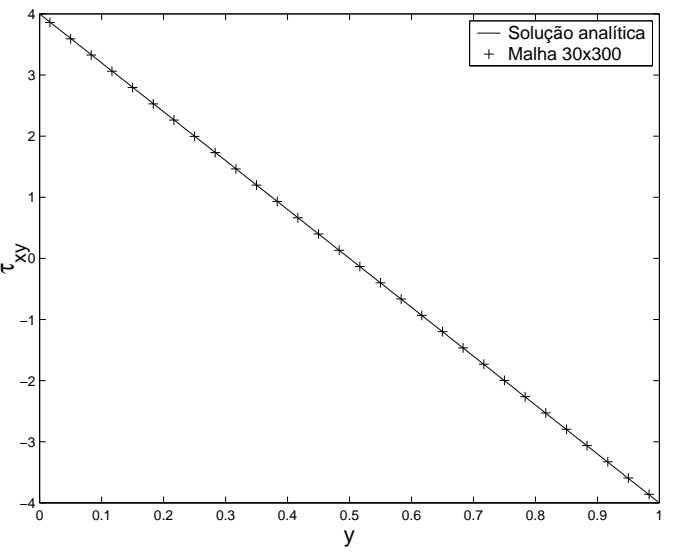

b)

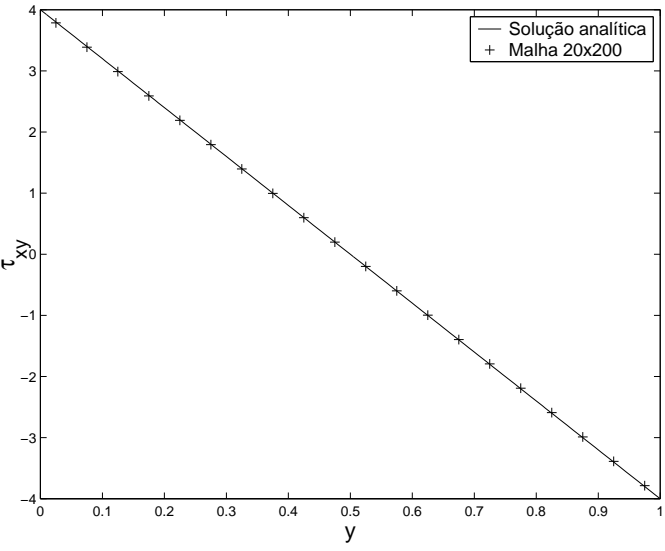

d)

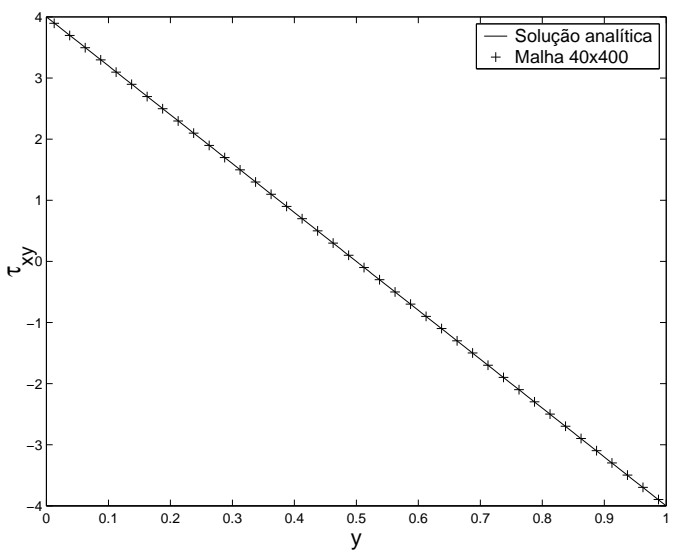

Figura 4.5: Comparação da solução numérica com a solução analítica no meio do canal $(x=5 L)$ : Componente $\tau^{x y}$. a) Solução obtida na malha M1, b) Solução obtida na malha M2, c) Solução obtida na malha M3, d) Solução obtida na malha M4.

\subsubsection{Comparação com resultados experimentais}

Nesta seção é apresentada uma comparação dos resultados obtidos pelo método desenvolvido nesta tese com os obtidos experimentalmente por Quinzani et al. [52]. No trabalho de Quinzani et al. 52 foram medidos os campos de tensão e de velocidade do escoamento de uma solução viscoelástica através de uma contração planar 3,97:1. O fluido utilizado foi uma solução de poliisobutileno em tretradecano (PIB/C14) a 5\%. Este fluido foi reologicamente caracterizado por Quinzani et al. [53] com diversas temperaturas e concentrações, para os regimes permanente e transiente, em cisalhamento simples.

Os resultados apresentados a seguir são provenientes da simulação do escoamento através de uma contração planar 4:1 e as dimensões do domínio utilizado têm como referência o valor $h=0,32 \mathrm{~cm}$ (ver figura 4.25). No modelo utilizado por Quinzani et al. [52], o comprimento de entrada foi de $2 H=2,54 \mathrm{~cm}$ e o utilizado nesta tese foi 


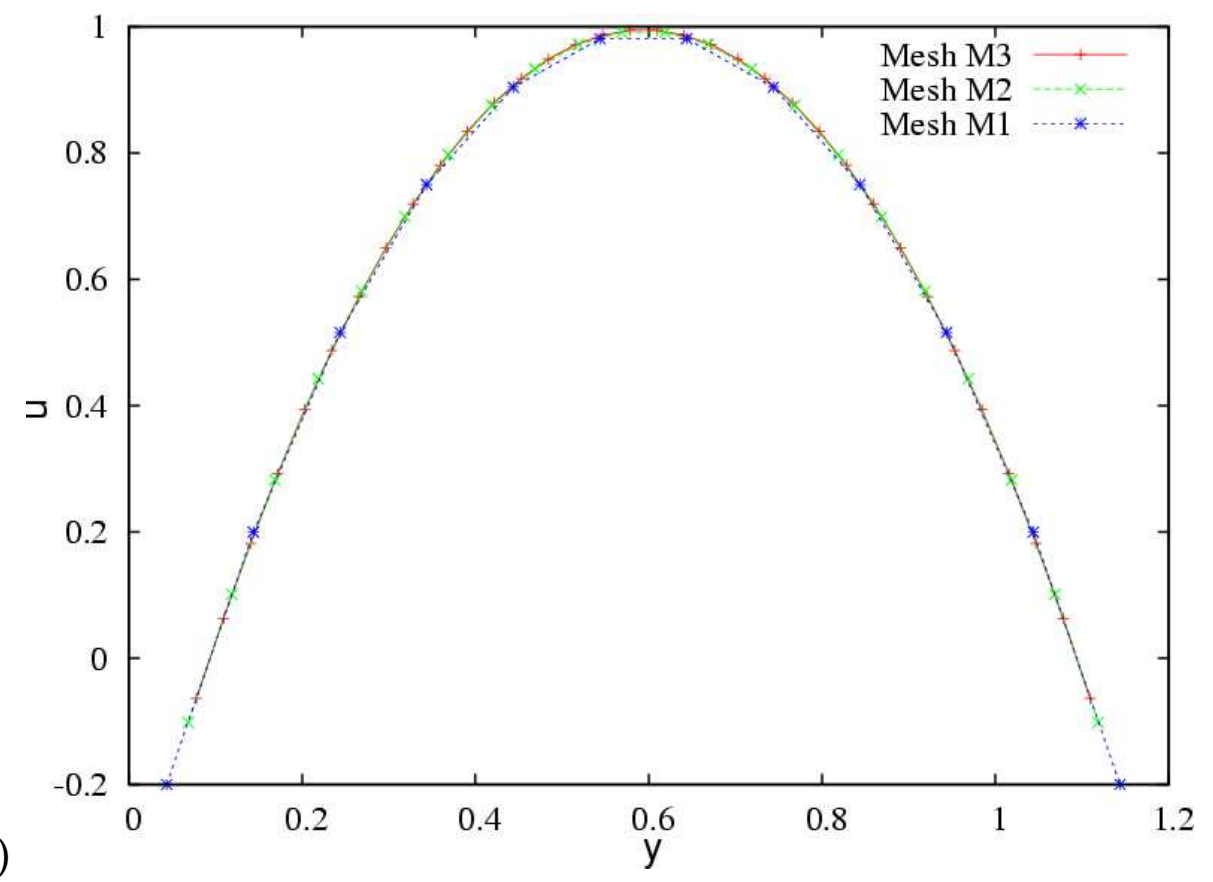

a)

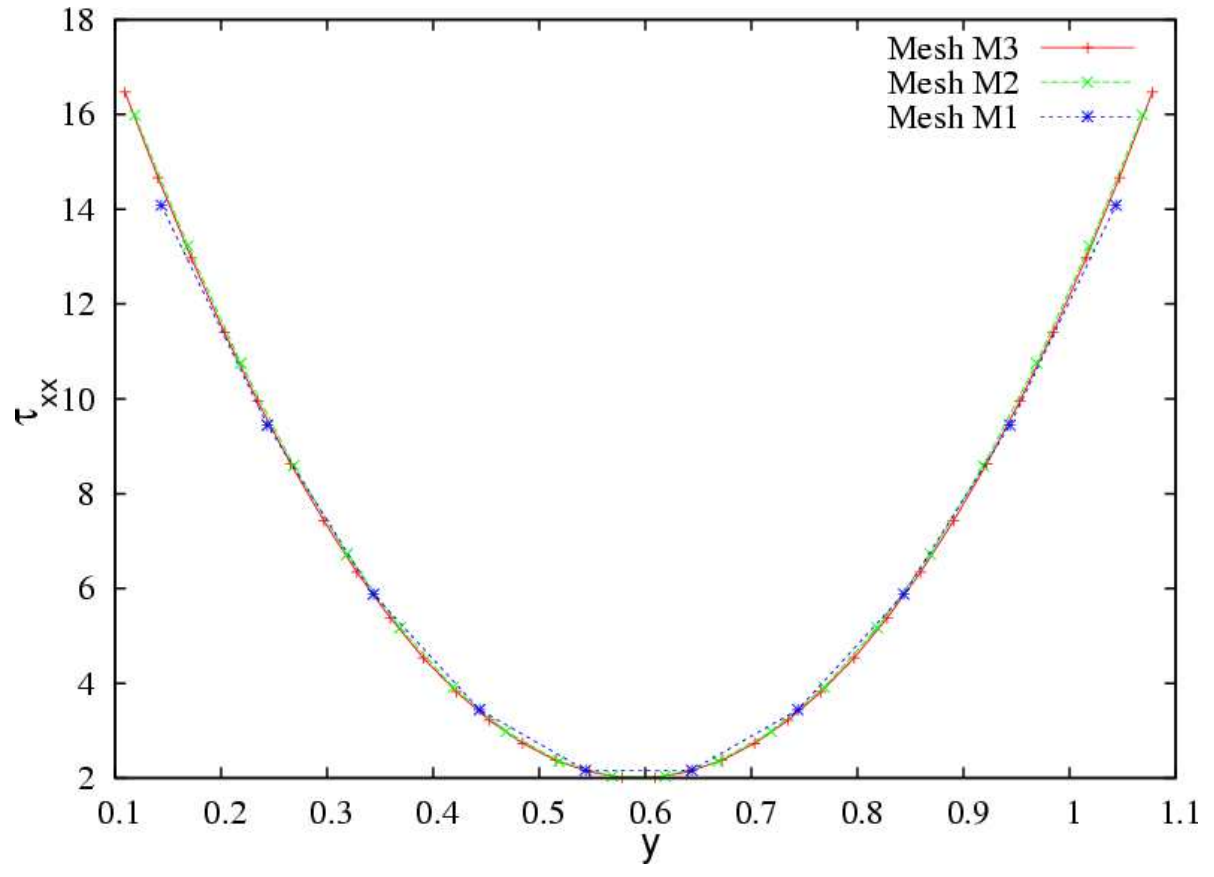

Figura 4.6: Simulação do escoamento em um canal bidimensional usando o modelo KBKZ. Solução numérica obtida no meio do canal $(x=5 L) \operatorname{com} R e=1$ e $W e=0,5$ usando as malhas M1, M2 e M3. a) velocidade $u$, b) tensão $\tau^{x x}$.

$2 H=2,56 \mathrm{~cm}$.

Na caracterização reológica da solução de PIB/C14 feita por Quinzani et al. [52] o espectro de tempos de relaxação foi determinado e esses dados foram utilizados para testar os modelos reológicos Oldroyd-B, Giesekus e Bird-DeAguiar para ajustar os dados 


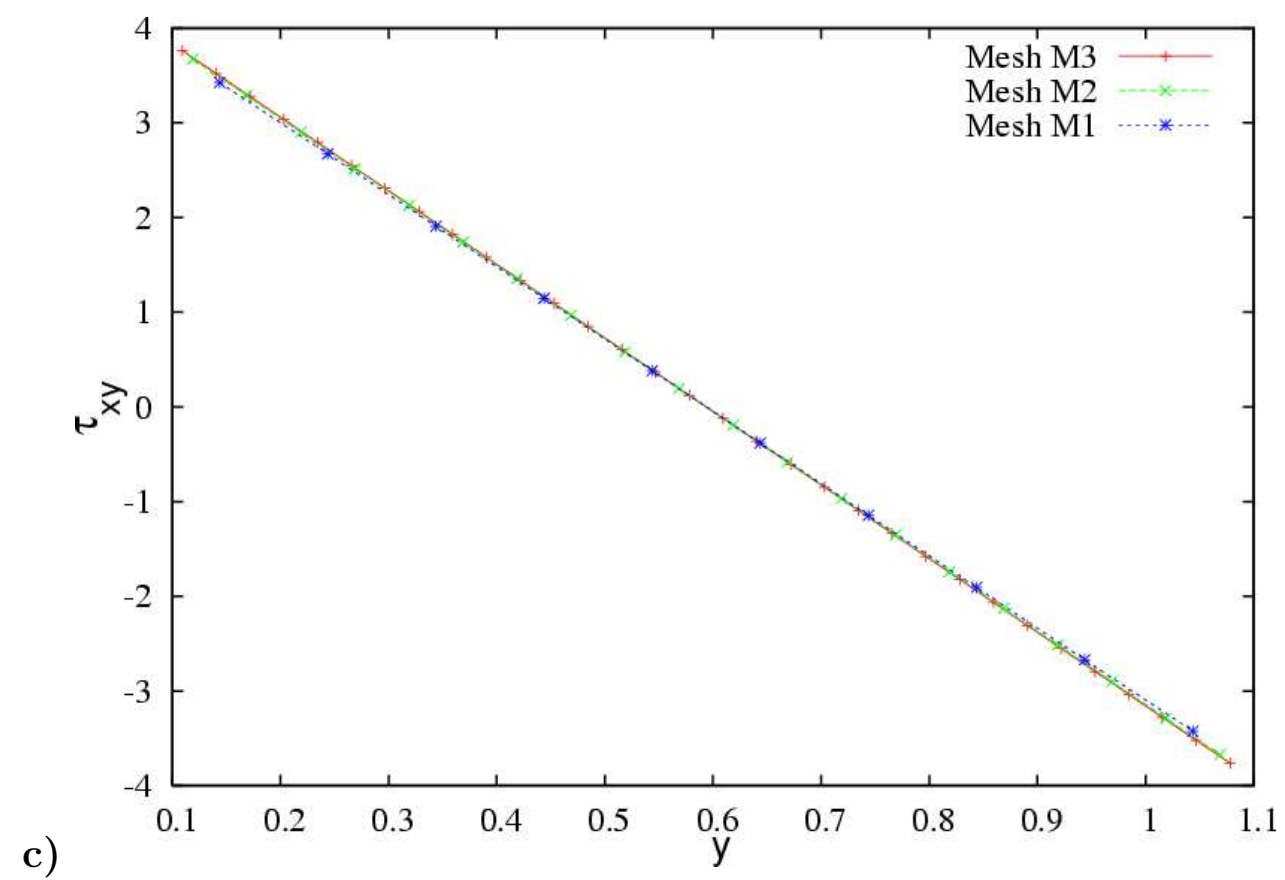

c)

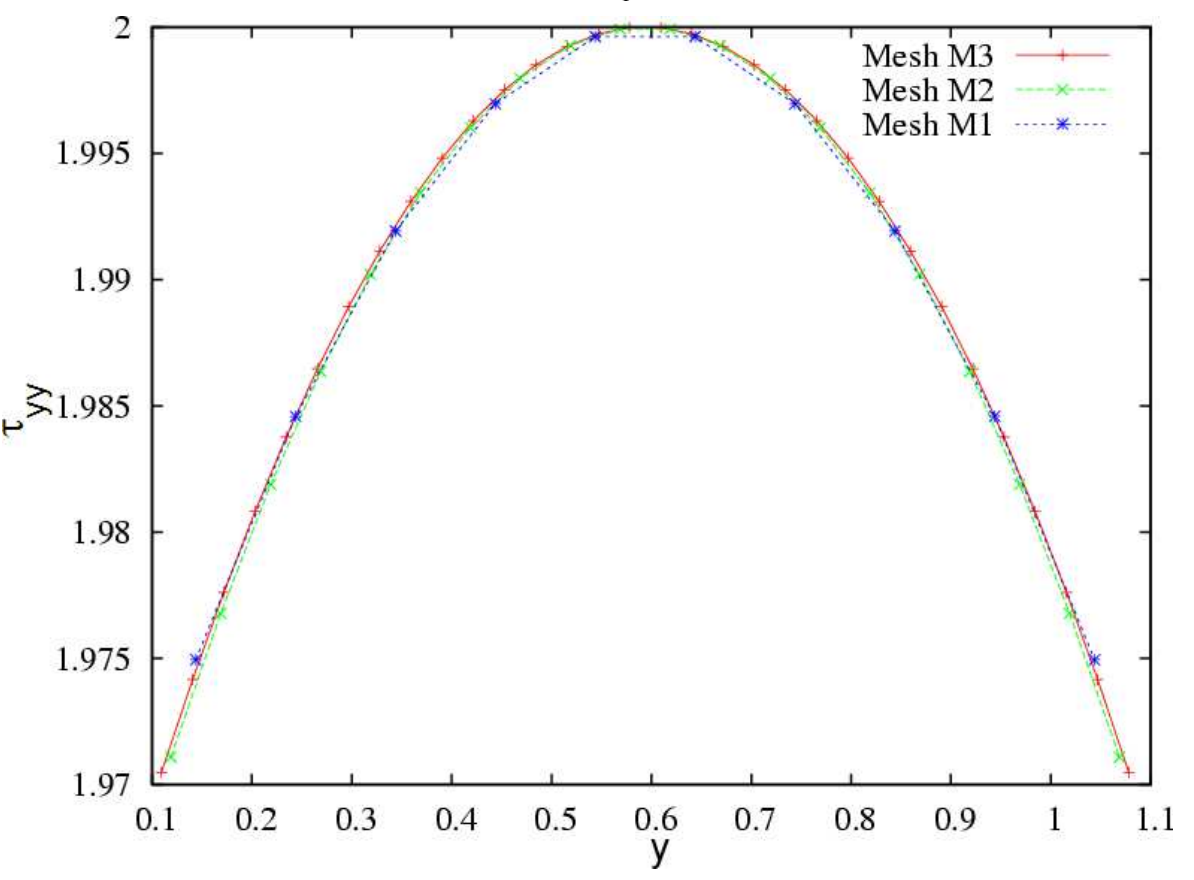

Figura 4.7: Simulação do escoamento em um canal bidimensional usando o modelo KBKZ. Solução numérica obtida no meio do canal $(x=5 L)$ com $R e=1$ e $W e=0,5$ usando as malhas M1, M2 e M3. c) tensão $\left.\tau^{x y}, \mathbf{d}\right)$ tensão $\tau^{y y}$.

experimentais. Posteriormente, Azaiez et al. [5] usou a mesma solução para validar o uso de um método numérico com os modelos Giesekus, FENE-P e PTT, na simulação do escoamento através da contração planar 4:1, obtendo concordância qualitativa entre os resultados numéricos e experimentais. Os parâmetros $\alpha$ e $\beta$ utilizados na equação K-BKZ 
foram obtidos por Mitsoulis [35] e são dados por $\alpha=10$ e $\beta=0,7$. O espectro de tempos de relaxação é mostrado na tabela 4.1, onde os parâmetros $a_{k}$ foram obtidos pela relação $a_{k}=\frac{\eta_{k}}{\lambda_{k}}$.

Utilizando os valores de $\eta_{0}, \lambda_{k}, a_{k}$ (ver tabela 4.1) e os valores de $\alpha, \beta$ apresentados por Mitsoulis [35], empregamos o método numérico descrito no capítulo 2 para calcular as funções materiais $\eta=\frac{\tau^{x y}}{\dot{\gamma}}$ e $\Psi_{1}=\frac{\tau^{x x}-\tau^{y y}}{\dot{\gamma}^{2}}$ em função da taxa de cisalhamento $\dot{\gamma}$. Os resultados obtidos são mostrados na figura 4.8, onde podemos observar que os resultados numéricos mostram excelente concordância com os dados experimentais apresentados por Quinzani et al. [53]. Além disso, as funções materiais $\eta^{+}(t, \dot{\gamma})=\frac{\tau^{x y}(t)}{\dot{\gamma}} \mathrm{e}$ $\Psi_{1}^{+}(t, \dot{\gamma})=\frac{\tau^{x x}(t)-\tau^{y y}(t)}{\dot{\gamma}^{2}}$ também foram calculadas e os resultados são mostrados na figura 4.9. Podemos observar que os dados mostrados na figura 4.9 mostram uma boa concordância com os dados experimentais, especialmente para tempos maiores e para altas taxas de cisalhamento.

Tabela 4.1: Parâmetros da equação K-BKZ para um fluido estudado por Quinzani et al. [53]. $\alpha=10, \beta=0,7[35], \eta_{0}=1,424 \mathrm{~Pa} \mathrm{~s}, \lambda_{0}=0,06 \mathrm{~s}$

\begin{tabular}{cccc}
\hline \hline$k$ & $\lambda_{k}(\mathrm{~s})$ & $\eta_{k}(\mathrm{~Pa} \mathrm{~s})$ & $a_{k}(\mathrm{~Pa})$ \\
\hline 1 & 0,6855 & 0,0400 & 0,058352 \\
2 & 0,1396 & 0,2324 & 1,664756 \\
3 & 0,0389 & 0,5664 & 14,560411 \\
4 & 0,0059 & 0,5850 & 99,152542 \\
\hline \hline
\end{tabular}

Com o objetivo de comparar os resultados numéricos obtidos nesta tese com os apresentados por Quinzani et al. [52], as tensões e velocidades serão apresentadas na forma dimensional. Devido a convenção de sinais para a tensão ser oposta no artigo de Quinzani et al. [52]. os sinais dos resultados apresentados também foram ajustados. Além disso, usaremos as definições de número de Reynolds e de número de Deborah usadas por Quinzani et al. [52].

$$
\operatorname{Re}=\frac{2 h \rho<v>}{\eta_{0}}
$$

e

$$
\text { De }=\lambda_{0} \dot{\gamma}
$$




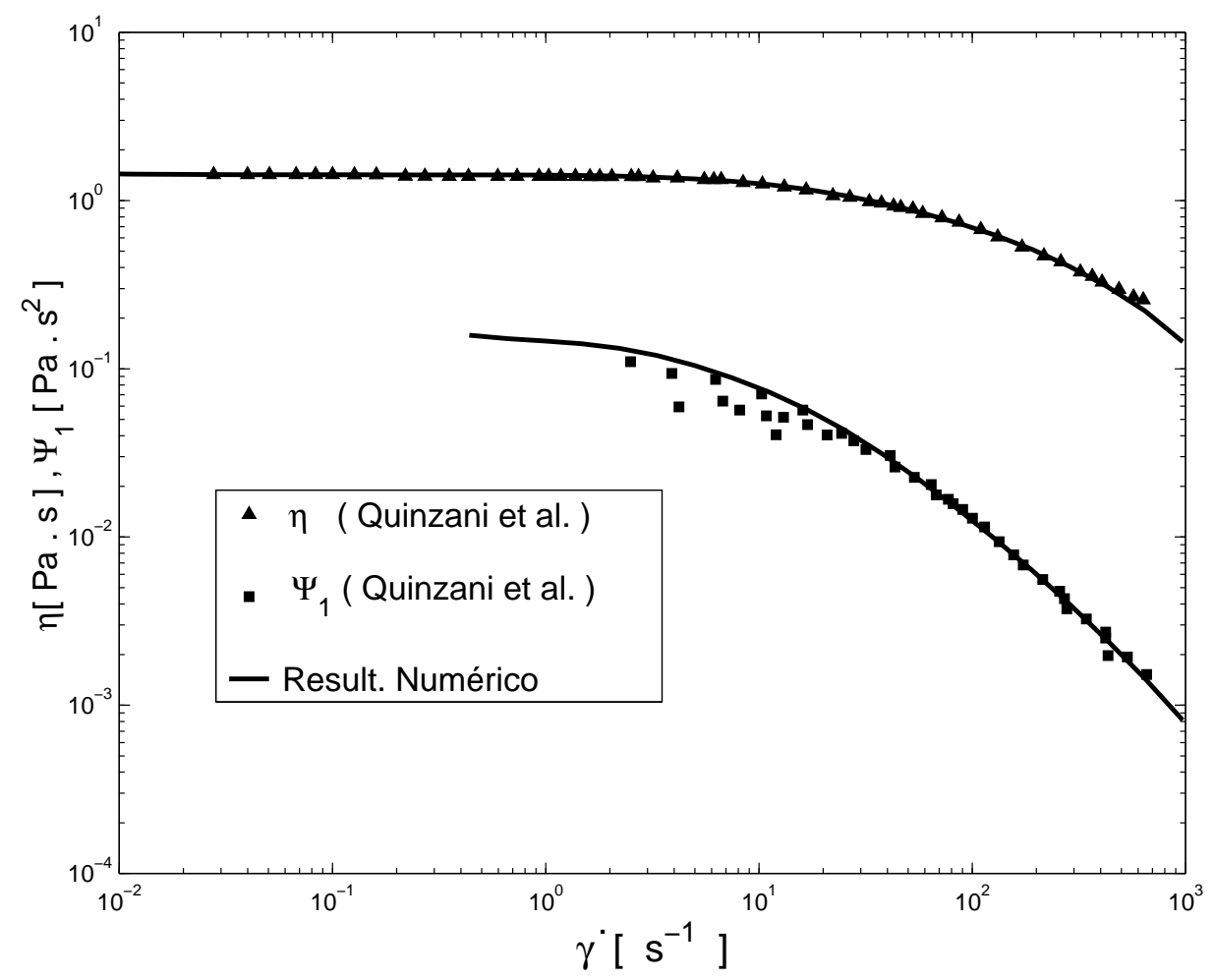

Figura 4.8: Resultados numéricos obtidos para $\eta=\frac{\tau^{x y}}{\dot{\gamma}}$ e $\Psi_{1}=\frac{\tau^{x x}-\tau^{y y}}{\dot{\gamma}^{2}}$ e comparação com os dados experimentais de Quinzani et al. [53]. Dados utilizados da tabela 4.1.
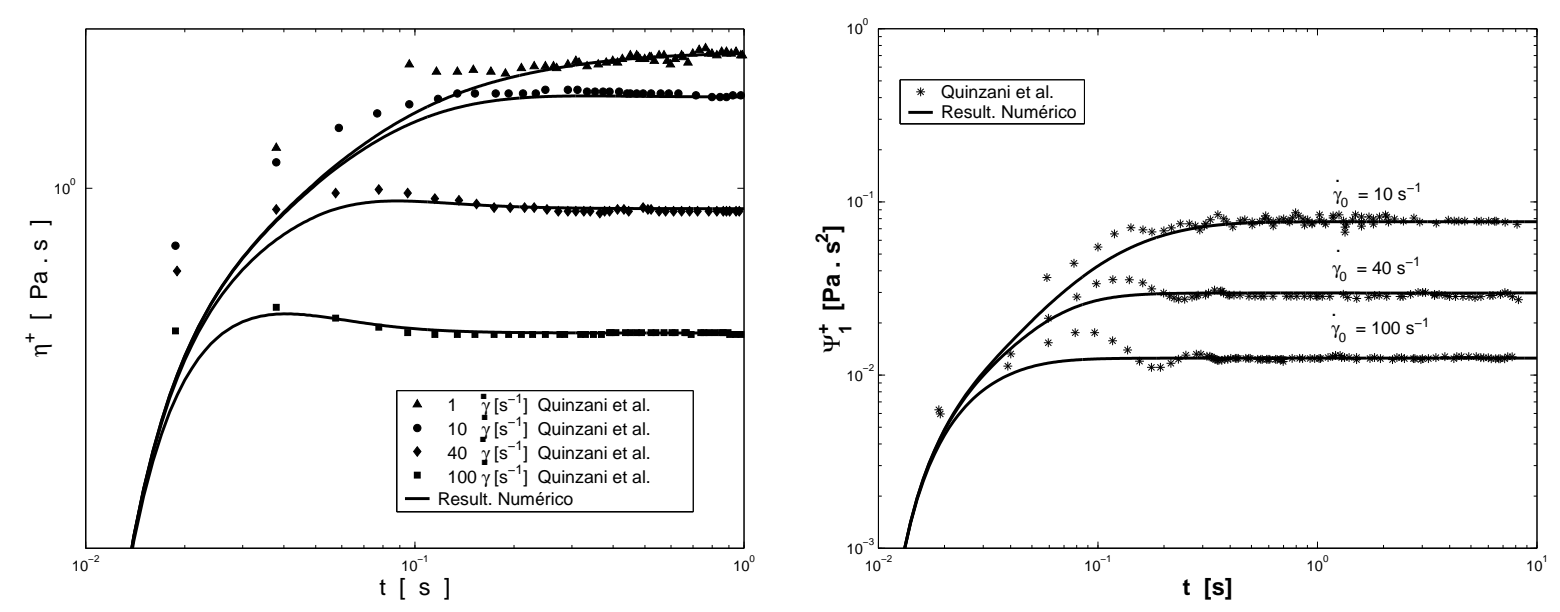

Figura 4.9: Resultados obtidos para $\eta^{+}(t, \dot{\gamma})=\frac{\tau^{x y}(t)}{\dot{\gamma}}$ e $\Psi_{1}^{+}(t, \dot{\gamma})=\frac{\tau^{x x}(t)-\tau^{y y}(t)}{\dot{\gamma}^{2}}$ e comparação com os dados experimentais de Quinzani et al. [53]. Dados utilizados da tabela 4.1 .

onde $\dot{\gamma}=\frac{<v>}{h}$ e $<v>$ é a velocidade média no canal menor.

Quinzani et al. 52] realizou seis experimentos variando a vazão $Q$ e mediu a velocidade e a tensão em vários pontos da contração planar. O método numérico desenvolvido nesta tese utilizou os dados da tabela 4.1 e simulou o escoamento através da contração planar 
com os dados dos experimentos 3 e 5 de Quinzani et al. [52], cujos números de Reynolds e de Deborah são $R e=0,27$ e $D e=1,39$ para o experimento 3 e $R e=0,56$ com $D e=2,9$ para o experimento 5. As velocidades médias no canal menor são $\langle v>=7,44 \mathrm{~cm} / \mathrm{s}$ para o experimento 3 e $\langle v\rangle=15,5 \mathrm{~cm} / \mathrm{s}$ para o experimento 5 . O perfil parabólico de velocidade imposto na entrada de fluido foi calculado de modo a obter a mesma vazão no canal maior.

Uma vez conhecida a velocidade média no canal maior, o perfil parabólico imposto na entrada de fluido foi dado por

$$
u(y)=-\frac{4 U_{i n f}}{(2 H)^{2}}\left(y-\frac{(2 H)}{2}\right)^{2}+U_{i n f},
$$

onde $U_{\text {inf }}$ é a velocidade máxima. A relação entre $U_{\text {inf }}$ e $\langle v\rangle_{\text {inf }}$ (velocidade média na entrada) é obtida por meio da equação

$$
<v>_{\text {inf }}=\frac{1}{2 H} \int_{0}^{2 H} u(y) d y
$$

e é dada por

$$
U_{\text {inf }}=\frac{3}{2}<v>_{\text {inf }}
$$

Os resultados obtidos na simulação com $D e=2,9$ foram comparados com os de Quinzani et al. [52] e são mostrados nas figuras (4.10)-(4.16).

As figuras $4.10 \mathrm{~b}$ e $4.11 \mathrm{~b}$ mostram os resultados obtidos numericamente para a componente $u$ da velocidade em perfis axiais e transversais a montante da entrada da contração. Valores obtidos experimentalmente por Quinzani et al. [52] também são mostrados para comparação nas figuras 4.10a e 4.11a. Pode ser observado a evolução a partir do perfil parabólico totalmente desenvolvido para perfis cuja velocidade aumenta à medida que o fluxo se aproxima da entrada. A concordância com os dados experimentais é melhor para pontos mais afastados do plano de contração (ver figura 4.10). Na figura 4.11 podese observar que os resultados numéricos ajustam-se melhor aos dados experimentais em direções axiais do que em direções transversais. Este fato também foi relatado por Azaiez et al. [5] na simulação desse problema usando o modelo Giesekus.

Podemos observar na figura 4.12 que a componente cisalhante $\tau_{x y}$ cresce de zero até um valor máximo, que aumenta com a proximidade da entrada da contração. Como ocorreu com a velocidade, a concordância é melhor à medida que nos afastamos da entrada da contração, o mesmo ocorrendo para os perfis axiais como mostrado na figura 4.13. Com 
a)

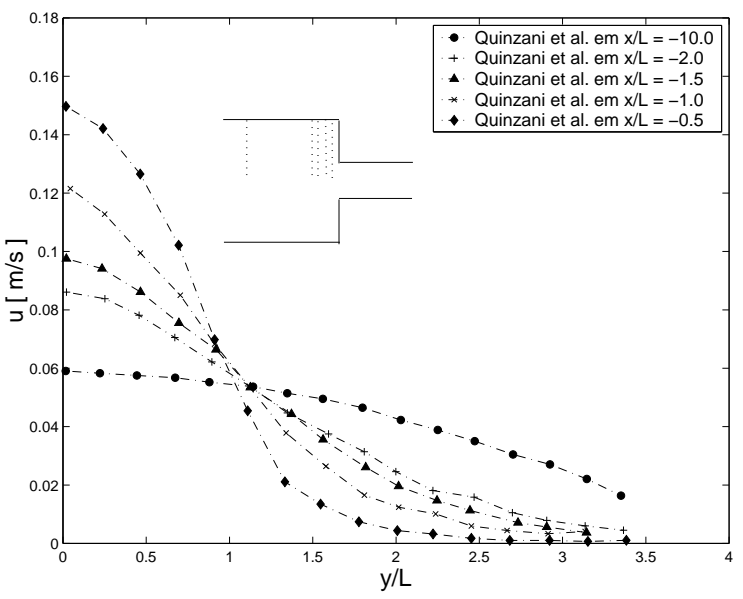

b)

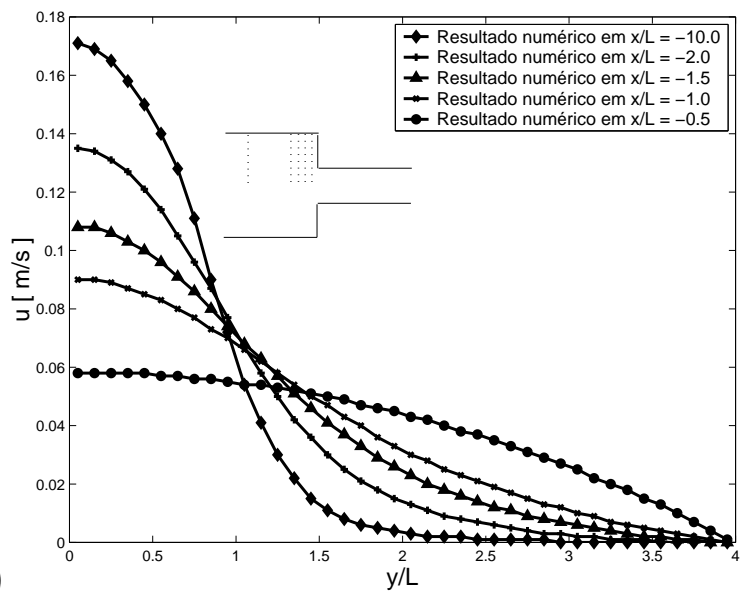

Figura 4.10: Simulação numérica do escoamento em uma contração com os dados de Quinzani et al. [52] $(R e=0,56$ e $D e=2,9)$. a) Valores obtidos experimentalmente para a velocidade $u(y)$ para diversos valores de $x / L$. b) Valores obtidos numericamente nos mesmos pontos.

a)

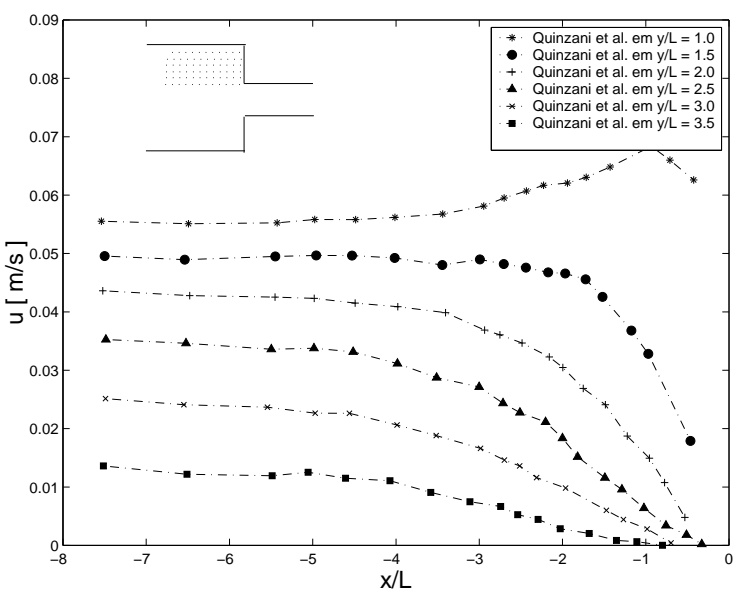

b)

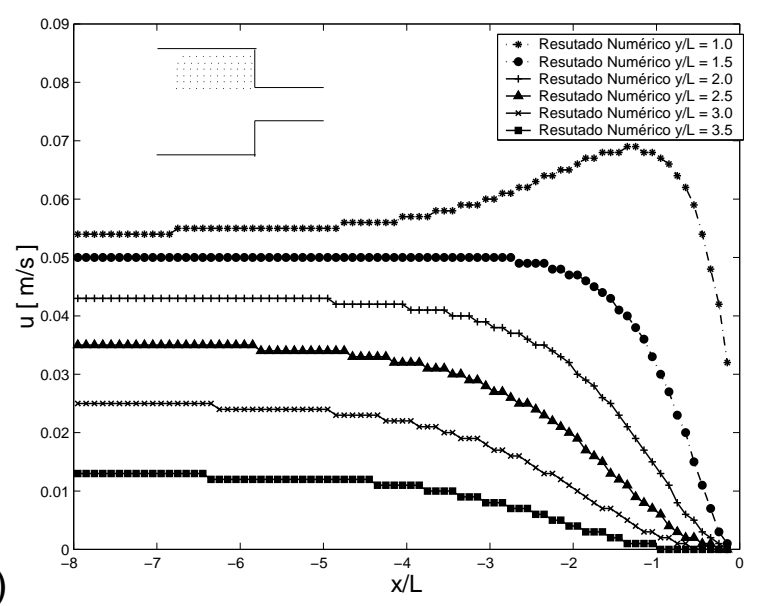

Figura 4.11: Simulação numérica do escoamento em uma contração com os dados de Quinzani et al. [52] ( $R e=0.56$ e $D e=2,9)$. a) Valores obtidos experimentalmente para a velocidade $u(x)$ para diversos valores de $y / L$. b) Valores obtidos numericamente nos mesmos pontos.

relação a figura 4.14, podemos observar que em perfis transversais a primeira diferença de tensões normais cresce na região central com a proximidade do plano de contração e são menores próximo aos contornos devido à desaceleração do fluido nessa região. Por outro lado, a figura 4.15 mostra que a diferença entre os resultados numéricos e os resultados experimentais cresce com a proximidade do eixo de simetria. De um modo geral, as figuras 4.14 e 4.15 mostram que os resultados numéricos estão em boa concordância com 
os resultados experimentais.

a)

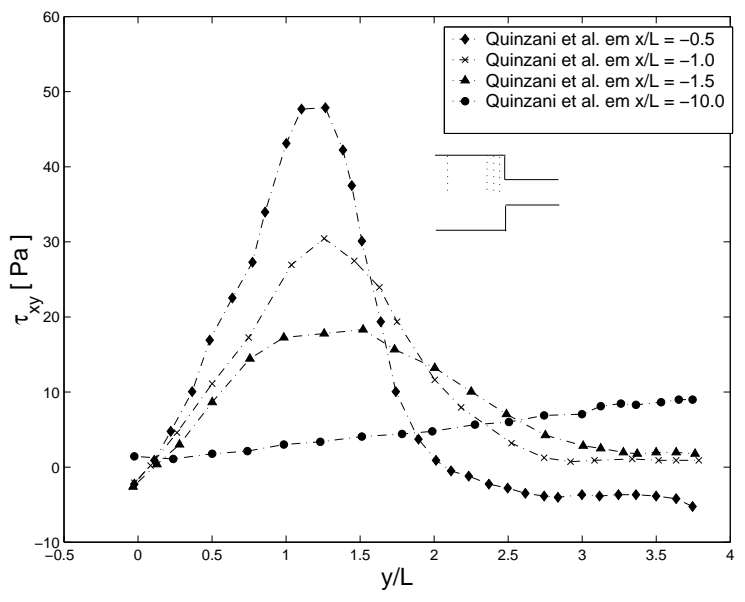

b)

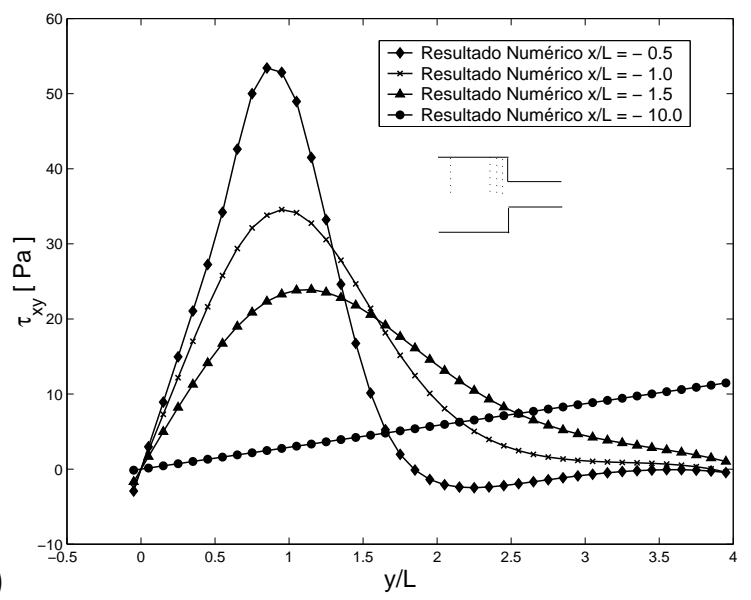

Figura 4.12: Simulação numérica do escoamento em uma contração com os dados de Quinzani et al. [52] ( $R e=0.56$ e $D e=2,9)$. a) Valores obtidos experimentalmente para a componente $\tau^{x y}(y)$ para diversos valores de $x / L$. b) Valores obtidos numericamente nos mesmos pontos.

a)

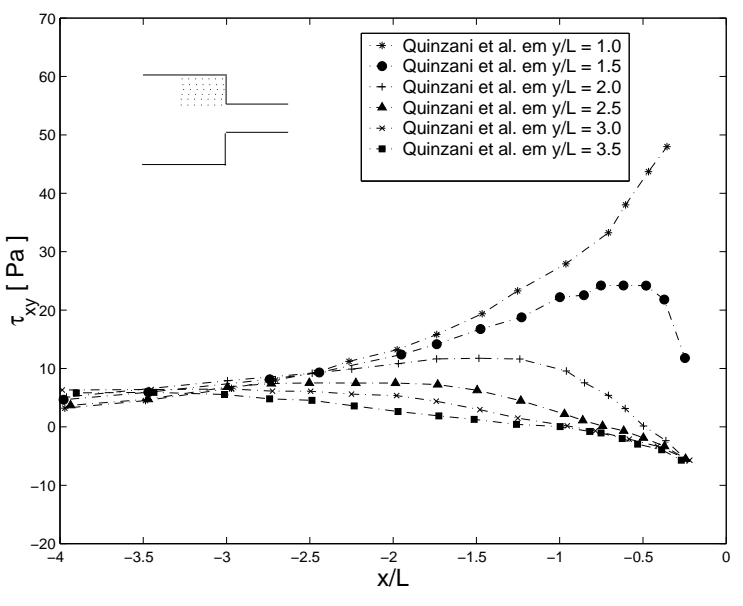

b)

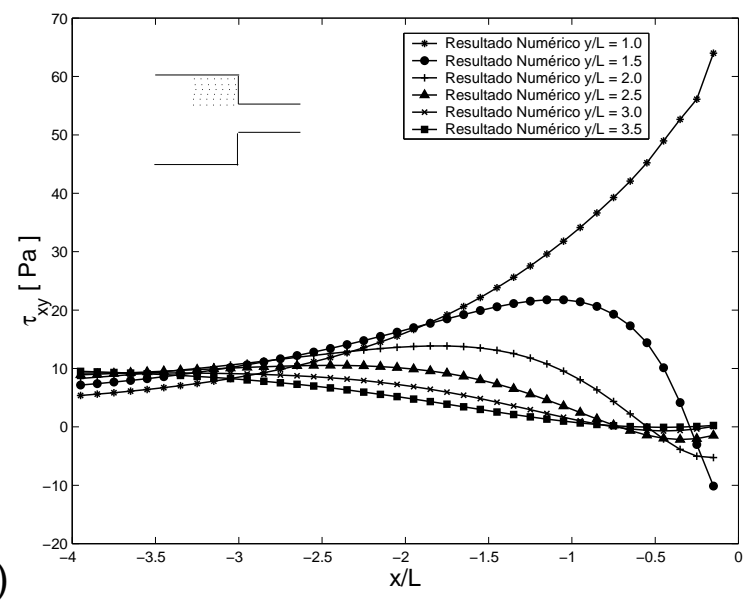

Figura 4.13: Simulação numérica do escoamento em uma contração com os dados de Quinzani et al. [52] ( $R e=0,56$ e $D e=2,9)$. a) Valores obtidos experimentalmente para a componente $\tau^{x y}(x)$ para diversos valores de $y / L$. b) Valores obtidos numericamente nos mesmos pontos.

As figuras 4.16, 4.18 e 4.19, apresentam os perfis da velocidade $(u(y))$, primeira diferença de tensões normais $(N 1)$ e tensão de cisalhamento $\left(\tau^{x y}\right)$ obtidos no canal menor para vários valores de $x / L$. Podemos observar que os perfis da tensão de cisalhamento e os perfis da primeira diferença de tensões normais apresentam uma boa concordância 
a)

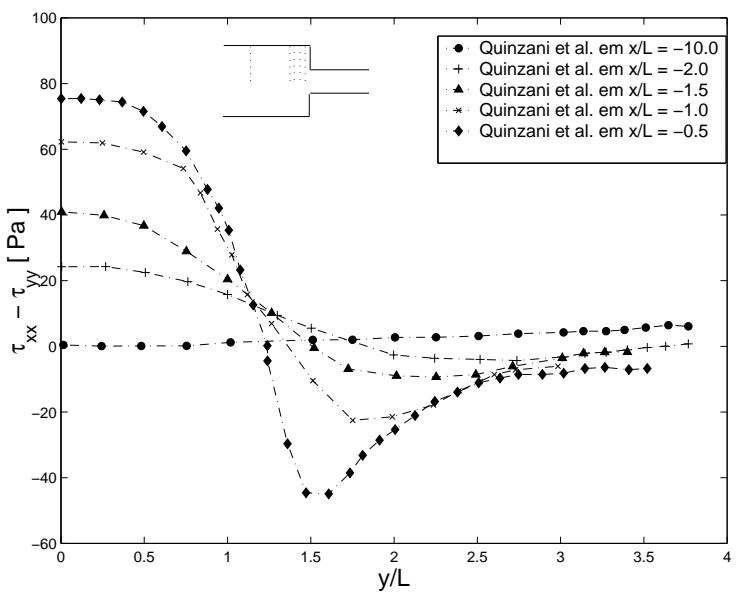

b)

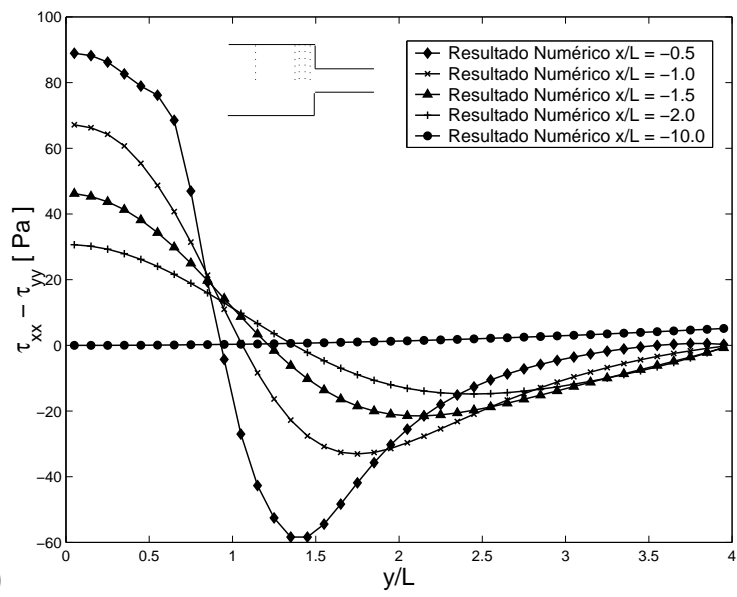

Figura 4.14: Simulação numérica do escoamento em uma contração com os dados de Quinzani et al. [52] $(R e=0,56$ e $D e=2,9)$. a) Valores obtidos experimentalmente para a primeira diferença de tensões normais $N 1(y)$ para diversos valores e $x / L$. b) Valores obtidos numericamente nos mesmos pontos.

a)

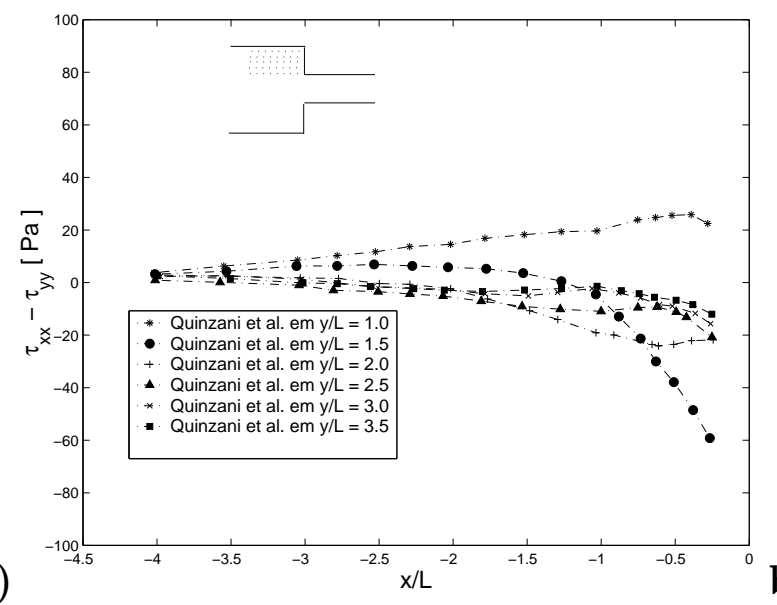

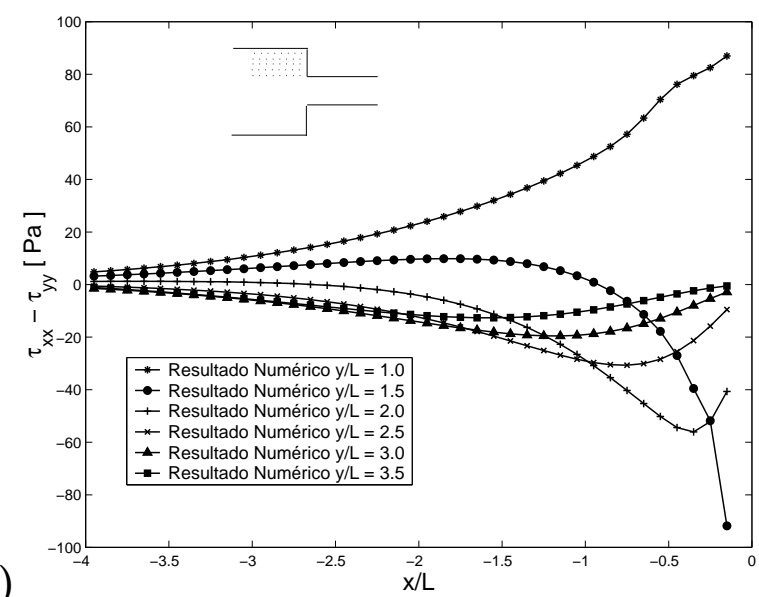

Figura 4.15: Simulação numérica do escoamento em uma contração com os dados de Quinzani et al. [52] $(R e=0,56$ e $D e=2,9)$. a) Valores obtidos experimentalmente para a primeira diferença de tensões normais $N 1(x)$ para diversos valores e $y / L$. b) Valores obtidos numericamente nos mesmos pontos.

com os dados experimentais de Quinzani et al. [52], com uma mudança gradual na forma da primeira diferença de tensões normais de côncava para convexa e com o crescimento da tensão de cisalhamento para pontos mais próximos à parede. Por outro lado, podemos observar na figura 4.16a que o perfil de velocidade apresenta valores maiores que os encontrados experimentalmente e portanto não apresenta uma boa concordância com os dados experimentais. Nos resultados numéricos houve um salto da velocidade na entrada 
da contração, o que fez com que a velocidade máxima próximo à entrada seja maior do que em pontos mais afastados. Esse salto pode ser visto na figura 4.17 a, onde é mostrado a velocidade $u$ no eixo de simetria, juntamente com o resultado experimental de Quinzani et al. Neste mesmo gráfico também é mostrado o resultado numérico obtido por Azaiez et al. [5], onde também pode ser observado um salto no valor da velocidade após a entrada na contração. A figura 4.17b apresenta os resultados para a primeira diferença de tensões normais ao longo do eixo de simetria, onde podemos observar uma boa concordância com os valores experimentais na região próxima da entrada da contração.

A figura 4.20 mostra os resultados obtidos para a componente $\tau^{x y}(x)$ para os valores $y / L=0,0,0,4,1,0$. Podemos observar que para os valores de $y / L=0,0$ e $y / L=1,0$, os resultados numéricos apresentam uma boa concordância com os resultados experimentais de Quinzani et al. [52]. Porém, para $y / L=0,4$ os resultados numéricos não apresentam uma boa concordância devido a uma oscilação da tensão de cisalhamento na entrada da contração. Esse fato também foi obtido nos resultados numéricos apresentados por Azaiez et al. [5] e os resultados experimentais com $D e=1,39$ mostram essa oscilação nos valores de $\tau^{x y}$ para $y / L=0,5$ (ver figura 4.24)

a)

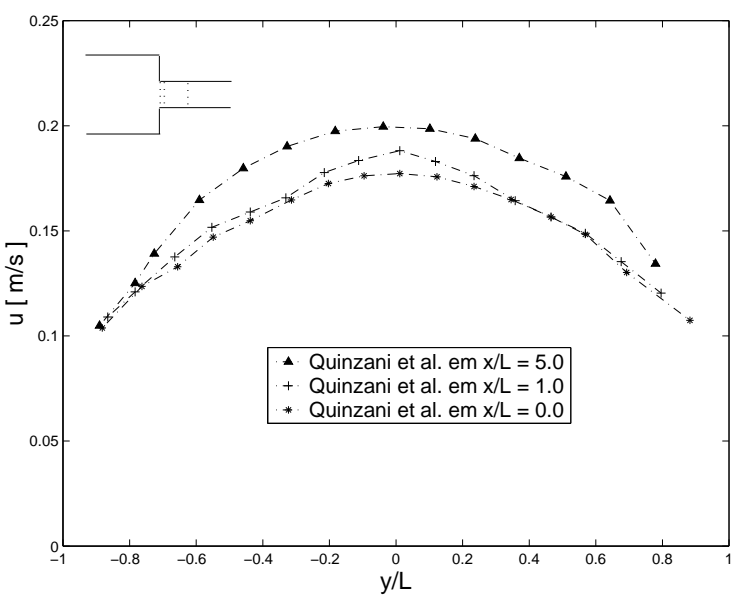

b)

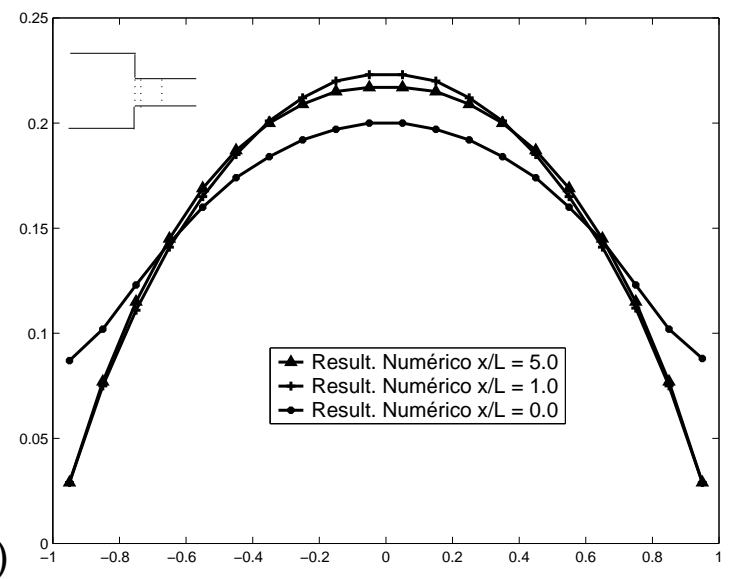

Figura 4.16: Simulação numérica do escoamento em uma contração com os dados de Quinzani et al. [52] $(R e=0,56$ e $D e=2,9)$. a) Valores obtidos experimentalmente para a velocidade $u(y)$ para diversos valores de $x / L$ no canal menor. b) Valores obtidos numericamente nos mesmos pontos.

O código FreeFlow-2D também simulou o escoamento através da contração planar 4:1 com os dados utilizados no experimento 3 de Quinzani et al. [52], cujo número de Reynolds e número de Deborah são $R e=0,27$ e $D e=1,39$. Os resultados obtidos foram 
a)

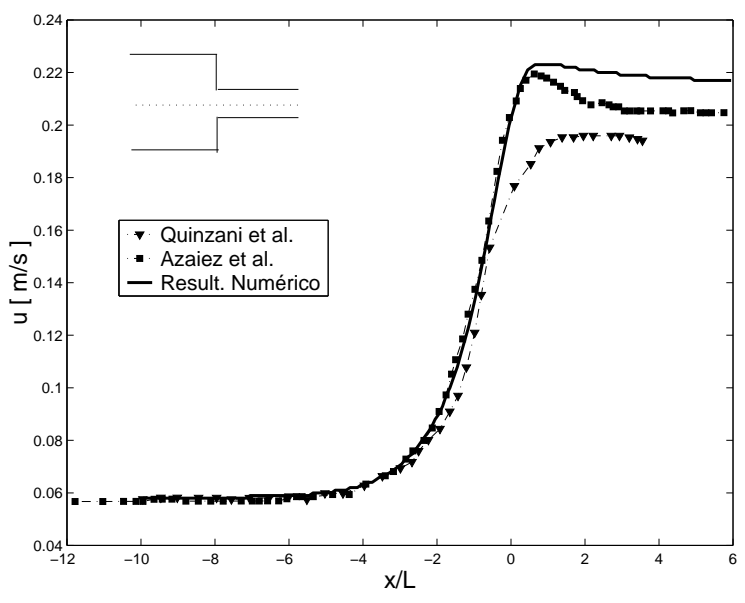

b)

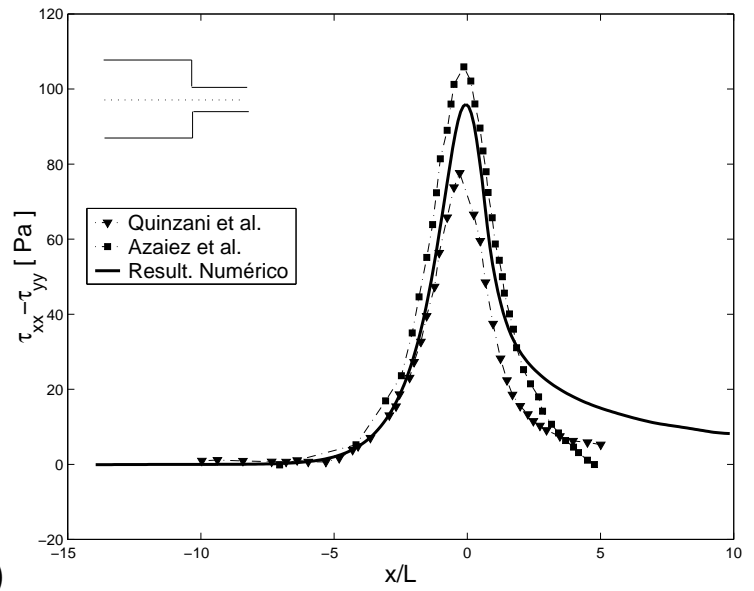

Figura 4.17: Simulação numérica do escoamento em uma contração com os dados de Quinzani et al. [52] $(R e=0,56$ e $D e=2,9)$. a) Valores obtidos experimentalmente para a velocidade $u$ ao longo do eixo de simetria: ( $)$ Quinzani et al. [52]; ( $)$ Azaiez et al. [5], (-) FreeFlow2D. b) Valores obtidos numericamente para a primeira diferença de tensões normais $N 1$ nos mesmos pontos: ( $)$ Quinzani et al. [52]; ( $)$ Azaiez et al. [5], ( -) FreeFlow2D.

a)

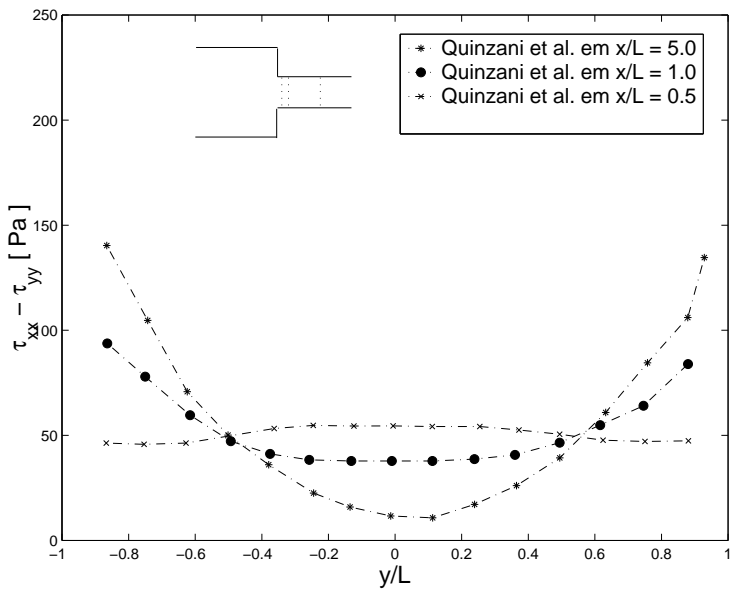

b)

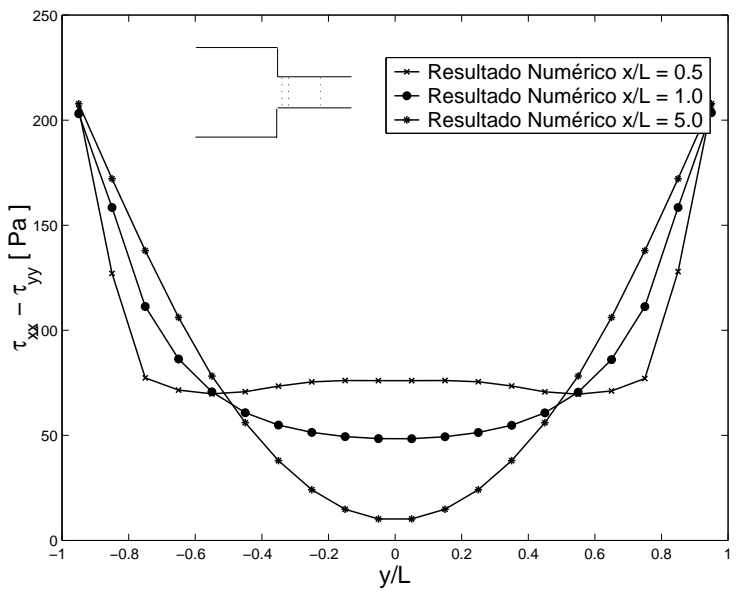

Figura 4.18: Simulação numérica do escoamento em uma contração com os dados de Quinzani et al. [52] $(R e=0,56$ e $D e=2,9)$. a) Valores obtidos experimentalmente para a primeira diferença de tensões normais $N 1(y)$ para diversos valores e $x / L$ no canal menor. b) Valores obtidos numericamente nos mesmos pontos.

semelhantes àqueles apresentados para o experimento $5(R e=0,56$ e $D e=2,9)$. Alguns resultados obtidos nessa simulação são mostrados nas figuras 4.21 - 4.24 .

Na figura 4.21 observa-se que os perfis verticais de velocidade a montante da entrada da contração apresentaram melhor concordância que o caso anterior. Também os resultados 
a)

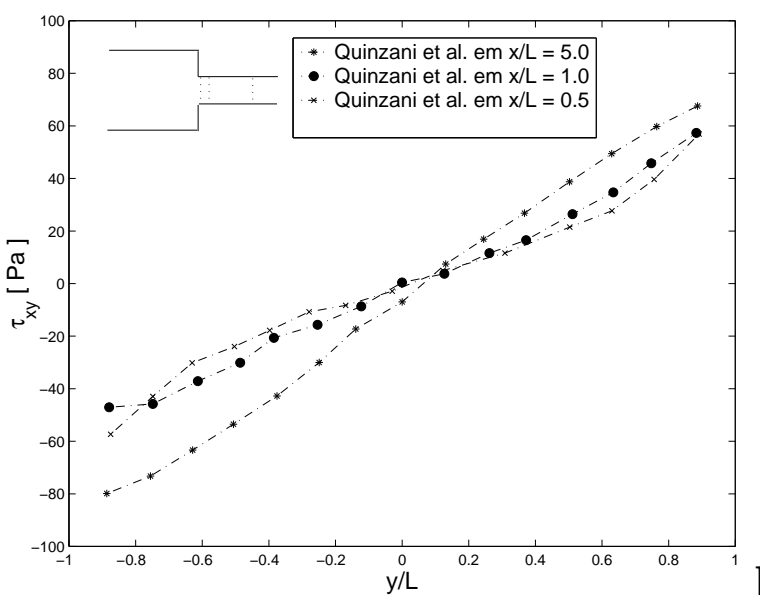

b)

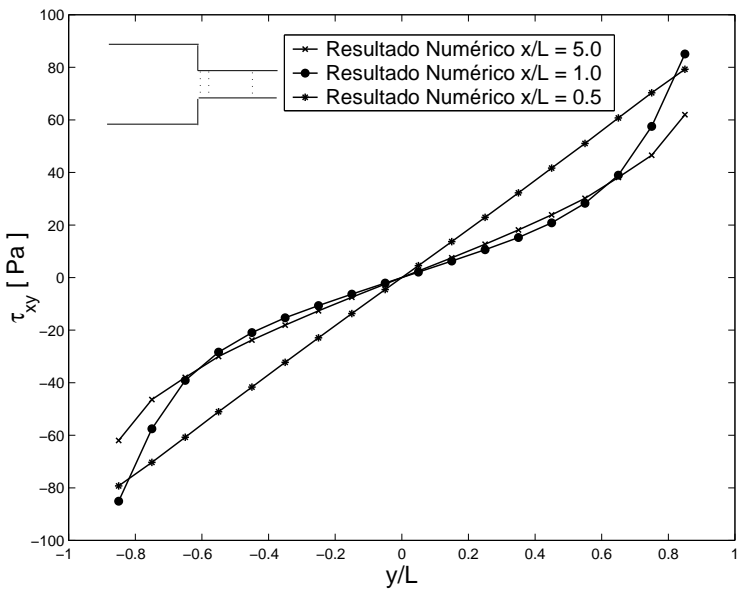

Figura 4.19: Simulação numérica do escoamento em uma contração com os dados de Quinzani et al. [52] $(R e=0,56$ e $D e=2,9)$. a) Valores obtidos experimentalmente para a tensão $\tau^{x y}(y)$ para diversos valores e $x / L$ no canal menor. b) Valores obtidos numericamente nos mesmos pontos.

a)

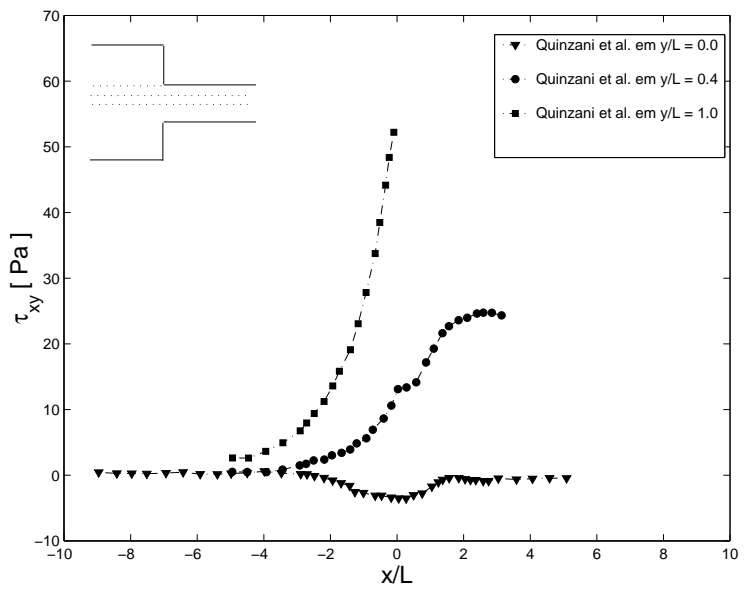

b)

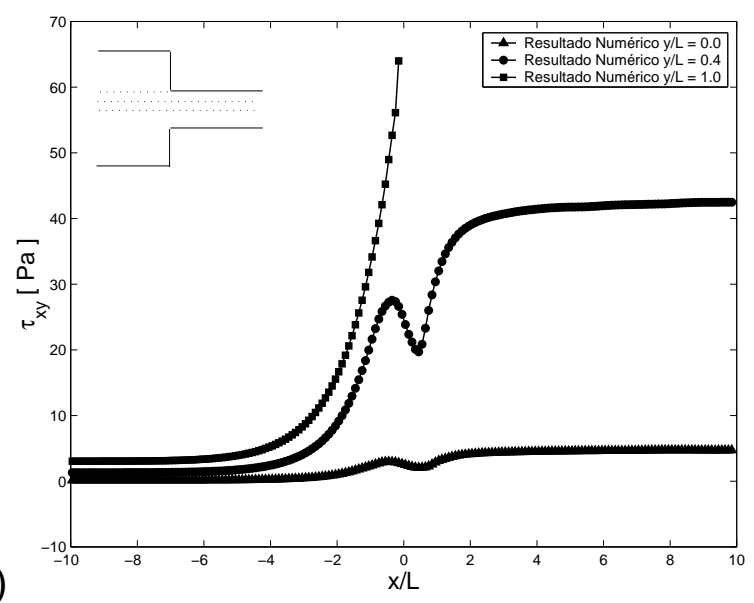

Figura 4.20: Simulação numérica do escoamento em uma contração com os dados de Quinzani et al. [52] $(R e=0,56$ e $D e=2,9)$. a) Valores obtidos experimentalmente para a tensão $\tau^{x y}(x)$ para diversos valores e $y / L$. b) Valores obtidos numericamente nos mesmos pontos.

para a componente de tensão $\tau_{x y}$ em perfis horizontais são ligeiramente melhores neste caso (ver figura 4.24). 
a)

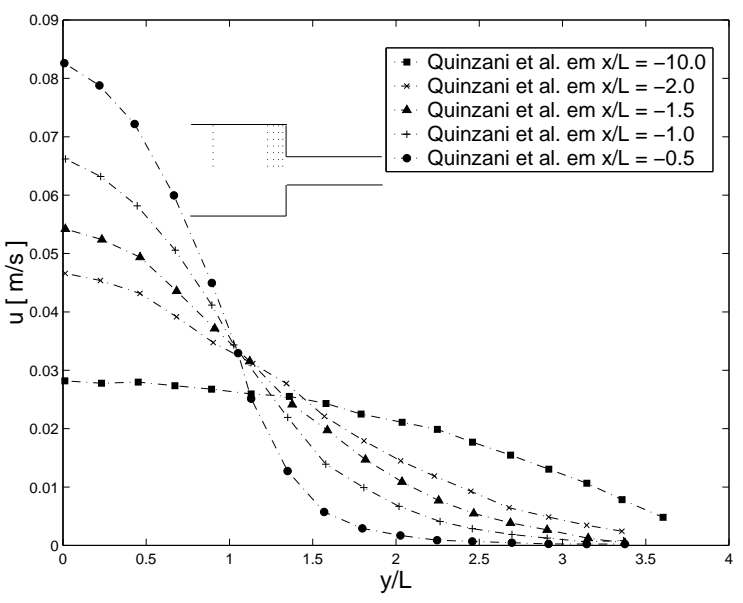

b)

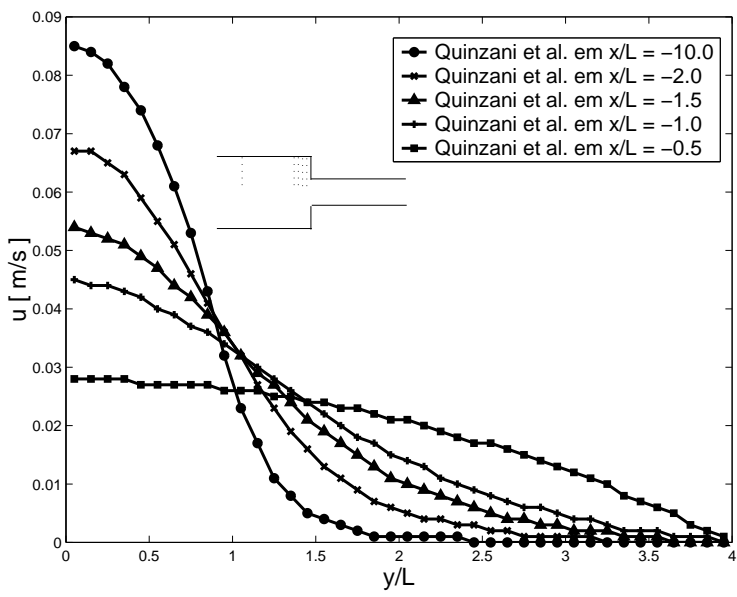

Figura 4.21: Simulação numérica do escoamento em uma contração com os dados de Quinzani et al. [52] $(R e=0,27$ e $D e=1,39)$. a) Valores obtidos experimentalmente para a velocidade $u(y)$ para diversos valores de $x / L$. b) Valores obtidos numericamente nos mesmos pontos.

a)

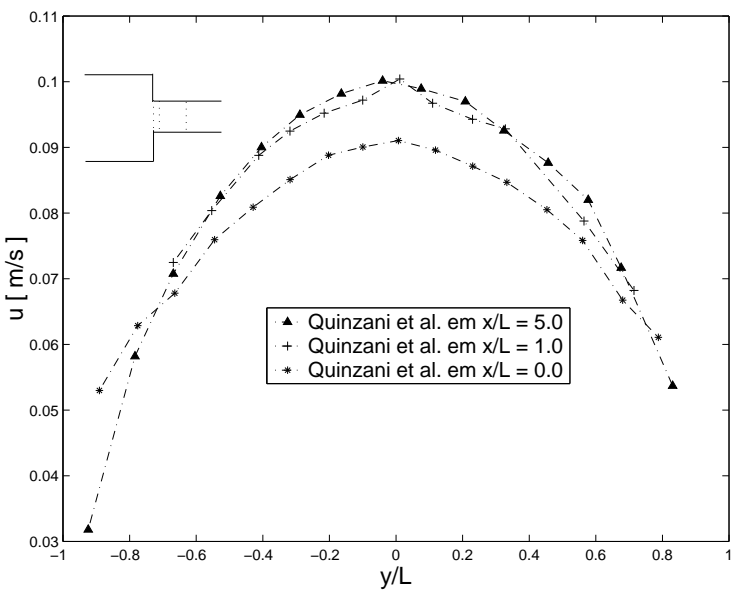

b)

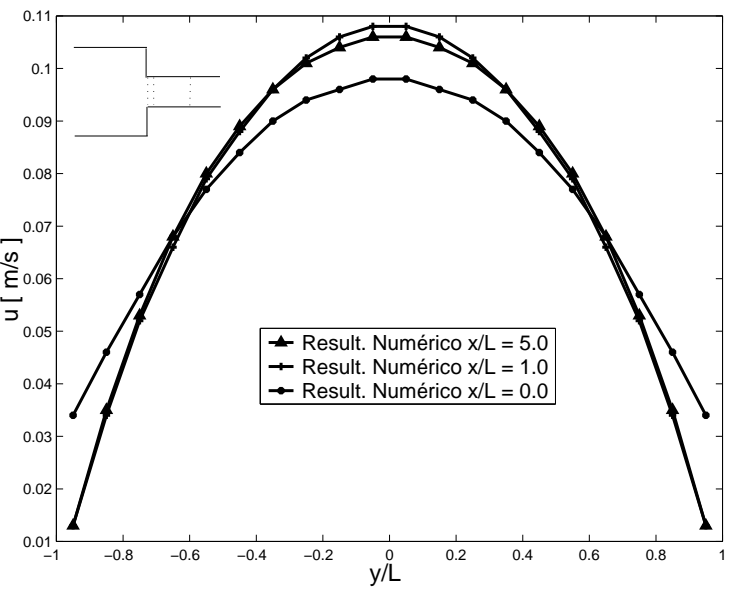

Figura 4.22: Simulação numérica do escoamento em uma contração com os dados de Quinzani et al. [52] $(R e=0,27$ e $D e=1,39)$. a) Valores obtidos experimentalmente para a velocidade $u(y)$ para diversos valores de $x / L$ no canal menor. b) Valores obtidos numericamente nos mesmos pontos.

\subsection{Simulação Numérica do Escoamento em uma Con- tração Planar 4:1}

Nesta seção são apresentados resultados numéricos da simulação do escoamento em uma contração planar 4:1. Nesse problema, o fluido escoa de um canal com largura 8h para 
a)

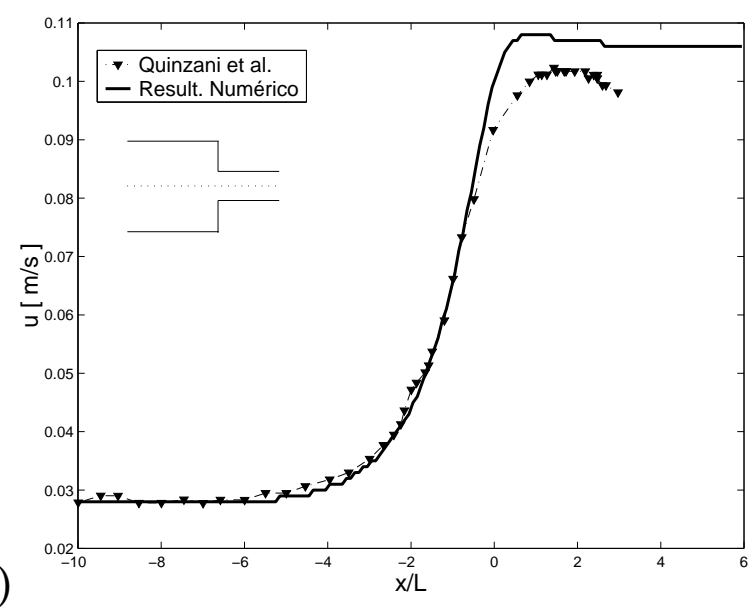

b)

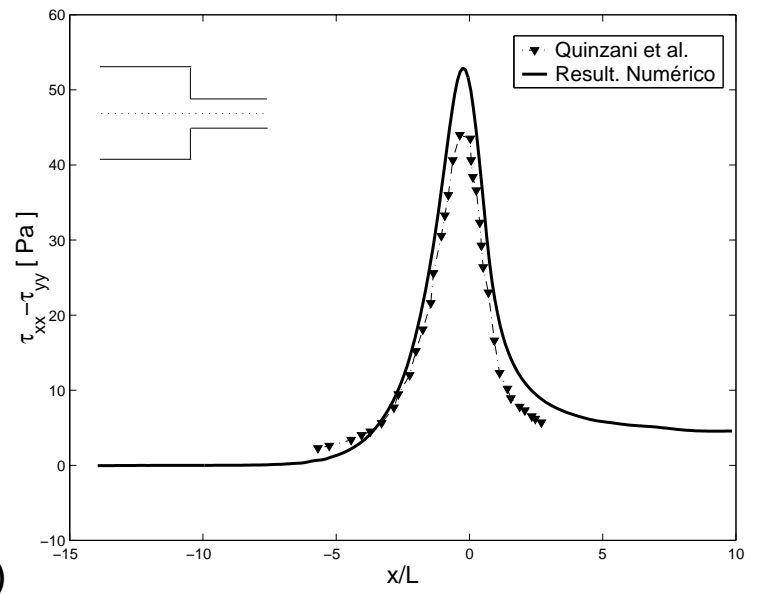

Figura 4.23: Simulação numérica do escoamento em uma contração com os dados de Quinzani et al. [52] $(R e=0,27$ e $D e=1,39)$. a) Valores obtidos experimentalmente para a velocidade $u$ ao longo do eixo de simetria: ( $\mathbf{\nabla})$ Quinzani et al. [52]; (-) FreeFlow2D. b) Valores obtidos numericamente para a primeira diferença de tensões normais $N 1$ nos mesmos pontos: ( $)$ Quinzani et al. [52]; (-) FreeFlow2D.

a)

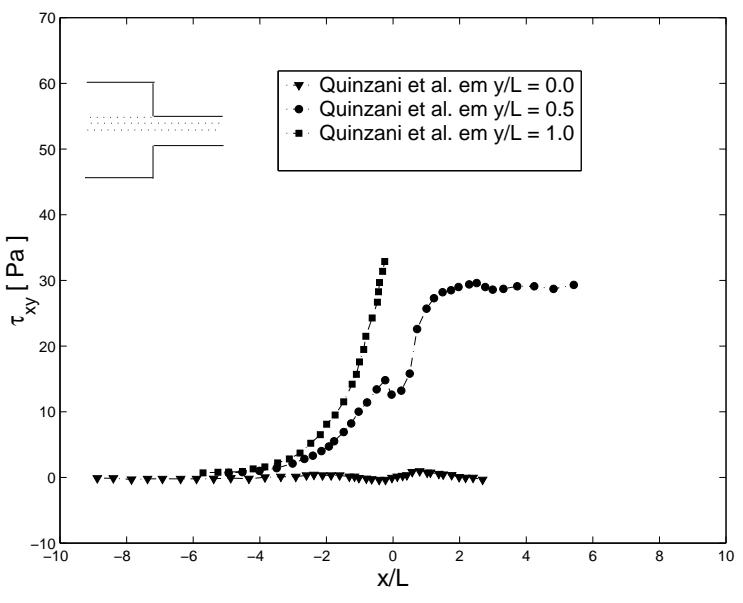

b)

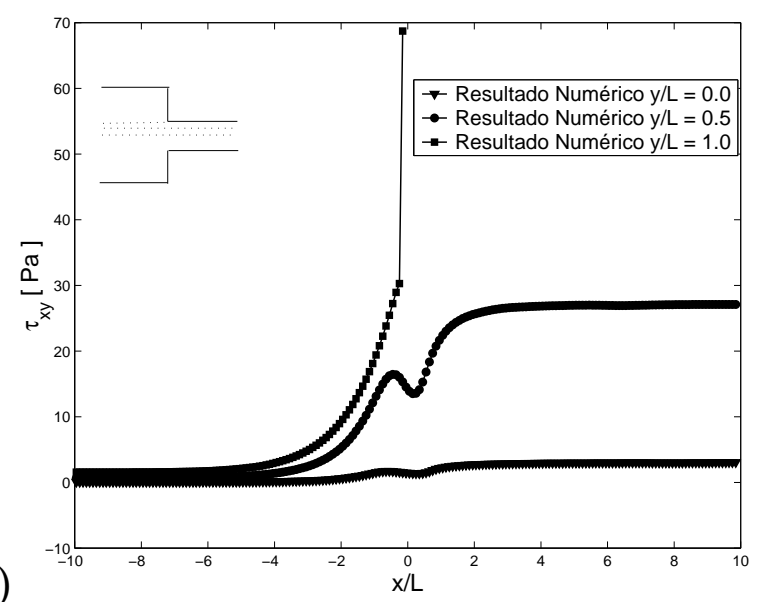

Figura 4.24: Simulação numérica do escoamento em uma contração com os dados de Quinzani et al. [52] ( $R e=0,27$ e $D e=1,39)$. a) Valores obtidos experimentalmente para a tensão $\tau^{x y}(x)$ para diversos valores e $y / L$. b) Valores obtidos numericamente nos mesmos pontos.

outro canal com largura $2 \mathrm{~h}$, de modo que a razão entre as larguras dos canais é 4:1. A geometria do escoamento é mostrada na figura 4.25 .

A simulação numérica da contração planar é um dos instrumentos clássicos usados no desenvolvimento de métodos numéricos para fluidos não-newtonianos. O interesse na simulação deste tipo de escoamento vem do fato de que os fluidos não-newtonianos 


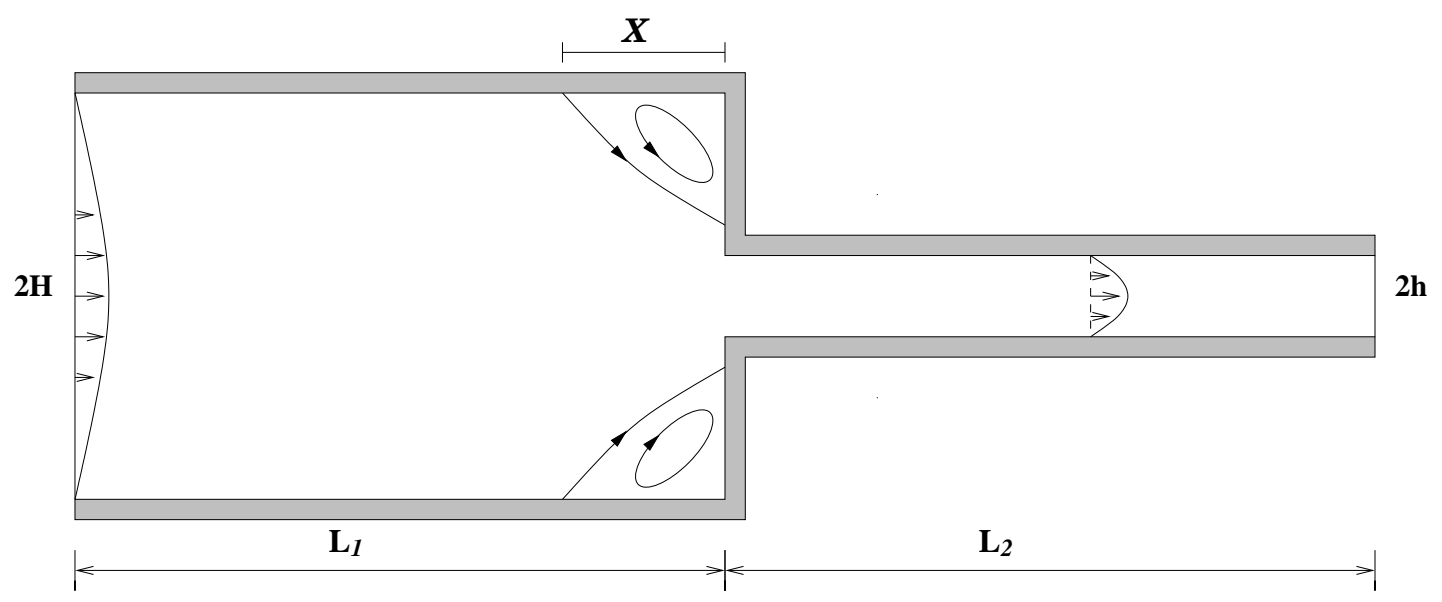

Figura 4.25: Domínio computacional para a simulação do escoamento em uma contração planar 4:1.

apresentam comportamento variado quando sujeitos a esse tipo de geometria. Um ponto particular de interesse é o estudo do comportamento de vórtices nos cantos e próximo às quinas da entrada da contração (conhecidos como lip vortices). O comprimento e a intensidade desses vórtices varia com o número de Weissenberg (ou número de Deborah), com o número de Reynolds, com o tipo de contração e também com a razão de contração, além de propriedades reológicas de cada material como as viscosidades em cisalhamento e em elongação.

Uma questão importante no desenvolvimento dos métodos numéricos é a capacidade de simular esses problemas com números de Weissenberg altos, pois nessas condições os altos níveis de tensão podem causar instabilidades no algoritmo que podem levar a perda da convergência. Diversos são os trabalhos que se dedicaram a descrever o comportamento de fluidos viscoelásticos na contração 4:1, tanto para o problemas planares quanto para problemas axissimétricos. Assim como diversos são os comportamentos obtidos experimentalmente, onde até mesmos fluidos com basicamente as mesmas propriedades viscométricas apresentam padrões de comportamento completamente diferentes, quando sujeitos a escoamentos com a mesma geometria (Boger et al. [9]). Isso mostra um pouco da dificuldade em descrever o comportamento reológico nesses tipos de escoamentos. No que se refere aos trabalhos numéricos, os resultados apresentados variam com o tipo de metodologia utilizada, com a equação constitutiva e também podem variar com o refinamento da malha utilizada. Uma boa revisão da literatura sobre o estudo da contração pode ser encontrada no trabalho de White et al. [70], Walters et al. [66] e também no 
recente livro de Owens e Phillips [46].

Evans e Walters [16] fazem considerações sobre a influência de pequenas mudanças na geometria da entrada do canal de saída do escoamento. É mostrado que o mecanismo de crescimento dos vórtices varia com a razão da contração. São estudados experimentalmente tanto fluidos com viscosidade constante quanto com viscosidade que decai com a taxa de cisalhamento ("shear-thinning fluids"), e é relatado um forte realce nos vórtices para fluidos "shear-thinning" e dificuldades na observação de vórtices para fluidos de Boger ${ }^{1}$. Os mesmos autores mostram em um trabalho subseqüente [17] que o mecanismo de 'lip vortices' pode ser responsável pelo realce nos vórtices para qualquer contração planar, desde que a concentração de polímeros na solução seja escolhida apropriadamente, ou então que o ângulo de contração seja variado.

White e Baird [67] investigaram experimentalmente o comportamento de um poliestireno (PS) e um polietileno de baixa densidade (LDPE) em uma contração planar 4:1 e não observaram o aparecimento de vórtices no fluido PS. É sugerido que o aparecimento de vórtices depende das propriedades extensionais do polímero, em particular do crescimento de tensões extensionais, e portanto das características moleculares do fluido, eliminando assim a categorização de fluidos como aqueles que apresentam ou não vórtices, uma vez que nem todo PS apresenta vórtices. Com isso ainda é sugerido que simulações numéricas para este tipo de contração devam ser feitas com equações constitutivas mais realistas, capazes de prever as propriedades extensionais do fluido em estudo. Essas idéias de dependência com as propriedades extensionais foram verificadas experimentalmente em um trabalho posterior, usando os mesmos materiais [68] e também numericamente [69], onde os autores usam o modelo constitutivo PTT (Phan-Thien-Tanner) para descrever os resultados observados experimentalmente. Os autores procuraram mostrar que o uso de um modelo constitutivo mais realista, capaz de prever melhor as respostas extensionais, poderia ser usado com maior sucesso na descrição do comportamento de vórtices na contração. A importância dessas propriedades extensionais foi mostrada através da variação do parâmetro $\epsilon$ no modelo constitutivo.

Purnode e Crochet [51] usaram o modelo FENE-P para simular resultados obtidos por Evans e Walters ([16],[17]) em uma contração planar. Foram investigadas a influência

\footnotetext{
${ }^{1}$ Fluidos de Boger são fluidos compostos por baixa concentração de polímeros de alto peso molecular dissolvidos em um liquido newtoniano muito viscoso ([26, 27])
} 
da razão de contração, da concentração de polímeros e também do formato das quinas de entrada. Os resultados mostraram uma concordância qualitativa com experimentos inclusive com o aparecimento de 'lip vórtices' para diversos números de Reynolds. Mompean e Deville [40] utilizaram um algoritmo baseado no método MAC juntamente com o método de volumes finitos para a simulação do escoamento em uma contração planar, em duas e três dimensões, para fluidos Oldroyd-B, onde é relatado uma diferença considerável entre os resultados em 2D e 3D. Não há aparecimento de lip vórtices no caso 2D e o comprimento dos vórtices de canto diminui à medida que o número de Weissenberg é aumentado. A simulação em 3D também é o assunto do trabalho de Xue et al. [71], onde o escoamento em uma contração planar 4:1 é simulada usando os modelos de Maxwell para fluidos com viscosidade constante e o modelo PTT para fluidos com viscosidade decrescente com a taxa de cisalhamento. Os resultados indicaram que medidas experimentais podem ser bem reproduzidas se o fluido estiver bem caracterizado por um modelo viscoelástico apropriado. A variação dos resultados com o refinamento da malha também é discutida para o caso 2D de um fluido Oldroyd-B, sendo mostrado que resultados muito diferentes foram obtidos, no que se refere às atividades de vórtice.

Com relação à utilização de modelos integrais, para a simulação de escoamentos em contrações, os trabalhos disponíveis também não são raros. Viriyayuthakorn e Caswell [65] utilizam a simulação numa contração axissimética para apresentar a técnica de calcular a trajetória da partícula no modelo integral de Maxwell. Os resultados para este problema mostraram um aumento no vórtice quando o número de Deborah foi incrementado de um para dois. Lou e Mitsoulis [31] apresentaram um método de elementos finitos para simulação da contração axissimétrica usando o modelo K-BKZ. Um estudo do efeito da viscosidade extensional sobre o comportamento dos vórtices foi apresentado, mostrando mudanças tanto no tamanho quanto no aparecimento ou não de 'lip vórtices'. Esse estudo foi feito através da variação do parâmetro $\beta$ da equação constitutiva (veja eq. 3.11). Em um trabalho posterior Luo [29] apresentou um esquema numérico baseado no método de volumes finitos juntamente com um método ADI para resolver as equações de conservação de quantidade de movimento sobre uma malha deslocada. Os resultados da simulação do escoamento em uma contração 5,75:1 usando o modelo K-BKZ são apresentados e é relatado uma melhoria no método numérico em comparação ao método de elementos finitos usado anteriormente em [31]. 
No artigo de Olley et al. [45] é apresentado um método numérico para obter solução numérica de escoamentos viscoelásticos baseado em equações constitutivas na forma integral. O método é aplicado na simulação do escoamento de um LDPE numa contração 4:1 planar e axissimétrica. A função amortecimento utilizada é a mesma usada por Papanastasiou et al. [47]. Resultados para o caso planar mostraram decrescimento dos vórtices de canto com o aumento da vazão de escoamento imposta; um resultado não esperado para um fluido LDPE. É sugerido que a pouca capacidade da equação constitutiva, com a função amortecimento de PSM, em prever dados em elongação contribuem para esse resultado inesperado. Isso é verificado em um trabalho posterior de Olley [43], onde é apresentada uma modificação da função amortecimento de Papanastasiou et al. [47] de modo a permitir múltiplos parâmetros $\beta$ na equação constitutiva. Esse procedimento permitiu um melhor ajuste de dados experimentais, o que se refletiu numa previsão melhor dos vórtices de canto.

Comparações entre resultados numéricos e experimentais também podem ser encontradas nos trabalhos de Mitsoulis [35, 36, 38, 37, 39], tanto para contrações axissimétricas [36, 38, 37] quanto para planares [35, 38, 39]). É utilizada a função amortecimento modificada por Olley [43] e os resultados obtidos para a contração axissimétrica são considerados bons. Para o caso planar, os resultados em [38] não foram satisfatórios. Os vórtices mostraram um decréscimo no tamanho com o aumento do número de Weissenberg, o que não concordava com resultados experimentais para o mesmo tipo de fluido. A explicação para o ocorrido foi de que os parâmetros nos modelos de Olley deveriam ser modificados a fim de que fosse possível prever melhor os dados experimentais tanto em escoamentos elongacionais uniaxiais quanto planares. Uma correção nesses parâmetros mostrou resultados melhores da contração planar em relação aos observados em laboratório.

Outros trabalhos sobre a simulação na contração planar estão disponíveis na literatura especializada. Uma boa revisão destes resultados pode ser encontrada nos artigos de White et al. [70], Walters et al. [66] e também no livro de Owens e Phillips [46].

Nas seções que se seguem iremos descrever os resultados obtidos com a aplicação do método numérico descrito nos capítulos anteriores na simulação numérica do escoamento em uma contração planar 4:1, usando os modelo constitutivos integrais de Maxwell e K-BKZ. 


\subsubsection{Modelo Maxwell}

Nesta seção apresentamos os resultados numéricos obtidos na simulação do escoamento através de uma contração planar 4:1 utilizando o modelo integral de Maxwell. São apresentados resultados obtidos para os números de Reynolds $R e=0,1$ e $R e=1$ para vários valores do número de Weissenberg. Os dados de entrada utilizados foram:

- Velocidade no injetor: perfil parabólico no injetor com velocidade máxima $V=$ $0,025 \mathrm{~ms}^{-1}$

- Comprimento das cavidades: $L_{1}=16 \mathrm{~cm}$ e $L_{2}=16 \mathrm{~cm}$.

- Altura das cavidades: $2 H=8 \mathrm{~cm}$ e $2 h=2 \mathrm{~cm}$;

- Definição da malha: $\delta x=\delta y=0,1 \mathrm{~cm}($ malha com $(80 \times 320)$-células com um total de 16000 células cheias de fluido);

- Tolerância para a resolução da equação de Poisson para $\psi: E P S=10^{-10}$;

- Força de gravidade nula $g=0$;

- Massa específica do fluido: $\rho=1000 \mathrm{~kg} \mathrm{~m}^{-3}$; Viscosidade do fluido $\eta_{0}=10 \mathrm{~Pa} . \mathrm{s}$;

- Número de subintervalos utilizados $N=50$.

Os parâmetros de escala utilizados na adimensionalização foram: $U=0,1 \mathrm{~ms}^{-1}, L=$ $0,01 \mathrm{~m}$ e $\lambda_{\text {ref }}=\lambda$. Primeiramente, utilizamos $R e=\rho U L / \eta_{0}=0,1$ nas simulações. No modelo de Maxwell, o valor de $\lambda$ e portanto também o valor de $a\left(a=\eta_{0} / \lambda\right)$, foram calculados em função do número de Weissenberg. Nos resultados que seguem, foram utilizados os seguintes números de Weissenberg:

$$
R e=0,1 \begin{cases}W e=0,25 \Longrightarrow \lambda=0,025 \mathrm{~s}, & a=400,000 \mathrm{~Pa} \\ W e=0,50 \Longrightarrow \lambda=0,050 \mathrm{~s}, & a=200,000 \mathrm{~Pa} \\ W e=1,00 \Longrightarrow \lambda=0,100 \mathrm{~s}, & a=100,000 \mathrm{~Pa} \\ W e=1,50 \Longrightarrow \lambda=0,150 \mathrm{~s}, & a=66,666 \mathrm{~Pa} \\ W e=2,00 \Longrightarrow \lambda=0,200 \mathrm{~s}, & a=50,000 \mathrm{~Pa} \\ W e=2,50 \Longrightarrow \lambda=0,250 \mathrm{~s}, & a=40,000 \mathrm{~Pa} \\ W e=3,00 \Longrightarrow \lambda=0,300 \mathrm{~s}, & a=33,333 \mathrm{~Pa} \\ W e=3,50 \Longrightarrow \lambda=0,350 \mathrm{~s}, & a=28,571 \mathrm{~Pa} \\ W e=4,00 \Longrightarrow \lambda=0,400 \mathrm{~s}, & a=25,000 \mathrm{~Pa}\end{cases}
$$




$$
R e=1,0 \begin{cases}W e=0,25 \Longrightarrow \lambda=0,0025 \mathrm{~s}, & a=4000,000 \mathrm{~Pa} \\ W e=0,50 \Longrightarrow \lambda=0,0050 \mathrm{~s}, & a=2000,000 \mathrm{~Pa} \\ W e=1,00 \Longrightarrow \lambda=0,0100 \mathrm{~s}, & a=1000,000 \mathrm{~Pa} \\ W e=1,50 \Longrightarrow \lambda=0,0150 \mathrm{~s}, & a=666,666 \mathrm{~Pa} \\ W e=2,00 \Longrightarrow \lambda=0,0200 \mathrm{~s}, & a=500,000 \mathrm{~Pa} \\ W e=2,50 \Longrightarrow \lambda=0,0250 \mathrm{~s}, & a=400,000 \mathrm{~Pa} \\ W e=3,00 \Longrightarrow \lambda=0,0300 \mathrm{~s}, & a=333,333 \mathrm{~Pa} \\ W e=3,50 \Longrightarrow \lambda=0,0350 \mathrm{~s}, & a=285,710 \mathrm{~Pa} \\ W e=4,00 \Longrightarrow \lambda=0,0400 \mathrm{~s}, & a=250,000 \mathrm{~Pa}\end{cases}
$$

Utilizando os dados descritos acima, o código FreeFlow-2D simulou o problema da contração planar 4:1 para os números de Weissenberg $W e=0$ (newtoniano), 0, 25, 0, 5, $\cdots, 4$, até que o estado estacionário fosse atingido e calculamos o tamanho do vórtice de canto obtido para cada $W e$. Um total de 10 simulações foram efetuadas para cada Re. As figuras 4.26 e 4.27 mostram as linhas de corrente obtidas nas simulações para cada $W e$. A tabela 4.2 mostra o tamanho dos vórtices, dado por $\mathrm{L}_{\mathrm{vortex}}=X / h$ (ver figura 4.25), obtidos para cada valor de $W$ e e a figura 4.28 ilustra a variação do tamanho do vórtice com o crescimento do número de Weissenberg. Podemos observar nas figuras 4.26 e 4.27 que à medida que o número de Weissenberg aumenta, o tamanho do vórtice de canto diminui devido aos efeitos viscoelásticos. Além disso, para $W e=4$, os resultados mostram o aparecimento de um pequeno vórtice perto do canal de entrada (conhecido como "lip vortex") para ambos os valores de Re. Esse problema tem sido simulado por vários autores utilizando modelos diferenciais tais como Oldroyd-B, Maxwell e os resultados mostraram que, de fato, o tamanho do vórtice de canto diminui com o aumento da viscoelasticidade (representada pelo $W e$ ). O aparecimento do "lip vortex" tem sido reportado por muitos autores. Por exemplo, Grossi [19] simulou o problema da contração 4:1 utilizando o modelo Oldroyd-B e obteve um "lip vortex" para o escoamento a baixo número de Reynolds $R e=0,01$ e $W e=2,5$ e $W e=3$. Porém, os resultados apresentados por Grossi [19] para $R e=1$ não mostram o aparecimento do "lip vortex". Alves et al. [1] também estudaram o problema da contração planar 4:1 utilizando o modelo Oldroyd-B. Para escoamentos lentos $(R e=0)$, utilizando o modelo Oldroyd-B, os resultados obtidos por Alves et al. [1] mostram o aparecimento de pequenos "lip vortex" para valores do número de Deborah $D e=0,5,1,1,5$. Phillips e Williams [50] utilizaram um método semi-lagrangiano e 
analisaram o problema da contração planar 4:1. Esses autores apresentaram resultados para escoamentos com $R e=0$ e $R e=1$ e o maior número de Weissenberg alcançado foi $W e=2,5$. Os resultados numéricos para escoamentos lentos, mostraram um pequeno "lip vortex" para números de Weissenberg $W e=2$ e $W e=2,5$. No entanto, os resultados provenientes das simulações com escoamentos inerciais $R e=1$ não mostraram o aparecimento do "lip vortex".

Em resumo, podemos dizer que os resultados obtidos nesse trabalho para $R e=0,1$ concordam qualitativamente com os obtidos por outros autores. Porém, os resultados obtidos com $R e=1$ e $W e=4$ mostram o aparecimento de um "lip vortex". Para confirmar esse resultado, esse problema foi simulado novamente utilizando uma malha mais fina com $(160 \times 640)$-células e o resultado obtido foi semelhante àquele obtido na malha com $(80 \times 320)$-células (ver figura 4.29). Esse fato não tem sido reportado na literatura e acreditamos que necessita de uma maior investigação.

Portanto, os resultados obtidos nessas simulações demonstram que o método numérico desenvolvido nesse trabalho é capaz de simular escoamentos viscoelásticos governados pela equação constitutiva integral de Maxwell.

Tabela 4.2: Variação do comprimento dos vórtices com o número de Weissenberg para o modelo de Maxwell

\begin{tabular}{|c|c|c|c|c|c|c|c|c|c|c|}
\hline \hline $\mathrm{We}$ & 0,0 & 0,25 & 0,5 & 1,0 & 1,5 & 2,0 & 2,5 & 3,0 & 3,5 & 4,0 \\
\hline \hline $\operatorname{Re}=0,1$ & 1,447 & 1,417 & 1,376 & 1,304 & 1,246 & 1,202 & 1,165 & 1,133 & 1,111 & 1,095 \\
\hline $\operatorname{Re}=1,0$ & 1,271 & 1,227 & 1,176 & 1,089 & 1,016 & 0,959 & 0,909 & 0,856 & 0,820 & 0,768 \\
\hline \hline
\end{tabular}

\subsubsection{Modelo K-BKZ}

Nesta seção apresentamos os resultados obtidos na simulação do escoamento em uma contração planar 4:1 usando o modelo constitutivo K-BKZ com a função memória definida com múltiplos tempos de relaxação, ou seja,

$$
\boldsymbol{\tau}(t)=\int_{-\infty}^{t} \sum_{m=1}^{4} \frac{a_{m}}{\lambda_{m} W e} e^{-\frac{t-t^{\prime}}{\lambda_{m} W e}} \frac{\alpha}{\alpha-3+\beta I_{1}+(1-\beta) I_{2}} \mathbf{B}_{t^{\prime}}(t) d t^{\prime}
$$


a)

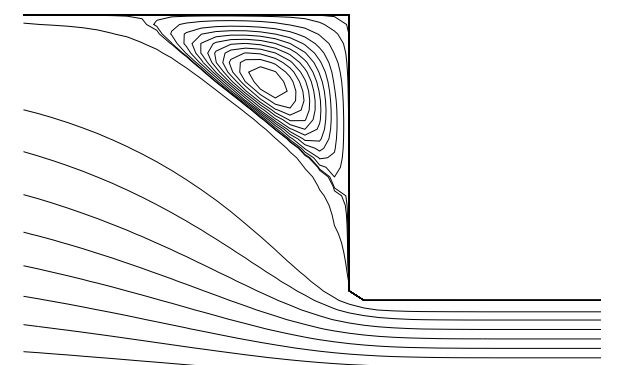

c)

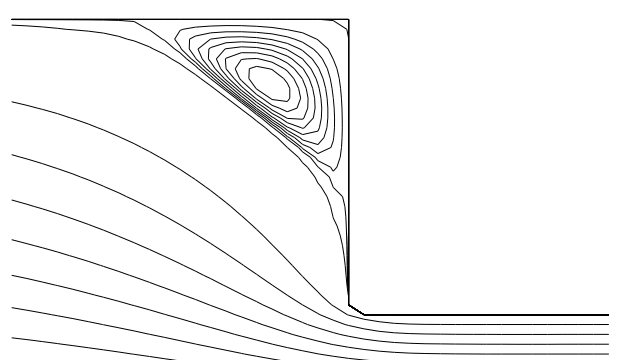

e)

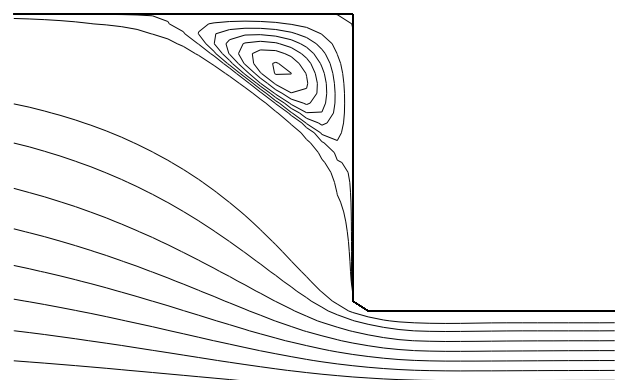

g)
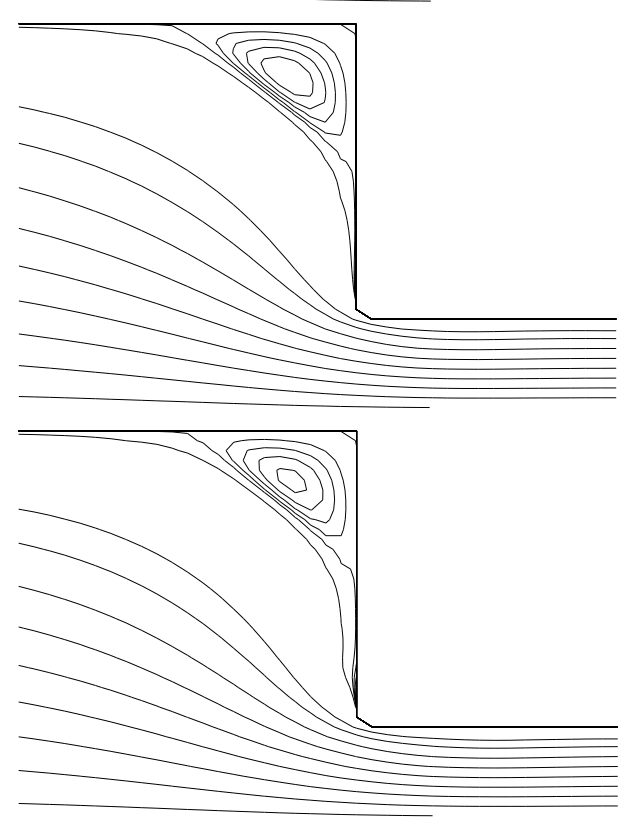

b)

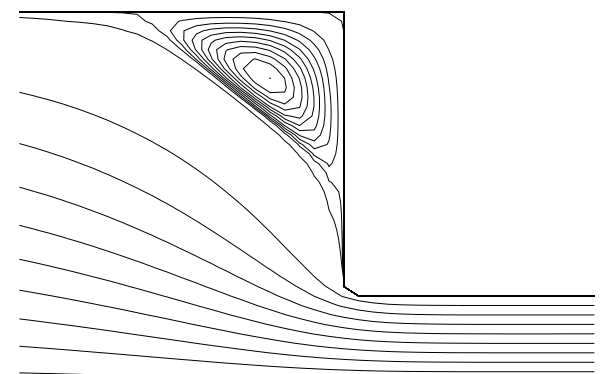

d)

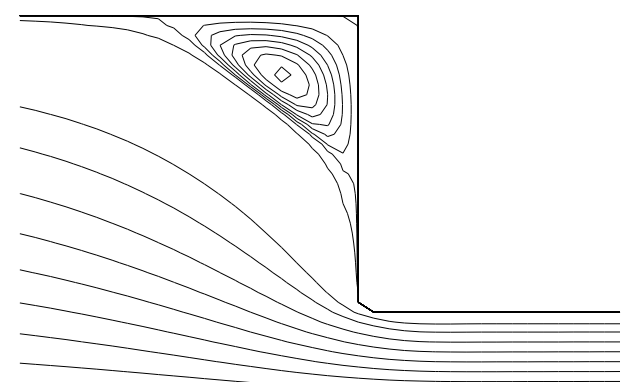

f)

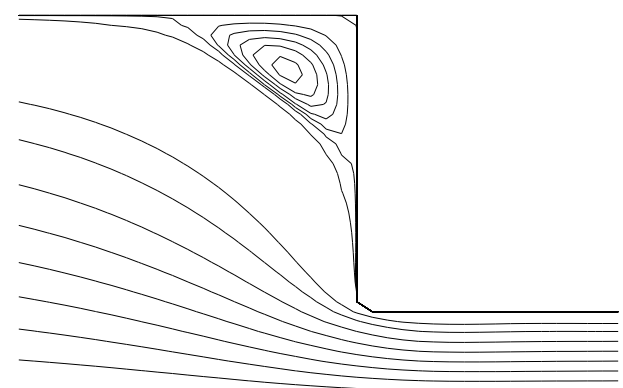

h)

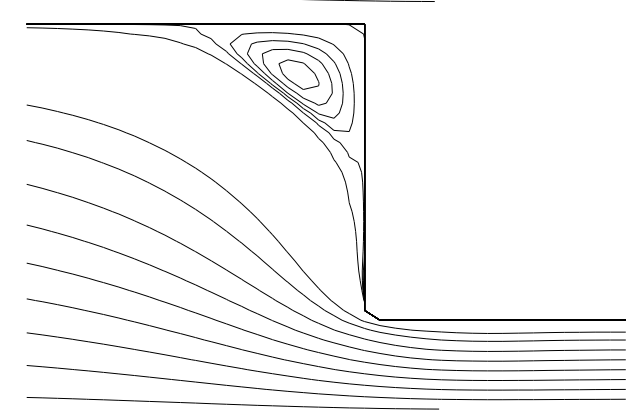

j)

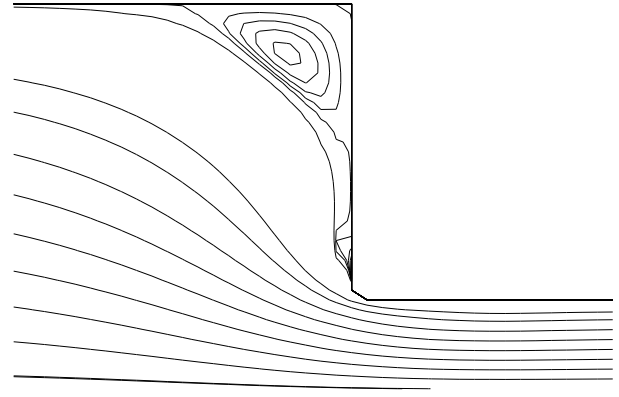

Figura 4.26: Simulação do escoamento em uma contração planar 4:1 pelo modelo de Maxwell. Resultados obtidos com $R e=0,1$. a) $\mathrm{We}=0,0, \mathbf{b}) \mathrm{We}=0,25$, c) $\mathrm{We}=0,50$, d) $\mathrm{We}=1,0, \mathbf{e}) \mathrm{We}=1,5, \mathbf{f}) \mathrm{We}=2,0, \mathbf{g}) \mathrm{We}=2,5, \mathbf{h}) \mathrm{We}=3,0, \mathbf{i}) \mathrm{We}=3,5, \mathbf{j}) \mathrm{We}$ $=4,0$. 
a)
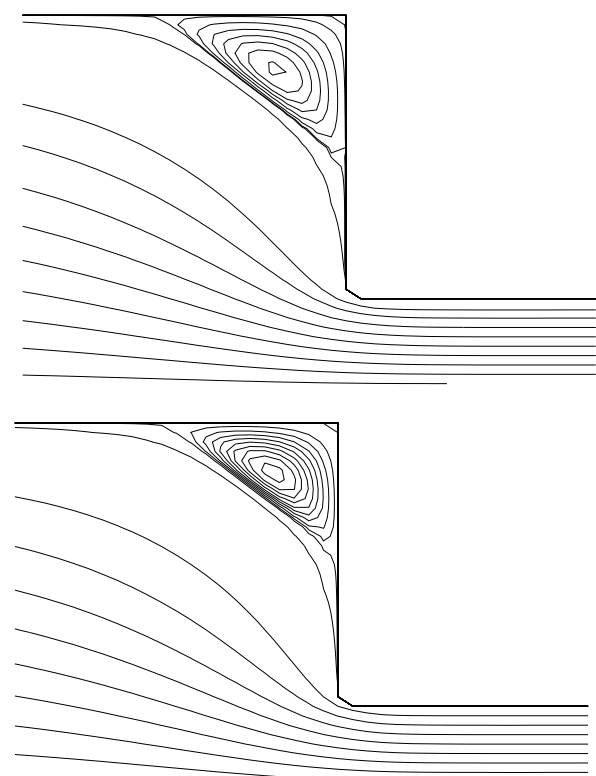

c)

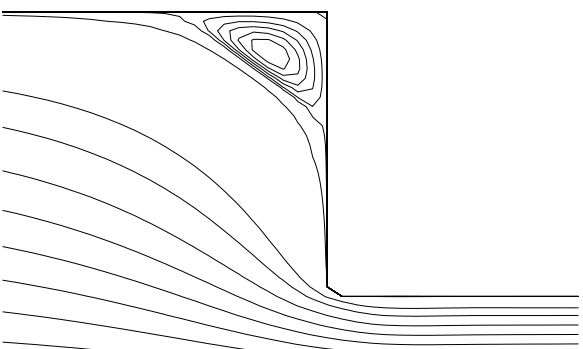

e)

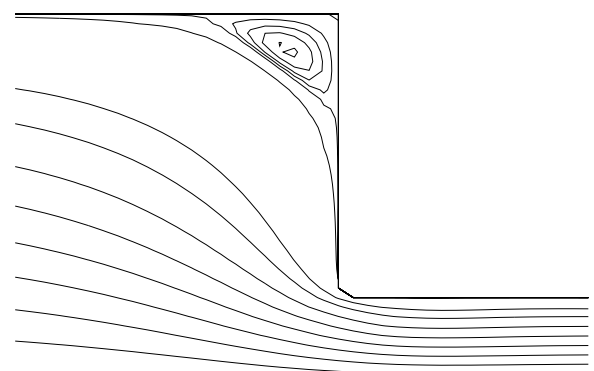

g)

i)

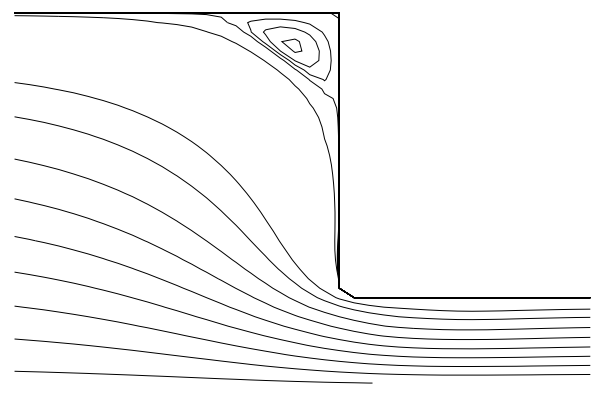

b)

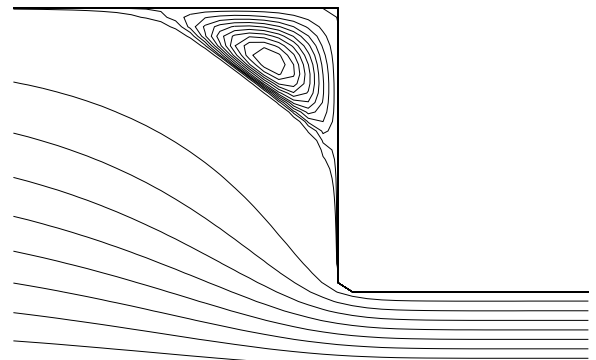

d)

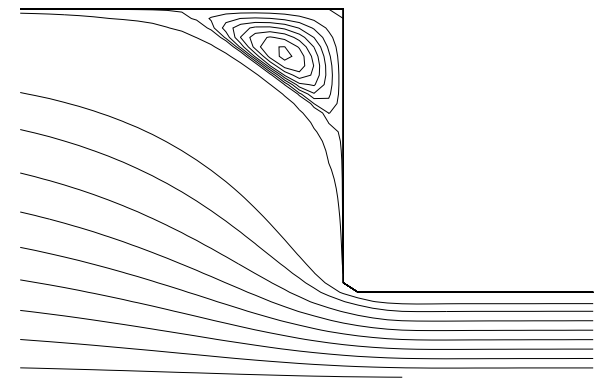

f)

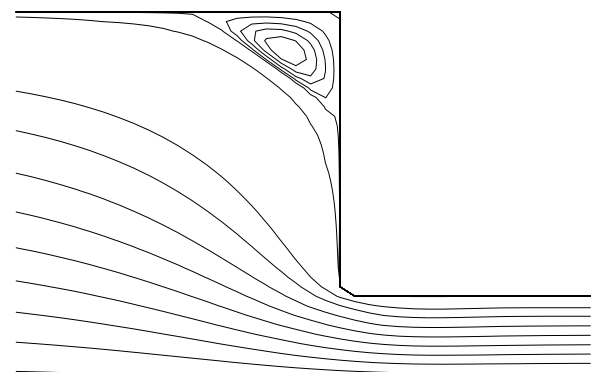

h)

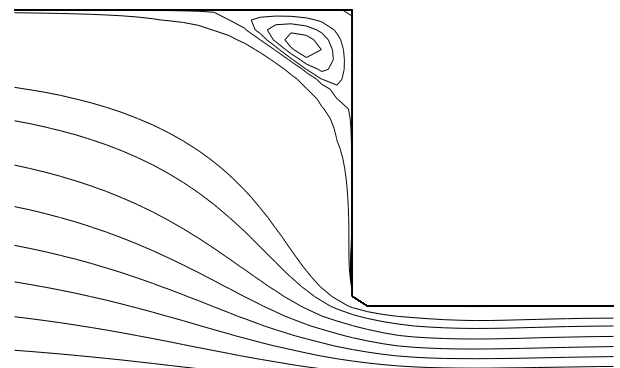

j)

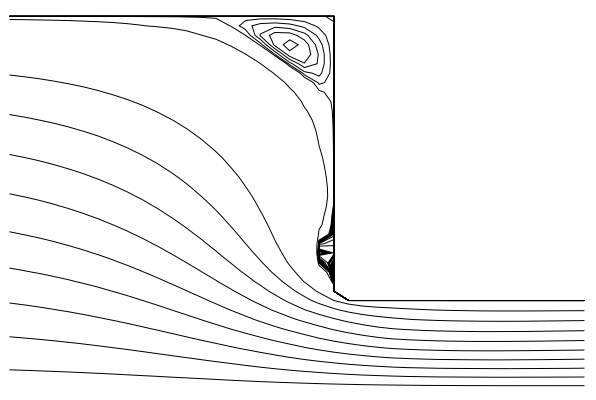

Figura 4.27: Simulação do escoamento em uma contração planar 4:1 pelo modelo de Maxwell. Resultados obtidos com $R e=1,0$. a) $\mathrm{We}=0,0, \mathbf{b}) \mathrm{We}=0,25, \mathbf{c}) \mathrm{We}=0,50$, d) $\mathrm{We}=1,0, \mathbf{e}) \mathrm{We}=1,5, \mathbf{f}) \mathrm{We}=2,0, \mathbf{g}) \mathrm{We}=2,5, \mathbf{h}) \mathrm{We}=3,0, \mathbf{i}) \mathrm{We}=3,5, \mathbf{j}) \mathrm{We}$ $=4,0$ 


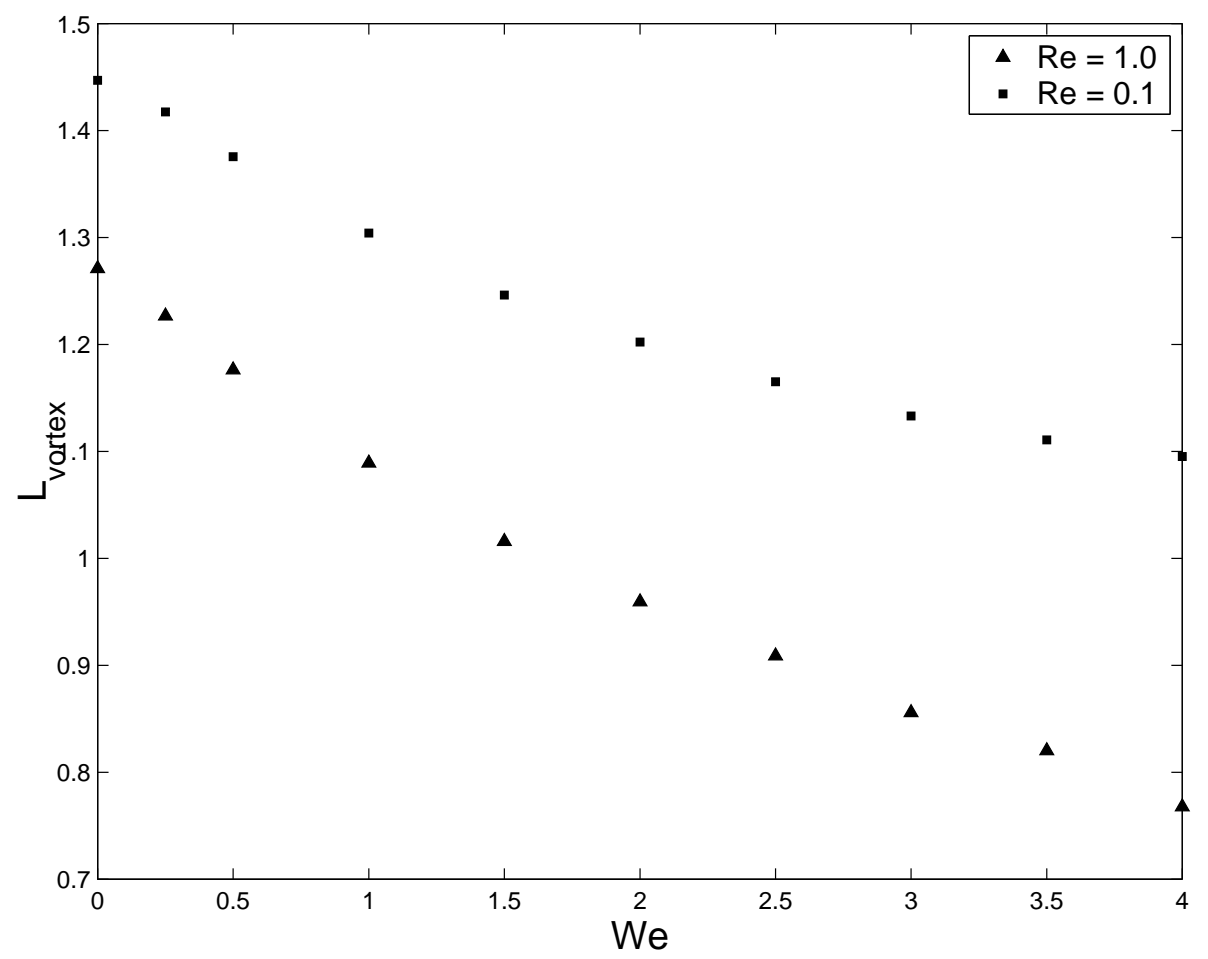

Figura 4.28: Variação do comprimento do vórtice $\left(\mathrm{L}_{\text {vortex }}=X / h\right.$ ver figura 4.25) em função do número de Weissenberg: Modelo de Maxwell

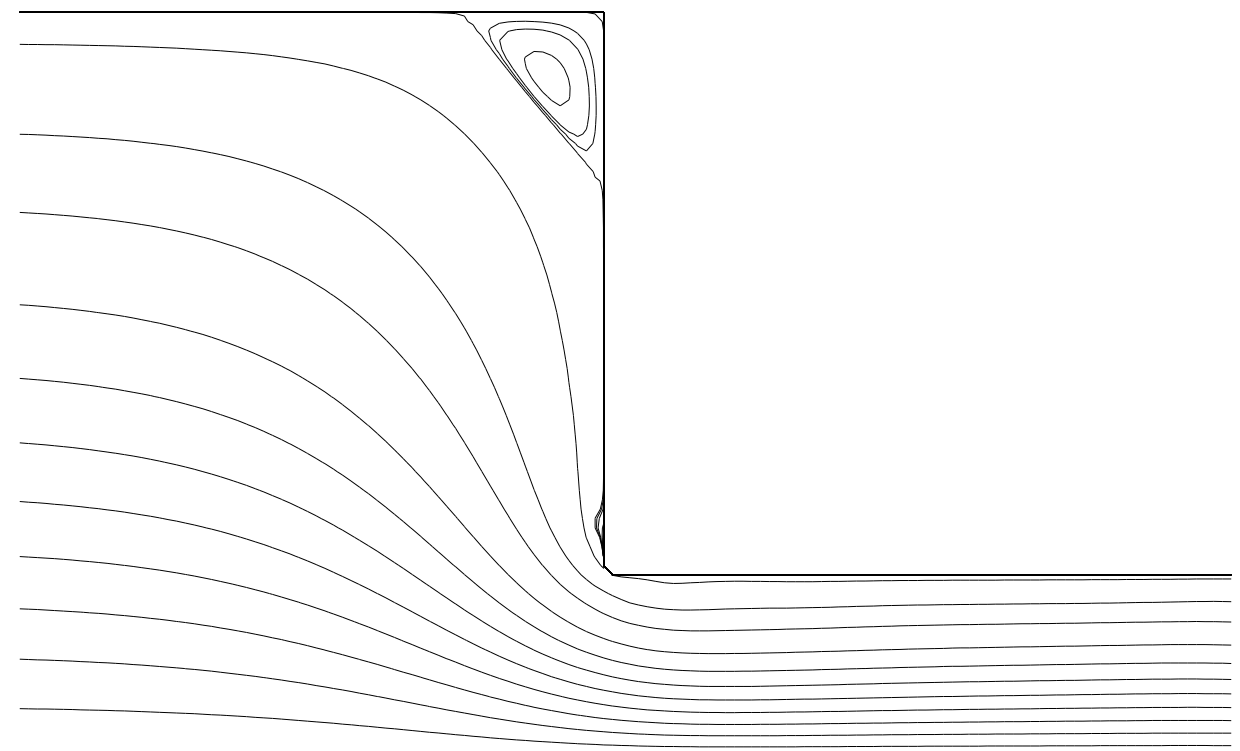

Figura 4.29: Simulação do escoamento em uma contração planar 4:1 usando o modelo de Maxwell: $R e=1$ e $W e=4$. Malha utilizada $(160 \times 640)$-células. 
Os parâmetros utilizados para a definição do fluido foram os mesmos utilizados no artigo de Mitsoulis [37]. Nesse artigo, Mitsoulis [37] apresenta resultados da simulação do escoamento em uma contração axissimétrica 24:1 usando os dados referentes a um fluido S1 (uma solução 2,5 wt\% de poliisobutileno em um solvente de polibuteno), os quais são mostrados na tabela 4.3. Os parâmetros de escala utilizados foram $U=\langle u>$ onde $\langle u\rangle$ representa a velocidade média no canal menor, $L=h$ (ver figura 4.25) e $\lambda_{\text {ref }}=\lambda_{0}$, onde $\lambda_{0}$ representa o tempo de relaxação obtido para o modelo Maxwell. Logo, o número de Weissenberg é dado por

$$
W e=\lambda_{0} \frac{<u>}{h}
$$

onde $\lambda_{0}=2,3 \mathrm{~s}$.

Tabela 4.3: Parâmetros da equação K-BKZ para um fluido S1 (Mitsoulis [37]). $\quad \alpha=25$, $\beta=0,0011, \rho=0,886 \mathrm{~g} \cdot \mathrm{cm}^{3}, \eta_{0}=13,68 \mathrm{~Pa} \mathrm{~s}, \lambda_{0}=2,3 \mathrm{~s}$

\begin{tabular}{ccc}
\hline \hline$k$ & $\lambda_{k}(\mathrm{~s})$ & $a_{k}(\mathrm{~Pa})$ \\
\hline 1 & 0,0078 & 129,49 \\
2 & 0,116 & 27,93 \\
3 & 0,705 & 7,177 \\
4 & 6,48 & 0,65 \\
\hline \hline
\end{tabular}

Para simular esse problema, utilizamos o mesmo domínio computacional utilizado na simulação do escoamento em uma contração 4:1 pelo modelo de Maxwell (ver figura 4.25) e os seguintes dados de entrada:

- Comprimento das cavidades: $L_{1}=160 \mathrm{~cm}$ e $L_{2}=160 \mathrm{~cm}$.

- Altura das cavidades: $2 H=80 \mathrm{~cm}$ e $2 h=20 \mathrm{~cm}$;

- Definição da malha: $\delta x=\delta y=1 \mathrm{~cm}$ (malha com $(80 \times 320)$-células com um total de 16000 células cheias de fluido);

- Tolerância para a resolução da equação de Poisson para $\psi: E P S=10^{-10}$;

- Força de gravidade nula $g=0$;

- Número de subintervalos utilizados $N=50$. 
A escala de comprimento foi $L=10 \mathrm{~cm}$ e a escala de velocidade foi variada para obter o valor do número de Weissenberg desejado. Os valores utilizados foram:

$$
\begin{aligned}
& W e=1 \Longrightarrow U=0,0435 \mathrm{~ms}^{-1} \Longrightarrow R e=0,280 ; \\
& W e=2 \Longrightarrow U=0,0870 \mathrm{~ms}^{-1} \Longrightarrow R e=0,560 ; \\
& W e=3 \Longrightarrow U=0,1305 \mathrm{~ms}^{-1} \Longrightarrow R e=0,845 ; \\
& W e=4 \Longrightarrow U=0,1740 \mathrm{~ms}^{-1} \Longrightarrow R e=1,130 ; \\
& W e=5 \Longrightarrow U=0,2175 \mathrm{~ms}^{-1} \Longrightarrow R e=1,410 ; \\
& W e=6 \Longrightarrow U=0,2609 \mathrm{~ms}^{-1} \Longrightarrow R e=1,689 .
\end{aligned}
$$

O código FreeFlow-2D simulou o problema da contração 4:1 utilizando os dados descritos acima. Devido às baixas velocidades utilizadas, as simulações referentes aos números de Weissenberg $W e=1$ e $W e=2$ foram realizadas até $t=46$ s e as simulações para $W e=3, W e=4, W e=5$ e $W e=6$ foram executadas até $t=20 \mathrm{~s}$. Os resultados obtidos nessas simulações estão mostrados na figura 4.30. Podemos observar na figura 4.30 que o maior vórtice de canto obtido corresponde aos dados $W e=1$ e $R e=0,28$. Além disso, pode-se observar ainda que à medida que o número de Weissenberg aumenta, o tamanho do vórtice de canto diminui, como mostra a tabela 4.4 .

Tabela 4.4: Variação do comprimento dos vórtices obtidos com o modelo KBKZ para diferentes valores de $W e$ e $R e$

\begin{tabular}{|c|c|c|c|c|c|c|}
\hline \hline$(W e, R e)$ & $(1,0,0,280)$ & $(2,0,0,560)$ & $(3,0,0,845)$ & $(4,0,0,130)$ & $(5,0,1,410)$ & $(6,0,1,689)$ \\
\hline \hline $\mathrm{L}_{\text {vortex }}$ & 1,351 & 1,095 & 1,020 & 0,920 & 0,867 & 0,702 \\
\hline \hline
\end{tabular}




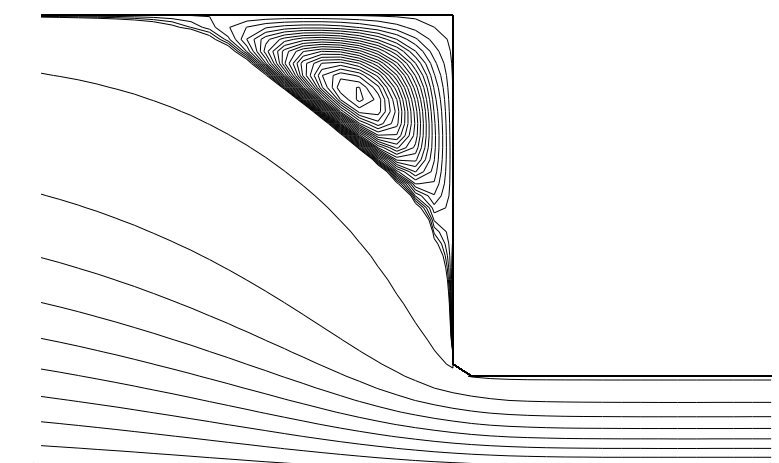

a)

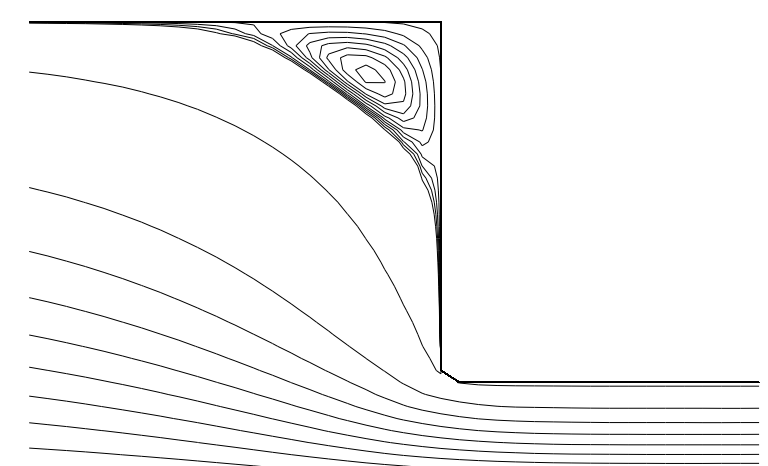

c)

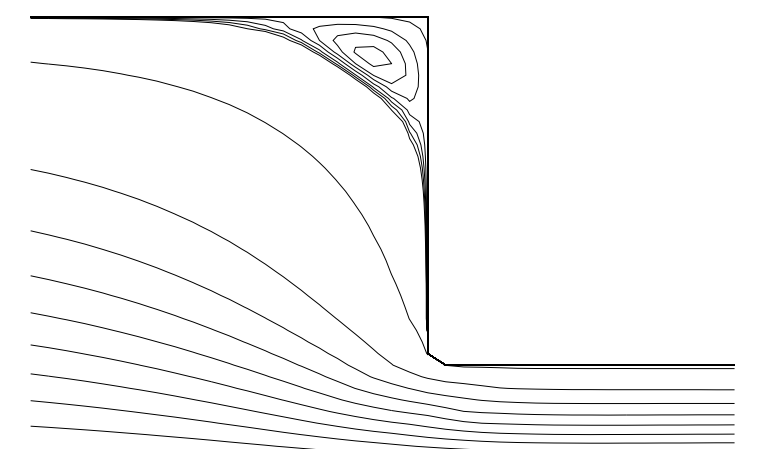

e)

Figura 4.30: Simulação numérica do escoamento em uma contração 4:1 utilizando o modelo K-BKZ para vários números de Weissenberg e vários números de Reynolds: a) $W e=1, R e=0,280 ; \mathbf{b}) W e=2, R e=0,560 ; \mathbf{c}) W e=3, R e=0,845$; $\mathbf{d})$ $W e=4, R e=1,130 ; \mathbf{e}) W e=5, R e=1,410 ; \mathbf{f}) W e=6, R e=1,690$.

\subsection{Simulação Numérica de Escoamentos com Su- perfícies Livres}

Para demonstrar que o método numérico desenvolvido nesta tese pode simular escoamentos viscoelásticos com superfícies livres governados pelos modelos integrais de Maxwell e 
K-BKZ, vamos utilizar o código FreeFlow-2D para simular o problema do jato oscilante ("jet buckling").

\subsubsection{Simulação numérica do jato oscilante}

Quando um jato newtoniano incide sobre uma superfície plana, se o número de Reynolds empregado for menor que um valor crítico, pode ocorrer o fenômeno conhecido como jato oscilante (ver figura 4.31a). Esse problema tem atraído um grande número de pesquisadores e tem sido estudado experimentalmente e numericamente (por exemplo, Cruickshank e Munson [14], Tomé et al. [62] e Tomé e McKee [63]) . Cruickshank e Munson [14] e Cruickshank [13] apresentaram resultados teóricos e numéricos para jatos newtonianos e obtiveram condições necessárias para a ocorrência do efeito do jato oscilante. Essas condições apresentam uma relação entre o número de Reynolds e a razão $H / L$ ( $H$ é a altura entre o injetor e a superfície plana e $L$ é a largura do jato, ver figura 4.31b). Particularmente, os resultados de Cruickshank [13] mostraram que, em um jato bidimensional que sofre oscilação, as seguintes condições são satisfeitas:

$$
R e<0,5 \quad \text { e } \quad H / L>3 \pi .
$$

a)

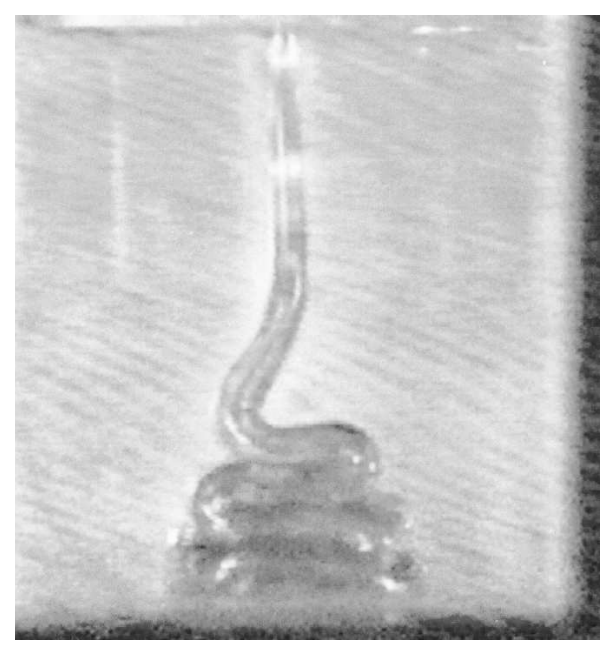

b)

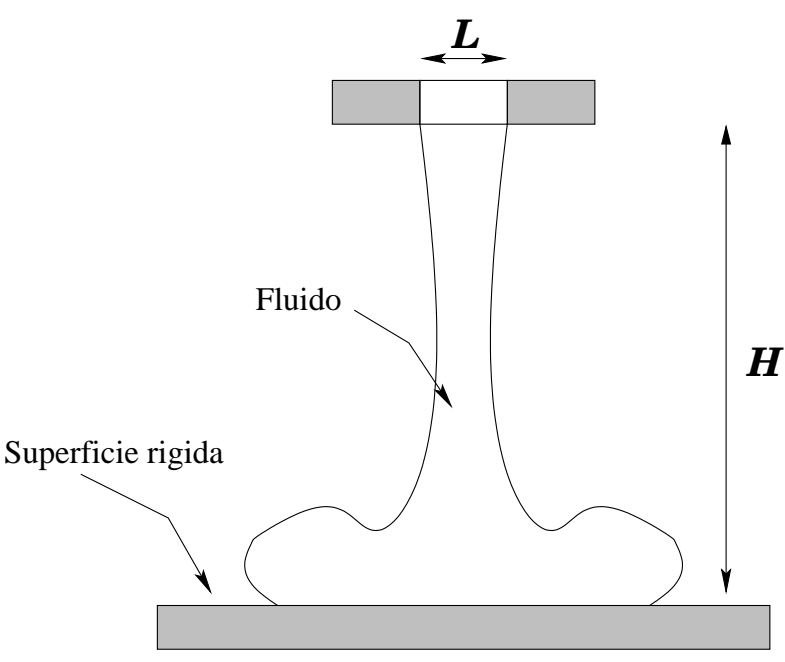

Figura 4.31: Simulação do jato oscilante: a) Resultado experimental do jato oscilante $R e=0,15$ (fluido newtoniano) b) Domínio computacional.

Para ilustrar o fenômeno do jato oscilante em um fluido de Maxwell, apresentamos a seguir duas simulações que mostram os comportamentos de um jato newtoniano e um jato governado pelo modelo Maxwell. Os dados utilizados nessas simulações foram: 
- Domínio computacional: $5,6 \mathrm{~cm} \times 10,6 \mathrm{~cm}$

- Malha utilizada: $\delta x=\delta y=1 \mathrm{~mm}(56 \times 106)$-células

- Aceleração gravitacional: $g=9,81 \mathrm{~ms}^{-2}$

- Altura do injetor à superfície rígida: $H=10 \mathrm{~cm}$

- Largura do injetor: $L=6 \mathrm{~mm}$

- Velocidade do jato no injetor: $U=0,6 \mathrm{~ms}^{-1}$

- Precisão da equação de Poisson: $E P S=10^{-7}$

- Fator para o cálculo do tamanho do passo no tempo: $F A C T=0,1$

- Definição do fluido:

- Viscosidade a baixas taxas de cisalhamento: $\eta_{0}=6 \mathrm{~Pa} . \mathrm{s}$

- Massa específica do fluido: $\rho=1000 \mathrm{kgm}^{-3}$

- Modelo de Maxwell: $a=2000 \mathrm{~Pa}$ e tempo de relaxação $\lambda=0,003 \mathrm{~s}$

As variáveis de escala foram $U, L, \eta_{0}, \rho$ e $\lambda$ de modo que temos:

$$
R e=\rho U L / \eta_{0}=0,6 \quad \text { e } \quad W e=\lambda U / L=0,3
$$

Logo, temos Re $>0,5$ e $H / L=16,7<10$ e, portanto, as condições de Cruickshank não estão satisfeitas e o jato newtoniano não deverá apresentar o efeito de jato oscilante. Com relação ao jato modelado pela equação de Maxwell não podemos concluir que o mesmo não apresentará o efeito do jato oscilante, visto que a análise de Cruickshank é aplicada somente a jatos newtonianos. O código FreeFlow-2D utilizou os dados acima e simulou o jato newtoniano e o jato viscoelástico incidindo sobre uma superfície rígida. A figura 4.32 mostra a simulação do jato newtoniano e o jato não-newtoniano em vários tempos e podemos observar que, como previsto, o jato newtoniano ao atingir a superfície rígida escoa lateralmente, sem apresentar o fenômeno do jato oscilante. Por outro lado, o jato não-newtoniano ao atingir a superfície rígida não consegue escoar lateralmente e o acúmulo de fluido faz com que o mesmo apresente o efeito do jato oscilante. Acreditamos que isso ocorreu devido ao crescimento da viscosidade extensional no momento que o jato não-newtoniano atingiu a superfície rígida. 

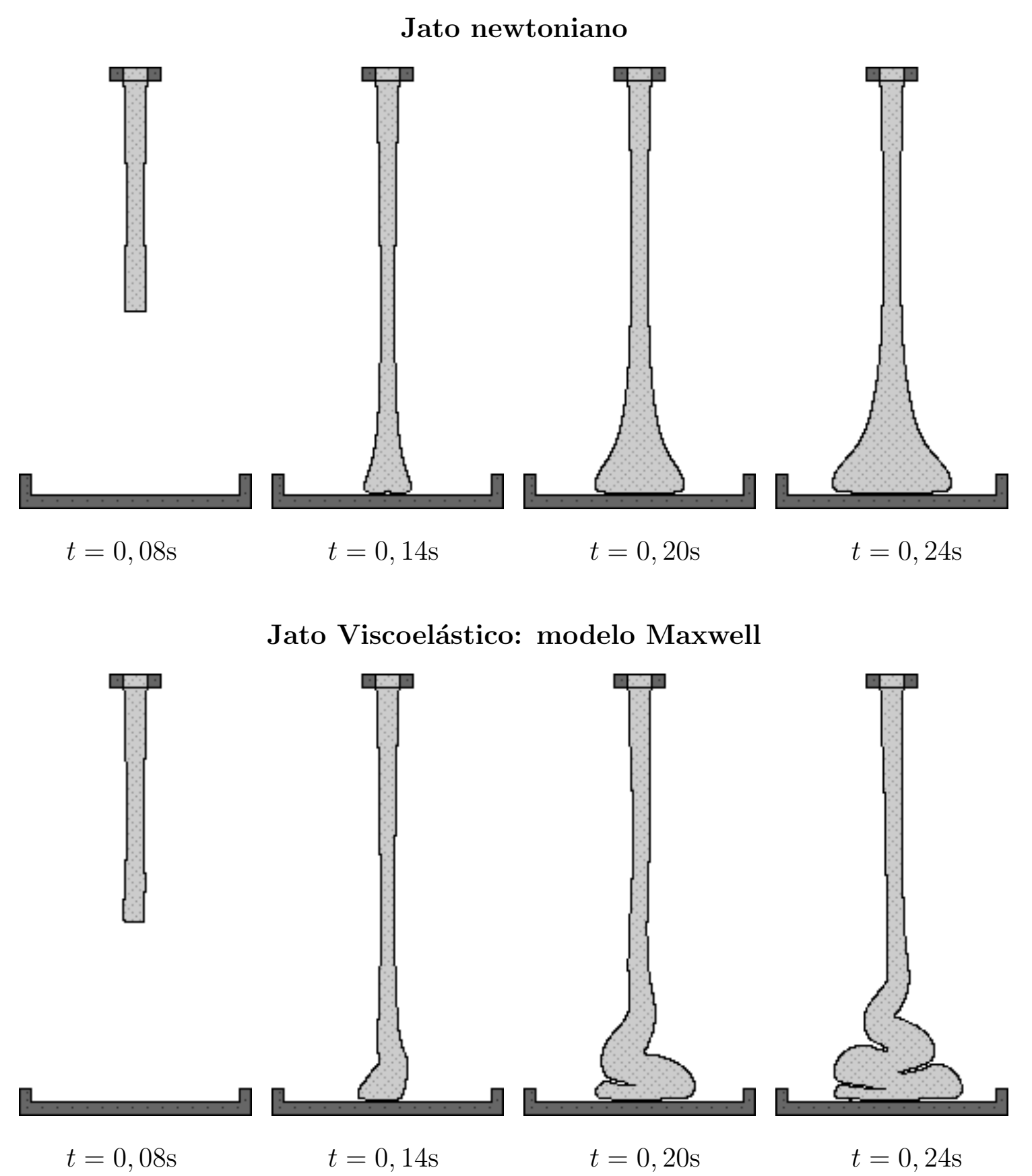

Figura 4.32: Simulação numérica do jato oscilante em diferentes tempos. Na parte superior da figura é apresentada a visualização para o modelo newtoniano com $R e=0,6$. $\mathrm{Na}$ parte inferior temos a visualização utilizando o modelo Maxwell com $R e=0,6$ e $W e=0,3$.

\subsubsection{Efeito da viscoelasticidade}

Com o objetivo de investigar o efeito da viscoelasticidade no problema do jato oscilante, realizamos várias simulações onde mantivemos o número de Reynolds fixo e aumentamos 


\section{Jato newtoniano}

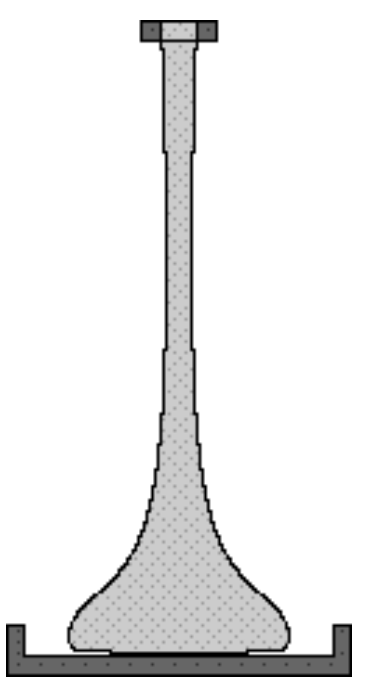

$t=0,28 \mathrm{~s}$

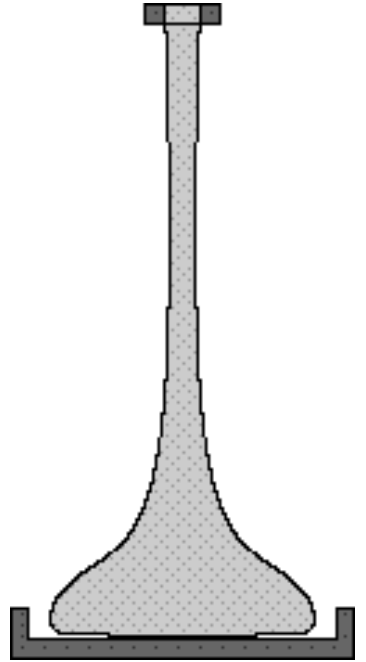

$t=0,32 \mathrm{~s}$

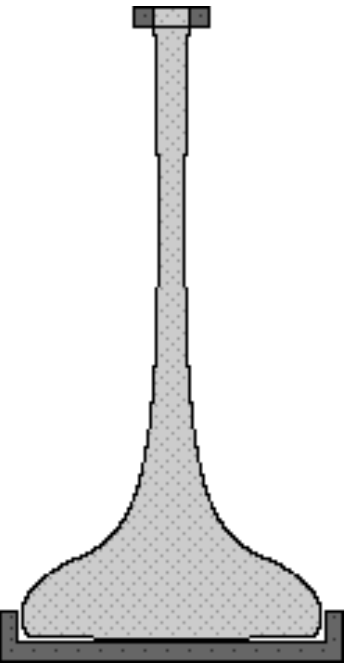

$t=0,36 \mathrm{~s}$

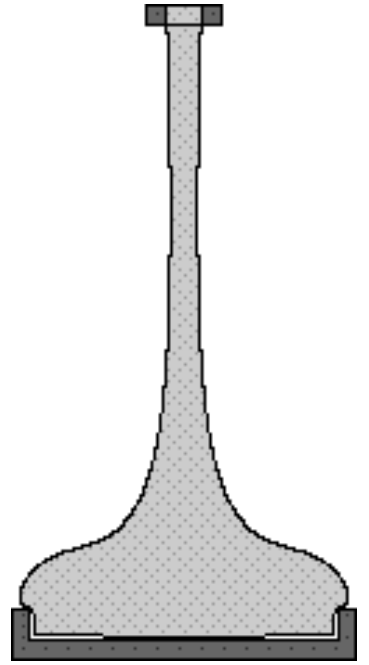

$t=0,40 \mathrm{~s}$

Jato Viscoelástico: modelo Maxwell

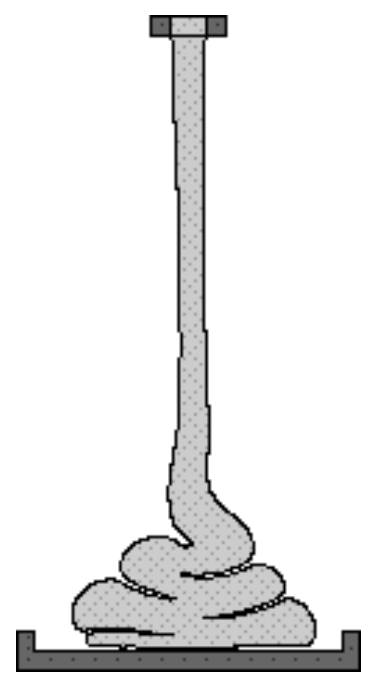

$t=0,28 \mathrm{~s}$

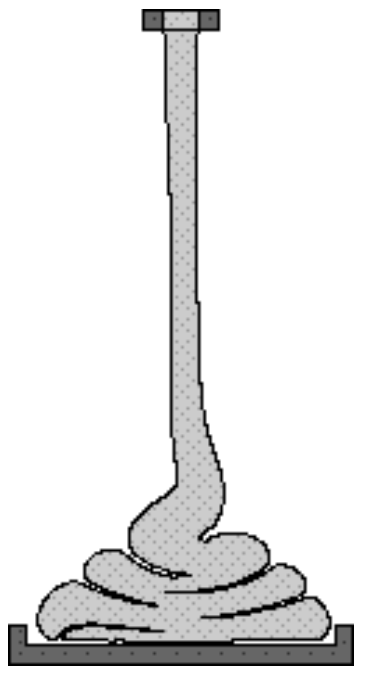

$t=0,32 \mathrm{~s}$

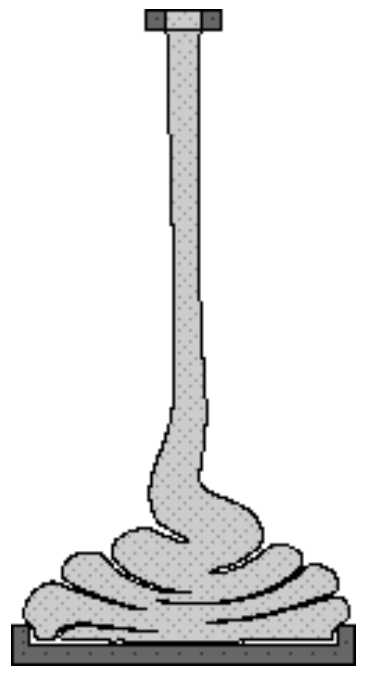

$t=0,36 \mathrm{~s}$

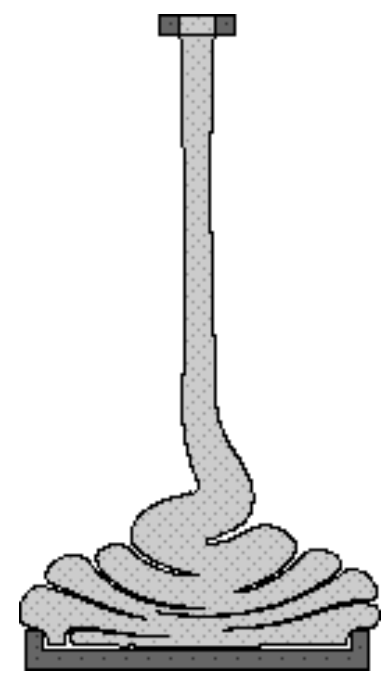

$t=0,40 \mathrm{~s}$

Figura 4.32. Continuação.

o número de Weissenberg. Utilizamos os dados acima com a mesma geometria, a mesma malha e as mesmas constantes $g, E P S$ e $F A C T$. Os parâmetros do fluido e a velocidade no injetor foram ajustados de modo a obtermos o número de Reynolds $R e=0,4$ e os números de Weissenberg $W e=0,1, W e=0,4$ e $W e=0,7$. Dessa maneira, utilizamos $U=0,4 \mathrm{~ms}^{-1}, \rho=1000 \mathrm{kgm}^{-3}, \eta_{0}=6$ Pa.s e as constantes do modelo de Maxwell $a$ e $\lambda$ 
foram:

$$
\begin{aligned}
& W e=0,1 \Longrightarrow a=4000,0 \mathrm{~Pa} \quad \text { e } \quad \lambda=0,0015 \mathrm{~s} \\
& W e=0,4 \Longrightarrow a=1000,0 \mathrm{~Pa} \quad \text { e } \quad \lambda=0,0060 \mathrm{~s} \\
& W e=0,7 \Longrightarrow a=571,49 \mathrm{~Pa} \quad \text { e } \quad \lambda=0,0105 \mathrm{~s}
\end{aligned}
$$

No total foram, realizadas três simulações e os resultados dessas simulações estão mostrados na figura 4.33. Como podemos observar, o jato ao atingir a superfície rígida apresentou o efeito do jato oscilante para todos os números de Weissenberg utilizados. Também podemos observar que antes do jato atingir a superfície rígida, a diferença entre as simulações não difere muito uma da outra e podemos dizer que com o aumento do número de Weissenberg diminui o tempo necessário para o jato alcançar a superfície rígida, o que sugere que a viscosidade do jato em queda livre diminui com o aumento do número de Weissenberg. Porém, após o jato atingir a superfície rígida, a diferença entre as simulações é mais notável. Os jatos com os números de Weissenberg $W e=0,1$ e $W e=0,4$ apresentaram o fenômeno do jato oscilante imediatamente após alcançar a superfície rígida com as oscilações ocorrendo próximas à superfície rígida. No modelo com o número de Weissenberg $W e=0,7$ ocorreram oscilações ao longo de todo o jato e ocorreu a propagação de uma onda que viaja em direção contrária ao escoamento até atingir o injetor (ver figura 4.34). Nos tempos $t=0,22 \mathrm{~s}$ e $0,24 \mathrm{~s}$ a onda está retornando na direção do escoamento o que faz com que as oscilações se aproximem da superfície rígida (ver figura 4.35). Esse efeito foi também observado por Tomé et al. [60] na simulação do jato oscilante de um fluido Oldroyd-B.

\subsubsection{Simulação do jato oscilante - modelo K-BKZ}

Para demonstrar que o método numérico desenvolvido nesse trabalho pode simular escoamentos com superfícies livres utilizando a equação constitutiva K-BKZ, apresentamos uma simulação do jato oscilante utilizando os mesmos parâmetros do fluido estudado por Quinzani et al. [52] (ver tabela 4.1). Os seguintes dados foram utilizados:

- Domínio computacional: $6,6 \mathrm{~cm} \times 10,6 \mathrm{~cm}$

- Malha utilizada: $\delta x=\delta y=1 \mathrm{~mm}(66 \times 106)$-células

- Aceleração gravitacional: $g=9,81 \mathrm{~ms}^{-2}$

- Altura do injetor à superfície rígida: $H=10 \mathrm{~cm}$ 


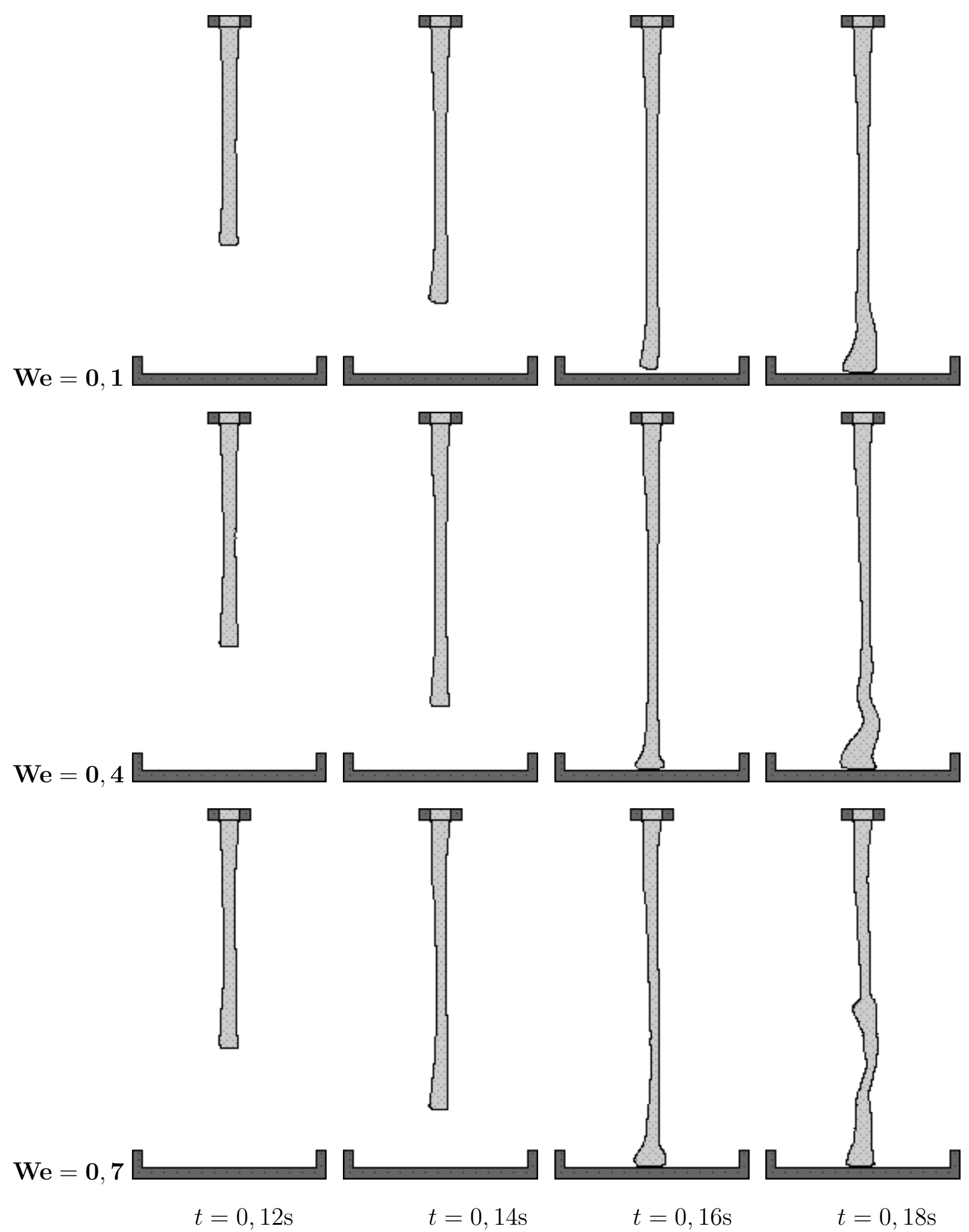

Figura 4.33: Simulação numérica do jato oscilante em diferentes tempos - Visualização do fluido $-R e=0,4$.

- Largura do injetor: $L=2 \mathrm{~cm}$

- Velocidade do jato no injetor: $U=0,1 \mathrm{~ms}^{-1}$ 
- Precisão da equação de Poisson: $E P S=10^{-7}$

- Fator para o cálculo do tamanho do passo no tempo: $F A C T=0,1$

- Definição do fluido:

- Viscosidade a baixas taxas de cisalhamento: $\eta_{0}=1,424 \mathrm{~Pa} . \mathrm{s}$

- Massa específica do fluido: $\rho=791,0 \mathrm{kgm}^{-3}$ (valor ajustado de modo que $\left.\nu=\eta_{0} / \rho=0,0018 \mathrm{~m}^{2} \mathrm{~s}\right)$

- Modelo de K-BKZ: $\alpha=10,0, \quad \beta=0,7$ e $\lambda_{\text {ref }}=0,06 \mathrm{~s}$

\begin{tabular}{cccc}
\hline \hline$k$ & $\lambda_{k}(\mathrm{~s})$ & $\eta_{k}(\mathrm{~Pa} \mathrm{~s})$ & $a_{k}(\mathrm{~Pa})$ \\
\hline 1 & 0,6855 & 0,040 & 0,058352 \\
2 & 0,1396 & 0,2324 & 1,664756 \\
3 & 0,0389 & 0,5664 & 14,560411 \\
4 & 0,0059 & 0.585 & 99,152542 \\
\hline
\end{tabular}

Logo, temos $R e=\rho U L / \eta_{0}=1,11$ e $W e=\lambda_{\text {ref }} U / L=0,3$. O código FreeFlow-2D simulou esse problema e a figura 4.36 apresenta a visualização do fluido em diferentes tempos. Como podemos observar, embora as condições de Cruickshank para um jato newtoniano não estejam satisfeitas, o jato modelado pela equação K-BKZ apresentou o fenômeno do jato oscilante. 

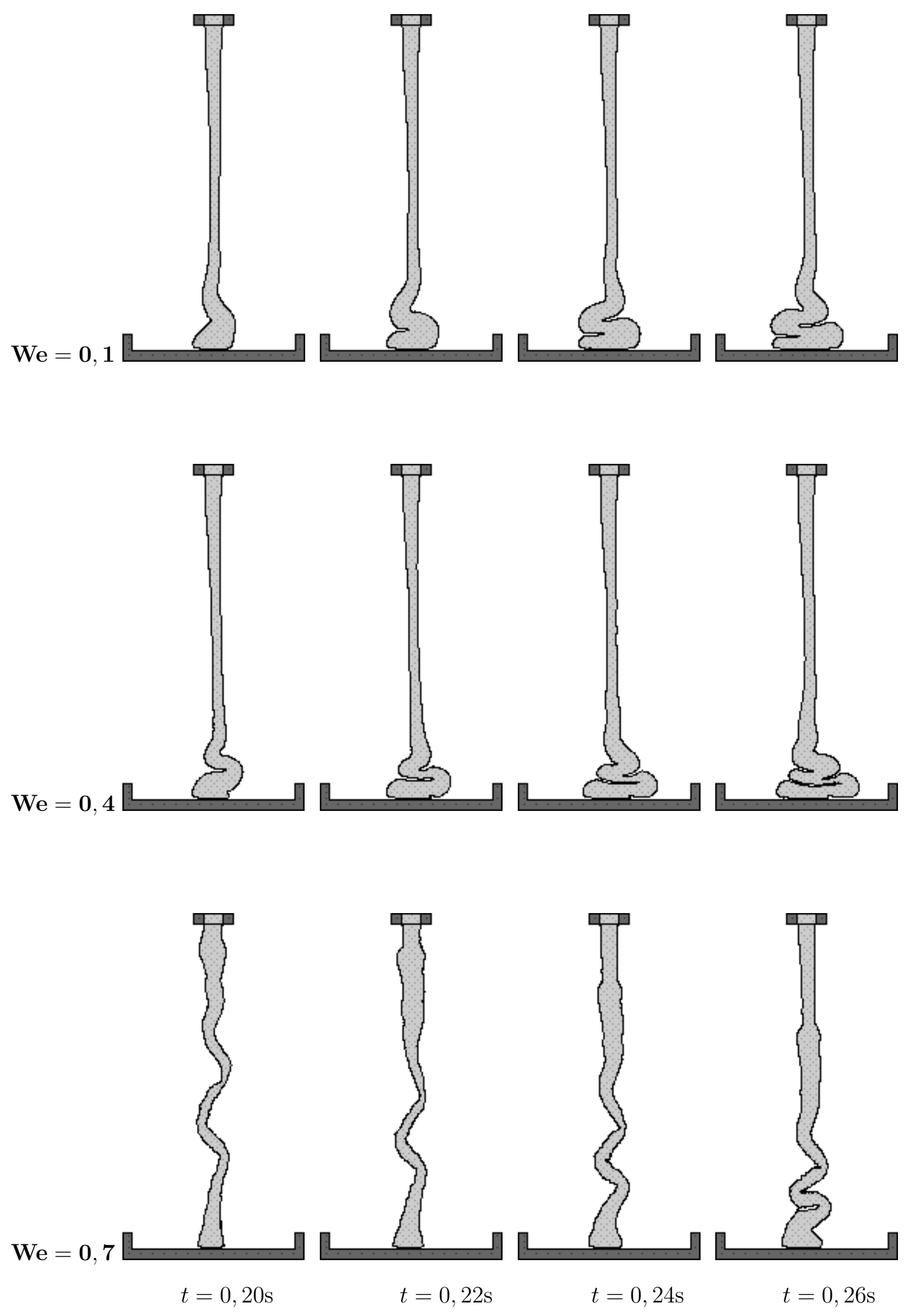

Figura 4.33. Continuação. 

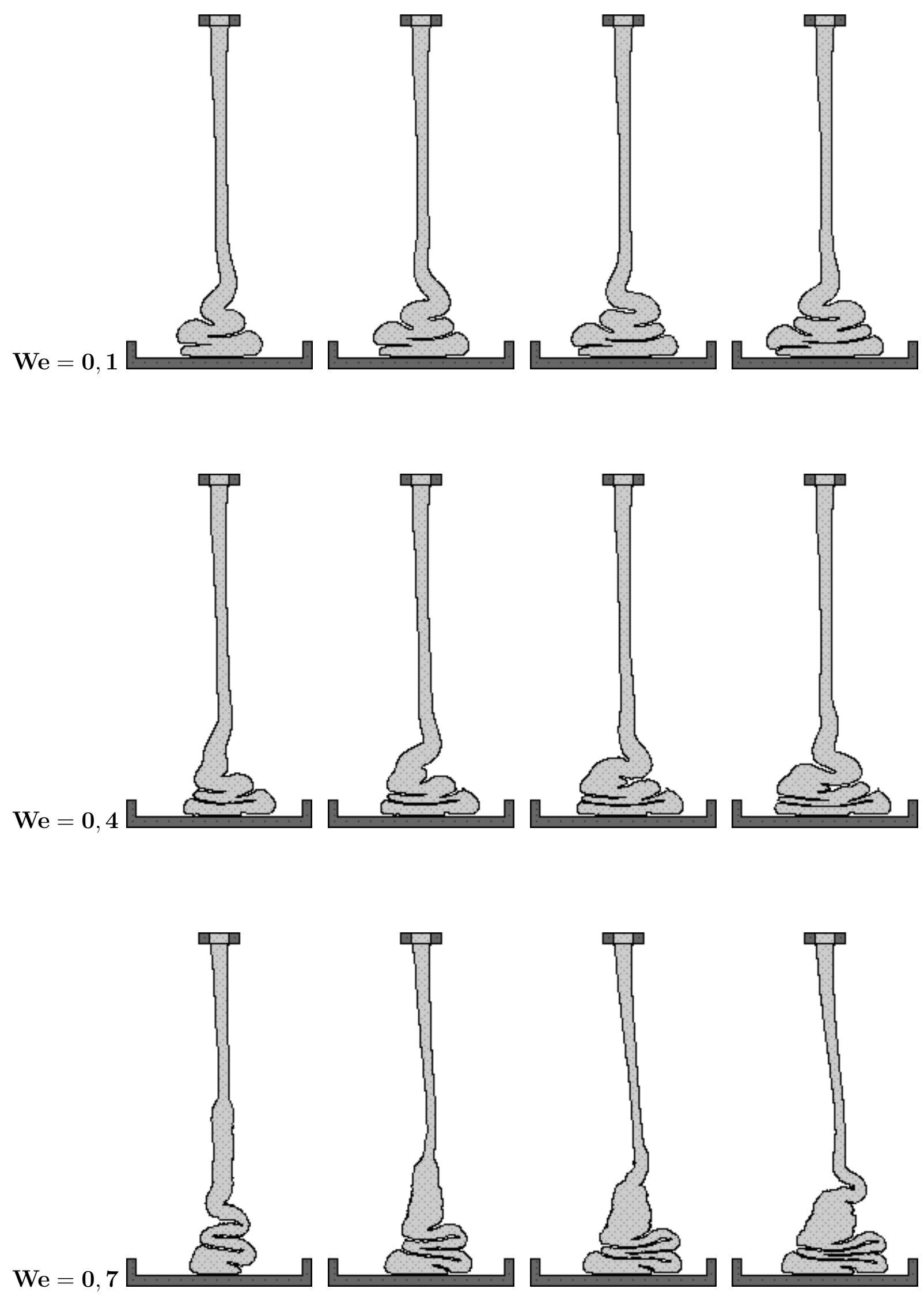

$$
t=0,28 \mathrm{~s}
$$$$
t=0,30 \mathrm{~s}
$$$$
t=0,32 \mathrm{~s}
$$$$
t=0,34 \mathrm{~s}
$$

Figura 4.33. Continuação. 

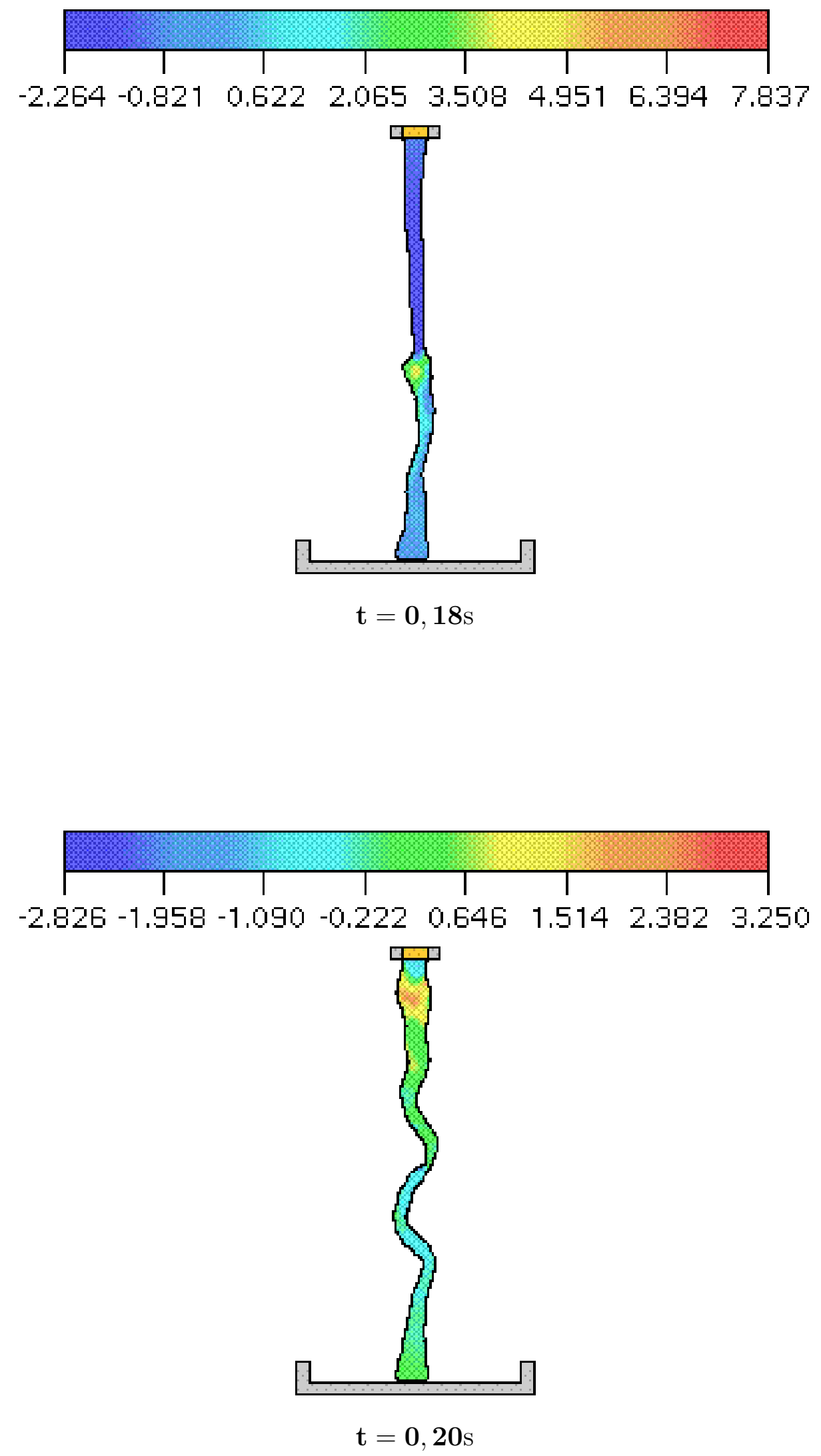

Figura 4.34: Simulação numérica do jato oscilante em diferentes tempos: $t=0,18 \mathrm{~s}$ e $t=0,20 \mathrm{~s} . R e=0,4$ e $W e=0,7$ - Visualização da componente $v$ da velocidade. 

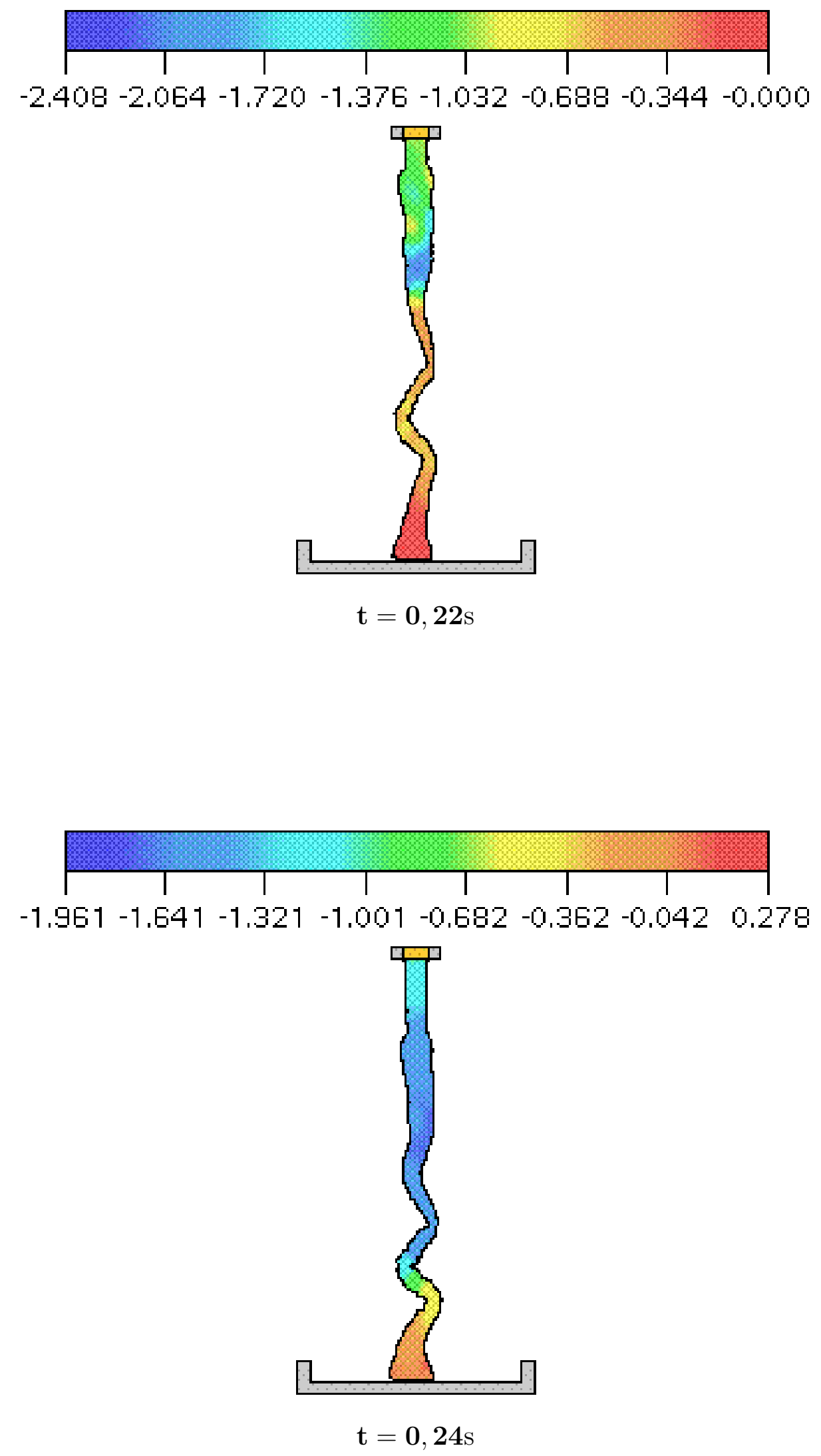

Figura 4.35: Simulação numérica do jato oscilante em diferentes tempos: $t=0,22 \mathrm{~s}$ e $t=0,24 \mathrm{~s} . R e=0,4$ e $W e=0,7$ - Visualização da componente $v$ da velocidade. 

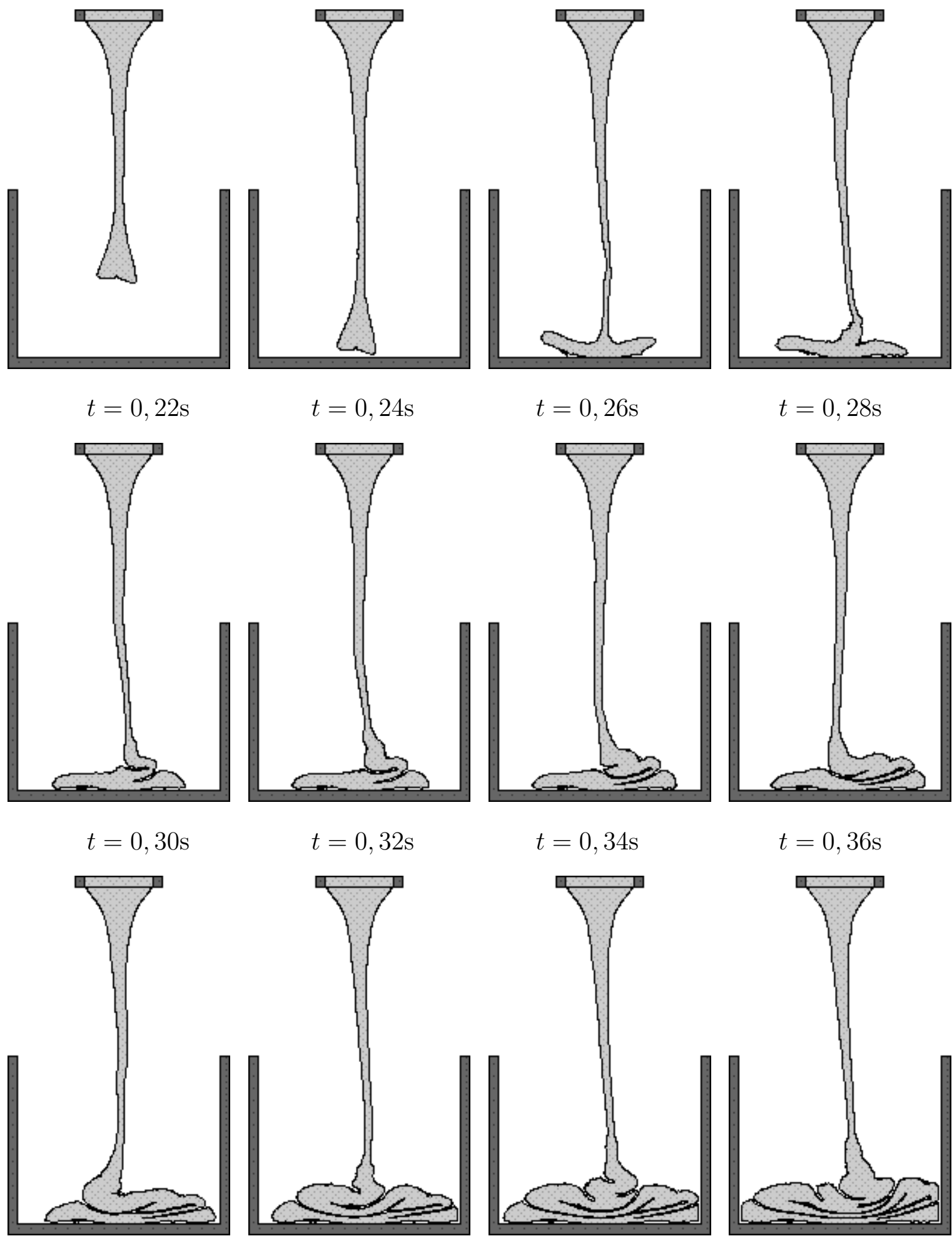

$$
t=0,40 \mathrm{~s}
$$$$
t=0,44 \mathrm{~s}
$$

$t=0,48 \mathrm{~s}$

$t=0,36 \mathrm{~s}$

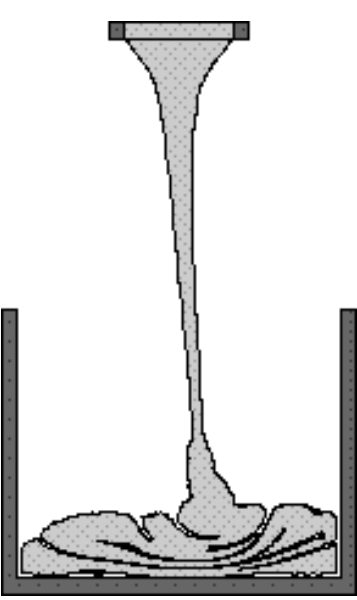

$$
t=0,52 \mathrm{~s}
$$

Figura 4.36: Modelo K-BKZ: Simulação numérica do jato oscilante em diferentes tempos - Visualização do fluido - $R e=1,11$ e $W e=0,3$. 


\section{Conclusão}

Nesta tese foi apresentada uma técnica numérica para simular escoamentos de fluidos viscoelásticos, governados pelas equações constitutivas integrais Maxwell e K-BKZ. O método numérico consiste na extensão do método GENSMAC para simular escoamentos incompressíveis governados pelos modelos constitutivos integrais Maxwell e K-BKZ e utiliza método de diferenças finitas em uma malha deslocada.

O tensor de Finger foi determinado com uma modificação do método de campos de deformação de Peters et al. [49], em que foram usadas técnicas diferentes para fazer a discretização do tempo passado, utilização do método de diferenças finitas para o cálculo do tensor de Finger e aplicação de um método de segunda ordem para o cálculo das tensões. Esse procedimento permitiu uma melhoria considerável para fluxos cisalhantes, nos quais o erro cometido está associado com o método de Euler usado para a aproximação do tensor de Finger. Neste caso, o número de campos utilizado (relacionado com o número $\mathrm{N}$ de subintervalos em $[0, t])$, tem pouca influência na precisão do método. Para escoamentos elongacionais uniaxiais constatou-se que o método desenvolvido nesta tese pode apresentar resultados não muito precisos devido à aproximação da componente do tensor de Finger, que neste caso é uma exponencial, por um polinômio de segunda ordem. O aumento do comprimento dos intervalos de integração contribui para a perda de precisão neste caso. Observou-se ainda que este problema pode ser atenuado se utilizarmos uma discretização uniforme do tempo passado. Entretanto, para tempos grandes pode haver problemas, já que os intervalos de integração inevitavelmente crescem com o tempo independentemente da forma como é feita a discretização. Portanto deve-se tomar cuidado ao simular processos que necessitam de tempos muito grandes.

A aplicação do método GENSMAC juntamente com a metodologia de cálculo do tensor de Finger possibilitou a simulação de escoamentos de fluidos viscoelásticos, utilizando 
modelos integrais no ambiente de simulação FreeFlow-2D. A validação do código foi feita através do escoamento entre placas paralelas, na qual a solução numérica para a velocidade e para as componentes de tensão obtidas com o modelo de Maxwell foram comparadas com as respectivas soluções analíticas no estado estacionário, mostrando excelente concordância. Além disso, a simulação deste mesmo problema com o modelo K-BKZ em diferentes malhas também mostrou a convergência do método à medida que a malha é refinada. E por fim, o código foi utilizado para simular o problema da contração planar 4:1, no qual os resultados foram comparados com os dados experimentais de Quinzani et al. [52]. Os resultados, apresentados na forma dimensional, foram considerados satisfatórios, pois conseguiram se aproximar qualitativamente bem dos dados experimentais. Em alguns casos, os resultados quantitativos também foram bons. A comparação com os dados numéricos de Azaiez et al. [5] mostraram semelhanças em situações nas quais não houve concordância com os dados experimentais, como é o caso do perfil de velocidade no eixo de simetria para $\mathrm{Re}=0,56$ e $\mathrm{We}=2,9$, nos quais os resultados numéricos obtidos com o código FreeFlow-2D e os resultados numéricos de Azaiez et al. mostraram um salto que não aparece nos dados experimentais.

Ainda com relação à simulação da contração planar 4:1 foram investigados o comportamento dos vórtices de canto e de 'lip vortices' para o modelo Maxwell, onde o número de Reynolds foi mantido fixo e igual a $R e=1$ e $R e=0,1$ e o número de Weissenberg foi variado. Nesta situação, observou-se o decrescimento do tamanho do vórtice de canto com o aumento do número de Weissenberg. Este comportamento está em concordância com outros trabalhos da literatura. Entretanto, outras investigações devem ser feitas de modo a obter mais resultados e mais comparações com dados da literatura, especialmente com relação ao aparecimento ou não de 'lip vortices' a partir de um certo número de Weissenberg. Ainda não está claro se o aparecimento dos 'lip vortices' é conseqüência do modelo integral utilizado ou da influência do método numérico utilizado, já que ao se fazer um refinamento da malha para o caso de $W e=4$, observou-se que o tamanho do 'lip vortex' é menor do que o tamanho obtido na malha mais grossa. Investigações com relação ao refinamento da malha são portanto necessárias. É preciso trabalhar em um processo de otimização do código, de modo a tornar as simulações mais rápidas.

Nas simulações de escoamentos com superfícies livres utilizamos o método numérico para simular o problema do jato oscilante. Foram realizadas várias simulações e os re- 
sultados obtidos mostraram que o número de Weissenberg tem bastante influência no fenômeno do jato oscilante. Utilizando os dados de um fluido real, utilizamos o modelo K-BKZ para simular um jato incidindo em uma superfície plana e obteve-se o fenômeno do jato oscilante. Em todas as simulações realizadas, as condições para jatos newtonianos de Cruickshank [13] não foram satisfeitas e portanto acreditamos que os resultados obtidos com o modelo de Maxwell e K-BKZ foram devidos à viscoelasticidade do fluido.

Além do que já foi dito anteriormente, outro projeto para desenvolvimento é a aplicação da técnica numérica apresentada nesta tese na simulação de escoamentos axisimétricos de fluidos viscoelásticos, utilizando os modelos integrais aqui apresentados. A extensão para o caso tridimensional geral também é possível, entretanto poderia ser muito caro do ponto de vista computacional, uma vez que a quantidade de memória necessária para guardar as informações da história de deformação pode vir a ser proibitiva. Nesta linha deve-se primeiro investigar técnicas de armazenamento desta informação sem a necessidade de guardar a história de deformação em todas as células. 


\section{Referências Bibliográficas}

[1] M. A. Alves, P. J. Oliveira, And F. T. Pinho, Benchmark solutions for the flow of oldroyd-B and PTT fluids in planar contractions, J. Non-Newtonian Fluid Mech., 110 (2003), pp. 45-75.

$[2]$ _ A convergent and universally bounded interpolation scheme for treatment of advection, Int. Jour. Num. Meth. Fluids, 41 (2003), pp. 47-75.

[3] M. A. Alves, F. T. Pinho, And P. J. Oliveira, Visualization of boger fluid flows in a 4:1 square-square contraction, AIChE Journal, 51 (2005), pp. 2908-2922.

[4] A. A. Amsden And F. H. Harlow, A simplified mac technique for incompressible fluid flow calculation, J. Comp. Phys., 6 (1970), pp. 322-325.

[5] J. Azaiez, R. GuÉnette, And A. AÏT-Kadi, Numerical simulation of viscoelastic flows through a planar contraction, J. Non-Newtonian Fluid Mech., 62 (1996), pp. $253-277$.

[6] G. K. Batchelor, An Introduction to Fluid Dynamics, Cambridge, 1967.

[7] B. Bernstein, E. A. Kearsley, And L. J. Zapas, A study of stress relaxation with finite strain, Transactions of Society of Rheology, VII (1963), pp. 391-410.

[8] R. B. Bird, R. C. Armstrong, And O. Hassager, Dynamics of Polymeric Liquids, vol. 1, Jhon Wiley \& Sons, 1987.

[9] D. Boger, D. Hur, And R. Binnington, Further observations of elastic effects in tubular entry flows, J. Non-Newtonian Fluid Mech., 20 (1986), pp. 31-49.

[10] R. E. Bretas And M. A. D’Avila, Reologia de Polímeros Fundidos, Editora UFSCar, 2000. 
[11] A. Castello, M. F. Tomé, C. N. L. César, S. Mckee, and J. A. Cuminato, Freeflow: an integrated simulation system for three dimensional free surface flows, Computing and Visualization in Science, 2 (2000), pp. 199-210.

[12] A. J. Chorin And J. E. Marsden, A Mathematical Introduction to Fluid Mechanics, Springer-Verlag, 1992.

[13] J. O. CRuiCKSHANK, Low-reynolds-number instabilities in stagnating jet flows, Journal of Fluid Mechanics, 193 (1988), pp. 111-127.

[14] J. O. Cruickshank And B. R. Munson, Viscous-fluid buckling of plane axisymmetric jets, Journal of Fluid Mechanics, 113 (1981), pp. 221-239.

[15] D. M. De Carvalho, M. F. Tomé, J. A. Cuminato, A. Castelo, and V. G. FERREIRA, A numerical technique for solving the Maxwell model for free surface flows, TEMA - Tendências em Matemática Aplicada e Computacional, 5 (2004), pp. $185-204$.

[16] R. Evans AND K. WALters, Flow characteristics associated with abrupt changes in geometry in the case of highly elastic liquids, J. Non-Newt. Fluid Mech., 20 (1986), pp. 11-29.

[17] _ Further remarks on the lip-vortex mechanism of vortex enhancement in planarcontraction flows, J. Non-Newt. Fluid Mech., 32 (1989), pp. 95-105.

[18] V. G. Ferreira, M. F. Tomé, N. Mangiavacchi, A. Castelo, J. A. Cuminato, A. Fortuna, And S. MCKeE, High-order upwinding and the hydraulic jump, Int. J. Numer. Meth. Fluids, 39 (2002), pp. 549-583.

[19] L. Grossi, Desenvolvimento de métodos numéricos para a simulação de escoamentos não-newtonianos e viscoelásticos com superfícies livres, $\mathrm{PhD}$ thesis, ICMC-USP - São Carlos - Brasil, 2003.

[20] R. GuÉnette And M. Fortin, A new mixed finite element method for computing viscoelastic flows, J. Non-Newt. Fluid Mech., 60 (1995), pp. 27-52.

[21] F. Harlow and J. E. WelCh, Numerical calculation of time-dependent viscous incompressible flow of fluid with a free surface, Phys. Fluids, 8 (1965), pp. 2182-2189. 
[22] M. A. Hulsen, E. A. J. F. Peters, and B. H. A. A. van den Brule, A new approach to the deformation fields method for solving complex flows using integral constitutive equations, J. Non-Newt. Fluid Mech., 98 (2001), pp. 201-221.

[23] E. IsaAcson And H. B. Keller, Analysis of numerical methods, Willey, 1996.

[24] A. KAYE, Non-newtonian flow in incompressible fluids, College of Aeronautics, (1962).

[25] H. C. Kuhlmann and H.-J. Rath, Free Surface Flows, SpringerWienNewYork, 1998.

[26] R. Larson, Constitutive Equations for Polymer Melts and Solutions, ButterworthHeinemann, 1988.

[27] — , The Structure and Rheology of Complex Fluids, Oxford University Press, 1999.

[28] J. L.Doricio, M. F. Tomé, A. Castelo, J. A. Cuminato, and V. G. FerREIRA, Numerical simulation of viscoelastic free surface flows using the second order constitutive equation, in Proceedings of COBEM2005, 2005.

[29] X. L. LuO, A control volume approach for integral viscoelastic models and its application to contraction flow of polymer melts, J. Non-Newtonian Fluid Mech., 64 (1996), pp. 173-189.

[30] X. L. LuO And E. Mitsoulis, An efficient algorithm for strain history tracking in finite element computations of non-newtonian fluids with integral constitutive equations, Int. J. Num. Meth. Fluids, 11 (1990), pp. 1015-1031.

[31] — A numerical study of the effect of elongational viscosity on vortex growth in contraction flows of polyethylene, J. Rheol., 34 (1990), pp. 309-342.

[32] X. L. Luo AND R. I. TANnER, A streamline element scheme for solving viscoelastic flow problems Part I : Diffential constitutive equations, J. Non-Newtonian Fluid Mech., 21 (1986), pp. 179-199.

[33] — - A streamline element scheme for solving viscoelastic flow problems Part II : Integral constitutive models, J. Non-Newtonian Fluid Mech., 22 (1986), pp. 61-89. 
[34] C. W. Macosko, Rheology: principles, measurements and applications, VHC, 1994.

[35] E. Mitsoulis, Numerical simulation of planar entry flow for a polyisobutylene solution using an integral constitutive equation, J. Rheol., 37 (1993), pp. 1029-1040.

[36] _ - Numerical simulation of entry flow of fluid S1, J. Non-Newtonian Fluid Mech., 78 (1998), pp. 187-201.

[37] _ Further results for the entry flow of fluid S1, J. Non-Newtonian Fluid Mech., 97 (2001), pp. 149-158.

[38] —_, Numerical simulation of entry flow of the IUPAC-LPDE melt, J. NonNewtonian Fluid Mech., 97 (2001), pp. 13-30.

[39] E. Mitsoulis, M. Schwetz, And H. Münstedt, Entry flow of LPDE melts in a planar contraction, J. Non-Newtonian Fluid Mech., 111 (2003), pp. 41-61.

[40] G. Mompean and M. Deville, Unsteady finite volume simulation of Oldroyd-B fluid through a three-dimensional planar contraction, J. Non-Newtonian Fluid Mech., 72 (1997), pp. 253-279.

[41] C. M. Oıshi, Análise e implementação de métodos implícitos no sistema FreeFlow2d, master's thesis, ICMC-USP, 2004.

[42] J. Oliveira, Desenvolvimento de um sistema de simulação de escoamentos de fluidos com superfícies livres bidimensionais, master's thesis, ICMC-USP, 1999.

[43] P. Olley, An adaptation of the separable $K-B K Z$ equation for comparable response in planar and axisymmetric flow, J. Non-Newtonian Fluid Mech., 95 (2000), pp. 3553.

[44] P. Olley and P. D. Coates, An approximation to the $K-B K Z$ constitutive equation, J. Non-Newtonian Fluid Mech., 69 (1997), pp. 239-254.

[45] P. Olley, R. Spares, And P. D. Coates, A method for implementing timeintegral constitutive equations in commercial CFD packages, J. Non-Newtonian Fluid Mech., 86 (1999), pp. 337-357. 
[46] R. G. Owens And T. N. Phillips, Computational Rheology, Imperial College Press, 2002.

[47] A. C. Papanastasiou, L. Scriven, and C. Macosko, An integral constitutive equation for mixed flows: Viscoelastic characterization, Journal of Rheology, 27 (1983), pp. 387-410.

[48] E. A. J. F. Peters, Polymers in flow : modelling and simulation, PhD thesis, Technische Universiteit Delft, 2000.

[49] E. A. J. F. Peters, M. A. Hulsen, and B. H. A. A. van den Brule, Instationary Eulerian viscoelastic flow simulation using time separable Rivlin-Sawyers constitutive equations, J. Non-Newtonian Fluid Mech., 89 (2000), pp. 209-228.

[50] T. Phillips AND A. Williams, Viscoelastic flow through a planar contraction using a semi-lagrangian finite volume method, Journal of Non-Newtonian Fluid Mechanics, 87 (1999), pp. 215-246.

[51] B. Purnode And M. Crochet, Flows of polymer solutions through contractions part 1: flows of polyacrylamide solutions through planar contractions, J. NonNewtonian Fluid Mech., 65 (1996), pp. 269-289.

[52] L. M. Quinzani, R. C. Armstrong, And R. A. Brown, Birefringence and laserDoppler velocimetry (LDV) studies of viscoelastic flow through a planar contraction, Journal of Non-Newtonian Fluid Mechanics, 52 (1994), pp. 1-36.

[53] L. M. Quinzani, G. H. McKinley, R. A. Brown, and R. C. Armstrong, Modeling the rheology of polyisobutylene solutions, J. Rheol., 34 (1990), pp. 705-748.

[54] H. K. RASmussen, Lagrangian viscoelastic flow computations using the RivlinSawyers constitutive model, J. Non-Newtonian Fluid Mech., 92 (2000), pp. 227-243.

[55] R. S. Rivlin and K. N. Sawyers, Ann. Rev. Fluid Mech., 3 (1971), p. 117.

[56] R. I. TAnner, Engineering Rheology, Clarendon Press - Oxford, 1988.

[57] M. F. Tomé, A. Castelo, J. A. Cuminato, N. Mangiavacchi, and S. McKEE, GENSMAC3D: A numerical method for solving three-dimensional free surface flows, Intern. J. Numer. Meth. Fluids, 37 (2001), pp. 747-796. 
[58] M. F. Tomé, A. Castelo, J. A. Cuminato, J. Murakami, R. Minghim, C. F. OliveIRA, AND S. MCKeE, Numerical simulation of axisymmetric free surface flows, J. Comput. Phys., 157 (2000), pp. 441-472.

[59] M. F. Tomé, B. Duffy, And S. McKeE, A numerical technique for solving unsteady non-Newtonian free surface flows, J. non-Newt. Fluid Mech., 62 (1996), pp. 9-34.

[60] M. F. Tomé, N. Mangiavacchi, J. A. Cuminato, A. Castelo, and S. McKEE, A finite difference technique for simulating unsteady viscoeslastic free surface flows, J. non-Newt. Fluid Mech., 106 (2002), pp. 61-106.

[61] M. F. Tomé And S. MCKeE, GENSMAC : A computational marker and cell method for free surface flows in general domains, J. Comput. Phys., 110 (1994), pp. 171-186.

[62] — Numerical simulation of viscous flow: Buckling of planar jets, Int. J. Numer. Meth. Fluids, 29 (1999), pp. 705-718.

[63] M. F. Tomé, S. McKee, L. Barratt, D. Jarvis, and A. Patrick, An experimental and numerical investigation of container filling with viscous liquids, Int. J. Numer. Meth. Fluids, 33 (1999), pp. 1333-1353.

[64] C. Truesdell, The Elements of Continuum Mechanics, Springer-Verlag, 1966.

[65] M. Viriyayuthakorn and B. Caswell, Finite element simulation of viscoelastic flow, J. Non-Newtonian Fluid Mech., 6 (1980), pp. 245-267.

[66] K. Walters And M. F. WeBster, The distinctive CFD challenges of computational rheology, Int. J. Numer. Meth. Fluids, 43 (2003), pp. 577-596.

[67] S. A. White And D. G. BAIRD, The importance of extensional flow properties on planar entry flow patterns of polymer melts, J. Non-Newtonian Fluid Mech., 20 (1986), pp. 93-101.

[68] —_ Flow visualization and birefringence studies on planar entry flow behavior of polymer melts, J. Non-Newtonian Fluid Mech., 29 (1988), pp. 245-267. 
[69] — Numerical simulation studies of the planar entry flow of polymer melts, J. Non-Newtonian Fluid Mech., 30 (1988), pp. 47-71.

[70] S. A. White, A. Gotsis, And D. G. Baird, Review of the entry flow problem: Experimental and numerical, J. Non-Newtonian Fluid Mech., 24 (1987), pp. 121-160.

[71] S. Xue, N. Phan-Thien, And R. I. Tanner, Numerical study of secundary flows of viscoelastic fluid in straight pipes by an implicit finite volume method, J. NonNewtonian Fluid Mech., 74 (1998), pp. 195-245. 